Florida International University FIU Digital Commons

FIU Electronic Theses and Dissertations

University Graduate School

3-30-2011

\title{
The Peopling of the Bahamas: A Phylogeographical Perspective
}

Tanya M. Simms

Florida International University, tsimm002@fiu.edu

DOI: $10.25148 /$ etd.FI1 1042605

Follow this and additional works at: https://digitalcommons.fiu.edu/etd

\section{Recommended Citation}

Simms, Tanya M., "The Peopling of the Bahamas: A Phylogeographical Perspective" (2011). FIU Electronic Theses and Dissertations. 408.

https://digitalcommons.fiu.edu/etd/408

This work is brought to you for free and open access by the University Graduate School at FIU Digital Commons. It has been accepted for inclusion in FIU Electronic Theses and Dissertations by an authorized administrator of FIU Digital Commons. For more information, please contact dcc@fiu.edu. 


\section{FLORIDA INTERNATIONAL UNIVERSITY}

Miami, Florida

THE PEOPLING OF THE BAHAMAS: A PHYLOGEOGRAPHICAL PERSPECTIVE

A dissertation submitted in partial fulfillment of the

requirements for the degree of

DOCTOR OF PHILOSOPHY

in

BIOLOGY

by

Tanya M. Simms

2011 
To: Dean Kenneth G. Furton

College of Arts and Sciences

This dissertation, written by Tanya M. Simms, and entitled The Peopling of the Bahamas: A Phylogeographical Perspective, having been approved in respect to style and intellectual content, is referred to you for judgment.

We have read this dissertation and recommend that it be approved.

Alejandro Barbieri

Bruce McCord

DeEtta Mills

George Duncan

Rene J. Herrera, Major Professor

Date of Defense: March 30, 2011

The dissertation of Tanya M. Simms is approved.

$\begin{array}{r}\hline \begin{array}{c}\text { Dean Kenneth G. Furton } \\ \text { College of Arts and Sciences }\end{array} \\ \hline \begin{array}{r}\text { Interim Dean Kevin O’Shea } \\ \text { University Graduate School }\end{array}\end{array}$

Florida International University, 2011 


\section{DEDICATION}

This dissertation is dedicated to my parents, Michael L. Simms and Sheila A. Simms, whose support, understanding and belief in me helped me successfully complete this project. 


\section{ACKNOWLEDGMENTS}

I gratefully acknowledge my mentor, Dr. Rene J. Herrera, and the members of my committee, Dr. Alejandro Barbieri, Dr. Bruce McCord, Dr. DeEtta Mills and Dr. George Duncan, for their support and guidance on this tumultuous journey. Special thanks are given to my collaborators at the Forensic Department of the Royal Bahamas Police Force (RBPF), including Chief Superintendent Quinn McCartney, Insp. Oswald Nixon, Cpl. Sheria King, Sgt. Melisande Bassett, Cpl. Shemieko Aurthur, Cpl. Raquel Francis, Cpl. Shanelle Sands, Cpl. Christina Thompson, Cpl. Thamera Campbell, Cpl. Cyprian Collie, Const. Charles Bonimy, Cpl. Aaron Wilson and Const. Elkia Coakley, for partaking in this endeavor with me. I am also grateful to Rick Lowe, Sammuel Sawyer and George Myers for their monetary donations which assisted with travel expenses incurred during the collection phase of the project. Members of my lab, including Annia Mesa, Carol Rodriguez, Carolina Chiou, Chantel Garcia, Daniel Puebla, Diane Rowold, Dianne Barrett, Emanuel Martinez, Evelyn Ramirez, Laura Hadden, Luis Rivera, Ketaki Deshpande, Kristian Herrera, Laura Hadden, Lisa Ibarra-Rivera, Marisil Wright, Maria Regueiro, Maria Christina Terreros, Michelle Hernandez, Omar Perez, Rob Lowery, Rosa Rodriguez, Sheyla Mirabal, Sylvia Calderon, Shilpa Chennakrishnaiah, Tenzin Gayden and Vivian Mendoza, also deserve special thanks for their technical support and/or constructive criticisms. Finally, I would like to recognize the University Graduate School of Florida International University for awarding me with the Dissertation Year Fellowship (Summer 2010) which allowed me to complete the writing portion of this project. 
ABSTRACT OF THE DISSERTATION

THE PEOPLING OF THE BAHAMAS: A PHYLOGEOGRAPHICAL PERSPECTIVE by

Tanya M. Simms

Florida International University, 2011

Miami, Florida

Professor Rene J. Herrera, Major Professor

During the past 500 years, the Bahamas has been influenced by a wide array of settlers, including but not limited to, the Arawak Indians, Eleutherian Adventurers, British Loyalists, Creole slaves, liberated Africans as well as Chinese, Greek, Jewish, Lebanese, Jamaican and Haitian migrants. To date, however, only a few reports analyzing the genetic makeup and population dynamics of the Bahamas have been published, making this work pivotal in the endeavor to ascertain the genetic ancestry of these groups. As such, the current investigation was undertaken to genetically characterize six of the more densely populated islands throughout the Northwest (Grand Bahama and Abaco) and Central (Eleuthera, Exuma, Long Island and New Providence) Bahamas using different forensic marker systems. When autosomal STR markers are employed, the Bahamian collections were all found to receive differential contributions from the African, European, East Asian and Native American collections utilized in the analyses. Similar findings were also observed for two other Afro-Caribbean populations, Haiti and Jamaica, although the latter populace was found to share a greater proportion of its autosomal component with non-African sources than the former. On the contrary, analysis of the six Bahamian collections using high-resolution Y-chromosome markers 
identifies genetic signals emanating exclusively from Africans and Europeans, but this is likely the result of smaller sample sizes collected from each island and/or sex-biased gene flow from East Asian and Native American groups. 


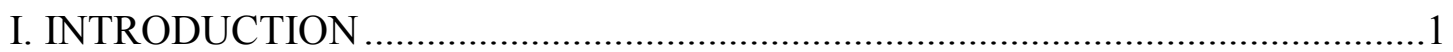

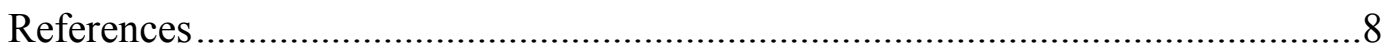

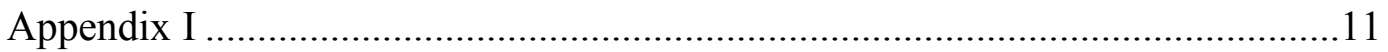

II. THE GENETIC LEGACY OF THE TRANSATLANTIC SLAVE TRADE IN THE ISLAND OF NEW PROVIDENCE.........................................................12

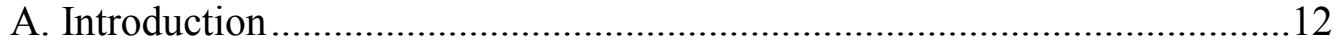

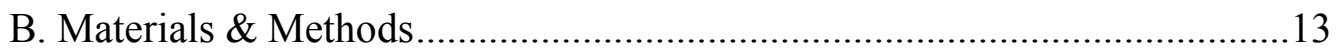

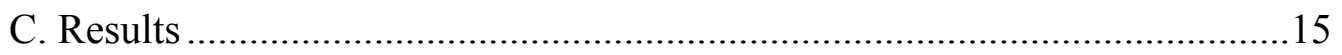

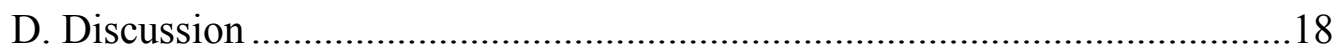

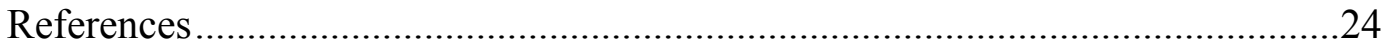

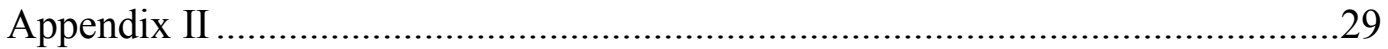

III. THE GENETIC STRUCTURE OF POPULATIONS FROM HAITI AND JAMAICA REFLECT DIVERGENT DEMOGRAPHIC HISTORIES .......................49

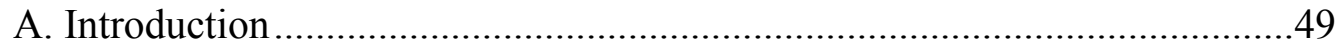

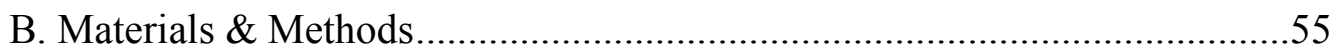

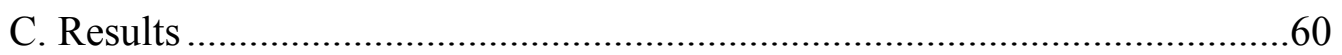

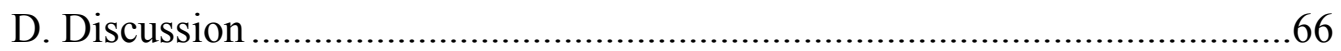

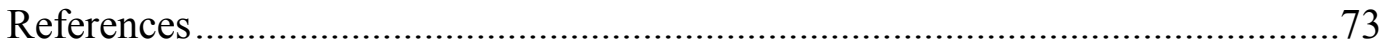

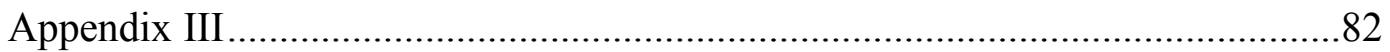

IV. DIVERGENT GENETIC STRATA IN FIVE BAHAMIAN ISLANDS .............104

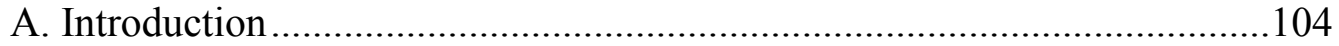

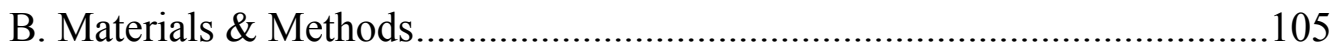

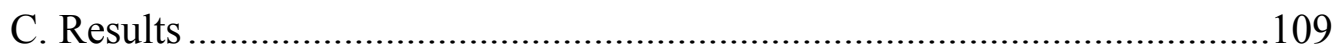

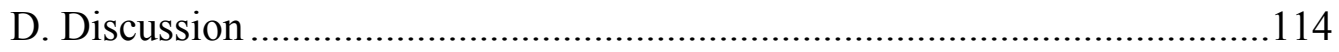

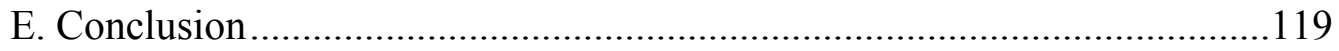

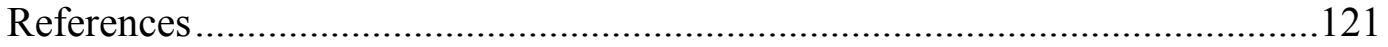

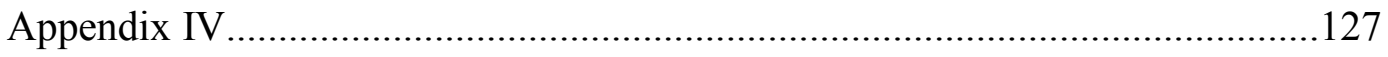

V. THE PATRIARCHAL GENETIC SUBSTRATA OF THE BAHAMIAN

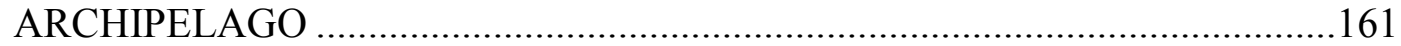

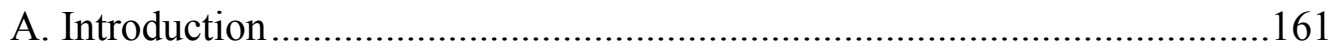

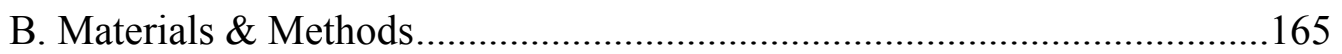

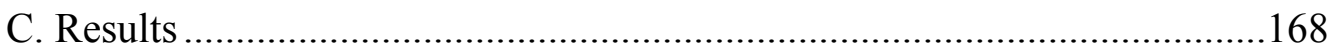

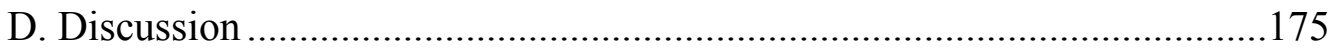




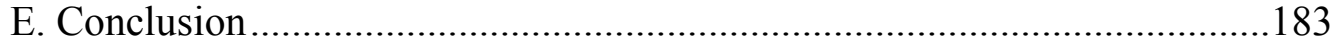

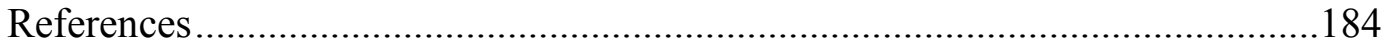

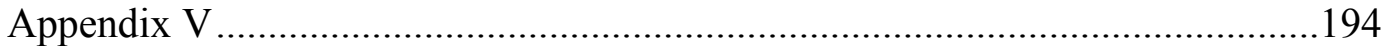

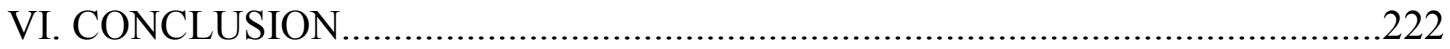

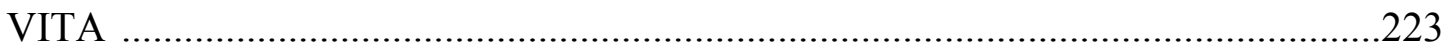




\section{LIST OF FIGURES}

FIGURE

PAGE

\section{Chapter I}

Fig. 1: Map of the Bahamas illustrating the islands comprising the northwest, central and southeast portions of the archipelago

\section{Chapter II}

Fig. 1A: Map of the Bahamian archipelago indicating the island of New Providence.

Fig. 1B: Map of Africa illustrating ports of departure (arrows) of African slaves during the Transatlantic Slave Trade and also the location of reference African collections used in the analyses

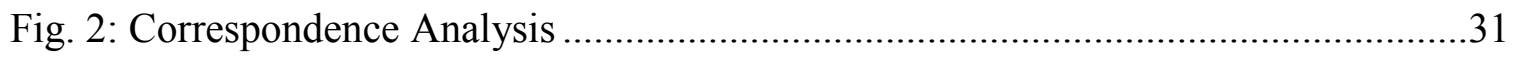

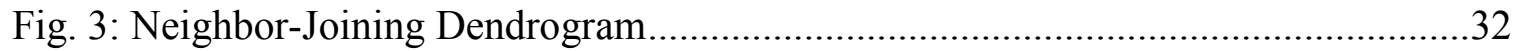

\section{Chapter III}

Fig. 1A: Multidimensional Scaling plot (Stress $=0.10202)$ of all populations at 15 loci.

Fig 1B: Multidimensional Scaling plot $($ Stress $=0.19149)$ of the African derived collections

Supporting Information Figure 1: Histograms comparing allele frequency data for Haiti and Jamaica per STR locus

Supporting Information Figure 2: Multidimensional Scaling Plot $($ Stress $=0.13124)$ of all populations at 13 loci

Supporting Information Figure 3: Structure bar plots assuming three and four ancestral populations $(\mathrm{K}=3$ and $\mathrm{K}=4)$ 


\section{Chapter IV}

Fig. 1: Geographic locations of Abaco (ABA), Eleuthera (ELU), Exuma (EXU), Grand Bahama (GRB) and Long Island (LBG) within the Bahamian archipelago

Fig. 2A: Multidimensional Scaling plot $($ Stress $=0.10286)$ of all populations

Fig. 2B: Multidimensional Scaling plot (Stress $=0.22533)$ of African and

African-derived collections.

Supplementary Figure 1: Histograms comparing allelic distributions in the five Bahamian populations surveyed

Supplementary Figure 2: Structure bar plots assuming three $(K=3)$ and four $(K=4)$ ancestral populations

\section{Chapter V}

Fig.1: Hierarchical phylogenetic relationships and frequencies (\%) of the 44 paternal haplogroups observed in the six Bahamian islands analyzed in the current investigation. M75, P147, P177, P123 and P143 (in italics) were not genotyped but were included for phylogenetic context

Fig. 2A: Global Y-chromosome frequency distribution at the level of the major haplogroups (A-T)

Fig. 2B: Frequency distributions of Y-chromosome E sub-haplogroups within the

Bahamian archipelago.

Fig. 3: Multidimensional Scaling plot based on Y-haplogroup frequency data of the populations listed in Table 1

Fig. 4A: Network projection based on the Y-STR profiles within sub-haplogroup E1b1a7a-U174

Fig. 4B: Network projection based on the Y-STR profiles within sub-haplogroup E1b1a8-U175

Fig. 5: Network projection based on the Y-STR profiles within sub-haplogroup R1b1b1-M269

Fig. 6A: Multidimensional Scaling plot based on Y-STR allelic frequency data for individuals possessing haplogroup E1b1a7a-U174 derivatives.... 
Fig. 6B: Multidimensional Scaling plot based on Y-STR allelic frequency data for individuals possessing haplogroup E1b1a8-U175 derivatives. 


\section{LIST OF TABLES}

TABLE

PAGE

\section{Chapter II}

Table 1: Populations Analyzed

Table 2: Admixture analysis for New Providence and two African American groups from the US

Table 3: Admixture analysis of Latin American populations ........................................34

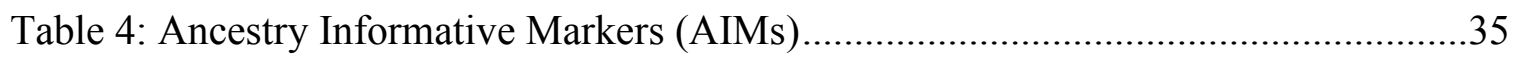

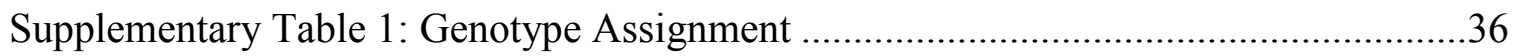

Supplementary Table 2: New Providence Allelic Frequencies $(n=221)$.......................42

Supplementary Table 3: Forensic parameters for the New Providence population

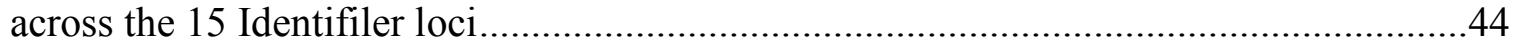

Supplementary Table 4: Total, inter- and intra-population genetic variance ..................45

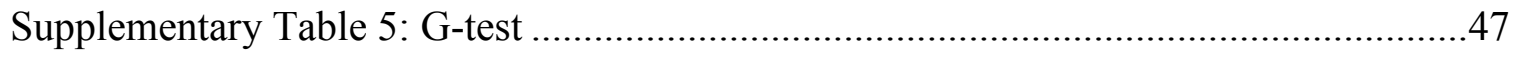

\section{Chapter III}

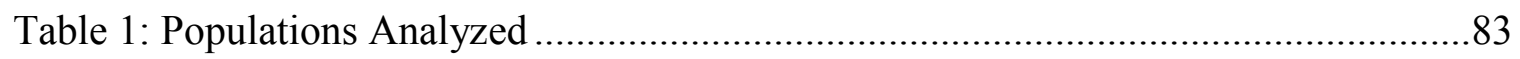

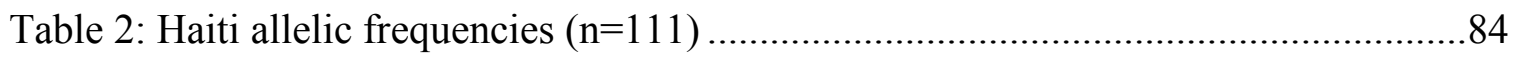

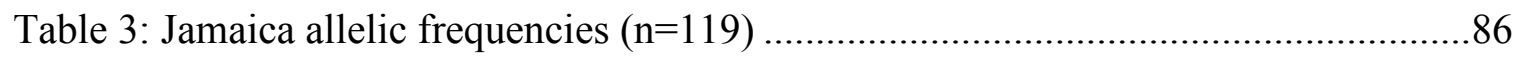

Table 4: Observed $\left(\mathrm{H}_{\mathrm{o}}\right)$ and Expected Heterozygosity $\left(\mathrm{H}_{\mathrm{e}}\right)$, Hardy-Weinberg Equilibrium (HWE) P-values and Gene Diversity indices (GDI) for the Haitian and Jamaican populations across the 15 Identifiler loci

Table 5: Inter-, intra- and total population genetic variance......................................... 88

Table 6: Heterozygote deficiencies for the Afro-Caribbean populations ........................90

Table 7: Delta $(\delta)$ values for the grouped East vs. West African populations...................91 
Table 8: G-test...

Table 9: Admixture analyses for the New World African populations .96

Supporting Information Table 1: Admixture estimates generated for slow vs. fast mutating loci

\section{Chapter IV}

Table 1: Populations analyzed .130

Table 2: Parameters of forensic and population genetics interest.

Table 3: Intra-population diversity indices for the Bahamian populations

Table 4: Inter-, intra- and total population genetic variance. .134

Table 5: Admixture proportions generated using grouped parental sources .136

Table 6: Membership proportions generated by Structure 136

Supplementary Table 1: Allelic Frequencies for Abaco 146

Supplementary Table 2: Allelic Frequencies for Eleuthera.

Supplementary Table 3: Allelic Frequencies for Exuma

Supplementary Table 4: Allelic Frequencies for Grand Bahama .150

Supplementary Table 5: Allelic Frequencies for Long Island .152

Supplementary Table 6: Forensic parameters for Abaco, Eleuthera, Exuma, Grand Bahama and Long Island across the 15 Identifiler loci.

Supplementary Table 7: G-test .156

\section{Chapter V}

Table 1: Populations utilized in Y-SNP analyses .203

Table 2: Frequencies of E1bla7 and E1bla8 sub-haplogroups in the Bahamian and continental African populations .205 
Table 3: Admixture proportions generated for the Bahamian and New World populations .206

Supplementary Table 1: Y-STR haplotypes for E1b1a7a individuals ..........................207

Supplementary Table 2: Y-STR haplotypes for E1b1a8 individuals...........................210

Supplementary Table 3: Y-STR haplotypes for R1b1b1 individuals ...........................213

Supplementary Table 4: Populations examined in E1b1a7a-U174 Y-STR based analyses

Supplementary Table 5: Populations examined in E1b1a8-U175 Y-STR based analyses

Supplementary Table 6: Populations examined in R1b1b1-M269 Y-STR based analyses

Supplementary Table 7: Haplotype Diversity indices for E1b1a7a-U174 individuals

Supplementary Table 8: Haplotype Diversity indices for E1b1a8-U175 individuals. .220

Supplementary Table 9: Haplotype Diversity indices for R1b1b1-M269 individuals. 


\section{INTRODUCTION}

The Bahamas is a low lying archipelago that extends from the southeastern tip of Florida to Cape Haitien, the northernmost part of Haiti. Geographically, the island chain is subdivided into three regions: the Northwest (NW), Central and Southeast (SE) Bahamas (Albury, 1975) (Fig. 1). While only Grand Bahama, Abaco, Bimini and the Berry Islands comprise the NW portion of the archipelago, the more populous Central Bahamas consists of (from north to south) Andros, New Providence, Eleuthera (including Harbor Island and Spanish Wells), Exuma and its cays, Cat Island, Long Island, San Salvador, Rum Cay and Ragged Island. Like the NW Bahamas, the SE Bahamas is formed by a smaller subset of islands (Crooked Island, Acklins, Mayagua and Inagua) but in contrast to the other two regions, the population size is substantially lower, totaling a mere 2,006 individuals (Department of Statistics of the Bahamas, 2000).

Historical records indicate that the indigenous people of the Bahamas, i.e., the Arawak (Lucayan) Indians (Bahamas Dept. of Archives, 1996; Johnson 1996), settled throughout the archipelago during the Ostionoid expansion (600-1100 AD). This group of Amerindians is believed to have traveled north from the Amazon River Basin into the Lesser and then Greater Antilles, arriving in the Bahamas around 800 AD (Keegan, 1992; Keegen, 2000). The Lucayans resided on the island of Guanahani (believed to be presentday San Salvador) until the invasion of the Spanish, who deported them to Hispaniola and Cuba where they were enslaved (Bahamas Dept. of Archives, 1996; Wilkie and Farnsworth, 2005). By the mid 1500s, most of the Lucayans had perished (Granberry, 1981; Johnson, 1996) and the Bahamas became virtually uninhabited (Miller, 1945; Lawlor, 1998). 
Although claimed by the British Empire in 1629 (Turner, 2003), the first permanent settlement of the islands did not occur until 1648 by the Eleutherian Adventurers, a group of Puritan pilgrims (Lawlor, 1998). The pilgrims fled England to escape religious persecution and after continued religious turmoil in Bermuda (the first place where they sook refuge), the Adventurers landed on Cigatoo (present-day Eleuthera) with the hope of establishing a truly independent colony in the Bahamas (Miller, 1945; Saunders, 1991). They were subsequently joined by other exiled Bermudian colonists that would not take oath to the new king, Charles II (Craton, 1986; Lawlor, 1998). The Bermudian period (1648-1670) in the Bahamas ended with most Puritans returning to Bermuda as conditions improved, leaving a few hundred individuals behind (Wilkie and Farnsworth, 2005).

In the following century, the Bahamas was raided over thirty times by French and Spanish pirates (Saunders, 1990), and in the course of power struggles among the European conquerors (i.e., France, Spain, Britain and the Netherlands) for domination of the West Indies, was finally recaptured by Spain in 1782 (Williams, 1990). The signing of the Treaty of Versailles in 1783, however, brought an end to the American Revolutionary War and granted Florida to Spain and the Bahamas to the British Empire (Williams, 1983). As a result, thousands of British Loyalists fled continental North America for the Bahamas between 1783 and 1785 ("the Bahamian Revolution") (Craton, 1986). The Bahamas was chosen as a suitable refuge since the islands remained under British rule and, as an additional incentive, each Loyalist was promised several acres of unoccupied land by the Crown (Saunders, 1978). 
The migration of the Loyalists into the island chain greatly impacted Bahamian society. Prior to their arrival, only New Providence, Eleuthera, Harbour Island, Exuma and Cat Island were permanently settled and individuals of African and European descent were almost equally represented in number (Saunders, 1985). Yet, by 1788 the population had doubled, with Africans outnumbering Europeans 2:1, and the number of inhabited islands had expanded to include Abaco, Andros, Crooked Island and Long Island (Sharer, 1955). The escalation in the African component resulted from the slaves of Creole descent [an individual of African ancestry that is born in the New World (Saunders, 1985)] that accompanied the Loyalists and the importation of African born individuals in the following years to meet the increased demand for plantation laborers (Albury, 1975; Johnson, 1996). Additionally, large numbers of liberated African slaves captured by the British Royal Navy were resettled in the Bahamas following the abolition of the slave trade in 1807 . Thereafter, the island chain was influenced by various ethnic groups, including Greeks, Chinese, Lebanese, Eastern European Jews (Johnson, 1991; McCartney, 2004), Jamaicans, Turks and Caicos islanders, Guyanese, Barbadians and Haitians (Tinker, 1998), who further contributed to the diverse collage-like populace of the archipelago.

On the basis of those historical accounts, it is not surprising that the deepest roots set within the Bahamas are attributed to African and European sources. Junkanoo, for example, is a festival that is believed to have originated from a West African slave leader by the name of "John Canoe." Several elements derived from his culture, including the masks, which are similar to those worn by the Egunguns (a Yoruba tribe), and goombay music, a melodic mixture of rhythmic drumming [the goat skin drum used resembles the 
West African djembe drum (Thompson, 2010)] and folk songs (Baker, 2001), are the basis of this important cultural celebration. In addition to Junkanoo, obeah (black and white magic), religion, dance (Ring play), 'bush' medicine, folklore and the 'Asue' (the communal practices of money management) are other predominantly African customs that are still widespread throughout the archipelago (Saunders, 1995). On the contrary, European traditions (e.g., Plaiting of the Maypole) are less commonly practiced and, in most instances, are amalgamated with those derived from Africa. One of the most wellknown examples is Rake ' $n$ ' Scrape music, a unique sound produced by an accordion (of European origin) in concert with a carpenter's saw and a goombay drum (of African origin) (Ingraham, 2007).

Despite the wealth in historical and anthropological data, the amount of genetic information currently available on the Bahamas is limited. In the earliest report, Halberstein and colleagues (1981) characterized abnormal hemoglobin variants (Hb S, C and F) among the people residing in Bimini, suggesting that their presence is likely attributed to African influx into the island. In another study based on the D1S80 polymorphism, the Bahamas clustered with three African American groups from the United States (US) in an unweighted pair group method with arithmetic mean (UPGMA) tree, indicating that the Bahamian population is indeed of African origin (Duncan et al., 1996). Similar findings were also reported by Herrera and collaborators (2004), however, in their study, the Bahamian populace not only clustered with the collection from Haiti but also with the those from Benin, Cameroon, Congo, Kenya, Nigeria, Rwanda and Zimbabwe in both global principal component (PC) and maximum likelihood (ML) phylogenies. In addition to the D1S80 locus, Budowle et al. (1999) reported allele 
frequencies for the 13 Combined DNA Index System (CODIS) Short Tandem Repeat (STR) loci in the general Bahamian population, amongst five other groups from the New World, in order to establish a genetic database for forensic purposes. Unfortunately, in these studies, no attempt has been made to genetically characterize any of the Bahamian islands (outside of Bimini) independently.

The work in Chapter II was undertaken to ascertain the genetic composition and phylogeny of the current population in New Providence since the majority of the Bahamian populace (69.4\%) resides on this island (Department of Statistics of the Bahamas, 2000). In this study, the 15 autosomal STR loci included in the AmpFlSTR ${ }^{\circledR}$ Identifiler kit (Applied Biosystems, Foster City, CA) were analyzed in the collection from New Providence and subsequently compared to published reference populations available from the literature. The results suggest a strong genetic affinity between the New Providence collection and two African American groups from the US, most likely attributed to the migration of the Loyalists and their slaves from continental North America. All three populations also appear to be genetically more similar to the reference West African collections (particularly Equatorial Guinea and Angola) than to the East African groups, possibly because of high degrees of gene flow from West Africa into the New World during the Transatlantic Slave Trade (Simms et al., 2008)

Given the lack of Caribbean reference populations available for phylogenetic comparisons to the Bahamian collections, autosomal STR datasets for two other AfroCaribbean populations (Haiti and Jamaica) were compiled in Chapter III. Using the allelic frequency data generated across the 15 Identifiler loci, the Haitian and Jamaican collections were subsequently compared to worldwide geographically targeted reference 
populations to survey the genetic diversity present in each island. In this study, I have found that although both Haiti and Jamaica display genetic affinities with the continental African collections, a much stronger African signal is detected in Haiti. Jamaica, on the other hand, exhibits an admixture profile similar to other New World collections of African descent analyzed in this report, with comparable levels of genetic input from European and East Asian sources. The divergent genetic signatures present in these populations allude to the different migratory events of Africans, Europeans, and East Asians into the New World (Simms et al., 2010).

Chapter IV focuses on the establishment of anthropologically well-characterized forensic databases for several of northwestern (Grand Bahama and Abaco) and central (Eleuthera, Exuma and Long Island) Bahamian islands across the 15 Identifiler loci. A second aim of the study is to ascertain the genetic distribution and phylogenetic relationships of each island population as well as their genealogies within the context of diverging migratory scenarios, settlement patterns, ethnic influences and present social structuring (Bethel, 2002), which are all postulated to have contributed to the highly diverse, collage-like populace of the island chain. Altogether, my findings suggest that Bahamians are a highly heterogeneous group, with each island sampled receiving differential contributions from African, European, East Asian and Native American sources. Even though the strongest genetic signal in all five collections emanates from continental Africa, inter-island differentiation is apparent in the MDS, Structure and admixture analyses (Simms et al., 2011). 
In Chapter V, high-resolution Y-chromosome analyses were performed to delineate the patriarchal ancestry of the six Bahamian islands sampled (i.e., Abaco, Eleuthera, Exuma, Grand Bahama, Long Island and New Providence) and their genetic relationships with previously published reference populations. The results identify genetic signals emanating exclusively from African and European sources, with haplogroups E-M96 and R-M306 accounting for greater than 85\% of all Bahamian patrilineages. The major difference among the islands lies in the opposing distribution of sub-haplogroups E1b1a-M2 and R1b1b1-M269, with the former predominating Ylineages in the collections from Abaco, Exuma, Eleuthera, Grand Bahama and New Providence while the latter is found at elevated levels in the Long Island populace. Substantial inter-island variation is also noted in the Y-STR based analyses, especially with respect to E1b1a7a-U174 and E1b1ba8-U175 sub-lineages. This Chapter is written in the format required by the American Journal of Physical Anthropology. 


\section{REFERENCES}

Albury, P. 1975. The story of the Bahamas. London: Macmillan Education Limited.

Department of Statistics of the Bahamas, The 2000 census of population and housing tables (All Bahamas). http://statistics.bahamas.gov.bs/archives.php?cat=70.

Bahamas Department of Archives. 1996. The Peoples of the Bahamas. Nassau, Bahamas: Government Printing Department.

Baker CP. 2001. Bahamas, Turks \& Caicos. Victoria, Australia: Lonely Planet Publications Pty Ltd.

Budowle B, Moretti T, Baumstark AL, Defenbaugh DA, Keys KM. 1999. Population data on the thirteen CODIS core short tandem repeat loci in African Americans, U.S. Caucasians, Hispanics, Bahamians, Jamaicans, and Trinidadians. J Forensic Sci 44:12771286.

Craton M. 1986. A history of the Bahamas. Ontario, Canada: San Salvador Press.

Dalleo PT. 1982. African-Bahamian origins. J Bahamas Hist. Soc 4:17-19.

Dalleo PT. 1984. Africans in the Caribbean: a preliminary assessment of recaptives in the Bahamas 1811-1860. J Bahamas Hist Soc 6:15-24.

Duncan G, Thomas E, Gallo JC, Baird LS, Garrison J, Herrera RJ. 1996. Human phylogenetic relationships according to the D1S80 locus. Genetica 98:277-287.

Granberry J. 1981. Spanish slave trade in the Bahamas, 1509-1530: an aspect of the Caribbean pearl industry (Last Part). J Bahamas Hist Soc 3:17-19.

Halberstein RA, Davies JE, Mack K. 1981. Hemoglobin variations on a small Bahamian island. Am J Phy Anthropol 55:217-221.

Herrera RJ, Adrien LR, Ruiz LM, Sanabria NY, Duncan G. 2004. D1S80 single-locus discrimination among African populations. Hum Biol 76:87-108.

Johnson H. 1991. The Bahamas in slavery and freedom. Kingston, Jamaica: Ian Randle Publishing.

Johnson H. 1996. The Bahamas from slavery to servitude, 1783-1933. Gainesville, Florida: University Press of Florida.

Keegan WF. 1992. The people who discovered Columbus. Gainesville, Florida: University Press of Florida. 
Keegan W. 2000. West Indian archeology. 3. Ceramic age. J Archaeol Res 8:135-167.

Ingraham V. 2007. The Bahamas. In: Kuss M, editor. Music in Latin America and the Caribbean: performing the Caribbean experience, an encyclopedic history. Austin, TX: University of Texas Press. p 359-376.

Lawlor A. 1998. The Eleutherian Adventurers. J Bahamas Hist Soc 20:4-9.

McCartney DM. 2004. Bahamian culture and factors which impact upon it. Pittsburg, PA: Dorrance Publishing Co., Inc.

Miller WH. 1945. The colonization of the Bahamas, 1647-1670. The William and Mary Quarterly $3^{\text {rd }}$ Ser 2:33-46.

Saunders, DG. 1978. The slave population of the Bahamas 1783-1834. Dissertation: University of the West Indies.

Saunders G. 1985. Slavery in the Bahamas 1648-1838. Nassau, Bahamas: The Nassau Guardian.

Saunders G. 1990. Life in New Providence in the early eighteenth century. J Bahamas Hist Soc 12:9-14.

Saunders G. 1991. The early settlers in the Bahamas. J Bahamas Hist Soc 13:13-17.

Saunders G. 1995. Aspects of traditional African-Bahamian culture in the later $19^{\text {th }}$ and early $20^{\text {th }}$ century. J Bahamas Hist Soc 17:2-10.

Simms TM, Garcia C, Mirabal S, McCartney Q, Herrera RJ. 2008. The genetic legacy of the Transatlantic Slave Trade in the island of New Providence. Forensic Sci Int: Genet 2:310-317.

Simms TM, Rodriguez CE, Rodriguez R, Herrera RJ. 2010. The genetic structure of populations from Haiti and Jamaica reflect divergent demographic histories. Am J Phys Anthropol 142:49-66.

Simms TM, Barrett DA, McCartney Q, Herrera RJ. 2011. Divergent genetic strata in five Bahamian islands. In Press Forensic Science International: Genetics.

Thompson K. 2010. Caribbean islands: the land and the people. Dar es Salaam, Tanzania: New Africa Press.

Tinker KL. 1998. Perspectives on West Indian migration to the Bahamas: pre-Columbian to Bahamian independence in 1973. Dissertation: Florida State University. 
Turner G. 2003. The Bahamas in the early 1700s: a pirate haven brought under rule. J Bahamas Hist Soc 25:4-9.

Wilkie L, Farnsworth P. 2005. Sampling many pots. Gainesville, Florida: University Press of Florida.

Williams P. 1983. The Loyalists and their settlements. J Bahamas Hist Soc 5:11-15.

Williams PM. 1990. War and its consequences: the Bahamian experience 1700-1763. J Bahamas Hist Soc 12:15-19. 


\section{Appendix I}

Fig. 1: Map of the Bahamas illustrating the islands comprising the northwest, central and southeast portions of the archipelago

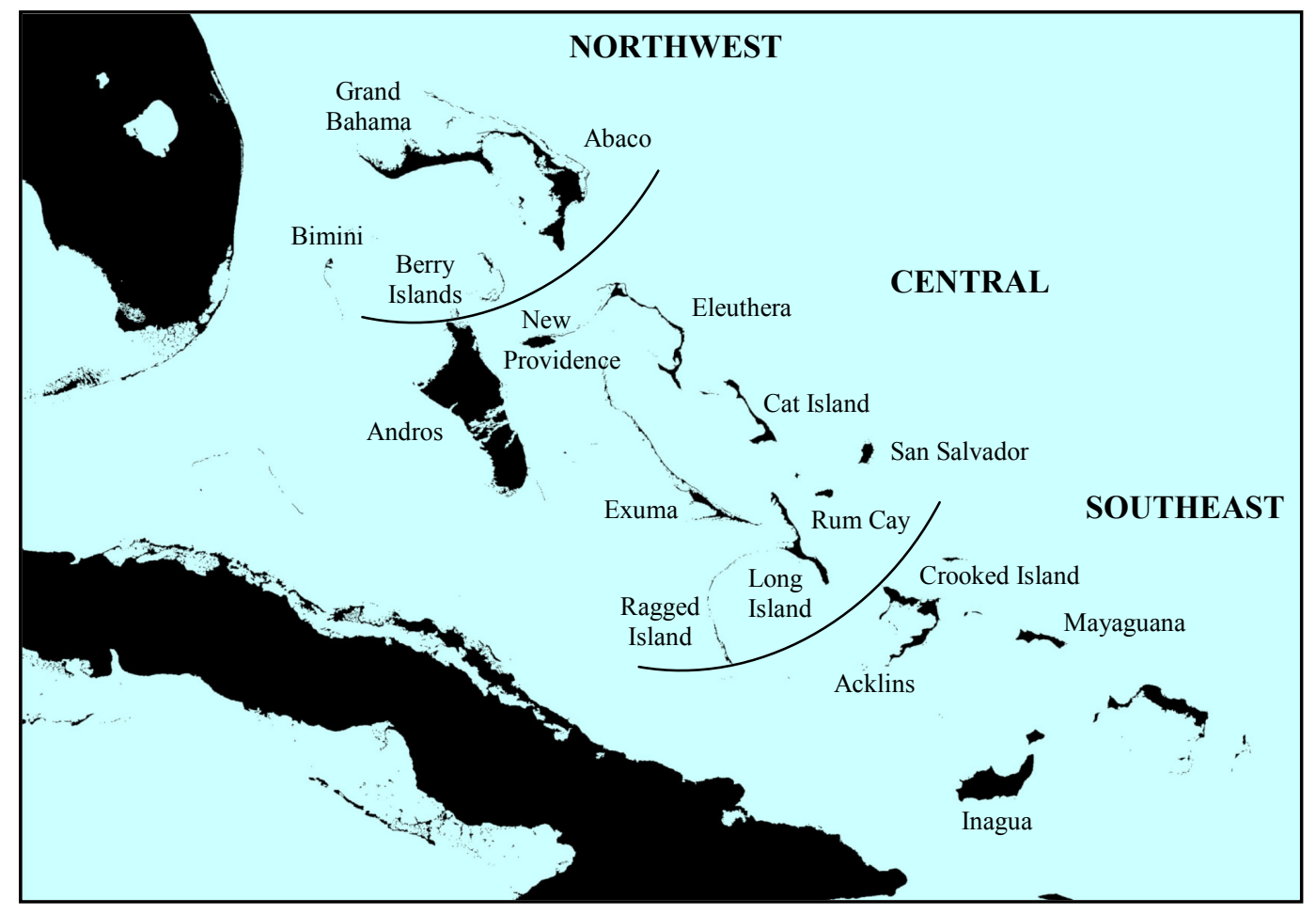




\section{THE GENETIC LEGACY OF THE TRANSATLANTIC SLAVE TRADE IN THE ISLAND OF NEW PROVIDENCE}

\section{A. Introduction}

The number of genetic studies characterizing the populations of the Bahamas is limited. For instance, Halberstein et al. [1] examined abnormal hemoglobin variants among the people residing on the island of Bimini while Duncan et al. [2] reported D1S80 polymorphisms in the general populace of the Bahamas. A more recent study by Budowle et al. [3], reported the allelic frequencies of the 13 CODIS STR loci in a collection of individuals from different Bahamian islands, in an attempt to establish a genetic database for forensic purposes. Considering the complex demographic landscape generated by unique ancestral populations that have contributed genetically to the island chain, the need for insular-specific population studies and datasets for forensic casework is paramount.

The present work was undertaken to ascertain the genetic diversity in the current population of the island of New Providence in the Bahamian archipelago and the discrimination provided by 15 autosomal STR markers routinely employed in forensic analyses. Autosomal STRs provide for the high resolution required for assessing individual identity in forensic cases for inclusion and exclusion determination [4]. The increased number of loci analyzed and larger number of anthropologically wellcharacterized individuals examined in this study, allow for robust probability determinations and greater scientific certainty in forensic testing than previously achieved. 


\section{B. Materials and Methods}

\section{Sample Collection and DNA Extraction}

Buccal swabs were collected with informed consent from 221 individuals residing in the island of New Providence, Bahamas (Fig. 1A). All ethical guidelines were followed as set by the Florida International University Institutional Review Board (IRB). DNA was extracted according to the manufacturers' instructions (Qiagen Inc., Valencia, CA; Puregene, Gentra Systems, Minneapolis, MN) and stored at $-80^{\circ} \mathrm{C}$.

\section{Published Data}

A total of 29 data sets from published geographically targeted collections [5-30] (Table 1) were included as reference populations for comparisons to the New Providence group. The geographical origins of the reference African populations as well as known ports of embarkation are provided in Fig. 1B.

\section{DNA Amplification and STR Genotyping}

PCR amplification of the 15 autosomal STRs (D8S1179, D21S11, D7S820, CSF1PO, D3S1358, TH01, D13S317, D16S539, D2S1338, D19S433, vWA, TPOX, D18S51, D5S818 and FGA) was performed utilizing the AmpFlSTR ${ }^{\circledR}$ Identifiler kit (Applied Biosystems, Foster City, CA) according to the manufacturer's specifications. Amplified products were separated and scored as previously published [6,31]. 


\section{Statistical and Phylogenetic Analyses}

Allelic frequencies, several parameters of forensic and population genetics interest [Matching Probability (MP), Power of Discrimination (PD), Polymorphic Information Content (PIC), Power of Exclusion (PE), Gene Diversity Index (GDI) and Typical Paternity Index (TPI)], expected and observed heterozygosities $\left(\mathrm{H}_{\mathrm{e}}\right.$ and $\mathrm{H}_{\mathrm{o}}$, respectively), and Hardy-Weinberg equilibrium (HWE) $P$-values were assessed as previously reported [6,31]. Inter-, intra- and total population variance $\left(\mathrm{G}_{\mathrm{st}}, \mathrm{H}_{\mathrm{s}}\right.$ and $\mathrm{H}_{\mathrm{t}}$, respectively) were estimated with the DISPAN program [6,31]. The collections examined in this analysis were assembled according to bio-geographical origin as shown in Table 1.

The Carmody's G-test program and Bonferroni adjustment $(\alpha=0.05 / 378=$ 0.0001323 ) were utilized to ascertain whether pair-wise population comparisons yield statistically significant differences through 100,000 simulations [6,31]. In addition, correspondence analysis (CA) $[6,31]$ and neighbor-joining (NJ) [31] dendrograms were generated as previously indicated. Ancestry informative markers (AIMs) were also assessed as described in earlier studies [6,31].

Admixture analyses were performed with the Statistical Package for the Social Sciences (SPSS) 14.0 software [6,31]. Parental groups were constructed according to biogeographic origin (Table 1) and for the Afro-American populations, the parental African

reference collections were partitioned into West (Angola and Equatorial Guinea) and East (Kenya, Madagascar, Mozambique, Rwanda Hutu and Rwanda Tutsi) African groups. 


\section{Results}

Intra-Population Diversity

Genotype assignment and allelic frequencies for the 15 autosomal STR loci typed in the New Providence population are listed in Supplementary Tables 1 and 2, respectively, while $\mathrm{H}_{\mathrm{o}}, \mathrm{H}_{\mathrm{e}}$ and HWE $P$-values are provided in Supplementary Table 3 along with the parameters of population genetics and forensic interest. Four loci, CSF1PO, D2S1338, D19S433 and TPOX, were found to deviate from HWE expectations, however, these departures became statistically insignificant after applying the Bonferroni correction $(\alpha=0.05 / 15=0.0033)$. GDI values illustrate that the D13S317 locus (0.7251) exhibits the lowest variability out of the 15 loci tested while D2S1338 $(0.8885)$ possesses the highest within the population. Furthermore, the locus with the highest PD was D2S1338 (0.9723) whereas the FGA locus was found to be the most informative for PE (0.8242) analyses. Overall, the combined matching probability is 1 in $6.99 \times 10^{17}$ and the Combined PE is 0.999998696.

Although present at low frequencies in the New Providence collection, alleles D16S539-6 and FGA-44.2 are shared exclusively with the West African population of Angola as well as allele FGA-16.1 with the Madagascar populace. New Providence and the general US African American collection (US2) share allele 30.2 at the FGA locus with Angola, Equatorial Guinea, Mozambique and the Rwanda Hutus. The same US African American group was also found to exhibit alleles D21S11-38 and D18S51-16.2 characteristic of the East African groups, i.e., Mozambique and the Rwanda Hutus, 
respectively. All three Afro-American populations also carry allele 19.2 at the FGA locus, present in Kenya, Mozambique and the Rwanda Hutus.

Intra-population variance $\left(\mathrm{H}_{\mathrm{s}}\right)$ values are provided in Supplementary Table 4. The Afro-American assemblage (0.80205) exhibits the highest intra population variance while the lowest $\mathrm{H}_{\mathrm{s}}$ levels are observed in the Asian collections (0.78103).

\section{Inter-Population Diversity}

Assessment of the phylogenetic relationships among populations was accomplished by performing a G-test, CA, NJ and admixture analyses. Three welldelineated groupings are evident in the CA plot (Fig. 2): an African group in the lower right quadrant, a European/Latin American partition in the upper left section of the graph and an Asian cluster in the lower left portion. The New Providence and US African American populations fall within the African cluster and map closer to each other than to the rest of the African collections (Fig. 2).

The topology of the NJ phylogram (Fig. 3) reveals a tight clustering of populations according to bio-geographic origin. An African branch is situated at the upper portion of the dendrogram while the other three clades (Asian, Latin American and European) are located toward the bottom of the projection. Also evident from the tree is the intermediate partitioning of the New Providence, US African American 1 and 2, Madagascar, Brazil and Puerto Rican American populations between the African groups and the other three clusters. 
The highest inter-population variance $\left(\mathrm{G}_{\mathrm{st}}\right)$ is observed in the All populations (0.02403) group and lowest in the Afro-American collections (0.00208) while total variance $\left(\mathrm{H}_{\mathrm{t}}\right)$ is highest within the Afro-American group (0.80372), with the exception of the All populations group (0.80987), and lowest in the European populations (0.78737). Statistically insignificant genetic differences in the pair-wise analyses (G-test, See Supplementary Table 5) are shown in italics before and in bold after applying the Bonferonni correction.

Admixture proportions (Table 2) for the New Providence population, when analyzed against grouped parentals, reveal that $83.6 \%$ of its gene pool is contributed by the African groups while the remaining genetic influences are from the Europeans (16.3\%). Similar results were also obtained for both the US African American 1 (Minnesota) and US African American 2 (general) populations (Table 2): African $(84.1 \%, 83.8 \%)$ and European $(15.9 \%, 14.6 \%)$, respectively. With regard to the African reference populations, the admixture proportions (Table 2) demonstrate that the West African collections (Angola and Equatorial Guinea) make a greater contribution (46.8\%, $56.1 \%$ and $48.5 \%$, respectively) to all three Afro-American populations than do the East African groups (Kenya, Madagascar, Mozambique, Rwanda Hutu and Tutsi) (36.8\%, $28.0 \%$ and $35.3 \%$, respectively). Furthermore, admixture estimations performed for each of the individual Latin American collections (Table 3) reveal that European contributions to each group's gene pool reach levels greater than $48 \%$ while the remaining influences are of African and to a lesser extent Asian descent. 
Assessment of AIMs delineates TH01, TPOX and D18S51 as particularly informative loci for African ancestry while D8S1179, D2S1338 and D19S433 are elucidative for both African and European descent (Table 4).

\section{Discussion}

In the present study, 15 autosomal STR loci, routinely employed in forensic analyses, were typed in order to examine the genetic diversity and assess the genetic ancestry of the New Providence population. The increased number of hypervariable markers analyzed along with a larger number of individuals typed improves upon earlier studies by providing a higher resolution for defining phylogenetic relationships and an anthropologically well-characterized database for forensic purposes including exclusion and inclusion determinations.

The New Providence and US African American 1 and 2 collections exhibit 173, 162 and 168 alleles, respectively, while on average, the mainland sub-Saharan African populations included in this study possess 147 alleles. This high diversity level is likely the result of the combined contributions of European and African DNA known to have occurred in these populations $[32,33]$. These results are corroborated by the average heterozygosity values of the three Afro-American collections (Table 1) which are comparable to the collective average heterozygosity of the continental African groups (0.7974) (excluding South Africa since $\mathrm{H}_{\mathrm{o}}$ values were not provided in the original report) examined in this study (Table 1). Of the three Afro-American populations, the Minnesota US African American collection exhibits the highest average heterozygosity value (0.80380) while New Providence possesses the lowest (0.78311). The relatively 
lower average heterozygosity seen in the New Providence collection, in combination with the $3.87 \%$ (Average) heterozygote deficiency based on 12 loci (Supplementary Table 3) may reflect inbreeding given its insular geographical isolation.

The comparability in the level of genetic diversity between the Afro-American populations and the mainland African collections is also apparent upon examination of the intrapopulation diversity values $\left(\mathrm{H}_{\mathrm{s}}\right)$. At the intra-population level, the AfroAmerican assemblage harbors the highest $\mathrm{H}_{\mathrm{s}}$ value (0.80205) of all groups examined, even higher than the African populations, including (0.79543) and excluding (0.79474) Madagascar (Madagascar was excluded in one of the African sets to ascertain the effect, if any, of Austronesian gene flow [6]), possibly the result of admixture of several founder populations, for example the Loyalists and Africans [32,34]. Moreover, comparison of the Afro-American assemblage with the reference collections in this study reveals that these groups possess certain specific signature alleles at the D16S539 (allele 6), D18S51 (allele 16.2), D21S11 (allele 38) and the FGA (alleles 16.1, 19.2, 30.2 and 44.2) loci characteristic of sub-Saharan African groups, suggesting a connection between the two.

Additionally, AIM analysis reveals diagnostic loci (TPOX, D18S51, D8S1179, D2S1338, D19S433 and TH01) for all populations of African descent, including the three Afro-American populations. These loci set the African collections apart from other worldwide bio-geographic groups and therefore represent pharos of gene flow from Africa into the New World. 
The G-test results also indicate genetic similarities between New Providence and both US African American populations consistent with an earlier study based on the D1S80 locus in which a general collection from the Bahamas was found to cluster in a UPGMA tree with four African American populations [2]. The New Providence and US African American collections also display insignificant genetic differences with subSaharan African groups (Supplementary Table 5). The high degree of homogeneity among the Afro-American populations is also reflected at the inter-population variance level, with the assemblage exhibiting the lowest $\mathrm{G}_{\mathrm{st}}$ value of all groups examined (Supplementary Table 4).

The partitioning of the two US African American and New Providence collections together at the periphery of the African cluster and in the direction of the European/Latin American assemblage in the CA graph suggests European genetic contributions to these New World populations while still advocating close genetic ties to mainland African groups. The intermediate positions of New Providence and US African American 1 and 2 close to the sub-Saharan populations as well as the segregation of Brazil and Puerto Rican Americans further away from the African populations in the NJ tree argues for various degrees of African genetic influence to these ethnic groups. In the present study, utilizing bi-parental hypervariable markers, the African mainland was found to contribute $41.0 \%$ and $34.4 \%$ to the Brazil and Puerto Rican American collections, respectively, while contributing on average only $18.5 \%$ (ranging from $10.3 \%$ to $27.3 \%$ ) to the other Latin American groups (Table 3). 
The admixture proportions (Table 2) generated when West and East African groups were used separately as parents for the New Providence population indicate that the majority of the influence to the collection's gene pool is from West Africa (46.8\%) followed by East Africa (36.8\%) and Europe (16.3\%). The greater impact made by the West African populations most likely is indicative of the proportion of slaves captured from the various West African regions and shipped to the Bahamas, as reported in Pepin [35]. Most of the slaves that entered the Bahamas are believed to have been transported from West African ports (Fig. 1B). Duncan et al. [2] report that the majority of slaves shipped to the Bahamas were specifically from Nigeria, Ghana and Congo. Other sources indicate that the Africans settling in the New World were from Angola, the Bight of Biafra [33] and the East Coast of Africa [36]. The West African influences in the island chain are supported by a D1S80 study in which the Bahamas was found to cluster with West African groups but further away from the East African collections in a principal component (PC) plot of 33 worldwide populations [37].

Also noteworthy are the contributions detected from East Africa (36.8\%) to the New Providence populace in the admixture studies (Table 2). It is likely that the influences from this region are the result of the genetic imprints left by the Bantu diaspora (from West Africa) throughout sub-Saharan Africa [38,39]. In addition, it is possible that a portion of the contribution from South East Africans stems from the transportation of approximately $7.69 \%$ of the total African captives from the Mozambique/Madagascar region to the West African ports and from there to the New World [40,41]. 
West Africa also provides a sizeable contribution to the gene pools of US African American collections 1 and 2, 56.1\% and 48.5\%, respectively (Table 2). Hammer et al. [42] demonstrated the presence of African derived Y-chromosomes (haplogroups A, B and E) within a general US African American population, with haplogroup E (subhaplogroup E3a being a Bantu marker) exhibiting the highest frequency (62\%). A more recent study by Lind et al. [43] examining mtDNA markers corroborates the $\mathrm{Y}$ chromosome data indicating the presence of sub-Saharan African mtDNA haplogroup L (sub-haplogroups L0, L1, L2 and L3) at frequencies as high as $86 \%$ in US African Americans. The L1b sub-haplogroup (abundant throughout West Africa), for instance, is particularly prominent in US African Americans, thus supporting West African ancestry [44-46].

The New Providence and both US African American populations share similar admixture proportions $(16.3 \%, 15.9 \%$ and $14.6 \%$, respectively) from Europeans (Table 2). European influences on New Providence most likely derive, for the most part, from the influx of British Loyalists to the Bahamas during the American Revolutionary War [47]. European $\mathrm{Y}$ haplogroups I and R [42,43] and mtDNA haplogroups $\mathrm{M}$ and $\mathrm{N}[43]$ have been detected in US African American populations. In addition, only the General US African American (US2) collection exhibits contributions from the Asian groups (Table 2). Asian-specific mtDNA haplogroups A, B, C and D have been observed at low frequencies in US African American collections [33,48]. It is likely that this is partly due to admixture between US African American groups and Native Americans [33]. Also, these signals of Asian gene flow may stem from, to some extent, mainland Asian migrants ubiquitous to many New World locations. 
Although the analyses point to a strong African influence on the three AfroAmerican populations examined, differences are observed, most likely due to admixture involving different sub-Saharan African source populations as well as with non-African groups [49]. African Americans from the United States are reported as having genetic contributions from African and European ancestral populations [33], like Bahamians, but may have greater contributions from Native American populations due to the prominence of Amerindians in continental America and their early demise in the Caribbean. The Native American impact on the General US African American population (US2) may be reflected in the $1.7 \%$ contribution by Asians in the admixture analyses performed in this study. These differences are also observed when comparing each of the Afro-American populations, which reveals alleles unique to each, namely D21S11-27.1, TH01-6.3, D19S433-14.1, FGA-34.2 and FGA-46.2 present in the New Providence populace, FGA24.3 in the US Minnesota population, and D21S11-39, FGA-16.2 and FGA-22.3 in the US general African American collection. These allelic incongruencies between the New Providence and the US African American collections as well as between the two US African American populations themselves could impact on the probability of inclusion estimations when utilizing the different datasets. In turn, these allelic differences may incriminate further defendants whose STR profiles possess variants absent in nonauthentic databases from geographical regions outside the source of the crime [50]. Therefore, the presence of these rare alleles in combination with the varied ethnic distributions throughout the Bahamian islands may justify the creation of island-specific forensic datasets for these 15 autosomal STR loci. 


\section{REFERENCES}

[1] R.A. Halberstein, J.E. Davies, K. Mack, Hemoglobin variations on a small Bahamian island, Am. J. Phys. Anthropol. 55 (1981) 217-221.

[2] G. Duncan, E. Thomas, J.C. Gallo, L.S. Baird, J. Garrison, R.J. Herrera, Human phylogenetic relationships according to the D1S80 locus, Genetica 98 (1996) 277-287.

[3] B. Budowle, T. Moretti, A.L. Baumstark, D.A. Defenbaugh, K.M. Keys, Population data on the thirteen CODIS core short tandem repeat loci in African Americans, U.S. Caucasians, Hispanics, Bahamians, Jamaicans, and Trinidadians, J. For. Sci. 44 (6) (1999) 1277-1286.

[4] D.J. Rowold, R.J. Herrera, Inferring recent human phylogenies using forensic STR technology, Forensic Sci. Int. 133 (3) (2003) 260-265.

[5] E.M. Shepard, R.J. Herrera, Genetic encapsulation among Near Eastern populations, J. Hum. Genet. 51 (2006) 467-476.

[6] M. Regueiro, S. Mirabal, H. Lacau, J.L. Caeiro, R.L. Garcia-Bertrand, R.J. Herrera, Austronesian genetic signature in East African Madagascar and Polynesia, J. Hum. Genet. 53 (2007) 106-120.

[7] A.M. Gross, B. Budowle, Minnesota population data on 15 STR loci using the Identifiler ${ }^{\circledR}$ kit, J. For. Sci. 51 (6) (2006) 1410-1413.

[8] J.M. Butler, R. Schoske, P.M. Vallone, J.W. Redman, M.C. Kline, Allele frequencies for 15 autosomal STR loci on U.S. Caucasian, African American, and Hispanic populations, J. For. Sci. 48 (4) (2003) 908-911.

[9] S. Beleza, C. Alves, F. Reis, A. Amorim, A. Carracedo, L. Gusmão, 17 STR data (AmpFISTR Identifiler and Powerplex 16 System) from Cabinda (Angola), Forensic Sci. Int. 141 (2004) 193-196.

[10] A. Kido, Y. Dobashi, N. Fujitani, M. Hara, R. Susukida, H. Kimura, M. Oya, Population data on the AmpFISTR Identifilier loci in Africans and Europeans from South Africa, Forensic Sci. Int. 168 (2-3) (2007) 232-235.

[11] C. Alves, L. Gusmão, A.M. López-Parra, M.S. Mesa, A. Amorim, E. Arroyo-Pardo, STR allelic frequencies for an African population sample (Equatorial Guinea) using AmpFISTR Identifiler and Powerplex 16 kits, Forensic Sci. Int. 148 (2005) 239-242.

[12] C. Alves, L. Gusmão, A. Damasceno, B. Soares, A. Amorim, Contribution for an African autosomic STR database (AmpFISTR Identifiler and Powerplex 16 System) and a report on genotypic variations, Forensic Sci. Int. 139 (2004) 201-205. 
[13] M. Regueiro, J.C. Carril, M.L. Pontes, M.F. Pinheiro, J.R. Luis, B. Caeiro, Allele distribution of 15 PCR-based loci in the Rwanda Tutsi population by multiplex amplification and capillary electrophoresis, Forensic Sci. Int. 143 (2004) 61-63.

[14] S.P. Hu, X.J. Yu, J.W. Liu, K.L. Cai, Analysis of STR polymorphisms in the Choa Shan population in South China, Forensic Sci. Int. 147 (1) (2005) 93-95.

[15] M. Hashiyada, Y. Itakura, T. Nagashima, M. Nata, M. Funayama, Polymorphism of 17 STRs by multiplex analysis in Japanese population, Forensic Sci. Int. 133 (3) (2003) 250-253.

[16] Y. Kim, J.Y. Hwang, Y.J. Kim, S. Lee, N.E. Chung, H.E. Goh, C.C. Kim, D.W. Kim, Allele frequencies of 15 STR loci using AmpFISTR Identifiler kit in a Korean population, Forensic Sci. Int. 136 (1-3) (2003) 92-95.

[17] L.H. Seah, N.H. Jeevan, M.I. Othman, P. Jaya, Y.S. Ooi, P.C. Wong, S.S. Kee, STR data for the AmpFISTR Identifiler loci in three ethnic groups (Malay, Chinese, Indian) of the Malaysian population, Forensic Sci. Int. 138 (2003) 134-137.

[18] M.C. De Ungria, R.K. Roby, K.A. Tabbada, S. Rao-Coticone, M.M. Tan, K.N. Hernandez, Allele frequencies of 19 STR loci in a Philippine population generated using AmpFISTR multiplex and ALF singleplex systems, Forensic Sci. Int. 152 (2005) 281284.

[19] Z.Y. Wang, R.J. Yu, F. Wang, X.S. Li, T.B. Jin, Genetic polymorphisms of 15 STR loci in Han population from Shaanxi (NW China), Forensic Sci. Int. 147 (1) (2005) 8991.

[20] M.A. Chiurillo, A. Morales, A.M. Mendes, N. Lander, F. Tovar, A. Fuentes, J.L. Ramírez, Genetic profiling of a central Venezuelan population using 15 STR markers that may be of forensic importance, Forensic Sci. Int. 136 (2003) 99-101.

[21] S. Hernández-Gutiérrez, P. Hernández-Franco, S. Martínez-Tripp, M. Ramos-Kuri, $\mathrm{H}$. Rangel-Villalobos, STR data for 15 loci in a population sample from the central region of Mexico, Forensic Sci. Int. 151 (2005) 97-100.

[22] A. Rodríguez, G. Arrieta, I. Sanóu, M.C. Vargas, O. García, I. Yurrebaso, J.A. Pérez, M. Villalta, M. Sepinoza, Population genetic data for 18 STR loci in Costa Rica, Forensic Sci. Int. 168 (1) (2007) 85-88.

[23] L.P. Bernal, L. Borjas, W. Zabala, M.G. Portillo, E. Fernández, W. Delgado, F. Tovar, N. Lander, M.A. Chiurillo, J.L. Ramírez, O. García, Genetic variation of 15 STR autosomal loci in the Maracaibo population from Venezuela, Forensic Sci. Int. 161 (2006) 60-63. 
[24] A. Gorostiza, A. González-Martín, C.L. Ramírez, C. Sánchez, C. Barrot, M. Ortega, E. Huguet, J. Corbella, M. Gené, Allele frequencies of the 15 AmpFISTR Identifiler loci in the population of Metztitlán (Estado de Hidalgo), Mexico, Forensic Sci. Int. 166 (2007) 230-232.

[25] J. Zúñiga, M. Ilzarbe, V. Acunha-Alonzo, F. Rosetti, Z. Herbet, V. Romero, I. Almeciga, O. Clavijo, J.N.H. Stern, J. Granados, M. Fridkis-Hareli, P. Morrison, J. Azocar, E.J. Yunis, Allele frequencies for 15 autosomal STR loci and admixture estimates in Puerto Rican Americans, Forensic Sci. Int. 164 (2006) 266-270.

[26] A.C. de Souza Góes, D.A. da Silva, E.H. Gil, M.T. da Silva, R.W. Pereira, E.F. de Carvalho, Allele frequencies and statistic parameters for 16 STR loci - D19S433, D2S1338, CSF1PO, D16S539, D7S820, D21S11, D18S51, D13S317, D5S818, FGA, Penta E, TH01, vWA, D8S1179, TPOX, D3S1358 - in the Rio de Janeiro population, Brazil, Forensic Sci. Int. 140 (2004) 131-132.

[27] C. Coudray, R. Calderon, E. Guitard, B. Ambrosio, A. González-Martín, J.M. Dugoujon, Allele frequencies of 15 tetrameric short tandem repeats (STRs) in Andalusians from Huelva (Spain), Forensic Sci. Int. 168 (2007) e21-e24.

[28] K. Rebala, J. Wysocka, E. Kapińska, L. Cybulska, A.I. Mikulich, I.S. Tsybovsky, Z. Szczerkowska, Belarusian population genetic database for 15 autosomal STR loci, Forensic Sci. Int. 173 (2-3) (2007) 235-237.

[29] G. Mertens, N. Mommers, E. Cardoen, I. De Bruyn, E. Jehaes, S. Rand, K. Van Brussel, W. Jacobs, Flemish population genetic analysis using 15 STRs of the Identifiler ${ }^{\circledR}$ kit, Int. Congr. Ser. 1288 (2006) 328-330.

[30] J. Czarny, T. Grzybowski, M.V. Derenko, B.A. Malyarchuk, D.M. Śliwka, Genetic variation of 15 STR loci (D3S1358, vWA, FGA, TH01, D2S1338, D8S1179, D21S11, D18S51, and D19S433) in populations of north and central Poland, Forensic Sci. Int. 147 (2005) 97-100.

[31] L. Ibarrara-Rivera, S. Mirabal, M. Reguerio, R. Herrera, Delineating genetic relationships among the Maya, Am. J. Phys. Anthropol. 135 (2007) 329-347.

[32] G. Saunders, Cultural Perspectives Bahamas Department of Archives Bahamas Heritage Festival, Government Printing Department, Nassau, Bahamas, 2003, pp. 1-13.

[33] E.J. Parra, A. Marcini, J. Akey, J. Martinson, M.A. Batzer, R. Cooper, T. Forrester, D.B. Allison, R. Deka, R.E. Ferrell, M.D. Shriver, Estimating African American admixture proportions by use of population-specific alleles, Am. J. Hum. Genet. 63 (1998) 1839-1851. 
[34] H. Johnson, The Bahamas from Slavery to Servitude, 1783-1933, University Press of Florida, Gainesville, Florida, 1996.

[35] J. Pepin, From the Old World to the New World: an ecologic study of population susceptibility to HIV infection, Trop. Med. Int. Health 10 (7) (2005) 627-639.

[36] M. Childs, The Yoruba Diaspora in the Atlantic World, Indiana University Press, Bloomington, IN, 2004.

[37] R.J. Herrera, L.R. Adrien, L.M. Ruiz, N.Y. Sanabria, G. Duncan, D1S80 singlelocus discrimination among African populations, Hum. Biol. 76 (1) (2004) 87-108.

[38] S. Plaza, A. Salas, F. Calafell, F. Corte-Real, J. Bertranpetit, Á. Carracedo, D. Comas, Insights into the western Bantu dispersal: mtDNA lineage analysis in Angola, Hum. Genet. 115 (2004) 439-447.

[39] S. Beleza, L. Gusmão, A. Amorim, A. Carracedo, A. Salas, The genetic legacy of western Bantu migrations, Hum. Genet. 117 (2005) 366-375.

[40] H. Thomas, The Slave Trade - the History of the Atlantic Slave Trade: 1440-1870, Macmillan, London, 1998.

[41] L. Pereira, V. Macaulay, A. Torroni, R. Scozzari, M.J. Prata, A. Amorim, Prehistoric and historic traces in the mtDNA of Mozambique: insights into the Bantu expansions and the slave trade, Am. J. Hum. Genet. 65 (2001) 439-458.

[42] M.F. Hammer, V.F. Chamberlain, V.F. Kearney, D. Stover, G. Zhang, T. Karafet, B. Walsh, A.J. Redd, Population structure of Y chromosome SNP haplogroups in the United States and forensic implications for constructing Y chromosome STR databases, Forensic Sci. Int. 164 (1) (2006) 45-55.

[43] J.M. Lind, H.B. Hutcheson-Dilks, S.M. Williams, J.H. Moore, M. Essex, E. RuizPesini, D.C. Wallace, S.A. Tishkoff, S.J. O'Brien, M.W. Smith, Elevated male European and female African contributions to the genomes of African American individuals, Hum. Genet. 120 (2007) 713-722.

[44] A. Salas, M. Richards, T. De la Fe, M. Lareu, B. Sobrino, P. Sánchez-Diz, V. Macaulay, Á. Carracedo, The making of the African mtDNA landscape, Am. J. Hum. Genet. 71 (2002) 1082-1111.

[45] A. Salas, M. Richards, M. Lareu, R. Scozzari, A. Coppa, A. Torroni, V. Macaulay, Á. Carracedo, The African diaspora: mitochondrial DNA and the Atlantic Slave Trade, Am. J. Hum. Genet. 74 (2004) 454-465. 
[46] A. Salas, Á. Carracedo, M. Richards, V. Macaulay, Charting the ancestry of African Americans, Am. J. Hum. Genet. 77 (2005) 676-680.

[47] M. Craton, A History of the Bahamas, San Salvador Press, Ontario, Canada, 1986.

[48] E.J. Parra, R.A. Kittles, G. Argyropoulos, C.L. Pfaff, K. Hiester, C. Bonilla, N. Sylvester, D. Parrish-Gause, W.T. Garvey, L. Jin, P.M. McKeigue, M.I. Kamboh, R.E. Ferrell, W.S. Pollitzer, M.D. Shriver, Ancestral proportions and admixture dynamics in geographically defined African Americans living in South Carolina, Am. J. Phys. Anthropol. 114 (2001) 18-29.

[49] H.E. Collins-Schramm, B. Chima, T. Morii, K. Wah, Y. Figueroa, L.A. Criswell, R.L. Hanson, W.C. Knowler, G. Silva, J.W. Belmont, M.F. Seldin, Mexican American ancestry-informative markers: examination of population structure and marker characteristics in European Americans, Mexican Americans, Amerindians and Asians, Hum. Genet. 114 (2004) 263-271.

[50] D.J. Rowold, R.J. Herrera, On human STR sub-population structure, Forensic Sci. Int. 151 (1) (2005) 59-69. 


\section{Appendix II}

Fig. 1A: Map of the Bahamian archipelago indicating the island of New Providence

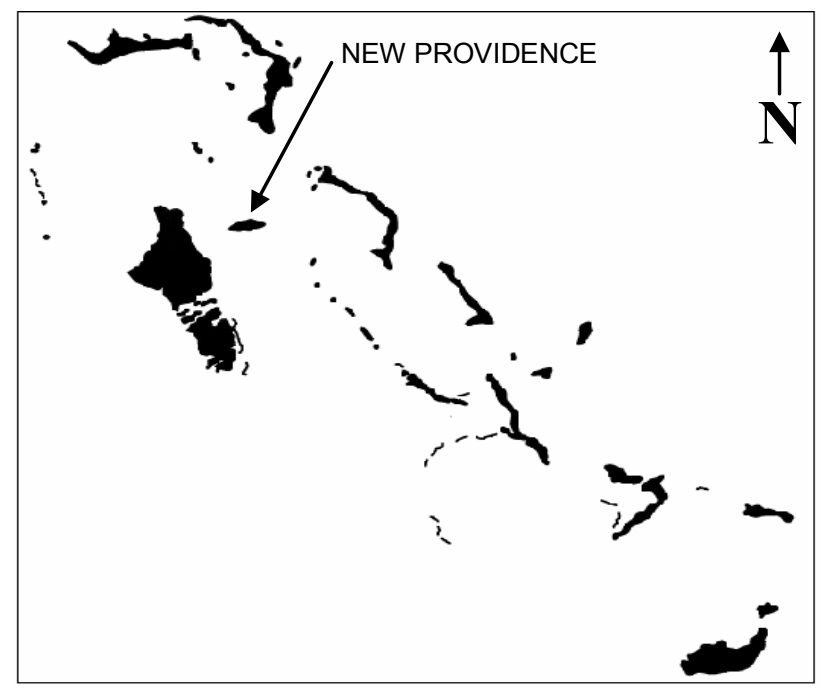


Fig. 1B: Map of Africa illustrating ports of departure (arrows) of African slaves during the Transatlantic Slave Trade and also the location of reference African collections used in the analyses

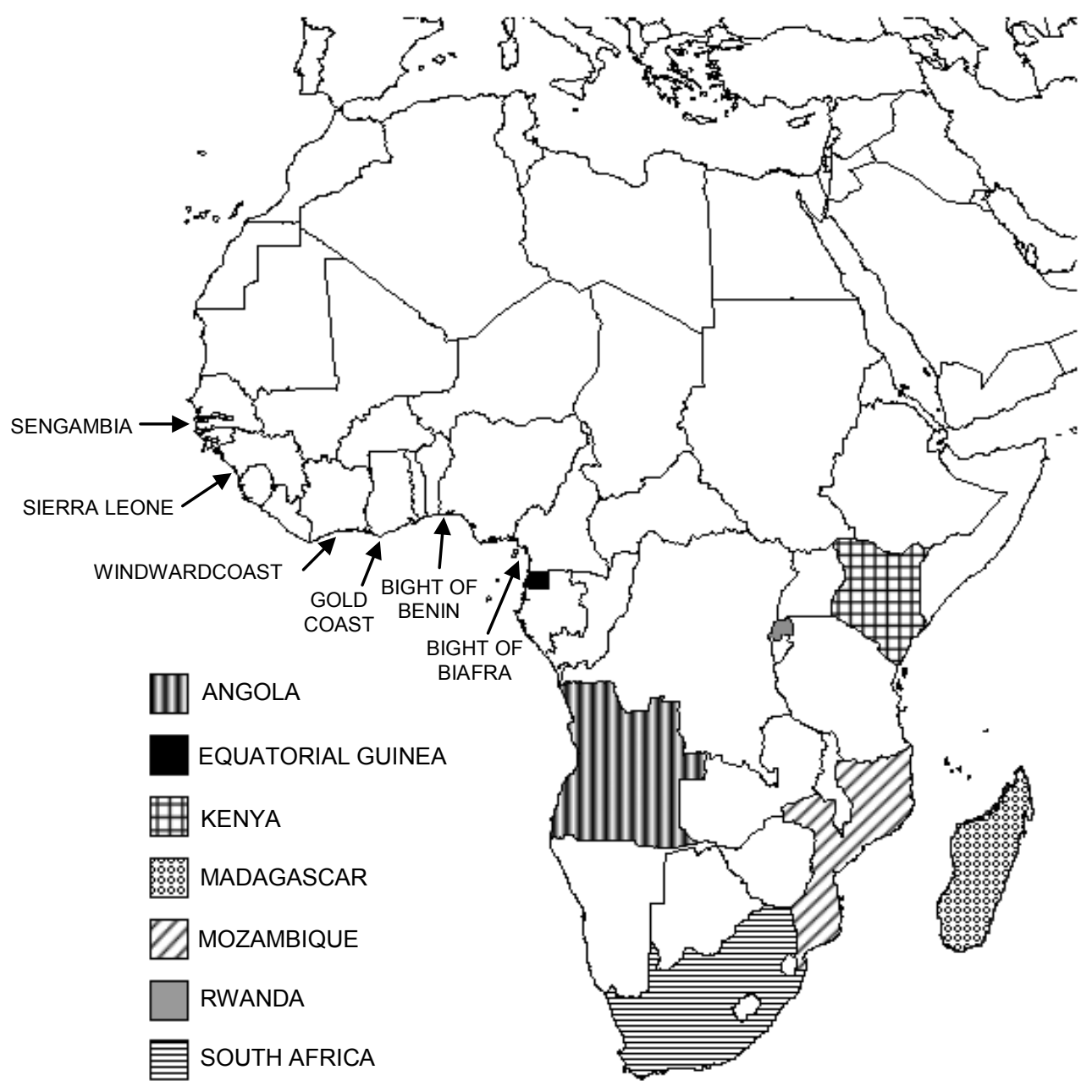


Fig. 2: Correspondence Analysis

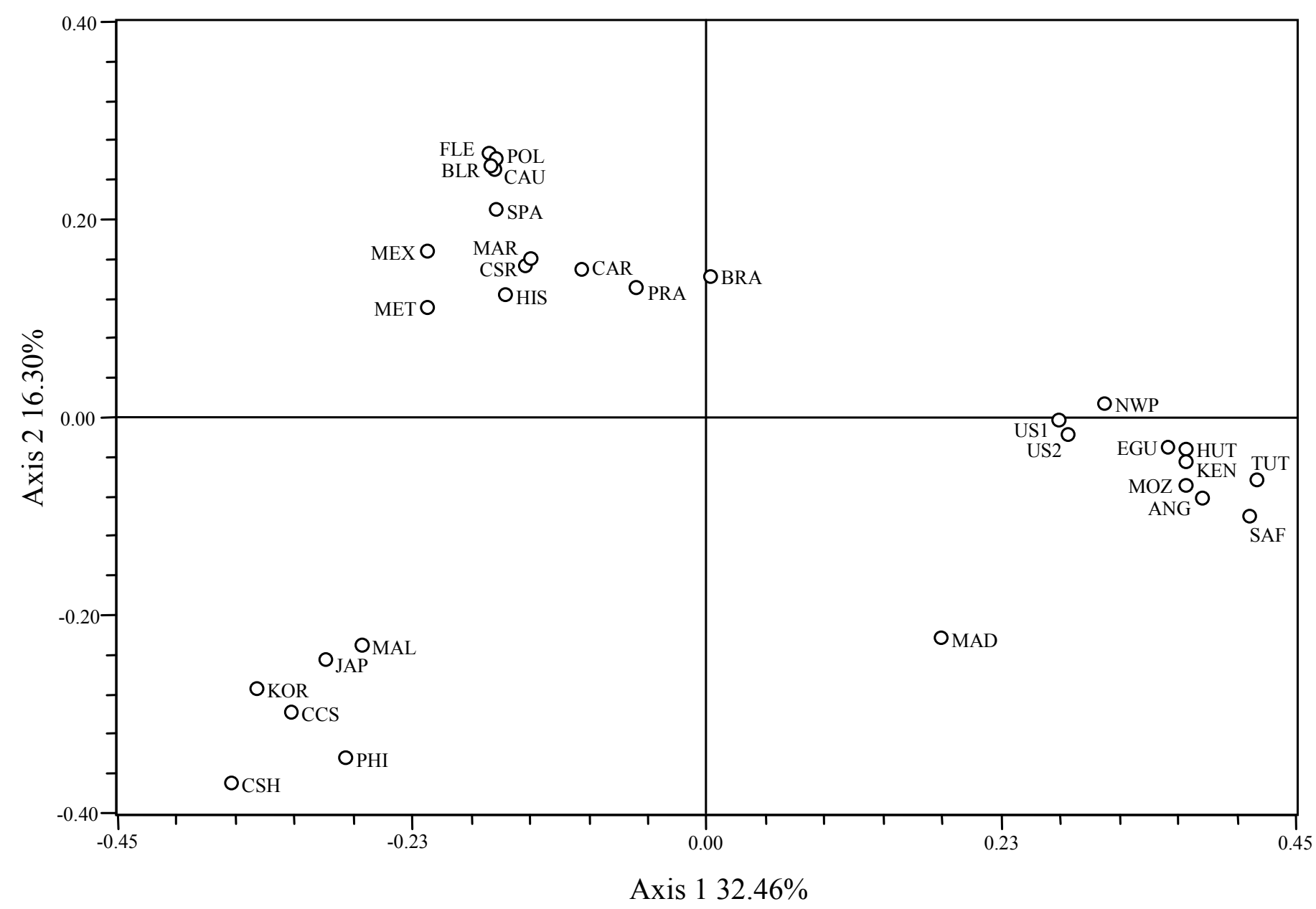


Fig. 3: Neighbor-Joining Dendrogram

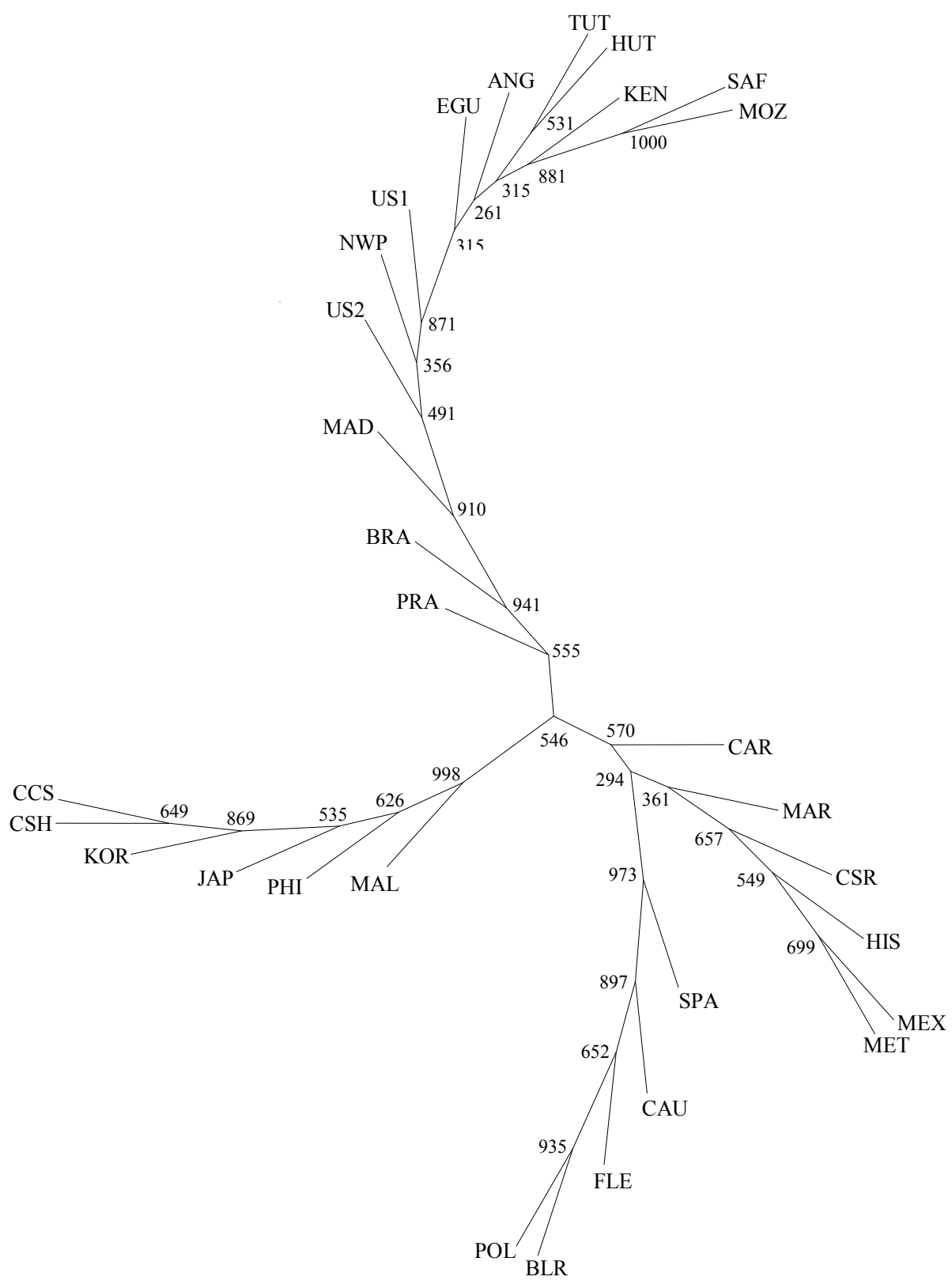




\begin{tabular}{|c|c|c|c|c|}
\hline Population & Abbreviation & $\begin{array}{c}\text { \# alleles } \\
\text { present }\end{array}$ & $\begin{array}{c}\text { Avg. } \\
\text { Heterozygosity }\end{array}$ & Reference \\
\hline \multicolumn{5}{|l|}{ AFRO-AMERICAN } \\
\hline US African American 1 (Minnesota) & US1 & 162 & 0.80380 & Gross and Budowle, 2006 \\
\hline US African American 2 (General) & US2 & 168 & 0.79573 & Butler et al., 2003 \\
\hline New Providence (Bahamas) & NWP & 173 & 0.78312 & present study \\
\hline \multicolumn{5}{|l|}{ AFRICAN } \\
\hline Cabinda (Angola) & ANG & 151 & 0.79698 & Beleza et al., 2004 \\
\hline Cape Town (South Africa) & SAF & 144 & - & Kido et al., 2007 \\
\hline Equatorial Guinea & EGU & 153 & 0.89193 & Alves et al., 2005 \\
\hline Hutu (Rwanda) & HUT & 154 & 0.81193 & Shepard and Herrera, 2006 \\
\hline Kenya & KEN & 139 & 0.81436 & Shepard and Herrera, 2006 \\
\hline Madagascar & MAD & 129 & 0.80350 & Regueiro et al., 2007 \\
\hline Maputo (Mozambique) & MOZ & 148 & 0.79580 & Alves et al., 2004 \\
\hline Tutsi (Rwanda) & TUT & 143 & 0.77818 & Regueiro et al., 2004 \\
\hline \multicolumn{5}{|l|}{ ASIAN } \\
\hline Chao Shan (South China) & $\mathrm{CCS}$ & 147 & 0.77793 & Hu et al., 2005 \\
\hline Japan & JAP & 152 & 0.77127 & Hashiyada et al., 2003 \\
\hline Korea & KOR & 140 & 0.77087 & Kim et al., 2003 \\
\hline Malaysia & MAL & 156 & 0.78127 & Seah et al., 2003 \\
\hline Philippines & PHI & 129 & 0.77793 & De Ungria et al., 2005 \\
\hline Shaanxi Han (NW China) & $\mathrm{CSH}$ & 160 & 0.78232 & Wang et al., 2005 \\
\hline \multicolumn{5}{|l|}{ LATIN AMERICAN } \\
\hline Caracas (Central Venezuela) & CAR & 161 & 0.79267 & Chiurillo et al., 2003 \\
\hline Central Mexico & MEX & 177 & 0.82827 & Hernández-Gutiérrez et al., 2005 \\
\hline Costa Rica & CSR & 190 & 0.85353 & Rodríguez et al., 2007 \\
\hline Hispanic (US -Minnesota) & HIS & 143 & 0.80120 & Gross and Budowle, 2006 \\
\hline Maracaibo (Venezuela) & MAR & 148 & 0.77896 & Bernal et al., 2006 \\
\hline Metztitlán (Mexico) & MET & 138 & 0.75067 & Gorostiza et al., 2007 \\
\hline Puerto Rico American (Massachusetts) & PRA & 149 & 0.79113 & Zúñiga et al., 2006 \\
\hline Rio de Janeiro (Brazil) & BRA & 161 & 0.85533 & de Souza Góes et al., 2004 \\
\hline \multicolumn{5}{|l|}{ EUROPEAN } \\
\hline Andalusia (South Spain) & SPA & 129 & 0.77600 & Coudray et al., 2007 \\
\hline Belaruse & BLR & 142 & 0.77160 & Rebala et al., 2007 \\
\hline Caucasian (US - General) & CAU & 153 & 0.78173 & Butler et al., 2003 \\
\hline Flemish (North Belgium) & FLE & 147 & 0.79533 & Mertens et al., 2006 \\
\hline North and Central Poland & POL & 155 & 0.79447 & Czarny et al., 2005 \\
\hline
\end{tabular}

Avg. Heterozygosity is not included for South Africa since observed heterozygositiy $\left(\mathrm{H}_{0}\right)$ values were not included by the authors

Table 1: Populations Analyzed 


\begin{tabular}{cccc}
\hline & \multicolumn{3}{c}{ Hybrid Population } \\
\hline Parental Groups & NWP & US1 & US2 \\
\hline West Africans & $0.468 \pm 0.048$ & $0.561 \pm 0.051$ & $0.485 \pm 0.043$ \\
East Africans & $0.368 \pm 0.050$ & $0.280 \pm 0.053$ & $0.353 \pm 0.045$ \\
Asian & $0.000 \pm 0.018$ & $0.000 \pm 0.019$ & $0.017 \pm 0.017$ \\
European & $0.163 \pm 0.020$ & $0.159 \pm 0.021$ & $0.146 \pm 0.018$ \\
\hline
\end{tabular}

Refer to Table 1 for key to abbreviations

Table 2: Admixture analysis for New Providence and two African American groups from the US

\begin{tabular}{ccccccccc}
\hline \multicolumn{7}{c}{ Hybrid Population } \\
\hline $\begin{array}{c}\text { Parental } \\
\text { Groups }\end{array}$ & BRA & PRA & CAR & HIS & CSR & MAR & MEX \\
\hline African & $0.410 \pm 0.016$ & $0.344 \pm 0.026$ & $0.273 \pm 0.026$ & $0.206 \pm 0.032$ & $0.190 \pm 0.035$ & $0.187 \pm 0.027$ & $0.152 \pm 0.037$ & $0.103 \pm 0.061$ \\
Asian & $0.037 \pm 0.016$ & $0.170 \pm 0.025$ & $0.175 \pm 0.025$ & $0.281 \pm 0.031$ & $0.235 \pm 0.034$ & $0.166 \pm 0.026$ & $0.285 \pm 0.036$ & $0.412 \pm 0.059$ \\
European & $0.553 \pm 0.018$ & $0.486 \pm 0.027$ & $0.551 \pm 0.028$ & $0.513 \pm 0.035$ & $0.575 \pm 0.037$ & $0.647 \pm 0.028$ & $0.563 \pm 0.040$ & $0.484 \pm 0.066$ \\
\hline
\end{tabular}

Refer to Table 1 for key to abbreviations

Table 3: Admixture analysis of Latin American populations 


\begin{tabular}{cccccccccc}
\hline & & & & Average $\boldsymbol{F}_{\text {st }}$ Distances \\
\hline Locus & Informative For & AF & AS & LA & EU & AF/AS & AF/LA & AF/EU \\
\hline D8S1179 & African and European Ancestry & 0.00152 & 0.00392 & 0.00155 & 0.00203 & 0.02396 & 0.01928 & 0.02894 \\
D21S11 & European \& Asian and African \& Asian populations & -0.00124 & 0.00546 & 0.00015 & -0.00100 & 0.04097 & 0.02397 & 0.00949 \\
D7S820 & Asian Ancestry & 0.00036 & 0.00622 & 0.00411 & 0.00060 & 0.05352 & 0.01855 & 0.00823 \\
CSF1PO & Not informative on the basis of ethnicity & 0.00604 & 0.00506 & -0.00060 & 0.00242 & 0.02016 & 0.01455 & 0.01338 \\
D3S1358 & Asian and European populations & 0.00041 & 0.00893 & 0.00937 & 0.00017 & 0.00525 & 0.00902 & 0.01601 \\
TH01 & African from Asian, Latin American and European populations & 0.00247 & 0.01177 & 0.01214 & 0.01128 & 0.07956 & 0.04914 & 0.09368 \\
D13S317 & African, Asian and European ancestry & -0.00282 & 0.00236 & 0.00790 & 0.00126 & 0.09371 & 0.03024 & 0.02383 \\
D16S539 & European ancestry & -0.00042 & 0.00603 & 0.00421 & 0.00118 & 0.00744 & 0.00919 & 0.02252 \\
D2S1338 & African and European Ancestry & -0.00016 & 0.00299 & 0.00580 & 0.00133 & 0.01820 & 0.01565 & 0.02243 \\
D19S433 & African and European Ancestry & -0.00064 & 0.01127 & 0.00438 & 0.00090 & 0.02070 & 0.01496 & 0.02527 \\
vWA & Not informative on the basis of ethnicity & -0.00129 & 0.01089 & 0.00416 & 0.00128 & 0.04757 & 0.01166 & 0.01457 \\
TPOX & African Ancestry & 0.00360 & 0.00116 & 0.00638 & 0.00421 & 0.05177 & 0.04658 & 0.07029 \\
D18S51 & African Ancestry & -0.00118 & 0.00349 & -0.00047 & 0.00058 & 0.03470 & 0.01635 & 0.01824 \\
D5S818 & African and European Ancestry & -0.00379 & 0.00398 & 0.00935 & 0.00061 & 0.04009 & 0.03141 & 0.01698 \\
FGA & European ancestry & -0.00117 & 0.00026 & 0.00261 & 0.00090 & 0.00415 & 0.00268 & 0.01199 \\
\hline
\end{tabular}

$\mathbf{A F}=$ African, $\mathbf{A S}=$ Asian, $\mathbf{L A}=$ Latin American and EU $=$ European

Table 4: Ancestry Informative Markers (AIMs) 


\begin{tabular}{|c|c|c|c|c|c|c|c|c|c|c|c|c|c|c|c|c|c|c|c|c|c|c|c|c|c|c|c|c|c|c|c|c|}
\hline \multirow{2}{*}{ Sample \# } & \multicolumn{2}{|c|}{ D8S1179 } & \multicolumn{2}{|c|}{ D21S11 } & \multicolumn{2}{|c|}{ D7S820 } & \multicolumn{2}{|c|}{ CSF1PO } & \multicolumn{2}{|c|}{ D3S1358 } & \multicolumn{2}{|c|}{ TH01 } & \multicolumn{2}{|c|}{ D13S317 } & \multicolumn{2}{|c|}{ D16S539 } & \multicolumn{2}{|c|}{ D2S1338 } & \multicolumn{2}{|c|}{ D19S433 } & \multicolumn{2}{|c|}{ vWA } & TPO & & D18 & S51 & $\mathbf{A M}$ & & D5S & $\mathbf{S 8 1 8}$ & FC & \\
\hline & 1 & 2 & 1 & 2 & 1 & 2 & 1 & 2 & 1 & 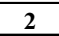 & 1 & 2 & 1 & 2 & 1 & 2 & 1 & 2 & 1 & 2 & 1 & 2 & 1 & 2 & 1 & 2 & 1 & 2 & 1 & 2 & 1 & 2 \\
\hline Nwp001 & 14 & 18 & 31.2 & 33.2 & 10 & 10 & 7 & 11 & 15 & 17 & 6 & 7 & 13 & 13 & 10 & 12 & 19 & 22 & 12 & 14 & 16 & 19 & 8 & 9 & 13 & 17 & $\mathrm{X}$ & $\mathrm{Y}$ & 11 & 13 & 20 & 25 \\
\hline Nwp002 & 13 & 14 & 30 & 31.2 & 8 & 8 & 12 & 12 & 14 & 18 & 7 & 7 & 12 & 12 & 11 & 12 & 17 & 17 & 14 & 14.2 & 16 & 17 & 10 & 11 & 16 & 16 & $\mathrm{X}$ & $\mathrm{Y}$ & 11 & 13 & 21 & 25 \\
\hline Nwp003 & 14 & 14 & 34 & 37 & 10 & 10 & 10 & 12 & 15 & 16 & 8 & 8 & 11 & 13 & 12 & 12 & 21 & 23 & 12 & 13.2 & 17 & 18 & 11 & 11 & 13 & 15 & $\mathrm{X}$ & $\mathrm{Y}$ & 11 & 12 & 23 & 31.2 \\
\hline Nwp004 & 13 & 15 & 29 & 30 & 10 & 10 & 11 & 12 & 15 & 15 & 7 & 8 & 12 & 12 & 11 & 13 & 20 & 22 & 14.2 & 15 & 16 & 17 & & 11 & 16 & 21 & $\mathrm{X}$ & $\mathrm{Y}$ & 13 & 13 & 22 & 26 \\
\hline Nwp005 & 13 & 14 & 29 & 29 & 7 & 8 & 11 & 12 & 16 & 18 & 8 & 9.3 & 13 & 14 & 12 & 13 & 17 & 20 & 11 & 13 & 16 & 18 & 8 & 8 & 12 & 16 & $\mathrm{X}$ & $\mathrm{Y}$ & 12 & 12 & 21 & 22 \\
\hline Nwp006 & 14 & 15 & 27 & 30 & 8 & 10 & 10 & 11 & 15 & 16 & 7 & 7 & 11 & 14 & 10 & 13 & 19 & 21 & 1 & 14.2 & 15 & 17 & 9 & 10 & 15 & 18 & $\mathrm{X}$ & $\mathrm{Y}$ & 11 & 12 & 18.2 & 21 \\
\hline Nwp007 & 12 & 13 & 29 & 31.2 & 10 & 11 & 11 & 12 & 16 & 17 & 8 & 9 & 11 & 13 & 10 & 12 & 17 & 18 & 13 & 14 & 16 & 16 & 11 & 12 & 14 & 14 & $\mathrm{X}$ & $\mathrm{Y}$ & 11 & 11 & 19 & 27 \\
\hline Nwp008 & 11 & 14 & 30 & 32.2 & 11 & 11 & 7 & 10 & 15 & 17 & 6 & 6 & 11 & 12 & 11 & & 19 & 22 & 1 & 16.2 & 15 & 17 & & 8 & 15 & 17 & $X$ & $\mathrm{X}$ & 11 & 12 & 22 & 23 \\
\hline Nwp009 & 13 & 16 & 28 & 32.2 & 10 & 12 & 11 & 12 & 16 & 17 & 7 & 7 & 12 & 13 & 9 & 9 & 17 & 23 & 13 & 14 & 15 & 18 & 6 & 10 & 18 & 18 & $\mathrm{X}$ & $\mathrm{Y}$ & 11 & 12 & 24 & 24 \\
\hline Nwp010 & 11 & 14 & 28 & 28 & 8 & 9 & 9 & 12 & 14 & 18 & 6 & 9.3 & 10 & 11 & 11 & 14 & 20 & 21 & 1 & 13 & 15 & 17 & 9 & 10 & 15 & 22 & $\mathrm{X}$ & $\mathrm{Y}$ & 11 & 12 & 20 & 21 \\
\hline Nwp011 & 10 & 14 & 27 & 35 & 8 & 11 & 7 & & 15 & 16 & 7 & 7 & 12 & 13 & 9 & 12 & 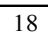 & 20 & 1 & 14.2 & 16 & 18 & 11 & 11 & 17 & 17 & $\mathrm{X}$ & $\mathrm{Y}$ & 8 & 12 & 23 & 23 \\
\hline Nwp012 & 12 & 16 & 29 & 31.2 & 10 & 13 & 7 & 7 & 15 & 16 & 9 & 9.3 & 12 & 13 & 11 & & & 18 & 1 & 13 & 15 & 18 & 6 & 10 & 16 & 20 & $\mathrm{X}$ & $\mathrm{Y}$ & 11 & 12 & 2 & 23 \\
\hline Nwp013 & 14 & 14 & 28 & 29 & 9 & 11 & 12 & 12 & 14 & 17 & 7 & 8 & 12 & 13 & 11 & 11 & 1 & 23 & 1 & & 16 & 18 & 9 & 9 & 1 & 14 & $\mathrm{X}$ & $\mathrm{Y}$ & 8 & 12 & 23 & 26 \\
\hline Nwp014 & 14 & 14 & 31 & 33.2 & 10 & 12 & 10 & 12 & 15 & 16 & 6 & 8 & 12 & 13 & 10 & 12 & 2. & 22 & 1 & 1 & 18 & 19 & 9 & 9 & 12 & 18 & $\mathrm{X}$ & $\mathrm{Y}$ & 12 & 13 & 21 & 24 \\
\hline Nwp015 & 13 & 14 & 29 & 29 & 9 & 11 & 11 & 12 & 17 & 18 & 6 & & & 1 & 11 & & 2 & 2 & 1 & & 15 & 18 & 9 & 11 & 1 & 19 & $\mathrm{X}$ & $\mathrm{Y}$ & 13 & & 22 & 23 \\
\hline Nwp016 & 13 & 15 & 30 & 35 & 8 & 9 & 11 & 12 & 16 & 17 & 8 & 8 & 12 & 1 & 11 & 1 & & 2 & 1 & & 5 & 19 & 8 & 9 & 1 & & $\mathrm{X}$ & $\mathrm{Y}$ & 13 & 3 & 9 & 24 \\
\hline Nwp017 & 13 & 14 & 29 & 29 & 9 & 11 & 11 & 12 & 17 & 18 & 6 & 8 & 8 & 12 & 11 & 12 & 22 & 23 & 13 & 15 & 15 & 18 & 9 & 11 & 1. & 19 & $\mathrm{X}$ & $\mathrm{Y}$ & 13 & 13 & 22 & 23 \\
\hline Nwp018 & 12 & 12 & 30 & 30 & 8 & 12 & 8 & 10 & 15 & 17 & 7 & 7 & 12 & 12 & 12 & 12 & 18 & 23 & 15.2 & 15.2 & 15 & 15 & 9 & 11 & 16 & 17 & $\mathrm{X}$ & $\mathrm{Y}$ & 11 & 13 & 22 & 23 \\
\hline Nwp019 & 11 & 14 & 29 & 32.2 & 8 & 11 & 10 & 11 & 12 & 18 & 7 & 9 & 12 & 13 & 11 & 1 & 16 & 23 & \begin{tabular}{l|l|}
9 \\
\end{tabular} & 13.2 & 16 & 18 & 9 & 9 & 13 & 16 & $\mathrm{X}$ & $\mathrm{X}$ & 10 & 13 & 25 & 26 \\
\hline & 13 & 15 & 30 & 35 & 10 & 11 & 7 & 12 & 15 & 16 & 7 & 8 & 10 & 11 & 13 & 1 & & 16 & 14 & 15 & 15 & 15 & 8 & 9 & & 1 & $\mathrm{X}$ & $\mathrm{Y}$ & & 2 & 23 & 24 \\
\hline Nwp021 & 14 & 15 & 28 & 28 & 10 & 13 & 10 & 12 & 16 & 17 & 7 & 9.3 & 12 & 13 & 10 & 1 & 21 & 25 & 12.2 & 12.2 & 14 & 18 & 8 & 9 & 12 & 18 & $\mathrm{X}$ & Y & 8 & 12 & 19 & 23 \\
\hline Nwp022 & 12 & 14 & 30 & 35 & 8 & 10 & 8 & 10 & 16 & 17 & 6 & 7 & 1. & 1. & 11 & 1 & 15 & 19 & 11 & 14 & 15 & 19 & 8 & 11 & 1 & 17 & $\mathrm{X}$ & $\mathrm{X}$ & 11 & 12 & 22 & 23 \\
\hline Nwp023 & 13 & 15 & 30 & 3 & 9 & 11 & 8 & 10 & 15 & 16 & 6 & 9.3 & 12 & 13 & 11 & 12 & 16 & 27 & 12.2 & 13.2 & 15 & 15 & 6 & 9 & 13 & 18 & $\mathrm{x}$ & $\mathrm{Y}$ & 11 & 13 & 23 & 24 \\
\hline & 13 & 15 & 30 & 3 & 12 & 12 & 8 & 8 & 14 & 15 & 7 & 9 & 11 & 1 & 9 & 10 & 1 & 19 & 11.2 & 13 & 15 & 16 & 8 & 11 & 1 & 19 & $\mathrm{X}$ & $\mathrm{Y}$ & 12 & 3 & 24 & 25 \\
\hline Nwp025 & 15 & 15 & 30 & 30 & 9 & 10 & 10 & 12 & 14 & 17 & 6 & 7 & 11 & 1 & 10 & & 2 & 24 & 13 & 14.2 & 16 & 18 & 8 & 9 & 1 & 20 & $\mathrm{X}$ & $\mathrm{Y}$ & 12 & 12 & 22 & 23 \\
\hline Nwp026 & 10 & 15 & 28 & 30 & 10 & 11 & 10 & 11 & 16 & 18 & 9 & 9 & 14 & 14 & 13 & 1 & 19 & $1 \mathrm{~s}$ & 1 & 11 & 14 & 17 & 7 & 9 & 19 & 20 & $\mathrm{X}$ & $\mathrm{Y}$ & 11 & 11 & 21 & 29 \\
\hline Nwp027 & 13 & 16 & 28 & 30 & 10 & 10 & 9 & 13 & 15 & 16 & 8 & 9 & 11 & 11 & 11 & 12 & 21 & 22 & 1 & 1 & 16 & 16 & 8 & 9 & 1 & 17 & $\mathrm{X}$ & $\mathrm{Y}$ & 12 & 13 & 23 & 25 \\
\hline & 11 & 13 & 28 & 30.2 & 10 & 11 & 7 & 12 & 15 & 16 & 7 & 8 & 12 & & 11 & & 15 & & & & 15 & 15 & 10 & 10 & 1 & 1 & $\mathrm{X}$ & $\mathrm{Y}$ & 12 & 13 & 19 & 23 \\
\hline Nwp029 & 12 & 15 & 28 & 28 & 8 & 10 & 11 & 13 & 15 & 15.2 & 7 & 7 & 11 & 12 & 9 & 12 & 19 & 22 & 1 & 1 & 17 & 18 & 8 & 11 & 16 & 20 & $\mathrm{X}$ & $\mathrm{Y}$ & 8 & 11 & 24 & 25 \\
\hline Nwp030 & 14 & 15 & 28 & 30 & 10 & 11 & 10 & 11 & 15 & 16 & 7 & 9 & 11 & 12 & 9 & 11 & 17 & 21 & 1 & 15.2 & 15 & 19 & 9 & 11 & 14 & 15 & $\mathrm{X}$ & $\mathrm{Y}$ & 12 & 12 & 20 & 21 \\
\hline & 11 & 12 & 30 & 30 & 9 & 10 & 10 & 11 & 16 & 17 & 7 & 9 & 12 & 14 & 11 & 12 & 17 & 24 & 1 & 15.2 & 15 & 16 & 9 & 9 & 12 & 15 & $\mathrm{X}$ & $\mathrm{Y}$ & 12 & 12 & 23 & 25 \\
\hline Nwp032 & 14 & 1 & 28 & 3 & 8 & 8 & 10 & 12 & 15 & 1 & 7 & 9.3 & 10 & 1 & 9 & 1 & 2 & 2 & & & 16 & 17 & 8 & 9 & & & $\mathrm{X}$ & $\mathrm{Y}$ & 11 & 12 & 22 & 24 \\
\hline Nwp033 & 11 & 13 & 30 & 31 & 8 & 8 & 10 & 10 & 15 & 18 & 6 & 7 & 12 & 12 & 11 & 12 & 18 & 21 & 13 & & 15 & 15 & 8 & 9 & 14 & 22 & $\mathrm{X}$ & $\mathrm{Y}$ & 10 & 13 & 22 & 23 \\
\hline Nwp034 & 12 & 12 & 31 & 34 & 10 & 13 & 12 & 12 & 14 & 15 & 6 & 7 & 12 & 12 & 6 & 11 & 22 & 24 & 1. & 15.2 & 13 & 15 & 11 & 11 & 15 & 16 & $\mathrm{X}$ & $\mathrm{Y}$ & 8 & 11 & 22 & 27 \\
\hline & 14 & 14 & 31 & 31 & 8 & 9 & 10 & 11 & 15 & & 6 & & 11 & 12 & 12 & & 17 & & & & 14 & 16 & 10 & 11 & 1 & 17 & $\mathrm{X}$ & $\mathrm{Y}$ & 13 & 14 & 20 & 21 \\
\hline Nwp036 & 13 & 14 & 28 & 28 & 8 & 10 & 11 & 11 & 15 & 17 & 7 & 8 & 11 & 12 & 11 & 12 & 21 & 22 & 1 & 13.2 & 17 & 17 & 8 & 11 & 19 & 20 & $\mathrm{X}$ & $\mathrm{Y}$ & 12 & 13 & 22 & 25 \\
\hline Nwp037 & 12 & 14 & 30 & 34 & 10 & 11 & 8 & 8 & 16 & 17 & 7 & 8 & 12 & 14 & 9 & 11 & 17 & 21 & 13 & 13 & 15 & 16 & 10 & 11 & 14 & 19 & $\mathrm{X}$ & $\mathrm{Y}$ & 13 & 15 & 22 & 23 \\
\hline Nwp038 & 15 & 17 & 29 & 30 & 10 & 12 & 9 & 9 & 14 & 15 & 7 & $\varepsilon$ & 13 & 13 & $\mathrm{c}$ & & 16 & 2 & 1 & 1 & 17 & 17 & 8 & 10 & 17 & 18 & $\mathrm{X}$ & $X$ & 12 & 12 & 24 & 26 \\
\hline Nwp039 & 11 & 14 & 28 & 30 & 8 & 12 & 11 & 11 & 1 & 16 & 7 & 9 & 9 & 11 & 10 & 13 & 18 & 18 & 13 & & 16 & 18 & 9 & 11 & 16 & 16 & $\mathrm{X}$ & $Y$ & 8 & 13 & 34.2 & 34.2 \\
\hline Nwp040 & 13 & 15 & 27 & 29 & 11 & 11 & 9 & 10 & 14 & 15 & 7 & 8 & 11 & 14 & 11 & 11 & 19 & 22 & 12 & 13 & 13 & 14 & 8 & 10 & 16 & 16 & $\mathrm{X}$ & $\mathrm{Y}$ & 8 & 13 & 21 & 24 \\
\hline
\end{tabular}

\section{Supplementary Table 1: Genotype Assignment}




\begin{tabular}{|c|c|c|c|c|c|c|c|c|c|c|c|c|c|c|c|c|c|c|c|c|c|c|c|c|c|c|c|c|c|c|c|c|}
\hline le \# & 85 & 1179 & D21 & S11 & D7s & 820 & CSF & PO & D3S & 1358 & TH & & D13S & 317 & D16 & 539 & D2S & 338 & D19S & 4433 & vW & & TPC & & D18 & S51 & $\mathbf{A M}$ & IEL & D5S & 818 & FG & \\
\hline & 1 & 2 & 1 & 2 & 1 & 2 & 1 & 2 & 1 & 2 & 1 & 2 & 1 & 2 & 1 & 2 & 1 & 2 & 1 & 2 & \begin{tabular}{l|l}
1 & \\
\end{tabular} & 2 & \begin{tabular}{l|l}
1 \\
\end{tabular} & 2 & 1 & \begin{tabular}{|l|}
2 \\
\end{tabular} & 1 & 2 & 1 & 2 & 1 & 2 \\
\hline Nwp041 & 13 & 15 & 28 & 29 & 10 & 12 & 7 & 12 & 17 & 17 & 8 & 8 & 11 & 12 & 10 & 13 & 20 & 23 & 13 & 14.2 & 17 & 18 & 8 & 11 & 12 & 15 & $\mathrm{X}$ & $\mathrm{X}$ & & 12 & 25 & 27 \\
\hline Nwp042 & & & 27 & 32 & & & & & 16 & 16 & 7 & & 11 & & & 14 & & 23 & 12.2 & 13.2 & 15 & 16 & 8 & 11 & 18 & 8 & $\mathrm{X}$ & $\mathrm{X}$ & & 13 & 21 & 23 \\
\hline Nwp043 & & & 29 & 34 & & 9 & & & 15 & 16 & & 9 & 14 & & 10 & 10 & & 25 & 12 & 14 & 15 & 17 & 8 & 12 & 12 & 17 & $\mathrm{X}$ & & 11 & 12 & 21 & 23 \\
\hline Nwp044 & & & 30 & 30 & & 10 & & & 15 & 16 & & 8 & 8 & & 10 & 10 & & & 13 & & 14 & 14 & 8 & 11 & 6 & & $\mathrm{X}$ & $\mathrm{Y}$ & 12 & 13 & 21 & 23 \\
\hline & & & 29 & 29 & & 10 & & & 14 & 17 & & & 12 & & & 11 & & & & & 16 & 19 & & & 5 & 8 & $X$ & & 11 & 13 & 23 & 27 \\
\hline & & & 29 & 31.2 & 8 & 11 & & & 16 & 17 & & 7 & 1 & & & 1 & & & 13 & & 15 & 20 & & 11 & 5 & & $\mathrm{X}$ & & 3 & 13 & 22 & 24 \\
\hline & & & 38 & 32 & 10 & 1 & & & 15 & 1 & 7 & 8 & 11 & & 11 & 12 & & & 13.2 & & 3 & 16 & 0 & 11 & 5 & & $\mathrm{X}$ & $\mathrm{X}$ & 2 & 12 & 1 & 27 \\
\hline & & & 29 & 32 & & 11 & & & 14 & & & & & & & 13 & & & 12 & & 16 & 16 & 10 & 11 & 5 & & $\mathrm{X}$ & & 2 & 13 & 22 & 24 \\
\hline & & & 2 & & 9 & 10 & & & 15 & 1 & & & & & & & & & & & 13 & 18 & & & & & & & 11 & & 22 & 22 \\
\hline & & & 2 & 33.2 & 10 & & & & 1 & 1 & 8 & & 1 & & & 1 & & & & & 14 & 16 & & 10 & & & $\mathrm{X}$ & & 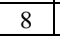 & 13 & 23 & 24 \\
\hline & & & 28 & 30 & & & & & 16 & & 6 & & 12 & & 11 & & & & & & 17 & 17 & & 10 & & & $\mathrm{X}$ & & & 11 & 24 & 25 \\
\hline & & & 30 & 30.2 & 1 & 12 & & & 1 & 1 & & c & & & & 1 & & & & & 16 & 17 & & & & & & & & & 20 & 27 \\
\hline & & & 31 & 35 & 8 & & & & 1 & 1 & 7 & 7 & 1 & & & & & & & & 5 & 17 & & & 8 & & $\mathrm{X}$ & & 2 & 12 & 19 & 22 \\
\hline & & 1 & 32.2 & 32.2 & 9 & 12 & & & 1 & & 8 & 9 & 1 & & & 1 & & & & & & 17 & & 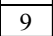 & & & $\mathrm{K}$ & & 1 & 3 & 16.1 & 23 \\
\hline & & & 28 & 28 & & & & & 1 & & 7 & 7 & & & & & & & & & 6 & 18 & 11 & 11 & & & $\mathrm{X}$ & & 10 & 12 & 19 & 25 \\
\hline & & & 3 & & & & & & & & & & & & & & & & 13 & & & 1 & & & & & & & & & & 22 \\
\hline & & 1 & 2 & 2 & 1 & & & 1 & & & 7 & 7 & 1 & & & 1 & & & & & 1 & 1 & & & & & $\mathrm{~K}$ & & 1 & 2 & & 22 \\
\hline & & & & & & & & & & & 6 & & & & & & & & & & 14 & 19 & 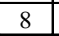 & & & & $\mathrm{X}$ & $\bar{Y}$ & 12 & 13 & & 29 \\
\hline & & & 30 & & & 18 & & & 17 & 1 & & & & & & & & & 1 & 15. & 17 & 19 & & & 14 & 1 & $X$ & & 8 & 13 & 22 & 22 \\
\hline & & & & & & & & & & & & 9.3 & 1 & & & & & & & & 5 & 17 & 0 & 11 & & & $\mathrm{x}$ & & 1 & & & 22 \\
\hline & & & & 3 & 8 & & & & 1 & & 7 & 7 & & & & & & & & & & 18 & & & & & $\mathrm{X}$ & & 0 & & & 22 \\
\hline & & & 28 & 31.2 & 10 & 1 & & & 14 & & 7 & & & & & & & & & & 16 & 17 & & & & & $\mathrm{X}$ & & 12 & 12 & 3 & 24 \\
\hline & & 1 & & 3 & & & & & 1 & & 8 & $\begin{array}{l}9.3 \\
\end{array}$ & & & & 1 & & & & & 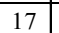 & 19 & 11 & 11 & & & $\mathrm{X}$ & & 10 & 13 & 21 & 24 \\
\hline & & 1 & 2 & 2 & 8 & 8 & 7 & & 1 & & 6 & 7 & 1 & & & 1 & & & 1 & & & 15 & 10 & 11 & 2 & & $\mathrm{X}$ & $\mathrm{X}$ & 1 & 12 & 23 & 27 \\
\hline & & 1 & & & & 11 & & & 1 & & 7 & 9.3 & 1 & & & & & & & & & 18 & & & & & $\mathrm{X}$ & & 11 & 13 & & 28 \\
\hline & & & & & & & & & & & & & 11 & & & & & & 13 & & 16 & 20 & & & & & $\mathrm{X}$ & & 8 & 12 & 19 & 23 \\
\hline & 1 & 1 & 3 & & 1 & 1 & & & & & 9 & & 1 & & & 1 & & & 1 & & 1 & 16 & & 11 & & & $X$ & & 7 & 12 & 21 & 24 \\
\hline & & 1. & 2 & 31.2 & 1 & & 1 & & 1 & 1 & 7 & 9.3 & 1 & & & & & & & & 11 & 1 & & 11 & & & $\mathrm{X}$ & & 11 & 12 & 17.2 & 21 \\
\hline & & & & & & & & & & & & & & & & & & & & & 17 & 1 & & & & & $X$ & & & 11 & 22 & 22 \\
\hline & & 1 & 2 & 2 & & & & & & & 7 & & 1 & & & & & & & & 17 & 17 & 6 & & & & $\mathrm{X}$ & $\bar{Y}$ & 11 & 12 & 19 & 26 \\
\hline $\bar{N}$ & 1 & 1 & 28 & 2 & 9 & 10 & 11 & 11 & 15 & & 7 & 7 & 1 & & 9 & 1 & & 2 & & & 1 & 16 & 6 & 11 & 2 & & $\mathrm{X}$ & $\mathrm{X}$ & 10 & 13 & 24 & 27 \\
\hline & 1 & 15 & 31.2 & 3. & 11 & 12 & 11 & 1 & 16 & 1 & 8 & 9.3 & 12 & & & 11 & & 1 & & & 14 & 15 & 8 & 12 & & & $\mathrm{X}$ & & 10 & 10 & 22 & 24 \\
\hline & 1 & 14 & 27 & & & & & & & & & & & & & & & & & & & 20 & & & & & $X$ & & & & 22 & 24 \\
\hline & 1 & 16 & 28 & 3 & 1 & 1 & 1 & 11 & 1 & & 6.3 & 9 & 1 & & 9 & 1 & & 2 & & 15.2 & & 16 & 8 & 11 & & & $\mathrm{X}$ & $\mathrm{Y}$ & 8 & 10 & 25 & 26 \\
\hline & 1 & 15 & 28 & 3 & 1 & 11 & 10 & 11 & 15 & 15.2 & 8 & 9 & 12 & 14 & 10 & 10 & 21 & 23 & 1 & 1 & 16 & 16 & 8 & 8 & 15 & 16 & $\mathrm{X}$ & $\mathrm{Y}$ & 12 & 13 & 22 & 22 \\
\hline & 1 & 1 & 2 & & 10 & 11 & 11 & 1 & 1 & 16 & & & 13 & & & 12 & & & & & 14 & 16 & & & & & $\mathrm{X}$ & & 12 & 12 & 21 & 22 \\
\hline & 1 & 1 & 2 & & 8 & 8 & 11 & & 1 & & & & 11 & & & & & 1 & & & 15 & 1 & & & & & $\mathrm{X}$ & & 12 & & 3 & 23 \\
\hline & 14 & 15 & 28 & 28 & 8 & 10 & 11 & 12 & 16 & 1 & 7 & 7 & 11 & 12 & 1 & 1 & 21 & 2 & 13.2 & 1 & 16 & 17 & 8 & 11 & 17 & 9 & $X$ & $\mathrm{Y}$ & 11 & 12 & 23 & 24 \\
\hline Nwp079 & 13 & 13 & 32.2 & 32.2 & 9 & 12 & 11 & 12 & 16 & 17 & 7 & 8 & 11 & 12 & 11 & 13 & 19 & 23 & 13 & 13 & 15 & 16 & 6 & 9 & 19 & 22 & $X$ & $\mathrm{X}$ & 12 & 12 & 18.2 & 26 \\
\hline Nwp080 & 12 & 15 & 29 & 32.2 & 9 & 11 & 12 & 12 & 15 & 17 & 7 & 9 & 11 & 12 & 11 & 13 & 19 & 19 & 13 & 14 & 17 & 19 & 10 & 11 & 13 & 15 & $\mathrm{X}$ & $\mathrm{X}$ & 12 & 12 & 24 & 25 \\
\hline
\end{tabular}

Supplementary Table 1: Genotype Assignment (Cont'd) 


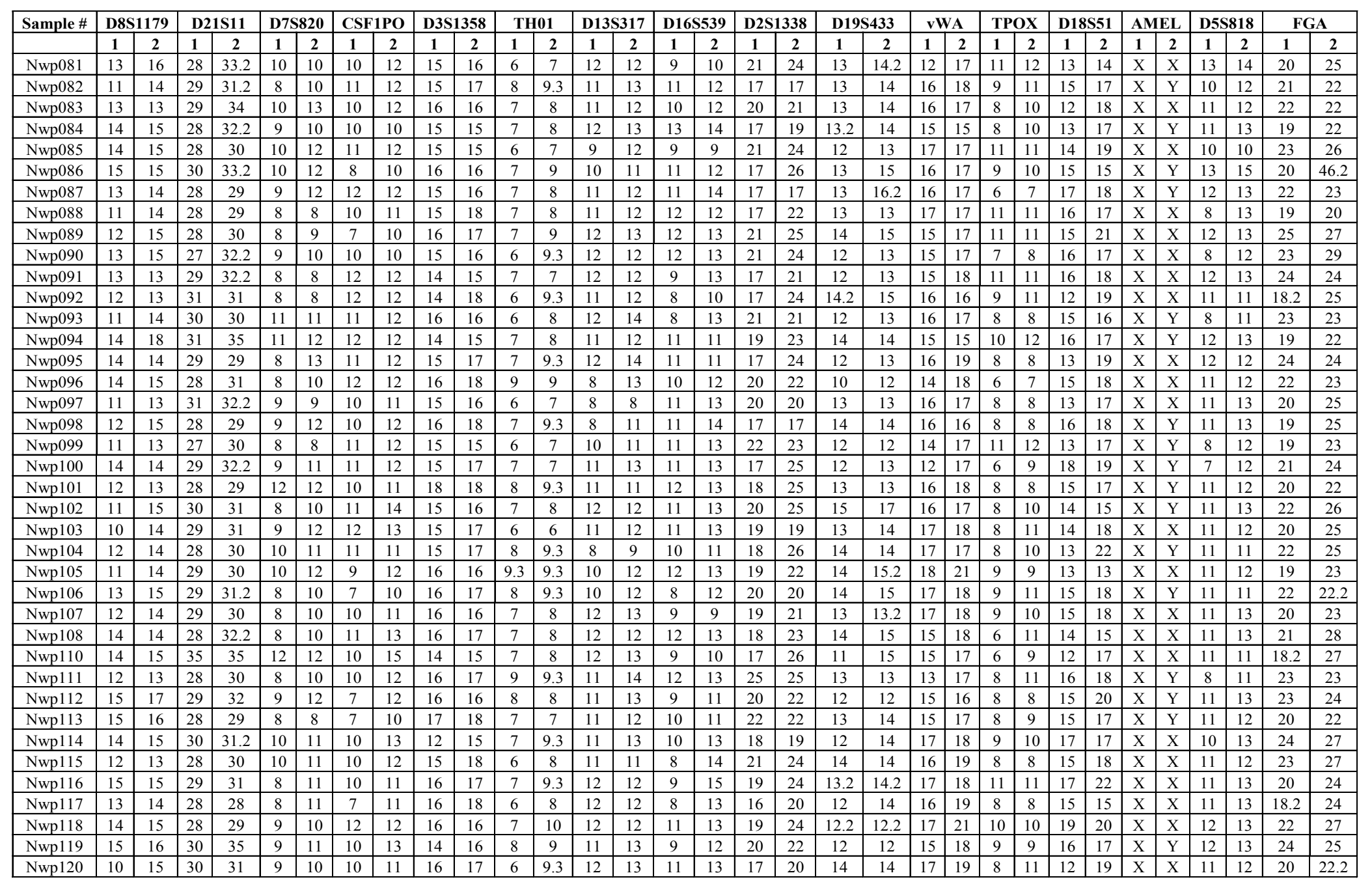

Supplementary Table 1: Genotype Assignments (Cont'd) 


\begin{tabular}{|c|c|c|c|c|c|c|c|c|c|c|c|c|c|c|c|c|c|c|c|c|c|c|c|c|c|c|c|c|c|c|c|c|}
\hline \multirow[t]{2}{*}{ Sample \# } & \multicolumn{2}{|c|}{ D8S1179 } & \multicolumn{2}{|c|}{ D21S11 } & \multicolumn{2}{|c|}{ D7S820 } & \multicolumn{2}{|c|}{ CSF1PO } & \multicolumn{2}{|c|}{ D3S1358 } & \multicolumn{2}{|c|}{ TH01 } & \multicolumn{2}{|c|}{ D13S317 } & \multicolumn{2}{|c|}{ D16S539 } & \multicolumn{2}{|c|}{ D2S1338 } & \multicolumn{2}{|c|}{ D19S433 } & \multicolumn{2}{|c|}{ vWA } & $\mathbf{T P}$ & OX & D18: & S51 & $\mathbf{A M}$ & & D5S & 818 & FG & $\mathrm{BA}$ \\
\hline & 1 & 2 & 1 & 2 & 1 & 2 & 1 & 2 & 1 & 2 & 1 & 2 & 1 & 2 & 1 & 2 & 1 & 2 & 1 & 2 & 1 & 2 & 1 & 2 & 1 & 2 & 1 & 2 & 1 & 2 & 1 & 2 \\
\hline Nwp121 & 11 & 14 & 27 & 28 & 10 & 10 & 7 & 10 & 17 & 17 & 6 & 7 & 12 & 13 & 12 & 14 & 20 & 22 & 12 & 13 & 14 & 17 & 9 & 11 & 13 & 13 & $\mathrm{X}$ & $\mathrm{X}$ & 13 & 13 & 21 & 22 \\
\hline Nwp122 & 11 & 17 & 29 & 32 & 8 & 10 & 10 & 11 & 14 & 16 & 8 & 8 & 11 & 12 & 9 & 11 & 16 & 18 & 13 & 13 & 16 & 17 & 8 & 9 & 14 & 16 & $\mathrm{X}$ & $\mathrm{x}$ & 12 & 13 & 22 & 23 \\
\hline Nwp123 & 14 & 15 & 29 & 30 & 9 & 11 & 10 & 12 & 16 & 17 & 8 & 9 & 10 & 11 & 10 & 12 & 21 & 23 & 13 & 14.1 & 15 & 16 & 9 & 10 & 16 & 17 & $\mathrm{X}$ & $\mathrm{X}$ & 11 & 12 & 23 & 26 \\
\hline Nwp124 & 12 & 16 & 28 & 31 & 9 & 10 & 7 & 11 & 15 & 18 & 6 & 7 & 11 & 11 & 9 & 9 & 17 & 22 & 13 & 15 & 16 & 19 & 8 & 12 & 17 & 18 & $\mathrm{X}$ & $\mathrm{X}$ & 12 & 13 & 24 & 24 \\
\hline Nwp125 & 11 & 13 & 28 & 28 & 9 & 10 & 7 & 11 & 15 & 16 & 8 & 8 & 11 & 14 & 10 & 11 & 17 & 19 & 12 & 13 & 16 & 17 & 6 & 11 & 12 & 15 & $\mathrm{X}$ & $\mathrm{Y}$ & 11 & 13 & 21 & 24 \\
\hline Nwp126 & 12 & 14 & 28 & 29 & 9 & 12 & 12 & 12 & 14 & 16 & 7 & 9.3 & 11 & 12 & 9 & 12 & 21 & 23 & 13 & 14 & 18 & 19 & 11 & 11 & 16 & 18 & $\mathrm{X}$ & $\mathrm{X}$ & 12 & 12 & 18.2 & 27 \\
\hline Nwp127 & 13 & 15 & 28 & 30 & 8 & 12 & 10 & 12 & 16 & 16 & 9 & 9.3 & 8 & 11 & 11 & 13 & 17 & 17 & 13 & 15 & 15 & 17 & 8 & 8 & 13 & 18 & $\mathrm{X}$ & $\mathrm{X}$ & 11 & 12 & 23 & 25 \\
\hline Nwp128 & 14 & 14 & 29 & 29 & 9 & 11 & 8 & 12 & 14 & 16 & 6 & 8 & 12 & 12 & 9 & 9 & 16 & 19 & 12 & 13 & 14 & 16 & 9 & 10 & 13 & 16 & $\mathrm{X}$ & $\mathrm{X}$ & 13 & 13 & 18.2 & 21 \\
\hline Nwp129 & 13 & 15 & 28 & 29 & 10 & 10 & 12 & 12 & 15 & 16 & 7 & 7 & 11 & 12 & 9 & 11 & 16 & 22 & 13 & 14 & 15 & 15 & 11 & 12 & 17 & 19 & $\mathrm{X}$ & $\mathrm{Y}$ & 11 & 11 & 23 & 26 \\
\hline Nwp130 & 8 & 15 & 28 & 29 & 10 & 10 & 11 & 12 & 16 & 16 & 6 & 6 & 11 & 12 & 10 & 13 & 22 & 23 & 10 & 13 & 17 & 18 & 8 & 8 & 18 & 21 & $\mathrm{X}$ & $\mathrm{Y}$ & 11 & 14 & 26 & 27 \\
\hline Nwp131 & 12 & 14 & 28 & 28 & 10 & 13 & 11 & 12 & 15 & 16 & 7 & 7 & 12 & 12 & 11 & 11 & 21 & 24 & 14 & 16.2 & 18 & 18 & 8 & 8 & 12 & 17 & $\mathrm{X}$ & $\mathrm{Y}$ & 10 & 13 & 22 & 25 \\
\hline Nwp132 & 14 & 15 & 29 & 34 & 10 & 11 & 10 & 10 & 17 & 17 & 7 & 9 & 11 & 13 & 9 & 11 & 17 & 22 & 14 & 15 & 15 & 16 & 8 & 11 & 15 & 19 & $\mathrm{X}$ & $\mathrm{X}$ & 11 & 13 & 23 & 25 \\
\hline Nwp133 & 12 & 15 & 29 & 29 & 8 & 9 & 10 & 11 & 15 & 16 & 8 & 9.3 & 12 & 12 & 10 & 11 & 17 & 26 & 13 & 14 & 15 & 16 & 8 & 9 & 16 & 18 & $\mathrm{X}$ & $\mathrm{Y}$ & 11 & 12 & 23 & 24 \\
\hline Nwp134 & 10 & 14 & 30 & 31 & 8 & 10 & 8 & 13 & 16 & 16 & 7 & 9 & 12 & 12 & 11 & 11 & 19 & 22 & 11 & 13 & 16 & 16 & 10 & 11 & 16 & 22 & $\mathrm{X}$ & $\mathrm{Y}$ & 12 & 13 & 24 & 28 \\
\hline Nwp135 & 11 & 15 & 32.2 & 32.2 & 8 & 10 & 8 & 8 & 16 & 17 & 6 & 7 & 12 & 13 & 9 & 13 & 20 & 21 & 12.2 & 13 & 14 & 16 & 9 & 10 & 16 & 16 & $\mathrm{X}$ & $\mathrm{X}$ & 11 & 13 & 19 & 21 \\
\hline Nwp136 & 12 & 13 & 28 & 30 & 8 & 10 & 12 & 13 & 16 & 16 & 7 & 7 & 11 & 12 & 11 & 12 & 17 & 18 & 12 & 14 & 16 & 18 & 9 & 12 & 17 & 18 & $\mathrm{X}$ & $\mathrm{X}$ & 11 & 13 & 23 & 24 \\
\hline Nwp137 & 13 & 14 & 27 & 29 & 11 & 12 & 7 & 7 & 15 & 15 & 9.3 & \begin{tabular}{|l|}
9.3 \\
\end{tabular} & 11 & 12 & 12 & 12 & 16 & 25 & 14 & 14 & 14 & 16 & 6 & 8 & 13 & 18 & $\mathrm{X}$ & $\mathrm{X}$ & 11 & 12 & 22 & 27 \\
\hline Nwp138 & 13 & 14 & 30 & 30 & 10 & 10 & 11 & 12 & 15 & 16 & 7 & \begin{tabular}{|l|}
7 \\
\end{tabular} & 11 & 13 & 9 & 11 & 19 & 24 & 13 & 14.2 & 15 & 16 & 10 & 11 & 11 & 16 & $\mathrm{X}$ & $\mathrm{Y}$ & 8 & 12 & 23 & 25 \\
\hline Nwp139 & 13 & 15 & 30 & 33.2 & 10 & 11 & 7 & 12 & 15 & 16 & 6 & 9.3 & 13 & 13 & 10 & 11 & 20 & 21 & 13 & 16.2 & 18 & 19 & 8 & 99 & 16 & 18 & $\mathrm{X}$ & $\mathrm{Y}$ & 8 & 12 & 23 & 24 \\
\hline Nwp140 & 14 & 14 & 27 & 27 & 9 & 12 & 10 & 10 & 16 & 17 & 8 & 10 & 11 & 11 & 11 & 13 & 16 & 24 & 12 & 12.2 & 16 & 16 & 6 & 8 & 18 & 19 & $\mathrm{X}$ & $\mathrm{X}$ & 12 & 13 & 25 & 44.2 \\
\hline Nwp141 & 12 & 13 & 28 & 28 & 11 & 12 & 7 & 7 & 15 & 15 & 7 & 7 & 11 & 11 & 9 & 11 & 18 & 24 & 13 & 15 & 15 & 17 & 8 & 10 & 17 & 18 & $\mathrm{X}$ & $\mathrm{X}$ & 13 & 13 & 19.2 & 22 \\
\hline Nwp142 & 15 & 15 & 28 & 31 & 7 & 11 & 10 & 12 & 16 & 17 & 6 & 9.3 & 12 & 12 & 10 & 11 & 19 & 21 & 13 & 14 & 19 & 20 & 10 & 11 & 13 & 14 & $\mathrm{X}$ & $\mathrm{X}$ & 11 & 12 & 22 & 24 \\
\hline Nwp143 & 12 & 14 & 30 & 30 & 10 & 11 & 10 & 11 & 14 & 16 & 8 & 9 & 12 & 13 & 9 & 9 & 19 & 22 & 12 & 16 & 16 & 18 & 8 & 10 & 14 & 18 & $\mathrm{X}$ & $\mathrm{X}$ & 12 & 12 & 19 & 22 \\
\hline Nwp144 & 12 & 12 & 28 & 32.2 & 8 & 11 & 10 & 10 & 15 & 16 & 8 & 8 & 11 & 12 & 11 & 13 & 22 & 25 & 12 & 14 & 17 & 17 & 8 & 10 & 12 & 17 & $\mathrm{X}$ & $\mathrm{Y}$ & 8 & 12 & 18.2 & 19 \\
\hline Nwp145 & 15 & 16 & 27.1 & 28 & 9 & 11 & 10 & 12 & 16 & 16 & 7 & 7 & 11 & 12 & 10 & 12 & 21 & 24 & 12 & 14.2 & 16 & 16 & 6 & 9 & 13 & 15 & $\mathrm{X}$ & $\mathrm{X}$ & 11 & 11 & 23 & 25 \\
\hline Nwp146 & 12 & 14 & 28 & 28 & 10 & 10 & 10 & 12 & 16 & 16 & 7 & 8 & 8 & 12 & 11 & 12 & 16 & 21 & 14.2 & 15 & 15 & 18 & 8 & 10 & 15 & 20 & $\mathrm{X}$ & $\mathrm{Y}$ & 10 & 11 & 24 & 27 \\
\hline Nwp147 & 11 & 13 & 31 & 32 & 8 & 8 & 11 & 11 & 15 & 17 & 7 & 8 & 11 & 11 & 13 & 14 & 24 & 25 & 13 & 13.2 & 14 & 16 & 9 & 9 & 17 & 18 & $\mathrm{X}$ & $\mathrm{X}$ & 11 & 12 & 20 & 23 \\
\hline Nwp148 & 14 & 15 & 29 & 32.2 & 9 & 10 & 10 & 12 & 14 & 15 & 6 & 8 & 12 & 13 & 9 & 12 & 16 & 25 & 12 & 14 & 14 & 15 & 9 & 9 & 13 & 15 & $\mathrm{X}$ & $\mathrm{Y}$ & 12 & 13 & 18.2 & 21 \\
\hline Nwp149 & 12 & 14 & 30 & 32.2 & 10 & 10 & 10 & 11 & 14 & 15 & 6 & 7 & 11 & 12 & 9 & 12 & 19 & 25 & 11 & 15 & 15 & 18 & 9 & 10 & 16 & 17 & $\mathrm{X}$ & $\mathrm{X}$ & 12 & 12 & 21 & 22 \\
\hline Nwp150 & 12 & 13 & 28 & 29 & 9 & 9 & 10 & 13 & 15 & 18 & 6 & 9.3 & 11 & 13 & 11 & 12 & 23 & 25 & 14 & 14 & 14 & 17 & 8 & 10 & 12 & 12 & $\mathrm{X}$ & $\mathrm{X}$ & 12 & 14 & 22 & 24 \\
\hline Nwp151 & 14 & 15 & 29 & 30 & 8 & 12 & 13 & 13 & 15 & 16 & 7 & 7 & 11 & 14 & 11 & 12 & 19 & 24 & 13.2 & 15.2 & 17 & 17 & 6 & 11 & 16 & 18 & $X$ & $\mathrm{X}$ & 8 & 13 & 20 & 23 \\
\hline Nwp152 & 12 & 15 & 28 & 30.2 & 9 & 12 & 10 & 11 & 15 & 16 & 7 & 8 & 13 & 13 & 11 & 12 & 19 & 21 & 14 & 15 & 16 & 17 & 8 & 12 & 17 & 19 & $\mathrm{X}$ & $\mathrm{Y}$ & 13 & 14 & 19 & 24 \\
\hline Nwp153 & 14 & 16 & 28 & 29 & 8 & 8 & 11 & 11 & 17 & 17 & 7 & 8 & 11 & 11 & 9 & 10 & 19 & 19 & 12 & 14 & 15 & 15 & 8 & & 15 & 21 & $X$ & $\mathrm{Y}$ & 11 & 13 & 24 & 26 \\
\hline Nwp154 & 12 & 13 & 29 & 30 & 9 & 12 & 10 & 10 & 14 & 18 & 6 & 7 & 11 & 11 & 11 & 13 & 21 & 25 & 14 & 14 & 14 & 17 & 8 & 8 & 13 & 15 & $\mathrm{X}$ & $X$ & 12 & 13 & 22 & 27 \\
\hline Nwp155 & 14 & 15 & 30 & 31.2 & 9 & 11 & 10 & 10 & 16 & 17 & 7 & 9.3 & 11 & 12 & 11 & 12 & 16 & 21 & 13 & 14 & 16 & 16 & 7 & 9 & 15 & 16 & $X$ & $\mathrm{Y}$ & 11 & 11 & 17 & 24 \\
\hline Nwp156 & 15 & 15 & 30 & 30 & 10 & 12 & 10 & 12 & 14 & 14 & 6 & 7 & 11 & 13 & 12 & 12 & 19 & 21 & 11 & 11 & 14 & 15 & 9 & 11 & 13 & 16 & $\mathrm{X}$ & $\mathrm{Y}$ & 11 & 13 & 21 & 22 \\
\hline Nwp157 & 12 & 13 & 30 & 31 & 8 & 9 & 11 & 12 & 16 & 16 & 6 & 7 & 11 & 12 & 10 & 13 & 17 & 18 & 13 & 14.2 & 15 & 18 & 9 & 11 & 16 & 17 & $\mathrm{X}$ & $\mathrm{Y}$ & 13 & 13 & 17.2 & 20 \\
\hline Nwp158 & 13 & 13 & 27 & 28 & 9 & 10 & 10 & 12 & 16 & 17 & 6 & 7 & 11 & 14 & 11 & 13 & 21 & 25 & 13 & 13 & 16 & 17 & 8 & 8 & 14 & 15 & $\mathrm{X}$ & $\mathrm{Y}$ & 8 & 11 & 22 & 26 \\
\hline Nwp159 & 14 & 15 & 28 & 32.2 & 8 & 11 & 7 & 8 & 16 & 17 & 6 & 7 & 12 & 12 & 12 & 13 & 17 & 23 & 13 & 15 & 14 & 17 & 8 & 11 & 15 & 19 & $\mathrm{X}$ & $\mathrm{X}$ & 11 & 13 & 22 & 26 \\
\hline Nwp160 & 14 & 15 & 28 & 28 & 8 & 8 & 12 & 12 & 15 & 15 & 6 & 7 & 11 & 12 & 9 & 11 & 17 & 21 & 11 & 12 & 17 & 18 & 9 & 12 & 12 & 17 & $\mathrm{X}$ & $\mathrm{Y}$ & 12 & 13 & 25 & 26 \\
\hline
\end{tabular}

Supplementary Table 1: Genotype Assignment (Cont'd) 


\begin{tabular}{|c|c|c|c|c|c|c|c|c|c|c|c|c|c|c|c|c|c|c|c|c|c|c|c|c|c|c|c|c|c|c|c|c|}
\hline \multirow[t]{2}{*}{ Sample \# } & \multicolumn{2}{|c|}{ D8S1179 } & \multicolumn{2}{|c|}{ D21S11 } & \multicolumn{2}{|c|}{ D7S820 } & \multicolumn{2}{|c|}{ CSF1PO } & \multicolumn{2}{|c|}{ D3S1358 } & \multicolumn{2}{|c|}{ TH01 } & \multicolumn{2}{|c|}{ D13S317 } & \multicolumn{2}{|c|}{ D16S539 } & \multicolumn{2}{|c|}{ D2S1338 } & \multicolumn{2}{|c|}{ D19S433 } & \multicolumn{2}{|c|}{ vWA } & TP & $\mathbf{O X}$ & D18 & 3551 & AM & IEL & D5 & 8818 & FG & \\
\hline & 1 & 2 & 1 & 2 & 1 & 2 & 1 & 2 & 1 & 2 & 1 & 2 & 1 & 2 & 1 & 2 & 1 & 2 & 1 & 2 & 1 & 2 & 1 & 2 & 1 & 2 & 1 & 2 & 1 & \begin{tabular}{|l}
2 \\
\end{tabular} & 1 & 2 \\
\hline Nwp161 & 13 & 14 & 30 & 32.2 & 10 & 12 & 11 & 12 & 16 & 16 & 7 & 8 & 11 & 13 & 11 & 11 & 19 & 24 & 13 & 13 & 16 & 17 & 9 & 11 & 13 & 18 & $\mathrm{X}$ & $\mathrm{X}$ & 12 & 13 & 19 & 21 \\
\hline Nwp162 & 13 & 15 & 29 & 30 & 8 & 10 & 11 & 13 & 17 & 18 & 9 & 9.3 & 11 & 11 & 13 & 14 & 17 & 23 & 13 & 14.2 & 16 & 19 & 8 & 8 & 14 & 16 & $\mathrm{X}$ & $\mathrm{X}$ & 13 & 13 & 22 & 25 \\
\hline Nwp165 & 14 & 15 & 29 & 30 & 10 & 12 & 9 & 12 & 16 & 17 & 6 & 9 & 11 & 15 & 9 & 11 & 16 & 22 & 11 & 17.2 & 17 & 19 & 6 & 6 & 18 & 19 & $\mathrm{X}$ & $\mathrm{X}$ & 12 & 13 & 18.2 & 25 \\
\hline Nwp166 & 13 & 15 & 29 & 30 & 10 & 12 & 8 & 12 & 15 & 17 & 7 & 8 & 12 & 12 & 10 & 11 & 21 & 23 & 14 & 15.2 & 14 & 15 & 11 & 11 & 12 & 22 & $\mathrm{X}$ & $\mathrm{X}$ & 9 & 12 & 25 & 25 \\
\hline Nwp167 & 14 & 14 & 29 & 30 & 10 & 11 & 11 & 12 & 16 & 16 & 6 & 8 & 11 & 13 & 12 & 14 & 20 & 21 & 14 & 16.2 & 14 & 16 & 7 & 9 & 17 & 17 & $\mathrm{X}$ & $\mathrm{X}$ & 11 & 13 & 22 & 22 \\
\hline Nwp168 & 14 & 15 & 29 & 30.2 & 9 & 12 & 11 & 11 & 15 & 17 & 7 & 9 & 12 & 13 & 11 & 12 & 19 & 19 & 13 & 14.2 & 16 & 18 & 9 & 11 & 18 & 18 & $\mathrm{X}$ & $\mathrm{X}$ & 12 & 12 & 20 & 25 \\
\hline Nwp170 & 16 & 16 & 29 & 31 & 10 & 10 & 12 & 14 & 15 & 17 & 7 & 8 & 10 & 12 & 9 & 12 & 17 & 20 & 11 & 15 & 16 & 18 & 8 & 11 & 15 & 17 & $\mathrm{X}$ & $X$ & 13 & 13 & 21 & 22 \\
\hline Nwp171 & 14 & 15 & 29 & 30.2 & 8 & 11 & 10 & 10 & 15 & 16 & 7 & 9.3 & 11 & 12 & 10 & 12 & 19 & 19 & 12 & 14.2 & 15 & 19 & 8 & 8 & 13 & 20 & $\mathrm{X}$ & $\mathrm{X}$ & 12 & 13 & 21 & 22 \\
\hline Nwp172 & 14 & 14 & 28 & 31.2 & 8 & 11 & 10 & 11 & 17 & 18 & 6 & 9.3 & 10 & 11 & & 11 & 19 & 21 & 12 & 13 & 16 & 18 & 8 & 10 & 14 & 15 & $\mathrm{X}$ & $\mathrm{Y}$ & 8 & 11 & 23 & 23 \\
\hline Nwp173 & 13 & 14 & 28 & 33.2 & 7 & 10 & 10 & 11 & 15 & 15 & 7 & 8 & 12 & 13 & 9 & 11 & 17 & 23 & 13 & 14 & 16 & 17 & 8 & 9 & 15 & 20 & $\mathrm{X}$ & $\mathrm{Y}$ & 12 & 12 & 20 & 23 \\
\hline Nwp174 & 15 & 18 & 31 & 33.2 & 8 & 9 & 8 & 11 & 14 & 15 & 7 & 7 & 11 & 12 & 9 & 12 & 21 & 22 & 12 & 12.2 & 15 & 16 & 6 & 9 & 12 & 20 & $\mathrm{X}$ & $\mathrm{Y}$ & 11 & 11 & 18 & 19 \\
\hline Nwp175 & 13 & 16 & 27 & 29 & 10 & 10 & 8 & 11 & 16 & 16 & 9 & 9.3 & 8 & 9 & 12 & 13 & 16 & 19 & 12.2 & 15.2 & 17 & 18 & 9 & 9 & 17 & 18 & $\mathrm{X}$ & $\mathrm{Y}$ & 12 & 13 & 22 & 25 \\
\hline Nwp178 & 12 & 14 & 29 & 30 & 10 & 14 & 8 & 12 & 15 & 15 & 7 & 8 & 12 & 13 & 11 & 12 & 19 & 23 & 13 & 14 & 16 & 18 & 8 & 11 & 15 & 16 & $\mathrm{X}$ & $\mathrm{Y}$ & 11 & 11 & 20 & 25 \\
\hline Nwp180 & 12 & 14 & 28 & 30 & 8 & 9 & 12 & 13 & 14 & 15 & 8 & 9 & 11 & 13 & 8 & 9 & 17 & 18 & 12 & 15.2 & 15 & 16 & 10 & 11 & 16 & 17 & $\mathrm{X}$ & $\mathrm{X}$ & 10 & 12 & 19 & 22 \\
\hline Nwp181 & 12 & 15 & 30 & 32 & 8 & 10 & 11 & 12 & 15 & 17 & 7 & 9 & 11 & 12 & 11 & 11 & 17 & 26 & 13 & 13 & 16 & 16 & 10 & 11 & 16 & 20 & $\mathrm{X}$ & $\mathrm{Y}$ & 11 & 11 & 20 & 21 \\
\hline Nwp182 & 13 & 14 & 30 & 31 & 8 & 10 & 7 & 12 & 16 & 17 & 7 & 9 & 11 & 12 & 8 & 8 & 19 & 19 & 14 & 14.2 & 17 & 17 & 10 & 10 & 19 & 22 & $\mathrm{X}$ & $\mathrm{X}$ & 12 & 12 & 20 & 27 \\
\hline Nwp183 & 11 & 11 & 28 & 29 & 9 & 9 & 12 & 12 & 16 & 18 & 7 & 7 & 12 & 13 & 12 & 12 & 21 & 25 & 10 & 13 & 17 & 19 & 9 & 9 & 15 & 17 & $\mathrm{X}$ & $\mathrm{X}$ & 8 & 13 & 23 & 29 \\
\hline Nwp184 & 14 & 16 & 28 & 34 & 9 & 11 & 10 & 12 & 15 & 17 & 7 & 8 & 11 & 12 & 12 & 13 & 17 & 19 & 14 & 16.2 & 15 & 16 & 8 & 11 & 17 & 19 & $\mathrm{X}$ & $\mathrm{X}$ & 10 & 12 & 21 & 22 \\
\hline Nwp185 & 11 & 14 & 28 & 30.2 & 9 & 10 & 11 & 11 & 14 & 18 & 7 & 8 & 11 & 12 & 9 & 10 & 22 & 23 & 11.2 & 15 & 16 & 19 & 10 & 10 & 12 & 15 & $\mathrm{X}$ & $X$ & 13 & 13 & 21 & 22 \\
\hline Nwp186 & 12 & 15 & 28 & 29 & 10 & 10 & 11 & 12 & 15 & 16 & 8 & 9 & 11 & 13 & 10 & 13 & 19 & 23 & 13 & 13 & 15 & 18 & 11 & 11 & 15 & 16 & $\mathrm{X}$ & $\mathrm{Y}$ & 13 & 13 & 18.2 & 22 \\
\hline Nwp187 & 12 & 14 & 28 & 29 & 8 & 9 & 12 & 12 & 16 & 17 & 7 & 9 & 12 & 13 & 9 & 12 & 24 & 25 & 13 & 14 & 16 & 17 & 8 & 12 & 14 & 16 & $\mathrm{X}$ & $\mathrm{X}$ & 13 & 13 & 22 & 24 \\
\hline Nwp188 & 14 & 15 & 32.2 & 32.2 & 9 & 10 & 11 & 13 & 16 & 17 & 9 & 9.3 & 11 & 11 & 10 & 12 & 16 & 19 & 13 & 14.2 & 15 & 19 & 8 & 11 & 16 & 19 & $\mathrm{X}$ & $\mathrm{Y}$ & 12 & 13 & 21 & 24 \\
\hline Nwp189 & 12 & 13 & 30 & 33.2 & 10 & 11 & 7 & 12 & 14 & 16 & 6 & 9 & 11 & 12 & 12 & 12 & 16 & 19 & 12 & 13 & 16 & 18 & 6 & 9 & 16 & 17 & $\mathrm{X}$ & $\mathrm{X}$ & 12 & 12 & 22 & 26 \\
\hline Nwp190 & 15 & 15 & 28 & 29 & 10 & 12 & 11 & 12 & 17 & 17 & 7 & 9.3 & 10 & 11 & 11 & 11 & 21 & 24 & 11 & 13.2 & 15 & 20 & 6 & 11 & 16 & 17 & $\mathrm{X}$ & $\mathrm{Y}$ & 11 & 12 & 23 & 26 \\
\hline Nwp191 & 13 & 14 & 29 & 30 & 8 & 10 & 11 & 11 & 15 & 15 & 7 & 8 & 11 & 11 & 11 & 12 & 19 & 24 & 12 & 14.2 & 16 & 18 & 7 & 10 & 18 & 19 & $\mathrm{X}$ & $\mathrm{X}$ & 10 & 11 & 22 & 24 \\
\hline Nwp193 & 14 & 14 & 30 & 30 & 10 & 10 & 12 & 12 & 15 & 15 & 7 & 9.3 & 11 & 13 & 11 & 11 & 17 & 17 & 12.2 & 13 & 15 & 17 & 8 & 11 & 15 & 18 & $\mathrm{X}$ & $\mathrm{Y}$ & 11 & 13 & 22 & 23 \\
\hline Nwp194 & 13 & 14 & 28 & 29.2 & 10 & 10 & 7 & 8 & 15 & 15 & 7 & 8 & 10 & 12 & 9 & 12 & 19 & 20 & 13 & 14 & 17 & 19 & 8 & 8 & 13 & 17 & $\mathrm{X}$ & $X$ & 11 & 13 & 24 & 25 \\
\hline Nwp195 & 12 & 14 & 28 & 30 & 10 & 10 & 12 & 12 & 15 & 17 & 9.3 & 9.3 & 12 & 12 & 9 & 12 & 19 & 19 & 12 & 13 & 14 & 17 & 9 & 10 & 13 & 16 & $\mathrm{X}$ & $\mathrm{Y}$ & 8 & 12 & 24 & 26 \\
\hline Nwp196 & 15 & 16 & 31.2 & 32.2 & 8 & 11 & 11 & 12 & 15 & 16 & 6 & 9 & 10 & 11 & 9 & 13 & 17 & 23 & 13 & 14 & 16 & 18 & 6 & 8 & 13 & 15 & $\mathrm{X}$ & $\mathrm{X}$ & 11 & 12 & 21 & 26 \\
\hline Nwp197 & 14 & 15 & 28 & 28 & 10 & 10 & 10 & 13 & 15 & 15 & 6 & 8 & 12 & 13 & 11 & 11 & 20 & 24 & 15 & 15 & 16 & 17 & 8 & 9 & 15 & 15 & $\mathrm{X}$ & $\mathrm{Y}$ & 12 & 13 & 20 & 24 \\
\hline Nwp198 & 10 & 15 & 32.2 & 32.2 & 9 & 10 & 8 & 10 & 15 & 16 & 9 & 9.3 & 9 & 12 & 11 & 11 & 24 & 26 & 13 & 13 & 16 & 17 & 9 & 9 & 15 & 21 & $\mathrm{X}$ & $\mathrm{X}$ & 8 & 12 & 23 & 24 \\
\hline Nwp199 & 13 & 15 & 28 & 33.2 & 8 & 9 & 10 & 11 & 17 & 17 & 8 & 9 & 12 & 13 & 9 & 11 & 21 & 23 & 14 & 15.2 & 15 & 17 & 6 & 11 & 14 & 21 & $\mathrm{X}$ & $\mathrm{Y}$ & 11 & 13 & 23 & 24 \\
\hline Nwp200 & 14 & 14 & 28 & 28 & 8 & 9 & 10 & 12 & 16 & 17 & 7 & 8 & 9 & 14 & 13 & 13 & 17 & 22 & 11.2 & 16 & 17 & 17 & 8 & 8 & 14 & 17 & $\mathrm{X}$ & $\mathrm{Y}$ & 11 & 12 & 18 & 24 \\
\hline Nwp201 & 13 & 14 & 28 & 28 & 8 & 8 & 12 & 12 & 14 & 16 & 7 & 8 & 12 & 14 & 9 & 12 & 17 & 21 & 13 & 14.2 & 14 & 18 & 11 & 11 & 18 & 19 & $\mathrm{X}$ & $\mathrm{Y}$ & 11 & 11 & 22 & 25 \\
\hline Nwp202 & 14 & 14 & 27 & 28 & 10 & 10 & 11 & 12 & 15 & 15 & 8 & 9.3 & 12 & 14 & 12 & 13 & 17 & 21 & 13 & 16.2 & 15 & 19 & 8 & 11 & 13 & 17 & $\mathrm{X}$ & $\mathrm{Y}$ & 11 & 12 & 20 & 24 \\
\hline Nwp203 & 14 & 15 & 30 & 32.2 & 10 & 10 & 13 & 13 & 14 & 15 & 6 & 9.3 & 11 & 14 & 11 & 13 & 17 & 19 & 11 & 13 & 16 & 17 & 8 & 11 & 18 & 19 & $\mathrm{X}$ & $\mathrm{Y}$ & 10 & 10 & 23 & 24 \\
\hline Nwp204 & 13 & 13 & 28 & 30 & 10 & 10 & 11 & 11 & 16 & 17 & 7 & 7 & 12 & 12 & 9 & 13 & 19 & 22 & 14 & 15.2 & 14 & 19 & 8 & 11 & 15 & 19 & $\mathrm{X}$ & $X$ & 12 & 13 & 21 & 23 \\
\hline Nwp206 & 13 & 15 & 29 & 31 & 10 & 12 & 8 & 11 & 15 & 16 & 7 & 7 & 12 & 12 & 11 & 12 & 19 & 21 & 11 & 14.2 & 17 & 19 & 6 & 11 & 15 & 20 & $\mathrm{X}$ & $\mathrm{Y}$ & 12 & 13 & 21 & 23 \\
\hline Nwp208 & 14 & 15 & 27 & 30 & 10 & 10 & 11 & 12 & 16 & 17 & 7 & 8 & 12 & 12 & 11 & 13 & 25 & 25 & 12 & 12.2 & 16 & 17 & 9 & 11 & 16 & 20 & $\mathrm{X}$ & $\mathrm{Y}$ & 11 & 13 & 22 & 24 \\
\hline
\end{tabular}

Supplementary Table 1: Genotype Assignment (Cont'd) 


\begin{tabular}{|c|c|c|c|c|c|c|c|c|c|c|c|c|c|c|c|c|c|c|c|c|c|c|c|c|c|c|c|c|c|c|c|c|}
\hline Sample \# & S & & D21 & S11 & D7S & 820 & CSF & PO & D3S & 358 & TH & & D13S & 3317 & D & 539 & D2S & 1338 & D19: & 433 & vW & VA & TP( & OX & D18 & 3551 & $\mathbf{A M}$ & & D5S & 818 & FC & $\mathrm{SA}$ \\
\hline & 1 & 2 & 1 & 2 & 1 & 2 & 1 & 2 & 1 & 2 & 1 & 2 & 1 & 2 & 1 & 2 & 1 & 2 & 1 & 2 & 1 & 2 & 1 & 3 & 1 & 2 & 1 & 2 & 1 & 2 & 1 & 2 \\
\hline Nwp209 & 13 & 15 & 29 & 29 & 12 & 12 & & 8 & 14 & 16 & 7 & 9 & 9 & 12 & 10 & 11 & 22 & 23 & 13 & 13.2 & 16 & 18 & 11 & 11 & 15 & 16 & X & Y & 12 & 13 & 18 & 27 \\
\hline Nwp210 & 11 & 15 & 30 & 31 & 10 & 10 & 10 & 10 & 17 & 18 & 7 & 7 & 11 & 11 & 9 & 11 & 19 & 19 & 13 & 13 & 15 & 15 & 6 & 8 & 16 & 18 & $X$ & Y & 11 & 11 & 21 & 25 \\
\hline Nwp211 & 14 & 15 & 29 & 30.3 & 8 & 10 & 10 & 11 & 13 & 17 & 8 & 8 & 11 & 14 & 10 & 13 & 17 & 21 & 11 & 15 & 17 & 18 & 11 & 11 & 16 & 18 & $\mathrm{X}$ & $\mathrm{Y}$ & 0 & 12 & 22 & 23 \\
\hline 0212 & 13 & 14 & 36 & 31 & 11 & 12 & 11 & 1. & 15 & 16 & 9 & 9 & 1 & & & 1 & & & & 15.2 & 15 & 1 & & & 16 & 17 & $\mathrm{x}$ & $\mathrm{Y}$ & 1 & 13 & 1 & 23 \\
\hline & 12 & 15 & 25 & 3 & 10 & 11 & 11 & 11 & & 14 & 9 & 9.3 & 11 & & 11 & 1 & & & & & 15 & & & & 5 & 8 & & $\mathrm{Y}$ & & 2 & 0 & 24 \\
\hline & 11 & 14 & 28 & 2 & 10 & 11 & & 11 & 1 & 1 & 7 & 8 & 11 & 1 & & 10 & 22 & 2 & & & 15 & 15 & & 11 & 17 & 18 & & $\mathrm{Y}$ & 2 & 3 & 23 & 24 \\
\hline & 1 & 13 & 31 & 32.2 & 12 & 12 & 1 & 11 & & 1 & 8 & & 11 & 1 & & 13 & 17 & 1 & & & 15 & 15 & & 11 & 18 & 19 & & $\mathrm{Y}$ & 2 & 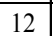 & 19 & 25 \\
\hline $\mathrm{Nw}$ & 14 & 14 & 30 & 30.2 & 10 & 12 & 1 & 12 & 14 & 1. & 6 & 7 & 13 & 12 & & 13 & 22 & 2 & 14 & 2 & 15 & 17 & & 11 & 15 & 17 & & $X$ & 12 & 12 & 19 & 22 \\
\hline Nwp219 & 11 & 12 & 27 & 28 & 9 & 10 & 10 & 11 & 14 & 18 & 6 & 8 & 12 & 12 & & 11 & 20 & 2 & 12 & 12.2 & 15 & 16 & 8 & 11 & 11 & 16 & $\mathrm{X}$ & $\mathrm{Y}$ & 12 & 13 & 21 & 25 \\
\hline Nwp220 & 11 & 15 & 31.2 & 35 & 8 & 9 & 10 & 11 & 15 & 17 & 7 & 8 & 12 & 13 & 11 & 12 & 20 & 23 & 13 & 14 & 15 & 18 & 8 & & 15 & 16 & $\mathrm{X}$ & $\mathrm{Y}$ & 9 & 13 & 22 & 23.3 \\
\hline 221 & 12 & 13 & 28 & 29 & 9 & 11 & 8 & 12 & 16 & 16 & 7 & 7 & 10 & 11 & 9 & 11 & 23 & 2. & 13 & 15.2 & 16 & 17 & 9 & 11 & 16 & 17 & $\mathrm{X}$ & Y & 8 & 14 & 21 & 24 \\
\hline 223 & 13 & 14 & 28 & 30 & 8 & 9 & 8 & 12 & 15 & 17 & 7 & 9 & 11 & 12 & 11 & 13 & 21 & 2 & 13 & 13 & 16 & 17 & 8 & 11 & 15 & 17 & $\mathrm{X}$ & Y & 8 & 11 & 22 & 24 \\
\hline Nwp224 & 14 & 16 & 28 & 32.2 & 10 & 10 & 8 & 12 & 14 & 1 & 7 & 9 & 12 & 1 & 9 & 12 & 21 & 2 & 13 & 13.2 & 17 & 19 & 6 & & 15 & 17 & $X$ & $\mathrm{Y}$ & 12 & 13 & 20 & 22 \\
\hline Nwp225 & 1 & 15 & 28 & 28 & 10 & 11 & 10 & 12 & & 1 & 7 & 7 & 11 & & 12 & & & & & & 15 & & & & 14 & 6 & $X$ & $\mathrm{Y}$ & 1 & 2 & 19 & 22 \\
\hline & 1 & 14 & 2 & & 8 & 10 & & & & & 7 & 8 & & & & & & & & & 1. & & 9 & & & & & & & 3 & 25 & 0.2 \\
\hline & 1 & 1 & 25 & 31. & 8 & 11 & 1 & 10 & 1 & & 6 & 6 & 11 & & & & & & & & 16 & & 8 & & 18 & 19 & & $\mathrm{X}$ & & 11 & 18.2 & 22 \\
\hline Nwp612 & 13 & 15 & 29 & 30 & 10 & 10 & 11 & 11 & 18 & 18 & 9.3 & 9.3 & 12 & 12 & 12 & 12 & 23 & 25 & 14 & 16.2 & 16 & 18 & 8 & 8 & 16 & 17 & $X$ & $X$ & 12 & 13 & 22 & 25 \\
\hline Nwp613 & 12 & 15 & 29 & 32.2 & 11 & 12 & 10 & 10 & 18 & 18 & 7 & 9.3 & 8 & 11 & 11 & 13 & 18 & 20 & 13 & 14 & 17 & 17 & 8 & 11 & 12 & 18 & $\mathrm{X}$ & $X$ & 13 & 13 & 20 & 21 \\
\hline Nwp614 & 13 & 14 & 29 & 30 & 8 & 9 & 11 & 12 & 16 & 18 & 7 & 9 & 8 & 8 & 10 & 13 & 19 & 23 & 13 & 14 & 16 & 17 & 8 & 8 & 13 & 15 & $\mathrm{X}$ & $\mathrm{X}$ & 12 & 13 & 22 & 23 \\
\hline Nwp615 & 15 & 16 & 31.2 & 31.2 & 10 & 11 & 11 & 12 & 17 & 18 & 7 & 9.3 & 11 & 11 & 9 & 12 & 17 & 20 & 13 & 14 & 18 & 18 & 8 & 8 & 15 & 17 & $\mathrm{X}$ & $\mathrm{Y}$ & 12 & 12 & 23 & 24 \\
\hline Nwp616 & 14 & 14 & 28 & 29 & 9 & 11 & 10 & 10 & 15 & 17 & 7 & 8 & 11 & 12 & 11 & 11 & 16 & 20 & 14.2 & 16.2 & 16 & 16 & 8 & 11 & 15 & 15 & $\mathrm{X}$ & $X$ & 10 & 13 & 23 & 24 \\
\hline Nwp617 & 14 & 15 & 29 & 31 & 11 & 11 & 8 & 12 & 15 & 17 & 7 & 9.3 & 12 & 12 & 12 & 12 & 20 & 24 & 14.2 & 15 & 14 & 18 & 9 & 12 & 14 & 17 & $\mathrm{X}$ & $\mathrm{X}$ & 11 & 12 & 22 & 25 \\
\hline Nwp618 & 11 & 14 & 30 & 31.2 & 10 & 10 & 11 & 11 & 15 & 15 & 7 & 9.3 & 12 & 13 & 10 & 11 & 20 & 24 & 12 & 12.2 & 16 & 16 & 11 & 11 & 16 & 16 & $\mathrm{X}$ & $X$ & 8 & 12 & 20 & 22 \\
\hline
\end{tabular}

Supplementary Table 1: Genotype Assignment (Cont'd) 


\begin{tabular}{|c|c|c|c|c|c|c|c|c|c|c|c|c|c|c|}
\hline allele & 6 & 6.3 & 7 & 8 & 9 & 9.3 & 10 & 11 & 11.2 & 12 & 12.2 & 13 & 13.2 & 14 \\
\hline D8S1179 & & & & 0.0023 & & & 0.0136 & 0.0860 & & 0.1154 & & 0.1900 & & 0.3032 \\
\hline \multicolumn{15}{|l|}{ D21S11 } \\
\hline D7S820 & & & 0.0068 & 0.2104 & 0.1538 & & 0.3371 & 0.1584 & & 0.1176 & & 0.0136 & & 0.0023 \\
\hline CSF1PO & 0.0023 & & 0.0747 & 0.0656 & 0.0204 & & 0.2376 & 0.2557 & & 0.2896 & & 0.0475 & & 0.0045 \\
\hline D3S1358 & & & & & & & & & & 0.0068 & & 0.0023 & & 0.0882 \\
\hline TH01 & 0.1425 & 0.0023 & 0.3869 & 0.2240 & 0.1199 & 0.1199 & 0.0045 & & & & & & & \\
\hline D13S317 & & & 0.0023 & 0.0317 & 0.0181 & & 0.0339 & 0.3122 & & 0.3869 & & 0.1538 & & 0.0566 \\
\hline D16S539 & 0.0023 & & & 0.0204 & 0.1561 & & 0.1176 & 0.2919 & & 0.2240 & & 0.1561 & & 0.0294 \\
\hline \multicolumn{15}{|l|}{ D2S1338 } \\
\hline D19S433 & & & & & 0.0023 & & 0.0113 & 0.0566 & 0.0068 & 0.1244 & 0.0385 & 0.3122 & 0.0362 & 0.2081 \\
\hline vWA & & & & & & & & 0.0023 & & 0.0045 & & 0.0113 & & 0.0633 \\
\hline TPOX & 0.0747 & & 0.0204 & 0.3122 & 0.2059 & & 0.1131 & 0.2421 & & 0.0317 & & & & \\
\hline D18S51 & & & & & & & & 0.0045 & & 0.0566 & & 0.0814 & 0.0090 & 0.0475 \\
\hline D5S818 & & & 0.0045 & 0.0701 & 0.0068 & & 0.0520 & 0.2557 & & 0.3235 & & 0.2692 & & 0.0136 \\
\hline \multicolumn{15}{|l|}{ FGA } \\
\hline allele & 14.1 & 14.2 & 15 & 15.2 & 16 & 16.1 & 16.2 & 17 & 17.2 & 18 & 18.2 & 19 & 19.2 & 20 \\
\hline D8S1179 & & & 0.2195 & & 0.0543 & & & 0.0090 & & 0.0068 & & & & \\
\hline \multicolumn{15}{|l|}{ D21S11 } \\
\hline \multicolumn{15}{|l|}{ D7S820 } \\
\hline CSF1PO & & & 0.0023 & & & & & & & & & & & \\
\hline D3S1358 & & & 0.3077 & 0.0045 & 0.3100 & & & 0.1968 & & 0.0814 & & 0.0023 & & \\
\hline \multicolumn{15}{|l|}{ TH01 } \\
\hline D13S317 & & & 0.0045 & & & & & & & & & & & \\
\hline D16S539 & & & 0.0023 & & & & & & & & & & & \\
\hline D2S1338 & & & & & 0.0588 & & & 0.1425 & & 0.0452 & & 0.1833 & & 0.0837 \\
\hline D19S433 & 0.0023 & 0.0566 & 0.0747 & 0.0385 & 0.0068 & & 0.0204 & 0.0023 & 0.0023 & & & & & \\
\hline vWA & & & 0.2014 & & 0.2534 & & & 0.2376 & & 0.1403 & & 0.0701 & & 0.0113 \\
\hline \multicolumn{15}{|l|}{ TPOX } \\
\hline D18S51 & & 0.0068 & 0.1742 & 0.0068 & 0.1584 & & & 0.1652 & & 0.1471 & & 0.0747 & & 0.0362 \\
\hline D5S818 & & & 0.0045 & & & & & & & & & & & \\
\hline FGA & & & & & & 0.0023 & & 0.0023 & 0.0045 & 0.0068 & 0.0271 & 0.0543 & 0.0023 & 0.0701 \\
\hline
\end{tabular}

Supplementary Table 2: New Providence Allelic Frequencies $(\mathbf{n}=221)$ 


\begin{tabular}{|c|c|c|c|c|c|c|c|c|c|c|c|c|c|c|}
\hline allele & $\mathbf{2 1}$ & $\mathbf{2 2}$ & $\mathbf{2 2 . 2}$ & $\mathbf{2 3}$ & $\mathbf{2 3 . 3}$ & $\mathbf{2 4}$ & $\mathbf{2 5}$ & $\mathbf{2 6}$ & $\mathbf{2 7}$ & $\mathbf{2 7 . 1}$ & $\mathbf{2 8}$ & $\mathbf{2 9}$ & $\mathbf{2 9 . 2}$ & $\mathbf{3 0}$ \\
\hline D8S1179 & & & & & & & & & & & & & & \\
\hline D21S11 & & & & & & & & & 0.0385 & 0.0023 & 0.2353 & 0.2127 & 0.0023 & 0.1991 \\
\hline D7S820 & & & & & & & & & & & & & & \\
\hline CSF1PO & & & & & & & & & & & & & & \\
\hline D3S1358 & & & & & & & & & & & & & & \\
\hline TH01 & & & & & & & & & & & & & & \\
\hline D13S317 & & & & & & & & & & & & & & \\
\hline D16S539 & & & & & & & & & & & & & & \\
\hline D2S1338 & 0.1380 & 0.0995 & & 0.0882 & & 0.0792 & 0.0611 & 0.0181 & 0.0023 & & & & & \\
\hline D19S433 & & & & & & & & & & & & & & \\
\hline VWA & 0.0045 & & & & & & & & & & & & & \\
\hline TPOX & & & & & & & & & & & & & & \\
\hline D18S51 & 0.0136 & 0.0181 & & & & & & & & & & & & \\
\hline D5S818
\end{tabular}

\begin{tabular}{|c|c|c|c|c|c|c|c|c|c|c|c|c|c|}
\hline allele & $\mathbf{3 0 . 2}$ & $\mathbf{3 0 . 3}$ & $\mathbf{3 1}$ & $\mathbf{3 1 . 2}$ & $\mathbf{3 2}$ & $\mathbf{3 2 . 2}$ & $\mathbf{3 3 . 2}$ & $\mathbf{3 4}$ & $\mathbf{3 4 . 2}$ & $\mathbf{3 5}$ & $\mathbf{3 7}$ & $\mathbf{4 4 . 2}$ & $\mathbf{4 6 . 2}$ \\
\hline D8S1179 & & & & & & & & & & & & & \\
\hline D21S11 & 0.0158 & 0.0023 & 0.0792 & 0.0430 & 0.0158 & 0.0747 & 0.0249 & 0.0158 & & 0.0317 & 0.0068 & & \\
\hline D7S820 & & & & & & & & & & & & & \\
\hline CSF1PO & & & & & & & & & & & & & \\
\hline D3S1358 & & & & & & & & & & & & & \\
\hline TH01 & & & & & & & & & & & & & \\
\hline D13S317 & & & & & & & & & & & & \\
\hline D16S539 & & & & & & & & & & & & \\
\hline D2S1338 & & & & & & & & & & & & & \\
\hline D19S433 & & & & & & & & & & & & & \\
\hline VWA & & & & & & & & & & & & & \\
\hline TPOX & & & & & & & & & & & & \\
\hline D18S51 & & & & & & & & & & & & \\
\hline D5S818 & & & & & & & & & & & & & \\
\hline FGA & 0.0023 & & & 0.0023 & & & & & 0.0045 & & & 0.0023 & 0.0023 \\
\hline
\end{tabular}

Supplementary Table 2: New Providence Allelic Frequencies (n=221) (Cont'd) 


\begin{tabular}{|c|c|c|c|c|c|c|c|c|c|}
\hline & $\mathbf{H}_{\mathbf{0}}$ & $\mathbf{H}_{\mathbf{e}}$ & $\mathbf{H W E}$ & GDI & MP & PD & PIC & PE & TPI \\
\hline D8S1179 & 0.8326 & 0.8017 & 0.2387 & 0.8017 & 0.0731 & 0.9269 & 0.7721 & 0.6608 & 2.9865 \\
\hline D21S11 & 0.7783 & 0.8441 & 0.1121 & 0.8441 & 0.0442 & 0.9558 & 0.8240 & 0.5594 & 2.2551 \\
\hline D7S820 & 0.7376 & 0.8287 & 0.2612 & 0.7810 & 0.0794 & 0.9206 & 0.7468 & 0.4887 & 1.9052 \\
\hline CSF1PO & 0.7195 & 0.8057 & 0.0149 & 0.7835 & 0.0850 & 0.9150 & 0.7487 & 0.4590 & 1.7823 \\
\hline D3S1358 & 0.7557 & 0.7577 & 0.2439 & 0.7577 & 0.1033 & 0.8967 & 0.7163 & 0.5195 & 2.0463 \\
\hline TH01 & 0.7557 & 0.7619 & 0.8187 & 0.7528 & 0.1030 & 0.8970 & 0.7148 & 0.5195 & 2.0463 \\
\hline D13S317 & 0.7104 & 0.7767 & 0.0832 & 0.7251 & 0.1239 & 0.8761 & 0.6795 & 0.4445 & 1.7266 \\
\hline D16S539 & 0.7919 & 0.8076 & 0.5014 & 0.8026 & 0.0719 & 0.9281 & 0.7722 & 0.5840 & 2.4022 \\
\hline D2S1338 & 0.8552 & 0.8925 & 0.0225 & 0.8885 & 0.0277 & 0.9723 & 0.8758 & 0.7051 & 3.4531 \\
\hline D19S433 & 0.7873 & 0.8292 & 0.0421 & 0.8287 & 0.0530 & 0.9470 & 0.8085 & 0.5757 & 2.3511 \\
\hline vWA & 0.7964 & 0.8117 & 0.8150 & 0.8117 & 0.0630 & 0.9370 & 0.7831 & 0.5923 & 2.4556 \\
\hline TPOX & 0.6833 & 0.7835 & 0.0459 & 0.7835 & 0.0776 & 0.9224 & 0.7490 & 0.4028 & 1.5786 \\
\hline D18S51 & 0.8959 & 0.8815 & 0.5869 & 0.8780 & 0.0321 & 0.9679 & 0.8635 & 0.7871 & 4.8043 \\
\hline D5S818 & 0.7330 & 0.7513 & 0.5011 & 0.7513 & 0.1038 & 0.8962 & 0.7074 & 0.4812 & 1.8729 \\
\hline FGA & 0.9140 & 0.8856 & 0.8808 & 0.8831 & 0.0289 & 0.9711 & 0.8698 & 0.8242 & 5.8158 \\
\hline
\end{tabular}

Supplementary Table 3: Forensic parameters for the New Providence population across the 15 Identifiler loci 


\begin{tabular}{|c|c|c|c|}
\hline \multirow[b]{2}{*}{ Locus } & \multicolumn{3}{|c|}{ Afro-American } \\
\hline & $\mathbf{G}_{\mathbf{s t}}$ & $\mathbf{H}_{\mathrm{s}}$ & $\mathbf{H}_{\mathrm{t}}$ \\
\hline D8S1179 & 0.00348 & 0.79481 & 0.79758 \\
\hline D21S11 & 0.00132 & 0.84440 & 0.84551 \\
\hline D7S820 & 0.00229 & 0.78374 & 0.78554 \\
\hline CSF1PO & 0.00066 & 0.77993 & 0.78044 \\
\hline D3S1358 & 0.00353 & 0.75652 & 0.75920 \\
\hline TH01 & 0.00135 & 0.74817 & 0.74918 \\
\hline D13S317 & 0.00096 & 0.71751 & 0.71820 \\
\hline D16S539 & 0.00231 & 0.79199 & 0.79382 \\
\hline D2S1338 & 0.00231 & 0.88917 & 0.89123 \\
\hline D19S433 & 0.00217 & 0.83525 & 0.83706 \\
\hline vWA & 0.00147 & 0.81372 & 0.81492 \\
\hline TPOX & 0.00187 & 0.77164 & 0.77308 \\
\hline D18S51 & 0.00179 & 0.87818 & 0.87975 \\
\hline D5S818 & 0.00216 & 0.75284 & 0.75447 \\
\hline FGA & 0.00330 & 0.87295 & 0.87584 \\
\hline All Loci & 0.00208 & 0.80205 & 0.80372 \\
\hline
\end{tabular}

\begin{tabular}{ccc}
\hline \multicolumn{3}{c}{ African w/o Madagascar } \\
\hline $\mathbf{G}_{\text {st }}$ & $\mathbf{H}_{\mathbf{s}}$ & $\mathbf{H}_{\mathbf{t}}$ \\
\hline 0.00911 & 0.77616 & 0.78329 \\
0.00749 & 0.85154 & 0.85797 \\
0.00882 & 0.75652 & 0.76325 \\
0.01508 & 0.77298 & 0.78481 \\
0.00910 & 0.73288 & 0.73960 \\
0.00889 & 0.73109 & 0.73765 \\
0.00554 & 0.72035 & 0.72436 \\
0.00810 & 0.78449 & 0.79090 \\
0.00903 & 0.88566 & 0.89373 \\
0.00892 & 0.82476 & 0.83219 \\
0.00701 & 0.81647 & 0.82223 \\
0.01072 & 0.77671 & 0.78512 \\
0.00723 & 0.86463 & 0.87093 \\
0.00380 & 0.74904 & 0.75190 \\
0.00619 & 0.87787 & 0.88334 \\
0.00833 & 0.79474 & 0.80142
\end{tabular}

\begin{tabular}{ccc}
\hline \multicolumn{3}{c}{ African with Madagascar } \\
\hline $\mathbf{G}_{\text {st }}$ & $\mathbf{H}_{\mathbf{s}}$ & $\mathbf{H}_{\mathbf{t}}$ \\
\hline 0.01144 & 0.77841 & 0.78742 \\
0.00893 & 0.85366 & 0.86134 \\
0.01014 & 0.75987 & 0.76765 \\
0.01396 & 0.77162 & 0.78255 \\
0.00975 & 0.72939 & 0.73657 \\
0.00805 & 0.73330 & 0.73925 \\
0.01121 & 0.72826 & 0.73652 \\
0.00935 & 0.78460 & 0.79200 \\
0.00907 & 0.88447 & 0.89256 \\
0.01084 & 0.82766 & 0.83673 \\
0.00808 & 0.81412 & 0.82075 \\
0.01833 & 0.76488 & 0.77916 \\
0.00776 & 0.86543 & 0.87220 \\
0.00910 & 0.75812 & 0.76508 \\
0.00761 & 0.87767 & 0.88440 \\
0.01018 & 0.79543 & 0.80361
\end{tabular}

\begin{tabular}{ccc}
\hline \multicolumn{3}{c}{ Asian } \\
\hline $\mathbf{G}_{\text {st }}$ & $\mathbf{H}_{\mathbf{s}}$ & $\mathbf{H}_{\mathbf{t}}$ \\
\hline 0.00762 & 0.84274 & 0.84921 \\
0.00872 & 0.81684 & 0.82403 \\
0.00944 & 0.75890 & 0.76614 \\
0.00874 & 0.72284 & 0.72921 \\
0.01190 & 0.71940 & 0.72807 \\
0.01458 & 0.69915 & 0.70949 \\
0.00607 & 0.80012 & 0.80501 \\
0.00959 & 0.77473 & 0.78223 \\
0.00707 & 0.86775 & 0.87392 \\
0.01381 & 0.79937 & 0.81056 \\
0.01370 & 0.79304 & 0.80405 \\
0.00569 & 0.62611 & 0.62969 \\
0.00741 & 0.85317 & 0.85954 \\
0.00760 & 0.78443 & 0.79044 \\
0.00466 & 0.85686 & 0.86087 \\
0.00905 & 0.78103 & 0.78816 \\
\hline
\end{tabular}

\section{Supplementary Table 4: Total, inter- and intra-population genetic variance}




\begin{tabular}{|c|c|c|c|c|c|c|c|c|c|}
\hline \multirow[b]{2}{*}{ Locus } & \multicolumn{3}{|c|}{ Latin American } & \multicolumn{3}{|c|}{ European } & \multicolumn{3}{|c|}{ All populations } \\
\hline & $\mathbf{G}_{\text {st }}$ & $\mathbf{H}_{\mathrm{s}}$ & $\mathbf{H}_{\mathrm{t}}$ & $\mathbf{G}_{\mathbf{s t}}$ & $\mathbf{H}_{\mathrm{s}}$ & $\mathbf{H}_{\mathbf{t}}$ & $\mathbf{G}_{\text {st }}$ & $\mathbf{H}_{\mathrm{s}}$ & $\mathbf{H}_{\mathbf{t}}$ \\
\hline D8S1179 & 0.00533 & 0.79687 & 0.80114 & 0.00479 & 0.80173 & 0.80559 & 0.02000 & 0.80171 & 0.81808 \\
\hline D21S11 & 0.00407 & 0.83425 & 0.83765 & 0.00229 & 0.84684 & 0.84878 & 0.01964 & 0.83904 & 0.85585 \\
\hline D7S820 & 0.00753 & 0.79103 & 0.79703 & 0.00386 & 0.80061 & 0.80371 & 0.02224 & 0.77715 & 0.79482 \\
\hline CSF1PO & 0.00330 & 0.71758 & 0.71996 & 0.00606 & 0.72614 & 0.73057 & 0.01487 & 0.74070 & 0.75188 \\
\hline D3S1358 & 0.01200 & 0.75931 & 0.76853 & 0.00304 & 0.79534 & 0.79776 & 0.01611 & 0.74906 & 0.76132 \\
\hline TH01 & 0.01458 & 0.77061 & 0.78201 & 0.00865 & 0.77137 & 0.77810 & 0.05989 & 0.74425 & 0.79166 \\
\hline D13S317 & 0.01072 & 0.80817 & 0.81693 & 0.00297 & 0.77649 & 0.77881 & 0.03620 & 0.77090 & 0.79986 \\
\hline D16S539 & 0.00764 & 0.77955 & 0.78555 & 0.00175 & 0.75457 & 0.75590 & 0.01883 & 0.77702 & 0.79193 \\
\hline D2S1338 & 0.00894 & 0.86449 & 0.87229 & 0.00381 & 0.87090 & 0.87423 & 0.01925 & 0.87400 & 0.89115 \\
\hline D19S433 & 0.00778 & 0.82664 & 0.83312 & 0.00299 & 0.76087 & 0.76316 & 0.02152 & 0.81134 & 0.82919 \\
\hline vWA & 0.00751 & 0.79042 & 0.79640 & 0.00419 & 0.80765 & 0.81104 & 0.02298 & 0.80244 & 0.82132 \\
\hline TPOX & 0.00926 & 0.67460 & 0.68091 & 0.00561 & 0.61168 & 0.61513 & 0.03559 & 0.68819 & 0.71358 \\
\hline D18S51 & 0.00356 & 0.87685 & 0.87998 & 0.00474 & 0.87157 & 0.87572 & 0.01745 & 0.86831 & 0.88373 \\
\hline D5S818 & 0.01213 & 0.71663 & 0.72543 & 0.00421 & 0.71256 & 0.71557 & 0.02878 & 0.74418 & 0.76622 \\
\hline FGA & 0.00614 & 0.87368 & 0.87908 & 0.00375 & 0.85336 & 0.85657 & 0.01094 & 0.86791 & 0.87752 \\
\hline All Loci & 0.00796 & 0.79204 & 0.79840 & 0.00414 & 0.78411 & 0.78737 & 0.02403 & 0.79041 & 0.80987 \\
\hline
\end{tabular}

Supplementary Table 4: Total, inter- and intra-population genetic variance (Cont'd) 


\begin{tabular}{|c|c|c|c|c|c|c|c|c|c|c|c|c|c|c|c|c|}
\hline & US1 & US2 & ANG & SAF & EGU & HUT & KEN & MAD & MOZ & NWP & TUT & $\mathrm{CCS}$ & JAP & KOR & MAL & PHI \\
\hline US1 & & 231.37 & 274.24 & 401.03 & 231.16 & 290.16 & 202.69 & 351.59 & 372.76 & 211.53 & 487.75 & 1144.96 & 1917.27 & 1707.98 & 1149.36 & 953.06 \\
\hline US2 & 0.0524 & & 294.30 & 404.33 & 244.94 & 342.16 & 217.64 & 390.81 & 338.15 & 260.99 & 524.11 & 1354.70 & 2606.87 & 2136.76 & 1480.48 & 1149.23 \\
\hline ANG & 0.0000 & 0.0000 & & 315.24 & 224.30 & 266.23 & 202.20 & 380.37 & 343.92 & 295.92 & 391.04 & 1152.36 & 1814.77 & 1641.26 & 1227.65 & 987.45 \\
\hline SAF & 0.0000 & 0.0000 & 0.0000 & & 335.73 & 358.40 & 245.45 & 395.38 & 250.34 & 419.22 & 447.51 & 1244.97 & 1880.16 & 1711.28 & 1309.26 & 1095.10 \\
\hline EGU & 0.0302 & 0.0103 & 0.0169 & 0.0000 & & 299.34 & 194.76 & 372.47 & 320.27 & 239.05 & 413.61 & 1233.01 & 1999.51 & 1835.54 & 1268.44 & 1041.81 \\
\hline HUT & 0.0000 & 0.0000 & 0.0000 & 0.0000 & 0.0000 & & 210.35 & 384.45 & 358.15 & 344.63 & 363.93 & 1131.69 & 1652.72 & 1521.73 & 1140.98 & 1007.69 \\
\hline KEN & 0.1020 & 0.0247 & 0.0648 & 0.0001 & 0.1681 & 0.0507 & & 312.61 & 225.57 & 213.95 & 294.42 & 857.00 & 1168.80 & 1221.49 & 888.55 & 782.11 \\
\hline MAD & 0.0000 & 0.0000 & 0.0000 & 0.0000 & 0.0000 & 0.0000 & 0.0000 & & 379.17 & 431.85 & 473.88 & 697.44 & 907.37 & 947.66 & 638.76 & 530.65 \\
\hline MOZ & 0.0000 & 0.0000 & 0.0000 & 0.0003 & 0.0000 & 0.0000 & 0.0033 & 0.0000 & & 410.61 & 532.59 & 1345.85 & 2081.23 & 1937.59 & 1467.47 & 1167.28 \\
\hline NWP & 0.3446 & 0.0136 & 0.0000 & 0.0000 & 0.0423 & 0.0000 & 0.0757 & 0.0000 & 0.0000 & & 489.02 & 1441.89 & 2566.75 & 2199.37 & 1510.60 & 1223.42 \\
\hline TUT & 0.0440 & 0.0000 & 0.0000 & 0.0000 & 0.0000 & 0.0000 & 0.0000 & 0.0000 & 0.0000 & 0.0000 & & 1423.28 & 2103.06 & 1934.98 & 1492.11 & 1174.46 \\
\hline CCS & 0.0000 & 0.0000 & 0.0000 & 0.0000 & 0.0000 & 0.0000 & 0.0000 & 0.0000 & 0.0000 & 0.0000 & 0.0000 & & 418.85 & 420.65 & 394.87 & 339.75 \\
\hline JAP & 0.0000 & 0.0000 & 0.0000 & 0.0000 & 0.0000 & 0.0000 & 0.0000 & 0.0000 & 0.0000 & 0.0000 & 0.0000 & 0.0000 & & 501.19 & 795.81 & 511.80 \\
\hline KOR & 0.0000 & 0.0000 & 0.0000 & 0.0000 & 0.0000 & 0.0000 & 0.0000 & 0.0000 & 0.0000 & 0.0000 & 0.0000 & 0.0000 & 0.0000 & & 759.62 & 523.08 \\
\hline MAL & 0.0000 & 0.0000 & 0.0000 & 0.0000 & 0.0000 & 0.0000 & 0.0000 & 0.0000 & 0.0000 & 0.0000 & 0.0000 & 0.0000 & 0.0000 & 0.0000 & & 286.89 \\
\hline PHI & 0.0000 & 0.0000 & 0.0000 & 0.0000 & 0.0000 & 0.0000 & 0.0000 & 0.0000 & 0.0000 & 0.0000 & 0.0000 & 0.0000 & 0.0000 & 0.0000 & 0.0000 & \\
\hline MEX & 0.0000 & 0.0000 & 0.0000 & 0.0000 & 0.0000 & 0.0000 & 0.0000 & 0.0000 & 0.0000 & 0.0000 & 0.0000 & 0.0000 & 0.0000 & 0.0000 & 0.0000 & 0.0000 \\
\hline CSR & 0.0000 & 0.0000 & 0.0000 & 0.0000 & 0.0000 & 0.0000 & 0.0000 & 0.0000 & 0.0000 & 0.0000 & 0.0000 & 0.0000 & 0.0000 & 0.0000 & 0.0000 & 0.0000 \\
\hline HIS & 0.0000 & 0.0000 & 0.0000 & 0.0000 & 0.0000 & 0.0000 & 0.0000 & 0.0000 & 0.0000 & 0.0000 & 0.0000 & 0.0000 & 0.0000 & 0.0000 & 0.0000 & 0.0000 \\
\hline MAR & 0.0000 & 0.0000 & 0.0000 & 0.0000 & 0.0000 & 0.0000 & 0.0000 & 0.0000 & 0.0000 & 0.0000 & 0.0000 & 0.0000 & 0.0000 & 0.0000 & 0.0000 & 0.0000 \\
\hline MET & 0.0000 & 0.0000 & 0.0000 & 0.0000 & 0.0000 & 0.0000 & 0.0000 & 0.0000 & 0.0000 & 0.0000 & 0.0000 & 0.0000 & 0.0000 & 0.0000 & 0.0000 & 0.0000 \\
\hline PRA & 0.0000 & 0.0000 & 0.0000 & 0.0000 & 0.0000 & 0.0000 & 0.0000 & 0.0000 & 0.0000 & 0.0000 & 0.0000 & 0.0000 & 0.0000 & 0.0000 & 0.0000 & 0.0000 \\
\hline BRA & 0.0000 & 0.0000 & 0.0000 & 0.0000 & 0.0000 & 0.0000 & 0.0000 & 0.0000 & 0.0000 & 0.0000 & 0.0000 & 0.0000 & 0.0000 & 0.0000 & 0.0000 & 0.0000 \\
\hline SPA & 0.0000 & 0.0000 & 0.0000 & 0.0000 & 0.0000 & 0.0000 & 0.0000 & 0.0000 & 0.0000 & 0.0000 & 0.0000 & 0.0000 & 0.0000 & 0.0000 & 0.0000 & 0.0000 \\
\hline BLR & 0.0000 & 0.0000 & 0.0000 & 0.0000 & 0.0000 & 0.0000 & 0.0000 & 0.0000 & 0.0000 & 0.0000 & 0.0000 & 0.0000 & 0.0000 & 0.0000 & 0.0000 & 0.0000 \\
\hline CAU & 0.0000 & 0.0000 & 0.0000 & 0.0000 & 0.0000 & 0.0000 & 0.0000 & 0.0000 & 0.0000 & 0.0000 & 0.0000 & 0.0000 & 0.0000 & 0.0000 & 0.0000 & 0.0000 \\
\hline FLE & 0.0000 & 0.0000 & 0.0000 & 0.0000 & 0.0000 & 0.0000 & 0.0000 & 0.0000 & 0.0000 & 0.0000 & 0.0000 & 0.0000 & 0.0000 & 0.0000 & 0.0000 & 0.0000 \\
\hline POL & 0.0000 & 0.0000 & 0.0000 & 0.0000 & 0.0000 & 0.0000 & 0.0000 & 0.0000 & 0.0000 & 0.0000 & 0.0000 & 0.0000 & 0.0000 & 0.0000 & 0.0000 & 0.0000 \\
\hline
\end{tabular}

Refer to Table 1 for key to abbreviations.

Pair-wise population comparisons yielding statistically insignificant differences both before (in italics) and after (in bold) applying the Bonferroni correction.

Supplementary Table 5: G-test 


\begin{tabular}{|c|c|c|c|c|c|c|c|c|c|c|c|c|c|c|}
\hline & CSH & CAR & MEX & CSR & HIS & MAR & MET & PRA & BRA & SPA & BLR & CAU & FLE & POL \\
\hline US1 & 1581.20 & 720.66 & 1018.05 & 1093.93 & 681.27 & 717.26 & 1314.02 & 618.46 & 491.73 & 681.61 & 949.85 & 1044.45 & 1018.15 & 1360.81 \\
\hline US2 & 1991.02 & 924.57 & 1335.51 & 1557.23 & 863.07 & 943.29 & 1650.64 & 830.74 & 664.20 & 873.97 & 1193.36 & 1413.50 & 1345.87 & 1652.15 \\
\hline ANG & 1532.26 & 890.91 & 1168.84 & 1277.39 & 852.96 & 902.47 & 1414.07 & 779.70 & 646.26 & 879.73 & 1113.61 & 1268.78 & 1225.88 & 1283.22 \\
\hline EGU & 1666.15 & 853.74 & 1166.74 & 1308.00 & 842.06 & 892.42 & 1452.48 & 754.50 & 643.90 & 831.19 & 1117.48 & 1275.58 & 1177.84 & 1533.86 \\
\hline HUT & 1434.93 & 803.44 & 1042.83 & 1128.60 & 805.63 & 805.24 & 1316.93 & 691.05 & 639.72 & 803.92 & 982.72 & 1098.88 & 1081.38 & 1278.09 \\
\hline KEN & 1083.50 & 577.64 & 754.55 & 761.60 & 576.37 & 587.27 & 931.81 & 535.91 & 423.91 & 619.43 & 823.00 & 835.15 & 827.17 & 1003.52 \\
\hline MOZ & 1793.59 & 994.20 & 1349.94 & 1433.90 & 957.00 & 1030.06 & 1574.63 & 910.20 & 825.18 & 974.17 & 1309.76 & 1449.83 & 1426.98 & 1794.12 \\
\hline NWP & 2001.73 & 916.26 & 1304.43 & 1514.85 & 834.96 & 918.82 & 1625.91 & 753.80 & 647.19 & 871.08 & 1150.30 & 1319.93 & 1277.35 & 1601.97 \\
\hline TUT & 1824.54 & 1086.84 & 1376.41 & 1570.52 & 1021.23 & 1059.94 & 1574.57 & 992.01 & 926.14 & 987.93 & 1262.52 & 1511.79 & 1429.41 & 1530.42 \\
\hline $\mathrm{CCS}$ & 293.10 & 921.84 & 845.27 & 1095.84 & 676.42 & 815.87 & 1042.22 & 880.48 & 815.87 & 760.04 & 1017.96 & 1127.43 & 1100.27 & 1394.66 \\
\hline JAP & 584.83 & 1565.68 & 1607.56 & 1694.37 & 1016.61 & 1317.08 & 1656.62 & 1523.31 & 2001.13 & 1069.78 & 1627.64 & 2085.00 & 1912.00 & 2254.12 \\
\hline KOR & 559.27 & 1384.09 & 1368.58 & 1747.26 & 1004.39 & 1235.32 & 1540.45 & 1299.03 & 1632.93 & 1028.00 & 1376.46 & 1605.97 & 1542.45 & 1654.50 \\
\hline MEX & 0.0000 & 0.0000 & & 590.90 & 261.76 & 378.39 & 583.88 & 574.17 & 676.46 & 493.61 & 726.54 & 783.96 & 740.42 & 1136.39 \\
\hline CSR & 0.0000 & 0.0000 & 0.0000 & & 260.49 & 336.72 & 828.49 & 558.15 & 627.05 & 512.32 & 762.56 & 789.12 & 816.17 & 1130.95 \\
\hline HIS & 0.0000 & 0.1027 & 0.0025 & 0.0000 & & 220.76 & 442.05 & 304.36 & 336.70 & 378.29 & 507.03 & 451.80 & 484.11 & 479.11 \\
\hline MAR & 0.0000 & 0.4600 & 0.0000 & 0.0000 & 0.0106 & & 611.75 & 369.83 & 321.21 & 303.97 & 479.34 & 423.37 & 457.74 & 644.09 \\
\hline MET & 0.0000 & 0.0000 & 0.0000 & 0.0000 & 0.0000 & 0.0000 & & 941.78 & 1122.83 & 910.93 & 1208.44 & 1256.30 & 1165.76 & 1419.81 \\
\hline PRA & 0.0000 & 0.0000 & 0.0000 & 0.0000 & 0.0000 & 0.0000 & 0.0000 & & 338.29 & 376.59 & 593.38 & 509.84 & 543.58 & 481.19 \\
\hline BRA & 0.0000 & 0.0000 & 0.0000 & 0.0000 & 0.0000 & 0.0000 & 0.0000 & 0.0000 & & 365.67 & 556.06 & 508.96 & 548.30 & 469.52 \\
\hline SPA & 0.0000 & 0.0000 & 0.0000 & 0.0000 & 0.0000 & 0.0000 & 0.0000 & 0.0000 & 0.0000 & & 259.40 & 254.35 & 240.61 & 283.53 \\
\hline BLR & 0.0000 & 0.0000 & 0.0000 & 0.0000 & 0.0000 & 0.0000 & 0.0000 & 0.0000 & 0.0000 & 0.0000 & & 286.89 & 272.83 & 206.69 \\
\hline CAU & 0.0000 & 0.0000 & 0.0000 & 0.0000 & 0.0000 & 0.0000 & 0.0000 & 0.0000 & 0.0000 & 0.0000 & 0.0000 & & 229.52 & 265.03 \\
\hline FLE & 0.0000 & 0.0000 & 0.0000 & 0.0000 & 0.0000 & 0.0000 & 0.0000 & 0.0000 & 0.0000 & 0.0000 & 0.0000 & 0.0030 & & 340.50 \\
\hline
\end{tabular}

Refer to Table 1 for key to abbreviations.

Pair-wise population comparisons yielding statistically insignificant differences both before (in italics) and after (in bold) applying the Bonferroni correction.

\section{Supplementary Table 5: G-test (Cont'd)}




\section{THE GENETIC STRUCTURE OF POPULATIONS FROM HAITI AND JAMAICA REFLECT DIVERGENT DEMOGRAPHIC HISTORIES}

\section{A. Introduction}

The West Indian archipelago is bordered on the north by the southeastern United States, on the west by Central America, and on the south by the northern coast of South America. This island chain is classified into two groups: the Greater (northern) and Lesser (southeastern) Antilles, the two encompassing a broad arc 4,000 km long (Watts, 1987). The Antilles was first settled by Amerindians about 2,500-3,000 years before the present (YBP) (Keegan, 1995) who then served as slave laborers for the Spanish Crown. Overwork, malnourishment, and exposure to European diseases, concomitantly, led to the drastic reduction of the native population and consequently, a major deficit in the workforce (Rogoziński, 1999). As a result, enslaved migrants from the West African coast were imported into the New World beginning from the early 1500 s until the late 1800s (Knight and Crahan, 1979). At the same time, struggles ensued among the European powerhouses, namely Britain, Spain, France, and the Netherlands, for control of the West Indian islands. Because of the different European influences, the islands have experienced diverging cultural, economical, political, and demographic histories. In this study, I attempt to characterize the genetic input from European, African, and East Asian colonizations into the Greater Antillean islands of Haiti and Jamaica.

Haiti was the largest and most profitable of the French colonies in the New World during the 18th century, monopolizing the world's sugar $(40 \%)$ and coffee $(>50 \%)$ production (Knight, 2000). The rise in prosperity was accompanied by the importation of 
an estimated 860,000 African slaves into the island (Curtin, 1975; Coupeau, 2008) and, as a direct consequence, the number of Affranchis or gens de couleor (free coloreds and mulattoes) increased (Coupeau, 2008). Slave rebellions culminated in the Haitian Revolution (1789-1804) and ultimately resulted in the emigration of large numbers of French colonists and their slaves (Pamphile, 2001; Treco, 2002), which reduced the population to half of its original size (Ott, 1973). In 1804, Haiti was declared a republic (Knight, 2000; Coupeau, 2008), and under the newly established constitutions of the “world's first Black Republic" (Heinl and Heinl, 2005), whites were prohibited from purchasing land in the island until the American occupation (1915-1934) (Coupeau, 2008). Consequently, a schism was generated between the European and African populations, most likely resulting in endogamy, i.e., marriage within one's own race.

During the early 19th century, Jamaica surpassed Haiti as the principal sugarproducing island in the Antilles (Rogoziński, 1999). Along with this affluence came increased demands for plantation laborers that were satisfied originally by European (Irish, Scottish, and Welsh) white bond servants and later on, by the introduction of approximately 914,902 African slaves (Pepin, 2005) who, by the early 1800s, outnumbered the Caucasians 20:1 (Mordecai and Mordecai, 2001). According to Pepin (2005), 90.4\% of the total slave population in Jamaica disembarked from West African ports while the remaining 9.6\% were transported from Southeast Africa (Mozambique, Madagascar, and Tanzania) although a smaller proportion [7.7\% (Thomas, 1998; Pereira et al., 2001)] from the same region has been reported for the New World. Specifically, the Madagass, those native to Madagascar, were known to be present in Jamaica (Henriques, 1964). With the abolition of slavery and the culmination of the 
Apprenticeship period (1834-1838) throughout the British West Indies, massive labor shortages arose again, motivating the Jamaican government to contract more than 4,000 West European [British and German] and approximately 1,000 Chinese indentured servants to work on plantations between 1838 and 1918 (Lai, 1990, 1998). In addition to these two groups, a limited number of African laborers from Sierra Leone and Central Africa was also recruited (Roberts, 1954; Mordecai and Mordecai, 2001). The extent to which the aforementioned groups contributed to the current population's gene pool is uncertain since many returned to their native land upon expiration of their contracts (Roberts, 1954; Lai, 1990, 1998).

West African influences have become ingrained into both Haitian and Jamaican societies because of the large proportion of enslaved Africans transported from continental Africa to these New World destinations. The Yoruba speakers (natives of Nigeria and Benin), although not the most prominent group, seem to have had the greatest impact on Haitian society. The most practiced Yoruba customs include Haitian Vodou, an amalgamation of the religious beliefs of the Dahomey (present-day Benin) people and Roman Catholics (de Heusch, 1995; Brown, 1997), and its associated festival, Rara (Tselos, 1996). Yoruba imprints have also been detected in Haitian art (Okediji, 2003; Roberts, 2004), music, dance (Tselos, 1996) and folklore (Roberts, 2004). Jamaica, on the other hand, has not been impacted to such an extent by one particular ethnic group, but instead displays influences from various West African sources including the Akan (Mordecai and Mordecai, 2001), Coromantee, Ibo, Fula, Mandingo, and Yoruba peoples (Mason, 2000). Important cultural traditions derived from West Africa include obeah [magic] (Curtin, 1955), myalism [religion] (Curtin, 1955; Schuler, 1979), Jonkonnu 
[music and dance] (Curtin, 1955; Bettelheim, 1979), “jerk,” [a method of food preparation] and elements of the Jamaican dialect (Mordecai and Mordecai, 2001).

Studies delineating the genetic ancestry of the Haitian populace are limited. On the basis of the D1S80 polymorphic locus, Haiti was found to cluster with the continental African groups in both principal component (PC) and maximum likelihood (ML) analyses of worldwide populations (Herrera et al., 2004). In addition, using the polymarker system (PM) [lipoprotein receptor (LDLR), glycophorin A (GYPA), hemoglobin G gamma globin (HBGG), group specific component (GC), and D7S8], genetic affinities between Haiti and other African derived New World populations (French Antilles, Costa Rica, and Brazilian Mulattoes) were observed in a neighborjoining (NJ) tree (Hidalgo et al., 2005). Haitian women were also subjects in a clinical study examining the frequency of Duffy antigen receptor for chemokines (DARC) singlenucleotide polymorphisms (SNPs) in populations from the Caribbean, Europe, and the US. Seventy-five percent of the Haitian samples examined exhibited the CC genotype (absent among women of European ancestry) at levels comparable to females of African descent from the US (73\%) (Grann et al., 2008).

In contrast to Haiti, the genetic structure of the Jamaican population has been better characterized. In the aforementioned study by Grann et al. (2008), the frequency of the CC genotype among Jamaican females $(63 \%)$ is less than the levels present in either Haitian (75\%) or US African American (73\%) women. Close genetic ties between Jamaicans and continental Africans have been detected through analysis of $\beta$-globin gene polymorphisms (Antonarakis et al., 1984), autosomal SNP and insertion/deletion (INDEL) frequency data (Parra et al., 1998), mtDNA (Parra et al., 1998; McLean et al., 
2003, 2005), Y-chromosome markers (Bergen et al., 1999; Benn Torres et al., 2007), as well as 28 ancestry informative markers (AIMs) generated from autosomal SNP and INDEL loci (Benn-Torres et al., 2008). African genetic influences detected in Jamaica include the insertion allele in the Y-chromosome Alu polymorphism (YAP), the marker defining Y-haplogroups D-M174 and E-M96 (sub-Saharan African-specific), and the presence of mtDNA haplogroup L, prevalent throughout sub-Saharan Africa (Parra et al., 1998). European genetic contributions, on the other hand, are generally much lower, ranging from virtually no genetic influence (McLean et al., 2003) up to $18.1 \%$ (Gibbs et al., 1972) and vary depending on the marker system used (Parra et al., 1998; McLean et al., 2005; Benn-Torres et al., 2008). Furthermore, in addition to the presence of DNA from these two sources, Benn-Torres and colleagues (2008) have also reported limited Native American contributions (3.2\%) in Jamaica.

Reports surveying the genetic diversity present throughout the remainder of the West Indian archipelago also indicate strong genetic binds with continental Africans, although varying degrees of genetic input from other sources have been observed. In the Greater Antilles, for example, the Cuban population was found to share the majority (45.3\%) of its maternal component, but only a limited portion (19.7\%) of its Ychromosome lineages, with African sources (Mendizabal et al., 2008). Also, in an earlier study, in which HLA class I polymorphisms were examined, genetic contributions from Africa were detected in Cuban-Mulattoes at a comparable level (45.32\%) while the frequency in Cuban-Whites (21.46\%) was generally much lower (Ferrer et al., 2007). In addition, admixture estimates for the Puerto Rican population reveal signals of gene flow from mainland Africa [26\% when JC virus strains were examined (Fernández-Cobo et al., 
2001) and 27.2\% based on mtDNA haplogroup data (Martínez-Cruzado et al., 2005)], but in contrast to Cuba, Native Americans serve as the major contributor to this island's maternal gene pool.

Admixture profiles published for the Lesser Antillean populations also indicate significant gene flow from the African continent into the region. When Y-chromosome markers are used, collections from Grenada, St. Kitts, St. Vincent, St. Thomas, and Trinidad were all found to receive greater than $75 \%$ of their paternal contributions from African sources, whereas lower proportions were exhibited by the Dominican (34.1\%) and St. Lucian (45.8\%) samples. In the same group of populations, with the exception of St. Thomas, the mtDNA was also primarily African-specific (haplogroups L0, L1, L2, and L3), although Native American (A, C, and D, ranging from $0.63 \%$ to $2.87 \%$ ) and Eurasian $(\mathrm{H}, \mathrm{K}, \mathrm{M}$, and $\mathrm{N}$, ranging from $0.32 \%$ to $1.91 \%)$ haplogroups were detected at low frequencies (Benn Torres et al., 2007). Furthermore, in two additional studies, commensurate levels of African influences $(94 \%, 89.6 \%$, and $86.8 \%)$ were reported for Tobago (Miljkovic-Gacic et al., 2005), Barbados, and St. Thomas (Benn-Torres et al., 2008), respectively, when AIMs were employed. The authors also identify European and Native American influences at frequencies ranging from $4.6 \%$ to $10.6 \%$ and $0.2 \%$ to 2.6\%, respectively (Miljkovic-Gacic et al., 2005; Benn-Torres et al., 2008).

In the present study, allelic frequency data for 15 hypervariable autosomal STR loci are reported for the first time for the current populations of Haiti and Jamaica. Although the Jamaican populace has been examined previously across the 13 Combined DNA Index System (CODIS) STR loci (Budowle et al., 1999), the increased number of markers analyzed in this report allows for added resolution, thereby strengthening the 
statistical analyses. Phylogenetic comparisons to worldwide geographically targeted reference populations reveal strong genetic affinities between Haitians, Jamaicans, the other New World groups of African descent [New Providence (Bahamas) and African Americans from the US], and the continental African collections. Even though both Haiti and Jamaica exhibit their most substantial genetic input from Africa, Jamaica, in contrast to Haiti, also displays greater genetic contributions from Europe and East Asia.

\section{B. Materials and Methods}

\section{Sample collection and DNA extraction}

Buccal swabs, along with genealogical information for at least two generations, were collected at random from unrelated individuals belonging to the general populations of Haiti $(\mathrm{n}=111)$ and Jamaica $(\mathrm{n}=119)$. All ethical guidelines were followed as set forth by the Florida International University Institutional Review Board (IRB). DNA extractions were performed using the Gentra Buccal Cell Kit according to the manufacturer's specifications (Puregene, Gentra Systems, Minneapolis, MN) and stored at $-80^{\circ} \mathrm{C}$.

\section{Published data}

A total of 35 geographically targeted reference populations reporting 15 loci and 36 collections genotyped at 13 loci were included for comparison to the Haitian and Jamaican groups presented in this study. The African (East, West, and South) populations were chosen to assess their impact on Haiti and Jamaica, since both islands are known to 
have received genetic contributions from this region during slave trade and post-slave trade events. Inclusion of the Latin American collections is warranted because of the substantial African component detected by Simms et al. (2008). In addition, the East Asian collections were included to survey the extent to which this group influenced the Jamaican populace during the indentured labor system. All collections, their abbreviations, biogeographical origin, and average heterozygosity values are listed in Table 1.

DNA amplification and STR genotyping

The complete set of 15 autosomal STR loci (D8S1179, D21S11, D7S820, CSF1PO, D3S1358, TH01, D13S317, D16S539, D2S1338, D19S433, vWA, TPOX, D18S51, D5S818, and FGA) was amplified simultaneously using the AmpFlSTR Identifiler PCR Amplification Kit (Applied Biosystems, Foster City, CA) according to the manufacturer's instructions (Applied Biosystems, 2001). Amplified products were separated and detected by high performance multicapillary electrophoresis in an ABI 3130xl Genetic Analyzer with POP $7^{\mathrm{TM}}$ separation medium. ABI GeneScan ${ }^{\mathrm{TM}} 500 \mathrm{LIZ}^{\mathrm{TM}}$ was used as the internal size standard. The fragment analysis files generated were examined using the GeneMapper ${ }^{\circledR}$ software v3.2 which allows for sample comparisons to the commercially available allelic ladder and subsequent genotype assignment (Butler et al., 2004). 


\section{Statistical and phylogenetic analyses}

Allelic frequencies for the general populations of Haiti and Jamaica were estimated using the GenePop v 3.4 program (Raymond and Rousset, 1995). Using this data, histograms comparing the distribution of alleles in both collections per STR locus were constructed with Microsoft Excel. The Arlequin version 2.000 software (Schneider et al., 2000) was used to generate observed and expected heterozygosities $\left(\mathrm{H}_{\mathrm{o}}\right.$ and $\mathrm{H}_{\mathrm{e}}$, respectively) along with Hardy-Weinberg equilibrium (HWE) $P$ values and gene diversity indices (GDI). The Bonferroni adjustment $(\alpha=0.05 / 15=0.00333$ for 15 loci) was applied to minimize possible type I errors. In addition, to assess the effects of topogeographical barriers (e.g., the Caribbean Sea and the Atlantic Ocean) on gene flow and possible inbreeding in the three Afro-Caribbean collections, heterozygote deficiencies ( $\mathrm{F}_{\mathrm{IS}}$ and corresponding $P$ values) were computed for each of the 15 loci with GenePop v 3.4 (Raymond and Rousset, 1995).

Intra-, inter-, and total population $\left(\mathrm{H}_{\mathrm{s}}, \mathrm{G}_{\mathrm{st}}\right.$, and $\mathrm{H}_{\mathrm{t}}$, respectively) genetic variance values were calculated with the DISPAN program (Ota, 1993). The groups examined were assembled according to biogeographic origin as shown in Table 1 . Delta $(\delta)$ values for each of the 15 STR loci were also computed (Listman et al., 2007) for the grouped East versus West African collections to ascertain marker information content, i.e., the ability of the Identifiler loci to differentiate between the two groups of populations. Any locus yielding a $\delta$ value $\geq 0.30$ (Vergara et al., 2009) is considered informative for discriminating between the two sources. 
The Carmody's G-test program (Carmody, 1990) was used to assess the statistical significance of pair-wise population comparisons through 100,000 simulations, both before and after application of the Bonferroni correction $(\alpha=0.05 / 666=0.000075)$. Comparisons yielding $P$ values greater than $\alpha$ indicate genetic homogeneity among the populations.

Multidimensional scaling (MDS) plots (with associated stress values) were constructed with the statistical package SPSS version 14.0 (SPSS, 2001) using Nei's genetic distances $\left(F_{s t}\right)$ generated with the PHYLIP 3.52c software (Felsenstein, 2002). Phylogenetic comparisons of the Haitian and Jamaican collections with pertinent available reference populations were performed across the complete set of 15 loci and were repeated using 13 of the loci included in the set of 15 to allow inclusion of the Dominican Republic in the analysis. In addition, the New World (Haiti, Jamaica, New Providence, and the two US African American populations) and continental African groups [Madagascar was not included given its known Austronesian ancestry (Hurles et al., 2005; Regueiro et al., 2008)] were analyzed by themselves to better assess phylogenetic relationships among them. The aforementioned US African American collections, which represent a Minnesotan (US1) and a general population from the United States (US2), were included as additional representatives of New World Africans.

Admixture analyses were conducted to determine the genetic contributions made to the gene pools of the Haitian and Jamaican populations by three parental groups encompassing the geographical regions of Africa, East Asia and Europe [the Latin American collections were not included as a parental source because of the substantial proportion of their autosomal component shared with continental Africans and European 
(Simms et al., 2008)]. The Bahamian population of New Providence and the two US African American collections (US1 and US2) previously investigated by Simms et al. (2008) were also reexamined for their source populations in light of additional African data sets now available and employed in the present study.

To calculate admixture proportions $(\mathrm{m})$, three different approaches were taken. Using the SPSS version 14.0 software (SPSS, 2001), genetic contributions from the parental sources were generated using the weighted least squares (WLS) method proposed by Long et al. (1991). Admixture was also quantified with the ADMIX 2.0 program, which employs the coalescent-based approach described by Bertorelle and Excoffier (1998). Standard deviations were computed with bootstrapping implementing 1,000 reiterations. Additionally, with the LEADMIX program (Wang, 2003), admixture proportions were calculated using the least square regression method of Roberts and Hiorns (1965). Each of the aforementioned admixture estimators are referred to throughout the remainder of the article as $m_{\mathrm{L}}, m_{\mathrm{Y}}$, and $m_{\mathrm{RH}}$, respectively.

The admixture tests (Long et al., 1991) as well average heterozygosity calculations were repeated for the five New World African groups following the segregation of the 15 STR markers into slow ( $\geq 0.001$ mutations/meioses) vs. fast ( $\geq 0.01$ mutations/meioses) mutating loci. The analyses were performed to examine the effects of mutation rate on admixture proportions and genetic diversity indices. Mutation rates for the Identifiler loci were provided by the NIST STR database at http://www.cstl.nist.gov/div831/strbase/mutation.htm. 
The Structure software v. 2.3.1, available at

http://pritch.bsd.uchicago.edu/structure/html, was used to infer population substructure for both the Haitian and Jamaican collections (Pritchard et al., 2000). After uploading the genotypic data into the program, a length of burn-in period of 20,000 was selected and the number of Markov chain Monte Carlo (MCMC) repetitions after burn-in was also set to 20,000 . When updating the ancestry model specifications, the admixture model that performs clustering without using population of origin information and that assumes the allele frequencies in the population to be independent, was chosen. In the current study, the Structure analyses were performed with the number of ancestral population fixed at $K=3$ and $K=4$.

\section{Results}

Intra-population Diversity

Allelic frequencies for the 15 autosomal STR loci are listed in Tables 2 and 3 for Haiti and Jamaica, respectively, while $\mathrm{H}_{\mathrm{o}}, \mathrm{H}_{\mathrm{e}}$, HWE $P$ values and GDI for both populations are provided in Table 4. Histograms generated from the allele-frequency data generally reveal no differences in the distribution of alleles between the two collections but do illustrate variation in the frequencies of alleles across loci (Supporting Information Figure 1). In Jamaica, two loci (D16S539 and D2S1338) deviate from HWE expectations $(\alpha=0.05)$ but were found to be statistically insignificant after application of the Bonferroni adjustment. Interestingly, in the Haitian population, ten loci (D8S1179, D21S11, CSF1PO, D16S539, D2S1338, D19S433, vWA, TPOX, D5S818, and FGA) 
possess $\mathrm{H}_{\mathrm{o}}$ values exceeding $\mathrm{H}_{\mathrm{e}}$, whereas only three loci (D8S1179, D18S51, and FGA) in the Jamaican collection have higher than expected heterozygozities. Haiti and Jamaica both exhibit the highest genetic variability at locus D2S1338, with a GDI of 0.8887 and 0.8877, respectively, while D13S317, with values of 0.6717 for Haiti and 0.7132 for Jamaica, was found to be least variable in both populations. The combined power of exclusion (CPE) was $>99.9 \%$ for both collections; however, noticeable differences between the two populations were detected when comparing the combined power of discrimination (CPD), 0.733211 for Haiti versus 0.69732 for Jamaica, and average heterozygosities, 0.80300 versus 0.77647 , with Jamaica exhibiting the lower of the two values.

The number of alleles present in the Haitian population (160) and the Jamaican collection (158) outnumber the average allele count (149) for the continental African populations and are comparable in number to the other New World collections of African descent [New Providence (173), US African American 1 (162) and US African American 2 (168) (Simms et al., 2008)]. The Jamaican populace shares alleles D7S820-6 and FGA45.2 with Tanzania, whereas the Haitian collection possesses allele D16S539-6 characteristic of Angola. Both Haiti and Jamaica, along with New Providence, exhibit FGA-16.1 present in the East African nations of Madagascar and Tanzania. In addition, only two of the five New World African groups, Haiti and US African American 1 (US1), carry allele FGA-24.3, which is not present in any continental African collection examined in this report. Other alleles shared between these island populations and the mainland African groups include FGA-19.2 and 30.2. 
The highest intrapopulation variances $\left(\mathrm{H}_{\mathrm{s}}\right)$ are observed in the New World African populations (0.79889) and the Afro-Caribbean collections (0.79712), both of which exceed the values seen in the continental Africans including (0.79411) and excluding Madagascar (0.79356). The lowest $\mathrm{H}_{\mathrm{s}}$ levels are observed among the European (0.78461) and East Asian (0.78131) groups of populations (Table 5). Despite the highdiversity levels observed for the Afro-Caribbean group, $\mathrm{F}_{\text {IS }}$ calculations and associated $P$ values (Table 6) reveal two loci in Haiti (D7S820 and TH01), two loci in Jamaica (TH01 and D2S1338), and five loci in New Providence (D21S11, CSF1PO, D19S433, TPOX, and FGA) that exhibit lower than the statistically expected number of heterozygotes.

\section{Inter-population Diversity}

Phylogenetic relationships among all populations examined in this study were investigated using MDS projections, pair-wise population comparisons (G-test) and admixture analyses. In addition, $\mathrm{G}_{\mathrm{st}}$ and $\mathrm{H}_{\mathrm{t}}$ values were computed to assess inter- and total population variance, respectively, whereas $\delta$ values were calculated to evaluate the ability of the 15 Identifiler loci to statistically differentiate between the grouped East and West African populations.

In the MDS plot of all populations (Fig. 1A), three groupings according to biogeographical origin are evident: an East Asian cluster in the lower left quadrant, a Latin American/European assemblage in the upper left section (although a clear partitioning between the two is apparent), and an African group occupying the right half of the graph. At the periphery of the African cluster, Jamaica, along with the two US African-American collections (US1 and US2), New Providence and Angola, segregate 
away from the mainland African populations in the direction of the Latin American/European conglomerate. However, when only the African and African derived collections are employed (Fig. 1B), Jamaica plots on the outskirts of the central cluster at a considerable distance from Angola and the New World African populations. In contrast, Haiti maps among the continental Africans in both dendrograms (Fig. 1A,B) but lies closest to two of the West African collections (Guinea-Bissau and Equatorial Guinea) when all the populations are examined (Fig. 1A) and displays greater genetic affinities with Angola (Dimension 1), US2, and Equatorial Guinea (Dimension 2) when only the African and African derived groups are considered (Fig. 1B). At 13 loci, Haiti plots distantly from the general populace of the Dominican Republic (Supporting Information Figure 2), thereby suggesting a differential contribution of source populations as well as limited gene flow between the two portions of the island. Although the Latin American collections plot close to the European groups (Fig. 1A), they clearly segregate in two directions: the Hispanics from Minnesota in the direction of the East Asian cluster while the Brazilians and Puerto Rican Americans head toward the African collections. The tendency of some Latin American groups to segregate toward the East Asian collections is possibly the result of the Amerindian component in those New World populations. The stress value for the all population projection was 0.10202 , whereas estimates for the African and African derived groups alone led to an output of 0.19149 .

Inter- and total population diversity indices $\left(\mathrm{G}_{\mathrm{st}}\right.$ and $\mathrm{H}_{\mathrm{t}}$, respectively) are presented in Table 5. The highest $\mathrm{G}_{\mathrm{st}}$ values are recorded for the All populations group (0.02400) and are lowest among the New World African collections (0.00330), likely attributed to the genetic contributions made to the populations in the group by similar 
sources. Total population variance $\left(\mathrm{H}_{\mathrm{t}}\right)$ is also highest in the All populations assemblage (0.81014) and is followed in decreasing order by the Africans including $(0.80320)$ and excluding (0.80169) Madagascar, New World Africans (0.80154), Afro-Caribbeans (0.79984), Latin Americans (0.79840), East Asians (0.78807), and Europeans (0.78789).

Delta $(\delta)$ values calculated for the grouped East versus West African collections are provided in Table 7. The average $\delta$ across the entire set of 15 loci was 0.088 , and values at each locus for the pair ranged from 0.045 to 0.117 . Since no locus attained a value $\geq 0.30$, these markers are not considered informative for statistically differentiating between the two groups of populations.

In the G-test pair-wise analyses (Table 8), statistically insignificant genetic differences are observed between several of the New World and continental African collections, indicating a close genetic relationship between the two groups of populations. Specifically, all five New World African populations were not found to differ significantly from Kenya or Equatorial Guinea. G-test results also indicate genetic similarities between the Afro-Caribbean collections and Guinea-Bissau as well as between Haiti, Jamaica, US1, and Angola. Given the genetic affinities displayed by the New World and continental African collections, in combination with the large proportion of enslaved Africans transported to the New World, it is not surprising that results from the pair-wise population comparisons reflect genetic homogeneity between the AfroCaribbean and Afro-American collections.

Admixture estimates for the New World African collections employing grouped African, East Asian, and European populations as parentals are provided in Table 9. The results generated from each of the three methods used, as well as for the admixture tests 
involving the segregated loci (Supporting Information Table 1), collectively indicate that the most significant genetic contributions made to the gene pools of all five hybrid populations originate from Africans. In particular, $m_{\mathrm{L}}$ and $m_{\mathrm{RH}}$ estimates demonstrate that of all the New World collections, the Haitian populace receives the largest African genetic component, sharing greater than $95 \%$ of its DNA with this group, while comparable levels of genetic input (ranging from $69.7 \%$ to $83.7 \%$, depending on the approach taken to quantify admixture) from African sources are detected in the other hybrid populations. The remaining genetic influences observed in New World Africans, with the exception of New Providence, arise from both the European and East Asian collections. In Jamaica, genetic contributions from the European $\left(m_{\mathrm{L}}=16 \%, m_{\mathrm{Y}}=17.9 \%\right.$ and $\left.m_{\mathrm{RH}}=14.2 \%\right)$ and East Asian $\left(m_{\mathrm{L}}=5.7 \%, m_{\mathrm{Y}}=5.65 \%\right.$ and $\left.m_{\mathrm{RH}}=3.73 \%\right)$ sources are commensurate among the methods employed, whereas in Haiti and the two AfroAmerican populations, the degree of European and East Asian admixture varies according to the method employed. Interestingly, of the New World African groups, Jamaica is the only collection to exhibit significant variation in admixture proportions between slow and fast mutating loci.

The divergent genetic signals observed in the admixture profiles for Haiti and Jamaica are not reflected in the output of the Structure analyses. The bar plots generated for both groups (Supporting Information Figure 3), assuming either three $(K=3)$ or four $(K=4)$ ancestral populations, reveal no population substructure in either collection. The graphs also indicate that both groups receive equivalent contributions from the same ancestral source populations. The general lack of population substructure observed in Haiti and Jamaica is likely attributed to the limited number of loci (15) employed in the 
analyses (Listman et al., 2007), which according to Ardlie and colleagues (2002) should be much larger ( $>100)$ to reliably differentiate between highly admixed populations sharing close genetic ties.

\section{Discussion}

The Antilles is recognized as a "great melting pot," (Knight and Crahan, 1979) with influences that stem from Africa, Europe, and East Asia; however, the extent to which these ancestral source populations have contributed genetically to the island chain remains relatively unexplored. Thus, in this study, 15 autosomal STR loci were typed in the general populations of Haiti and Jamaica to assess the genetic implications of their known divergent colonial histories and associated migratory events. This investigation represents the first time that Haitian STR allelic frequency data are presented, whereas the Jamaican population is examined across the full set of 15 STR loci since D2S1338 and D19S433 were not previously reported (Budowle et al., 1999). Inclusion of additional loci in the dataset not only increases the phylogenetic resolution between closely related populations, but also generates more robust probability calculations important in forensic and/or paternity testing.

Genetic diversity in the New World is expected to be high because of genetic contributions from various founder populations and subsequent admixture events over the last 500 years. Historical accounts for the West Indies indicate extensive gene flow from continental Africa during the period of the Transatlantic slave trade in addition to varying degrees of genetic input from European, Asian, and/or other sources (Parra et al., 1998; Saunders, 2003; Herrera et al., 2004; Pepin, 2005; Benn Torres et al., 2007; Benn-Torres 
et al., 2008). For instance, Jamaica, whose motto is "Out of many one people," has been settled by a wide array of sources including enslaved Africans, Portuguese Jews, Scottish and Irish bond servants, German and Chinese indentured laborers, and later on Syrian (Henriques, 1964), Cuban and Haitian (Thomas-Hope, 2003) nationals, whereas Haiti, the "world's first Black Republic," has received gene flow, for the most part, exclusively from Africa. Given the high level of diversity anticipated for the region, it is not surprising that average heterozygosity values for all five New World collections (Table 1) are comparable to the combined average heterozygosity of the mainland African populations (0.79778) included in the present study. With respect to Haiti $(0.80300)$ and Jamaica $(0.77647)$, notable differences in average heterozygosities are observed for each collection upon segregation of the STR markers into slow versus fast mutating loci [ 0.74325 versus 0.66387 (slow) and 0.81200 versus 0.79380 (fast) for Haiti and Jamaica, respectively], but this disparity should be tempered considering the unequal distribution of loci within the two categories. The New World Africans also exhibit the highest intra-population variance level of any group examined (Table 5).

Although $\delta$ values generated for the 15 Identifiler loci (Table 7) do not support the segregation of the African collections into East and West, noticeable differences between the two groups of populations are observed in both the MDS analysis (Fig. 1B) and the G-test (Table 8). In the MDS plot of African and African derived populations, noteworthy is the partitioning of the East African collections. Although the East African collections from Mozambique, Tanzania, and Kenya were found to group with the West African (Angola, Equatorial Guinea, and Guinea-Bissau) and New World populations in the central cluster, the Uganda, Hutu, and Tutsi collections lie on the fridges of this 
assemblage. A plausible explanation for the lack of discrimination observed between the East and West African populations is the Bantu expansion, which is known to have been a venue for the demic diffusion of a number of West African markers, including Yhaplogroups E1b1a, E2, and B2a (Berniell-Lee et al., 2009), as well as mtDNA haplogroup L subclades (specifically L1a and L2a), into East Africa (Salas et al., 2002). Moreover, the Bantu diaspora is also likely the cause for genetic similarities observed in the G-test between Kenya and the West African groups (with the exception of Namibia). Admixture proportions generated for the five New World African populations (Table 9), regardless of the method employed, indicate that their strongest genetic signal derives from the African continent. Given the large number of African laborers [estimates ranging from about 6,000 (Dominican Republic) to greater than one million (Brazil) slaves (Pepin, 2005)] transported to the region during the period of the Transatlantic slave trade, these findings are not surprising. In Jamaica, the relatively high rate of the YAP insertion [ranging from 17.89\% (Parra et al., 1998) to 59.09\% (Benn Torres et al., 2007)] in combination with elevated levels of Y-haplogroup E1bla-M2 (about 43\%) (Athey, 2006; Benn Torres et al., 2007) and mtDNA haplogroup L (25.86\%) (Parra et al., 1998), corroborate the large African component detected in this population in the present study. Africa's prominent contribution to the Haitian gene pool is supported by an earlier D1S80 study in which Haiti was found to cluster with continental Africans in PC and ML analyses of worldwide populations (Herrera et al., 2004). In addition, phylogenetic assessments performed by Simms and colleagues (2008) reveal strong genetic affinities between New Providence in the Bahamas, the two US African American groups and the continental Africans. 
Of the five New World African groups, the percent contribution from continental Africa is greatest in Haiti for two of three admixture tests performed. The larger percentage of African DNA present in the Haitian gene pool is supported by Haiti's position amidst the African collections in the MDS plot of all populations (Fig. 1A). Within this cluster, Haiti maps in closest proximity to Guinea-Bissau and Equatorial Guinea in both Dimensions. Likewise, when only the African and African derived populations are considered, the Haitian populace still exhibits genetic affinities with the West Africans. In addition, pair-wise population comparisons (Table 8) performed also indicate genetic homogeneity between Haiti/Angola, Haiti/Equatorial Guinea, and Haiti/Guinea-Bissau. These findings are supported by a previous study, based on the D1S80 polymorphic locus, in which Haiti was found to map equidistant from Benin and a general collection from the Bahamas in the PC plot of African and New World African populations (Herrera et al., 2004).

Admixture data for the Jamaican collection reveals commensurate contributions from Africa (ranging from $76.5 \%$ to $82.1 \%$ ), regardless of the method employed to quantify admixture. Genetic ties with the African continent are not only observed in the clustering of Jamaica, along with the other New World African groups, on the fringes of the African assemblage in Figure 1A, but are also detected in the results of the pair-wise comparisons (Table 8), which indicate genetic similarities between Jamaica, the West African collections (Angola, Equatorial Guinea, and Guinea-Bissau), and Kenya. Within the assembly of New World populations (Fig. 1A), noteworthy is the position of Angola, which maps distantly from the remaining continental African groups. The close proximity of Jamaica and Angola in Figures 1A (Dimensions 1 and 2) and 1B (Dimension 2) most 
likely derives from the large proportion of enslaved Africans [approximately 142,725 persons according to Pepin (2005)] transported to the island from West-Central Africa, the region encompassing Angola and the Congos. Genetic influences in Jamaica from West Africa have also been detected in earlier studies in which elevated levels (approximately $70 \%$ ) of the Benin $\beta^{\mathrm{S}}$-haplotype, prevalent throughout West-Central Africa [Cameroon, Nigeria, and Benin), have been reported (Wainscoat et al., 1983; Antonarakis et al., 1984; Nagel et al., 1984; Pante-de-Sousa, 1998). Also, in a more recent study examining mtDNA haplotypes, the Jamaican population was found to branch together with samples native to Sierra Leone in NJ trees (McLean et al., 2005). This clustering is expected since indentured servants from this region of West Africa were transported to Jamaica during the postslavery period.

The comparable African admixture proportions exhibited by the New Providence and US African American collections (US1 and US2) echoes data published by Simms et al. (2008). However, with the introduction of additional African populations in the present study (Guinea-Bissau, Namibia, Tanzania, and Uganda), the contributions from Africa have decreased on average by $0.93 \%, 5.76 \%$, and $10.8 \%$ when the Roberts and Hirons (1965), Long (1991), and Bertorelle and Excoffier (1998) approaches were employed, respectively. Despite this decrease, genetic affinities between these collections and the continental African groups are still the most prominent.

The remaining influences present in each of the New World African population's gene pool are contributed by the European groups and, except for the New Providence collection, the East Asian populations as well. The genetic input from Europeans is minimal in Haiti (with the exception of the $m_{\mathrm{Y}}$ estimator) when compared with the other 
New World African populations that exhibit much larger contributions (ranging from $14.2 \%$ to $36.3 \%$ ). These findings are corroborated by the MDS plot of all populations (Fig. 1A), which illustrates the latter populations plotting closely together on the outskirts of the African assemblage but in the direction toward the European/Latin American group while the Haitian collection maps amidst the mainland African populations within the African cluster.

European gene flow into Jamaica is supported by previous studies which report admixture profiles portraying from as little as no apparent contributions up to $2 \%$ when mtDNA haplotypes were analyzed (McLean et al., 2003, 2005), 6.8\% when autosomal SNP and INDEL frequency distributions were employed (Parra et al., 1998), 12.4\% when a panel of 28 AIMs generated from SNP and INDEL frequency data was utilized (BennTorres et al., 2008) and 18.1\% when glucose-6-phosphate dehydrogenase (G6PD) deficiency alleles were examined (Gibbs et al., 1972). Genetic contributions from Europe most likely derive from the influx of British soldiers, white bond servants and, later, as a result of the immigration of Western European indentured laborers. In contrast to the other New World African collections, the admixture proportions for Haiti, with the exception of the coalescent based approach, indicate only minor contributions from European sources. The limited presence of European DNA in the current population is likely due to ramifications of the Haitian Revolution in which the French colonists were either killed or coerced out of the island.

Signals of East Asian gene flow into the Jamaican population most likely stem from the influx of indentured servants contracted from China between 1852 and 1884 to replace the dwindling African sources (Lai, 1990). Demographic data reports that the 
Chinese comprise $0.2 \%$ of the total Jamaican populace (Mordecai and Mordecai, 2001), supporting the minor East Asian component detected in the present study. Although less likely, genetic input from East Asia may have also arisen from remnants of the indigenous Arawak Indian population still present in Jamaica before the British occupation of the island (Henriques, 1964). Genetic imprints (SNP and INDEL AIMs) of this group have been reported by Benn-Torres and collaborators (2008) who observed minor Native American genetic influences (3.2\%) in the island. In contrast, Haiti exhibits minimal East Asian contributions (0.3\%) only when the Long (1991) method is employed. Genetic influences from this region may be attributed to residual Native American input or to gene flow from East Asian migrants ubiquitous to the New World.

Overall, my results substantiate greater genetic contributions to Haiti from Africa than to Jamaica, which shares larger portions of its autosomal component with the European and East Asian groups. The prominent presence of African DNA in the Haitian and Jamaican populations is likely the result of the transportation of hundreds of thousands of enslaved Africans to the colony from ports along Africa's West coast. My data clearly demonstrates that among the New World African collections, the contributions of different ancestral groups of populations, namely Africans, Europeans, and East Asians, varies and corroborates information from historical accounts of migratory events. 


\section{REFERENCES}

Alves C, Gusmão L, Damasceno A, Soares B, Amorim A. 2004. Contribution for an African autosomic STR database (AmpFISTR Identifiler and Powerplex 16 system) and a report on genotypic variations. Forensic Sci Int 139:201-205.

Alves C, Gusmão L, López-Parra AM, Soledad Mesa M, Amorim A, Arroyo-Pardo E. 2005. STR allelic frequencies for an African population sample (Equatorial Guinea) using AmpFISTR Identifiler and Powerplex 16 kits. Forensic Sci Int 148:239-242.

Antonarakis SE, Boehm CD, Serjeant GR, Theisen CE, Dover GJ, Kazazian HH. 1984. Origin of the $\beta^{\mathrm{S}}$-globin gene in Blacks: the contribution of recurrent mutation or gene conversion or both. Proc Natl Acad Sci 81:853-856.

Applied Biosystems. 2001. AmpFISTR Identifiler PCR amplification kit user's manual. Foster City, CA: Applied Biosystems.

Ardlie KG, Lunetta KL, Seielstad M. 2002. Testing for population subdivision and association in four case-control studies. Am J Hum Genet 71:304-311.

Athey W. 2006. Haplogroup prediction from Y-STR values using a Bayesian-AlleleFrequency approach. J Genet Geneal 2:34-39.

Beleza S, Alves C, Reis F, Amorim A, Carracedo A, Gusmão L. 2004. 17 STR data (AmpFlSTR Identifiler and Powerplex 16 system) from Cabinda (Angola). Forensic Sci Int 141:193-196.

Benn-Torres J, Bonilla C, Robbins CM, Waterman L, Moses TY, Hernandez W, Santos ER, Bennett F, Aiken W, Tullock T, Coard K, Hennis A, Wu S, Nemesure B, Leske MC, Freeman V, Carpten J, Kittles RA. 2008. Admixture and population stratification in African Caribbean populations. Ann Hum Genet 72:90-98.

Benn Torres J, Kittles RA, Stone AC. 2007. Mitochondrial and Y chromosome diversity in the English-Speaking Caribbean. Ann Hum Genet 71:1-9.

Bergen AW, Wang CY, Tsai J, Jefferson K, Dey C, Smith KD, Park SC, Tsai SJ, Goldman D. 1999. An Asian-Native American paternal lineage identified by RPS4Y resequencing and by microsatellite haplotyping. Ann Hum Genet 63:63-80.

Bernal LP, Borjas L, Zabala W, Portillo MG, Fernández E, Delgado W, Tovar F, Lander N, Chiurillo MA, Ramírez JL, García O. 2006. Genetic variation of 15 STR autosomal loci in the Maracaibo population from Venezuela. Forensic Sci Int 161:60-63. 
Berniell-Lee G, Calafell F, Bosch E, Heyer E, Sica L, Mouguiama-Daouda P, van der Veen L, Hombert J, Quintana-Murci L, Comas D. 2009. Genetic and demographic implications of the Bantu expansion: insights from human paternal lineages. Mol Biol Evol 26:1581-1589.

Bertorelle G, Excoffier L. 1998. Inferring admixture proportions from molecular data. Mol Biol Evol 15:1298-1311.

Bettelheim J. 1979. Jamaican Jonkonnu and related Caribbean festivals. In: Knight F, Crahan M, editors. Africa and the Caribbean. Baltimore, MA: The Johns Hopkins University Press. p 80-100.

Brown KM. 1997. Systematic remembering, systematic forgetting: Ogou in Haiti. In Barnes ST, editor. Africa's Ogun: Old world and new. Bloomington, IN: Indiana University Press. p 65-89.

Budowle B, Moretti T, Baumstark AL, Defenbaugh DA, Keys KM. 1999. Population data on the thirteen CODIS core short tandem repeat loci in African Americans. U.S. Caucasians, Hispanics, Bahamians, Jamaicans, and Trinidadians. J Forensic Sci 44:12771286.

Butler JM, Buel E, Crivellente F, McCord BR. 2004. Forensic DNA typing by capillary electrophoresis using the ABI Prism 310 and 3100 genetic analyzers for STR analysis. Electrophoresis 25:1397-1412.

Butler JM, Schoske R, Vallone PM, Redman JW, Kline MC. 2003. Allele frequencies for 15 autosomal STR loci on U.S. Caucasian, African American, and Hispanic populations. J Forensic Sci 48:908-911.

Camacho MV, Benito C, Figueiras AM. 2007. Allelic frequencies of the 15 STR loci included in the AmpFISTR ${ }^{\circledR}$ Identifiler $^{\mathrm{TM}}$ PCR Amplification kit in an autochthonous sample from Spain. Forensic Sci Int 173:241-245.

Carmody G. 1990. G-test. Ottowa, Canada: Carleton University.

Chiurillo MA, Morales A, Mendes AM, Lander N, Tovar F, Fuentes A, Ramírez JL. 2003. Genetic profiling of a central Venezuelan population using 15 STR markers that may be of forensic importance. Forensic Sci Int 136:99-101.

Coudray C, Calderon R, Guitard E, Ambrosio B, González-Martín A, Dugoujon J. 2007. Allele frequencies of 15 tetrameric short tandem repeats (STRs) in Andalusians from Huelva (Spain). Forensic Sci Int 168:e21-e24.

Coupeau S. 2008. The history of Haiti. Wesport, CT: Greenwood Press. 
Curtin PD. 1975. Economic change in pre-colonial Africa: Sengambia in the era of the slave trade. Madison, WI: The University of Wisconsin Press.

Curtin PD. 1955. Two Jamaicas: the role of ideas in a tropical colony 1830-1865. Cambridge, MA: Harvard University Press.

Czarny J, Grzybowski T, Derenko MV, Malyarchuk BA, Śliwka DM. 2005. Genetic variation of 15 STR loci (D3S1358, vWA, FGA, TH01, TPOX, CSF1PO, D5S818, D13S317, D75820, D16S539, D2S1338, D8S1179, D21S11, D18S51, and D19S433) in populations of north and central Poland. Forensic Sci Int 147:97-100.

de Heusch L. 1995. Kongo in Haiti: a new approach to religious syncretism. In: Davis DJ, editor. Slavery and beyond: the African impact on Latin America and the Caribbean. Lanham, MD: SR Books. p 103-120.

de Souza Góes AC, da Silva DA, Fonseca Gil EH, da Silva MTD, Pereira RW, de Carvalho EF. 2004. Allele frequencies data and statistic parameters for 16 STR loci D19S433, D2S1338, CSF1PO, D16S539, D7S820, D21S11, D18S51, D13S317, D5S818, FGA, Penta E, TH01, vWA, D8S1179, TPOX, D3S1358 - in the Rio de Janeiro population, Brazil. Forensic Sci Int 140:131-132.

De Ungria MCA, Roby RK, Tabbada KA, Rao-Coticone S, Tan MMM, Hernandez KN. 2005. Allele frequencies of 19 STR loci in a Philippine population generated using AmpFISTR multiplex and ALF singleplex systems. Forensic Sci Int 152:281-284.

Díaz V, Rivas P, Olivero C, Carracedo A. 2008. The distribution of allele frequencies of 15 STRs in Dominican population. Forensic Sci Int: Genet Supplement Series 1:322-325.

Felsenstein J. 2002. Phylogeny inference package (PHYLIP), Version 3.6a3. Seattle: Department of Genetics, University of Washington.

Fernández-Cobo M, Jobes DV, Yanagihara R, Nerurkar VR, Yamamura Y, Ryschkewitsch CF, Stoner GL. 2001. Reconstructing population history using JC virus: Amerinds, Spanish, and Africans in the ancestry of modern Puerto Ricans. Hum Biol 73:385-402.

Ferrer A, Nazábal M, Companioni O, Fernández de Cossío ME, Camacho H, Cintado A, Benítez J, Casalvilla R, Sautié M, Villareal A, Díaz T, Marrero A, Fernández de Cossío J, Hodelin A, Leal L, Ballester L, Novoa LI, Middleton D, Dueñas M. 2007. HLA class I polymorphism in the Cuban population. Hum Immunol 68:918-927.

Forward BW, Eastman MW, Nyambo TB, Ballard RE. 2008. AmpFISTR ${ }^{\circledR}$ Identifiler $^{\mathrm{TM}}$ STR allele frequencies in Tanzania, Africa. J Forensic Sci 53:245-247. 
Gibbs WN, Ottey F, Dyer H. 1972. Distribution of Glucose-6-Phosphate Dehydrogenase phenotypes in Jamaica. Am J Hum Genet 24:18-23.

Gorostiza A, González-Martín A, Ramírez CL, Sánchez C, Barrot C, Ortega M, Huguet E, Corbella J, Gené M. 2007. Allele frequencies of the 15 AmpFlSTR Identifiler loci in the population of Metztitlán (Estado de Hidalgo), México. Forensic Sci Int 166:230-232.

Grann VR, Ziv E, Joseph CK, Neugut AI, Wei Y, Jacobson JS, Horwitz MS, Bowman N, Beckmann K, Hershman DL. 2008. Duffy (Fy), DARC, and neutropenia among women from the United States, Europe and the Caribbean. Brit J Haematol 143:288-293.

Gross AM, Budowle B. 2006. Minnesota population data on 15 STR loci using the Identifiler ${ }^{\circledR}$ kit. J Forensic Sci 51:1410-1413.

Gusmão L, Sánchez-Diz P, Gomes I, Alves C, Carracedo Á,Prata MJ, Amorim A. 2006. Genetic analysis of autosomal and Y-specific STRs in the Karimojong population from Uganda. Int Congr Ser 1288:213-215.

Hashiyada M, Itakura Y, Nagashima T, Nata M, Funayama M. 2003. Polymorphism of 17 STRs by multiplex analysis in Japanese population. Forensic Sci Int 133:250-253.

Heinl RD, Heinl NG. 2005. Written in blood: the story of the Haitian people 1492-1995. Lanham, MD: University Press of America.

Henriques F. 1964. Jamaica: land of wood and water. New York, NY: London House \& Maxwell.

Hernández-Gutiérrez S, Hernández-Franco P, Martínez-Tripp S, Ramos-Kuri M, RangelVillalobos H. 2005. STR data for 15 loci in a population sample from the central region of Mexico. Forensic Sci Int 151:97-100.

Herrera RJ, Adrien LR, Ruiz LM, Sanabria NY, Duncan G. 2004. D1S80 single-locus discrimination among African populations. Hum Biol 76:87-108.

Hidalgo PC, Bengochea M, Abilleira D, Cabrera A, Alvarez I. 2005. Genetic admixture estimate in the Uruguayan population based on the loci LDLR, GYPA, HBGG, GC and D7S8. Int J Hum Genet 5:217-222.

Hu SP, Yu XJ, Liu JW, Cai KL. 2005. Analysis of STR polymorphisms in the Choa Shan population in South China. Forensic Sci Int 147:93-95.

Hurles ME, Sykes BC, Jobling MA, Forster P. 2005. The dual origin of the Malagasy in island Southeast Asia and East Africa: evidence from maternal and paternal lineages. Am J Hum Genet 76:894-901. 
Keegan WF. 1995. Modeling dispersal in the prehistoric West Indies. World Archaeol 26:400-420.

Kido A, Dobashi Y, Fujitani N, Hara M, Susukida R, Kimura H, Oya M. 2007. Population data on the AmpFISTR Identifiler loci in Africans and Europeans from South Africa. Forensic Sci Int 168:232-235.

Kim Y, Hwang J, Kim Y, Lee S, Chung N, Goh H, Kim C, Kim D. 2003. Allele frequencies of 15 STR loci using AmpFISTR Identifiler kit in a Korean population. Forensic Sci Int136:92-95.

Knight F. 2000. The Haitian Revolution. Am Hist Rev 105:103-115.

Knight F, Crahan M. 1979. The African migration and the origins of an Afro-American society and culture. In: Knight F, Crahan M, editors. Africa and the Caribbean. Baltimore, MA: The Johns Hopkins University Press. p 1-19.

Lai L. 1990. Chinese indentured labor migrations to the British West Indies in the nineteenth century. In Sanjek R, editor. Caribbean Asians: Chinese, Indian and Japanese experiences in Trinidad and the Dominican Republic. New York, NY: Queens College. p 3-30.

Lai L. 1998. The Chinese in the West Indies 1806-1995: a documentary history. Kingston, Jamaica: The Press University of the West Indies.

Listman JB, Malison RT, Sughondhabirom A, Yang B, Raaum RL, Thavichachart N, Sanichwankul K, Kranzler HR, Tangwonchai S, Mutirangura A, Disotell TR, Gelernter J. 2007. Demographic changes and marker properties affect detection of human population differentiation. BMC Genet 8:21.

Long JC, Williams RC, McAuley JE, Meids R, Partel R, Tregellas M, South SF, Rea AE, McCormick B, Iwaniec U. 1991. Genetic variation in Arizona Mexican Americans: estimation and interpretation of admixture proportions. Am J Phys Anthropol 84:141157.

Martínez-Cruzado JC, Toro-Labrador G, Viera-Vera J, Rivera-Vega MY, Startek J, Latorre-Esteves M, Román-Colón A, Rivera-Torres R, Navarro-Millán IY, GómezSánchez E, Caro-González HY, Valencia-Rivera P. 2005. Reconstructing the population history of Puerto Rico by means of mtDNA phylogeographic analysis. Am J Phys Anthropol 128:131-155.

Mason P. 2000. Jamaica: a guide to the people, politics and culture. Brooklyn, NY: Interlink Books. 
McLean DC, Spruill I, Argyropoulos G, Page GP, Shriver MD, Garvey WT. 2005. Mitochondrial DNA (mtDNA) haplotypes reveal maternal population genetic affinities of Sea Island Gullah-Speaking African Americans. Am J Phys Anthropol 127:427-438.

McLean DC, Spruill I, Gevao S, Morrison EYSA, Bernard OS, Argyropoulos G, Garvey WT. 2003. Three novel mtDNA restriction site polymorphisms allow exploration of population affinities of African Americans. Hum Biol 75:147-161.

Mendizabal I, Sandoval K, Berniell-Lee G, Calafell F, Salas A, Martínez-Fuentes A, Comas D. 2008. Genetic origin, admixture, and asymmetry in maternal and paternal human lineages in Cuba. BMC Genet 8:213.

Mertens G, Mommers N, Cardoen E, De Bruyn I, Jehaes E, Rand S, Van Brussel K, Jacobs W. 2006. Flemish population genetic analysis using 15 STRs of the Identifiler ${ }^{\circledR}$ kit. Int Congr Ser 1288:328-330.

Miljkovic-Gacic I, Ferrell RE, Patrick AL, Kammerer CM, Bunker CH. 2005. Estimates of African, European and Native American Ancestry in Afro-Caribbean men on the island of Tobago. Hum Hered 60:129-133.

Mordecai M, Mordecai P. 2001. Culture and customs of Jamaica. Westport, CT: Greenwood Press.

Muro T, Fujihara J, Imamura S, Nakamura H, Yasuda T, Takeshita H. 2008. Allele frequencies for 15 STR loci in Ovambo population using AmpFlSTR ${ }^{\circledR}$ Identifiler kit. Leg Med 10:157-159.

Nagel RL. 1984. The origin of the hemoglobin S gene: clinical, genetic and anthropological consequences. Einstein Q J Biol Med 2:53-62.

Okediji MB. 2003. The shattered gourd: Yoruba forms in twentieth-century American art. Seattle, WA: The University of Washington Press.

Ota T. 1993. DISPAN: genetic distance and phylogenetic analysis. University Park, PA: Institute of Molecular Evolutionary Genetics, Pennsylvania State University.

Ott T. 1973. The Haitian Revolution 1789-1804. Knoxville, TN: The University of Tennessee Press.

Pamphile LD. 2001. Haitians and African Americans. Gainseville, FL: University Press of Florida.

Pante-de-Sousa G, Mousinho-Ribeiro RC, Santos EJM, ZagoMA, Guerreiro JF. 1998. Origin of the Hemoglobin S gene in a northern Brazilian population: the combined effects of slave trade and internal migrations. Genet Mol Biol 21:427-430. 
Parra EJ, Marcini A, Akey J, Martinson J, Batzer MA, Cooper R, Forrester T, Allison DB, Deka R, Ferrell RE, Shriver MD. 1998. Estimating African American admixture proportions by use of population-specific alleles. Am J Hum Genet 63:1839-1851.

Pepin J. 2005. From the Old World to the New World: an ecologic study of population susceptibility to HIV infection. Trop Med Int Health 10:627-639.

Pereira JV, Serra A, Anjos MJ, Vide MC, Corte-Real F, Vieira DN. 2005. Allele frequencies for fifteen STR loci in Guinea-Bissau population. J Forensic Sci 50:243-244.

Pereira L, Macaulay V, Torroni A, Scozzari R, Prata MJ, Amorim A. 2001. Prehistoric and historic traces in the mtDNA of Mozambique: insights into the Bantu expansions and the slave trade. Am J Hum Genet 65:439-458.

Pritchard JK, Stephens M, Donnelly P. 2000. Inference of population structure using multilocus genotype data. Genetics 155:945-959.

Raymond M, Rousset F. 1995. GENEPOP (version 1.2): a population genetics software for exact test and ecumenicism. J Hered 86:248-249.

Rebala K, Wysocka J, Kapińska E, Cybulska L, Mikulich AI, Tsybovsky IS, Szczerkowska Z. 2007. Belarusian population genetic database for 15 autosomal STR loci. Forensic Sci Int 173:235-237.

Regueiro M, Carril JC, Pontes ML, Pinheiro MF, Luis JR, Caeiro B. 2004. Allele distribution of 15 PCR-based loci in the Rwanda Tutsi population by multiplex amplification and capillary electrophoresis. Forensic Sci Int 143:61-63.

Regueiro M, Mirabal S, Lacau H, Caeiro JL, Garcia-Bertrand RL, Herrera RJ. 2008. Austronesian genetic signature in East African Madagascar and Polynesia. J Hum Genet 53:106-120.

Roberts DF, Hiorns RW. 1965. Methods of analysis of the genetic composition of a hybrid population. Hum Biol 37:38-43.

Roberts GW. 1954. Immigration of Africans into the British Caribbean. Population Studies 7:235-262.

Roberts K. 2004. The influential Yoruba past in Haiti. In: Falolola T, Childs MD, editors. The Yoruba diaspora in the Atlantic world. Bloomington, IN: Indiana University Press. p 177-182.

Rodríguez A, Arrieta G, Sanóu I, Vargas MC, García O, Yurrebaso I, Pérez JA, Villalta M, Espinoza M. 2007. Population genetic data for 18 STR loci in Costa Rica. Forensic Sci Int 168:85-88. 
Rogoziński J. 1999. A brief history of the Caribbean. New York, NY: Plume.

Salas A, Richards M, De la Fe T, Lareu M, Sobrino B, Sánchez-Diz P, Macaulay V, Carracedo Á. 2002. The making of the African mtDNA landscape. Am J Hum Genet 71:1082-1111.

Saunders G. 2003. Cultural Perspectives. In: Bahamas Department of Archives Bahamas Heritage Festival. Nassau, Bahamas: Government Printing Department. p 1-13.

Schneider S, Kueffer JM, Roessli D, Excoffler L. 2000. Arlequin v. 2.000: a software for population genetics data analysis. Geneva: Genetics and Biometry Laboratory, University of Geneva.

Schuler M. 1979. Myalism and the African religious tradition in Jamaica. In: Knight F, Crahan M, editors. Africa and the Caribbean. Baltimore, MA: The Johns Hopkins University Press. p 65-79.

Seah LH, Jeevan NH, Othman MI, Jaya P, Ooi YS, Wong PC, Kee SS. 2003. STR data for the AmpFISTR Identifiler loci in three ethnic groups (Malay, Chinese, Indian) of the Malaysian population. Forensic Sci Int 138:134-137.

Shepard EM, Herrera RJ. 2006. Genetic encapsulation among Near Eastern populations. J Hum Genet 51:467-476.

Simms TM, Garcia C, Mirabal S, McCartney Q, Herrera RJ. 2008. The genetic legacy of the Transatlantic Slave Trade in the island of New Providence. Forensic Sci Int: Genet 2:310-317.

SPSS for Windows, Rel. 11.0.1. 2006. Chicago: SPSS Inc.

Thomas H. 1998. The slave trade: the history of the Atlantic slave trade 1440-1870. London: Macmillan Publishers Ltd.

Thomas-Hope E. 2003. Irregular Migration and Asylum Seekers in the Caribbean. United Nations University, WIDER. Discussion Paper No. 2003/48.

Treco RN. 2002. The Haitian diaspora in the Bahamas. Miami, FL: Florida International University.

Tselos SE. 1996. Threads of reflection: costumes of Haitian Rara. Afr Arts Special Issue Arts of Vodou 29:58-102. 
Vergara C, Caraballo L, Mercado D, Jimenez S, Rojas W, Rafaels N, Hand T, Campbell M, Tsai YJ, Gao L, Duque C, Lopez S, Bedoya G, Ruiz-Linares A, Barnes KC. 2009. African ancestry is associated with risk of asthma and high total serum $\operatorname{IgE}$ in a population from the Caribbean Coast of Colombia. Hum Genet 125:565-579.

Wainscoat JS, Bell JI, Thein SL, Higgs DR, Serjeant GR, Peto TEA, Weatherall DJ. 1983. Multiple origins of the sickle mutation: evidence from $\beta^{S}$ gene cluster polymorphism. Mol Biol Med 1:191-197.

Wang J. 2003. Maximum-likelihood estimation of admixture proportions from genetic data. Genetics 164:747-765.

Wang Z, Yu R, Wang F, Li X, Jin T. 2005. Genetic polymorphisms of 15 STR loci in Han population from Shaanxi (NW China). Forensic Sci Int 147:89-91.

Watts D. 1987. The West Indies patterns of development, cultural and environmental change since 1492. New York, NY: Columbia University Press.

Zúñiga J, Ilzarbe M, Acunha-Alonzo V, Rosetti F, Herbet Z, Romero V, Almeciga I, Clavijo O, Stern JNH, Granados J, Fridkis-Hareli M, Morrison P, Azocar J, Yunis EJ. 2006. Allele frequencies for 15 autosomal STR loci and admixture estimates in Puerto Rican Americans. Forensic Sci Int 164:266-270. 


\section{Appendix IIII}

Fig. 1A: Multidimensional Scaling plot $($ Stress $=0.10202)$ of all populations at 15 loci

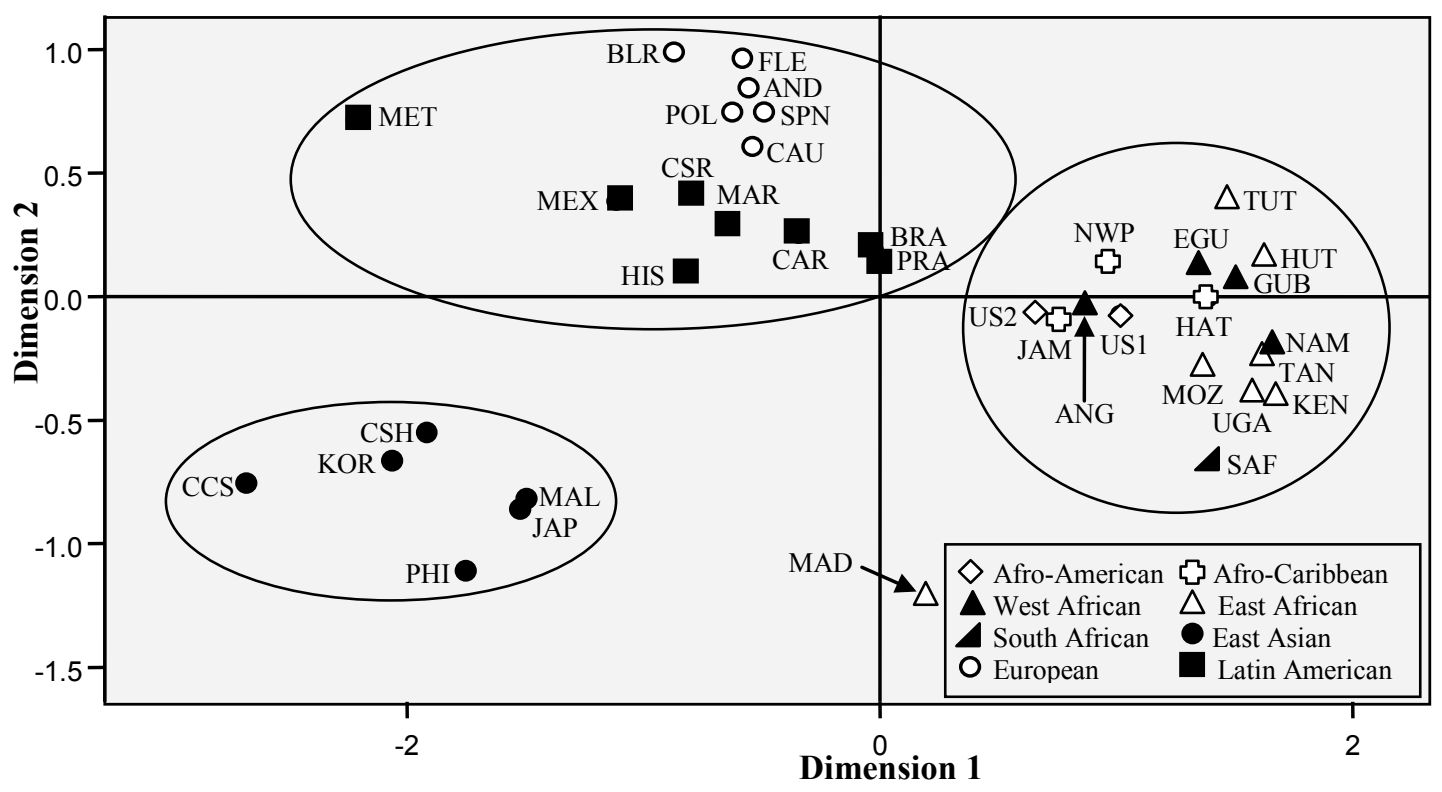

Fig. 1B: Multidimensional Scaling plot $($ Stress $=0.19149)$ of the African derived collections

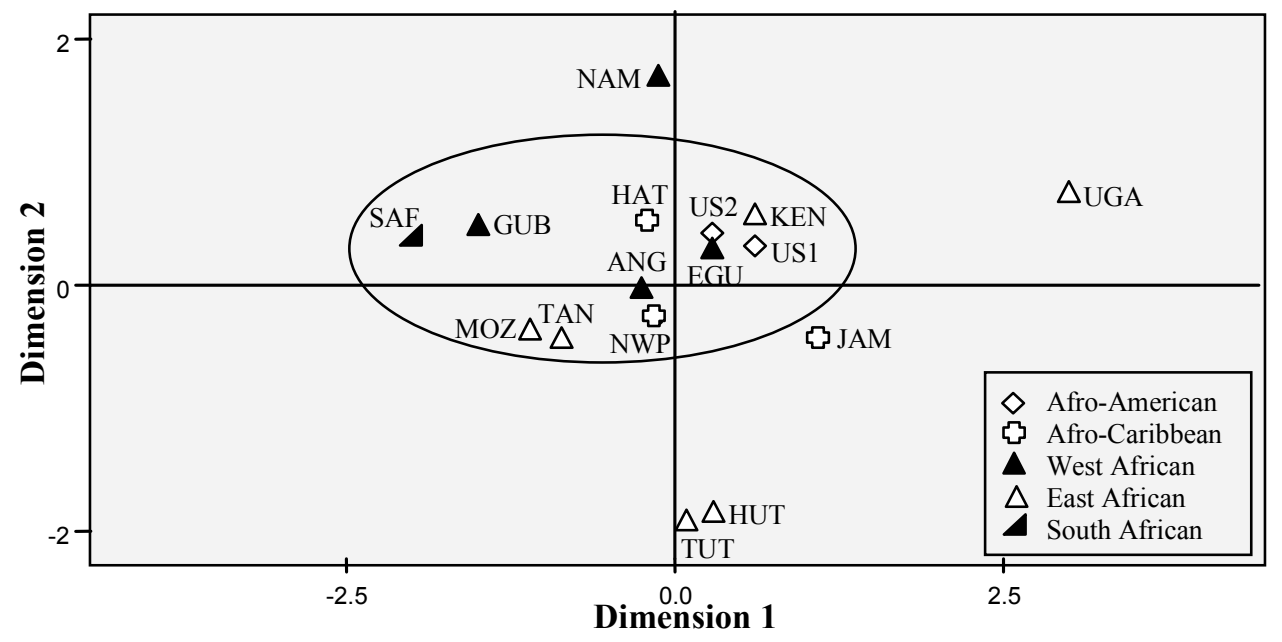




\begin{tabular}{|c|c|c|c|c|}
\hline Population & Abbreviation & $\mathbf{n}$ & $\operatorname{Avg~} H_{e}$ & Reference \\
\hline \multicolumn{5}{|l|}{ AFRO-CARIBBEAN $^{\mathrm{a}}$} \\
\hline Haiti & HAT & 111 & 0.80300 & present study \\
\hline Jamaica & JAM & 119 & 0.77647 & present study \\
\hline New Providence (Bahamas) & NWP & 221 & 0.78312 & Simms et al., 2008 \\
\hline \multicolumn{5}{|l|}{ AFRO-AMERICAN ${ }^{\mathrm{a}}$} \\
\hline US African American 1 (Minnesota) & US1 & 157 & 0.80380 & Gross and Budowle, 2006 \\
\hline US African American 2 (General) & US2 & 258 & 0.79573 & Butler et al., 2003 \\
\hline \multicolumn{5}{|l|}{ WEST AFRICAN } \\
\hline Cabinda (Angola) & ANG & 110 & 0.79698 & Beleza et al., 2004 \\
\hline Guinea-Bissau & GUB & 92 & 0.80249 & Pereira et al., 2005 \\
\hline Equatorial Guinea & EGU & 134 & 0.89193 & Alves et al., 2005 \\
\hline Namibia & NAM & 195 & 0.78293 & Muro et al., 2008 \\
\hline \multicolumn{5}{|l|}{ EAST AFRICAN } \\
\hline Hutu (Rwanda) & HUT & 95 & 0.81193 & Shepard and Herrera, 2006 \\
\hline Kenya & KEN & 65 & 0.81436 & Shepard and Herrera, 2006 \\
\hline Madagascar & MAD & 67 & 0.80350 & Regueiro et al., 2008 \\
\hline Maputo (Mozambique) & MOZ & 144 & 0.79580 & Alves et al., 2004 \\
\hline Tanzania & TAN & 272 & 0.81062 & Forward et al., 2008 \\
\hline Tutsi (Rwanda) & TUT & 126 & 0.77818 & Regueiro et al., 2004 \\
\hline Uganda & UGA & 90 & - & Gusmão et al., 2006 \\
\hline \multicolumn{5}{|l|}{ SOUTH AFRICAN } \\
\hline Cape Town (South Africa) & SAF & 98 & - & Kido et al., 2007 \\
\hline \multicolumn{5}{|l|}{ EAST ASIAN } \\
\hline Chao Shan (South China) & $\mathrm{CCS}$ & 144 & 0.77793 & Hu et al., 2005 \\
\hline Japan & JAP & 526 & 0.77127 & Hashiyada et al., 2003 \\
\hline Korea & KOR & 231 & 0.77087 & Kim et al., 2003 \\
\hline Malaysia & MAL & 210 & 0.78127 & Seah et al., 2003 \\
\hline Philippines & PHI & 106 & 0.77793 & De Ungria et al., 2005 \\
\hline Shaanxi Han (NW China) & $\mathrm{CSH}$ & 203 & 0.78232 & Wang et al., 2005 \\
\hline \multicolumn{5}{|l|}{ LATIN AMERICAN } \\
\hline Caracas (Central Venezuela) & CAR & 255 & 0.79267 & Chiurillo et al., 2003 \\
\hline Central Mexico & MEX & 211 & 0.82827 & Hernández-Gutiérrez et al., 2005 \\
\hline Costa Rica & CSR & 500 & 0.85353 & Rodríguez et al., 2007 \\
\hline Dominican Republic ${ }^{\mathbf{b}}$ & DMR & 2565 & 0.89677 & Díaz et al., 2008 \\
\hline Hispanic (US -Minnesota) & HIS & 151 & 0.80120 & Gross and Budowle, 2006 \\
\hline Maracaibo (Venezuela) & MAR & 203 & 0.77896 & Bernal et al., 2006 \\
\hline Metztitlán (Mexico) & MET & 180 & 0.75067 & Gorostiza et al., 2007 \\
\hline Puerto Rico American (Massachusetts) & PRA & 205 & 0.79113 & Zúñiga et al., 2006 \\
\hline Rio de Janeiro (Brazil) & BRA & 300 & 0.85533 & de Souza Góes et al., 2004 \\
\hline \multicolumn{5}{|l|}{ EUROPEAN } \\
\hline Andalusia (South Spain) & AND & 114 & 0.77600 & Coudray et al., 2007 \\
\hline Belaruse & BLR & 176 & 0.77160 & Rebala et al., 2007 \\
\hline Caucasian (US - General) & $\mathrm{CAU}$ & 302 & 0.78173 & Butler et al., 2003 \\
\hline Flemish (North Belgium) & FLE & 231 & 0.79533 & Mertens et al., 2006 \\
\hline North and Central Poland & POL & 412 & 0.79447 & Czarny et al, 2005 \\
\hline Spain & SPN & 342 & 0.76666 & Camacho et al., 2007 \\
\hline
\end{tabular}

${ }^{a}$ These groups are combined in some analyses as New World Africans. For specific analyses see Materials \& Methods.

${ }^{b}$ The Average Heterozygosity $\left(\mathrm{Avg} \mathrm{H}_{\mathrm{e}}\right.$ ) for this group was calculated at the level of 13 loci.

Table 1: Populations Analyzed 


\begin{tabular}{|c|c|c|c|c|c|c|c|c|c|c|c|c|c|}
\hline allele & $\mathbf{5}$ & $\mathbf{6}$ & $\mathbf{7}$ & $\mathbf{8}$ & $\mathbf{9}$ & $\mathbf{9 . 3}$ & $\mathbf{1 0}$ & $\mathbf{1 0 . 1}$ & $\mathbf{1 0 . 2}$ & $\mathbf{1 1}$ & $\mathbf{1 2}$ & $\mathbf{1 2 . 2}$ & $\mathbf{1 3}$ \\
\hline D8S1179 & & & & 0.0045 & & & 0.0090 & & & 0.0495 & 0.0586 & & 0.1847 \\
\hline D21S11 & & & & & & & & & & & & & \\
\hline D7S820 & & & 0.0045 & 0.2297 & 0.1532 & & 0.3018 & 0.0045 & & 0.1667 & 0.1036 & & 0.0315 \\
\hline CSF1PO & & & 0.0360 & 0.0676 & 0.0225 & & 0.2613 & & & 0.2523 & 0.2883 & & 0.0586 \\
\hline D3S1358 & & & & & & & & & & & & & \\
\hline TH01 & 0.0090 & 0.1036 & 0.4775 & 0.2207 & 0.1486 & 0.0315 & 0.0090 & & & & & & \\
\hline D13S317 & & & & 0.0045 & 0.0090 & & 0.0270 & & & 0.2928 & 0.4730 & & 0.1306 \\
\hline D16S539 & 0.0090 & 0.0045 & & 0.0495 & 0.1937 & & 0.0856 & & & 0.2793 & 0.2027 & & 0.1351 \\
\hline D2S1338 & & & & & & & & & & & & & \\
\hline D19S433 & & & & & & & 0.0180 & & & 0.0450 & 0.1081 & 0.0495 & 0.2838 \\
\hline vWA & & & & & & & & & & 0.0045 & 0.0045 & & 0.0045 \\
\hline TPOX & & 0.0946 & 0.0135 & 0.2928 & 0.2117 & & 0.0811 & & & 0.2748 & 0.0270 & & 0.0045 \\
\hline D18S51 & & & & & 0.0045 & & 0.0045 & & 0.0045 & & 0.0586 & & 0.0405 \\
\hline D5S818 & & & & 0.0631 & 0.0180 & & 0.0721 & & & 0.1937 & 0.3784 & & 0.2387 \\
\hline FGA & & & & & & & & & & & & & \\
\hline
\end{tabular}

\begin{tabular}{|c|c|c|c|c|c|c|c|c|c|c|c|c|c|}
\hline allele & $\mathbf{1 3 . 2}$ & $\mathbf{1 4}$ & $\mathbf{1 4 . 2}$ & $\mathbf{1 5}$ & $\mathbf{1 5 . 2}$ & $\mathbf{1 6}$ & $\mathbf{1 6 . 1}$ & $\mathbf{1 6 . 2}$ & $\mathbf{1 7}$ & $\mathbf{1 7 . 2}$ & $\mathbf{1 8}$ & $\mathbf{1 8 . 2}$ & $\mathbf{1 9}$ \\
\hline D8S1179 & & 0.3874 & & 0.2432 & & 0.0541 & & & 0.0090 & & & & \\
\hline D21S11 & & & & & & & & & & & & & \\
\hline D7S820 & & 0.0045 & & & & & & & & & & & \\
\hline CSF1PO & & 0.0090 & & 0.0045 & & & & & & & & & \\
\hline D3S1358 & & 0.1261 & & 0.3063 & & 0.3108 & & & 0.2117 & & 0.0360 & & 0.0090 \\
\hline TH01 & & & & & & & & & & & & & \\
\hline D13S317 & & 0.0631 & & & & & & & & & & & \\
\hline D16S539 & & 0.0360 & & 0.0045 & & & & & & & & & \\
\hline D2S1338 & & & & & & 0.0676 & & & 0.1081 & & 0.0405 & & 0.1712 \\
\hline D19S433 & 0.0495 & 0.1937 & 0.0676 & 0.0856 & 0.0586 & 0.0135 & & 0.0180 & 0.0045 & 0.0045 & & & \\
\hline vWA & & 0.0991 & & 0.1937 & & 0.3108 & & & 0.1757 & & 0.1261 & & 0.0721 \\
\hline TPOX & & & & & & & & & & & & & \\
\hline D18S51 & 0.0045 & 0.0586 & 0.0090 & 0.1261 & 0.0045 & 0.2162 & & & 0.1532 & & 0.1036 & & 0.0991 \\
\hline D5S818 & & 0.0270 & & & & 0.0045 & & & 0.0045 & & & & \\
\hline FGA & & & & & & & 0.0045 & & & & 0.0045 & 0.0045 & 0.0450 \\
\hline
\end{tabular}

Table 2: Haiti allelic frequencies $(n=111)$ 


\begin{tabular}{|c|c|c|c|c|c|c|c|c|c|c|c|c|c|}
\hline allele & $\mathbf{2 0}$ & $\mathbf{2 0 . 2}$ & $\mathbf{2 1}$ & $\mathbf{2 1 . 2}$ & $\mathbf{2 2}$ & $\mathbf{2 3}$ & $\mathbf{2 4}$ & $\mathbf{2 4 . 3}$ & $\mathbf{2 5}$ & $\mathbf{2 6}$ & $\mathbf{2 7}$ & $\mathbf{2 8}$ & $\mathbf{2 9}$ \\
\hline D8S1179 & & & & & & & & & & & & & \\
\hline D21S11 & & & & & & & & & & 0.0045 & 0.0315 & 0.3018 & 0.1532 \\
\hline D7S820 & & & & & & & & & & & & & \\
\hline CSF1P0 & & & & & & & & & & & & & \\
\hline D3S1358 & & & & & & & & & & & & & \\
\hline TH01 & & & & & & & & & & & & & \\
\hline D13S317 & & & & & & & & & & & & & \\
\hline D16S539 & & & & & & & & & & & & & \\
\hline D2S1338 & 0.0676 & & 0.1396 & & 0.1396 & 0.1261 & 0.0676 & & 0.0631 & 0.0090 & & & \\
\hline D19S433 & & & & & & & & & & & & & \\
\hline vWA & & & 0.0045 & & 0.0045 & & & & & & & & \\
\hline TPOX & & & & & & & & & & & & & \\
\hline D18S51 & 0.0856 & & 0.0180 & 0.0045 & 0.0045 & & & & & & & & \\
\hline D5S818 & & & & & & & & & & & & & \\
\hline FGA & 0.0586 & 0.0045 & 0.0991 & & 0.1847 & 0.1802 & 0.1667 & 0.0045 & 0.1486 & 0.0495 & 0.0315 & & \\
\hline
\end{tabular}

\begin{tabular}{|c|c|c|c|c|c|c|c|c|c|c|c|c|}
\hline allele & 30 & 30.2 & 31 & 31.2 & 32 & 32.2 & 33 & 33.2 & 34 & 34.2 & 35 & 36 \\
\hline \multicolumn{13}{|l|}{ D8S1179 } \\
\hline D21S11 & 0.2072 & 0.0180 & 0.0721 & 0.0631 & 0.0225 & 0.0495 & 0.0045 & 0.0090 & 0.0135 & 0.0045 & 0.0405 & 0.0045 \\
\hline \multicolumn{13}{|l|}{ D7S820 } \\
\hline \multicolumn{13}{|l|}{ CSF1PO } \\
\hline \multicolumn{13}{|l|}{ D3S1358 } \\
\hline \multicolumn{13}{|l|}{ TH01 } \\
\hline \multicolumn{13}{|l|}{ D13S317 } \\
\hline \multicolumn{13}{|l|}{ D16S539 } \\
\hline \multicolumn{13}{|l|}{ D2S1338 } \\
\hline \multicolumn{13}{|l|}{ D19S433 } \\
\hline \multicolumn{13}{|l|}{ vWA } \\
\hline \multicolumn{13}{|l|}{ TPOX } \\
\hline \multicolumn{13}{|l|}{ D18S51 } \\
\hline \multicolumn{13}{|l|}{ D5S818 } \\
\hline FGA & & 0.0045 & & 00090 & & & & & & & & \\
\hline
\end{tabular}

Table 2: Haiti allelic frequencies $(n=111)$ (Cont'd) 


\begin{tabular}{|c|c|c|c|c|c|c|c|c|c|c|c|c|c|}
\hline allele & 6 & 7 & 8 & 9 & 9.3 & 10 & 10.2 & 11 & 11.2 & 12 & 12.2 & 13 & 13.2 \\
\hline D8S1179 & & & & 0.0126 & & 0.0378 & & 0.0714 & & 0.1134 & & 0.1513 & \\
\hline \multicolumn{14}{|l|}{ D21S11 } \\
\hline D7S820 & 0.0084 & 0.0084 & 0.1639 & 0.0798 & & 0.3992 & & 0.2101 & & 0.1176 & & 0.0084 & \\
\hline CSF1PO & & 0.0546 & 0.0672 & 0.0420 & & 0.2941 & & 0.2017 & & 0.2647 & & 0.0630 & \\
\hline D3S1358 & & & & & & & & & & 0.0042 & & & \\
\hline ТH01 & 0.1429 & 0.3655 & 0.2311 & 0.1555 & 0.0840 & 0.0210 & & & & & & & \\
\hline D13S317 & & & 0.0336 & 0.0294 & & 0.0336 & & 0.2731 & & 0.4244 & & 0.1765 & \\
\hline D16S539 & & & 0.0168 & 0.2521 & & 0.1134 & & 0.2983 & & 0.2101 & & 0.0924 & \\
\hline \multicolumn{14}{|l|}{ D2S1338 } \\
\hline D19S433 & & & & 0.0042 & & 0.0084 & & 0.0546 & 0.0084 & 0.1218 & 0.0546 & 0.2563 & 0.0462 \\
\hline vWA & & & & & & & & 0.0084 & & & & 0.0084 & \\
\hline TPOX & 0.0336 & 0.0210 & 0.4286 & 0.1807 & & 0.0714 & & 0.2437 & & 0.0210 & & & \\
\hline D18S51 & & & & & & & 0.0042 & 0.0084 & & 0.0714 & & 0.0630 & 0.0042 \\
\hline D5S818 & & & 0.0588 & 0.0210 & & 0.0420 & & 0.2689 & & 0.3445 & & 0.2479 & \\
\hline FGA & & & & & & & & & & & & & \\
\hline
\end{tabular}

\begin{tabular}{|c|c|c|c|c|c|c|c|c|c|c|c|c|c|}
\hline allele & 14 & 14.2 & 15 & 15.2 & 16 & 16.1 & 16.2 & 17 & 17.2 & 18 & 18.2 & 19 & 19.2 \\
\hline D8S1179 & 0.2857 & & 0.2227 & & 0.0882 & & & 0.0168 & & & & & \\
\hline \multicolumn{14}{|l|}{ D21S11 } \\
\hline D7S820 & 0.0042 & & & & & & & & & & & & \\
\hline CSF1PO & 0.0126 & & & & & & & & & & & & \\
\hline D3S1358 & 0.0630 & & 0.2983 & 0.0042 & 0.3109 & & & 0.2521 & & 0.0588 & & 0.0084 & \\
\hline \multicolumn{14}{|l|}{ TH01 } \\
\hline D13S317 & 0.0294 & & & & & & & & & & & & \\
\hline D16S539 & 0.0168 & & & & & & & & & & & & \\
\hline D2S1338 & & & & & 0.0378 & & & 0.0966 & & 0.0630 & & 0.2017 & \\
\hline D19S433 & 0.2185 & 0.0588 & 0.0798 & 0.0252 & 0.0168 & & 0.0378 & 0.0042 & 0.0042 & & & & \\
\hline vWA & 0.0630 & & 0.2437 & & 0.2479 & & & 0.1681 & & 0.1345 & & 0.0882 & \\
\hline \multicolumn{14}{|l|}{ TPOX } \\
\hline D18S51 & 0.0798 & 0.0084 & 0.1429 & & 0.1975 & & & 0.1513 & & 0.1092 & & 0.1050 & \\
\hline D5S818 & 0.0084 & & 0.0042 & & 0.0042 & & & & & & & & \\
\hline FGA & & & & & & 0.0042 & & & & & 0.0252 & 0.0588 & 0.0042 \\
\hline
\end{tabular}

\begin{tabular}{|c|c|c|c|c|c|c|c|c|c|c|c|c|c|}
\hline allele & 20 & 20.2 & 21 & 21.2 & 22 & 23 & 24 & 25 & 26 & 27 & 28 & 29 & 30 \\
\hline \multicolumn{14}{|l|}{ D8S1179 } \\
\hline D21S11 & & & & & & & & & 0.0042 & 0.0336 & 0.2521 & 0.2101 & 0.1849 \\
\hline \multicolumn{14}{|l|}{ D7S820 } \\
\hline \multicolumn{14}{|l|}{ CSF1PO } \\
\hline \multicolumn{14}{|l|}{ D3S1358 } \\
\hline \multicolumn{14}{|l|}{ TH01 } \\
\hline \multicolumn{14}{|l|}{ D13S317 } \\
\hline \multicolumn{14}{|l|}{ D16S539 } \\
\hline D2S1338 & 0.1176 & & 0.1218 & & 0.1092 & 0.0966 & 0.0840 & 0.0588 & 0.0084 & 0.0042 & & & \\
\hline \multicolumn{14}{|l|}{ D19S433 } \\
\hline vWA & 0.0252 & & 0.0042 & & 0.0084 & & & & & & & & \\
\hline \multicolumn{14}{|l|}{ TPOX } \\
\hline D18S51 & 0.0336 & & 0.0084 & & 0.0084 & 0.0042 & & & & & & & \\
\hline \multicolumn{14}{|l|}{ D5S818 } \\
\hline FGA & 0.0336 & 0.0042 & 0.1050 & 0.0084 & 0.1597 & 0.1891 & 0.2017 & 0.1008 & 0.0420 & 0.0336 & 0.0168 & 0.0042 & 0.0042 \\
\hline
\end{tabular}

Table 3: Jamaica allelic frequencies $(n=119)$ 


\begin{tabular}{|c|c|c|c|c|c|c|c|c|c|c|c|}
\hline allele & $\mathbf{3 0 . 2}$ & $\mathbf{3 1}$ & $\mathbf{3 1 . 2}$ & $\mathbf{3 2}$ & $\mathbf{3 2 . 2}$ & $\mathbf{3 3}$ & $\mathbf{3 3 . 2}$ & $\mathbf{3 4 . 2}$ & $\mathbf{3 5}$ & $\mathbf{3 6}$ & $\mathbf{4 5 . 2}$ \\
\hline D8S1179 & & & & & & & & & & & \\
\hline D21S11 & 0.0042 & 0.0630 & 0.0546 & 0.0294 & 0.0630 & 0.0084 & 0.0378 & 0.0084 & 0.0336 & 0.0126 & \\
\hline D7S820 & & & & & & & & & & & \\
\hline CSF1PO & & & & & & & & & & & \\
\hline D3S1358 & & & & & & & & & & & \\
\hline TH01 & & & & & & & & & & \\
\hline D13S317 & & & & & & & & & & \\
\hline D16S539 & & & & & & & & & & \\
\hline D2S1338 & & & & & & & & & & & \\
\hline D19S433 & & & & & & & & & & \\
\hline vWA & & & & & & & & & & & \\
\hline TPOX & & & & & & & & & & \\
\hline D18S51 & & & & & & & & & & & \\
\hline D5S818 & & & & & & & & & & \\
\hline FGA & & & & & & & & & & & 0.0042 \\
\hline
\end{tabular}

Table 3: Jamaica allelic frequencies $(n=119)($ Cont'd)

\begin{tabular}{|c|c|c|c|c|c|c|c|c|}
\hline \multirow[b]{2}{*}{ Locus } & \multicolumn{4}{|c|}{ Haiti } & \multicolumn{4}{|c|}{ Jamaica } \\
\hline & $\mathbf{H}_{\mathbf{0}}$ & $\mathbf{H}_{\mathrm{e}}$ & HWE & GDI & $\mathbf{H}_{\mathbf{o}}$ & $\mathbf{H}_{\mathrm{e}}$ & HWE & GDI \\
\hline D8S1179 & 0.8198 & 0.7511 & 0.3208 & 0.7511 & 0.8740 & 0.8217 & 0.5772 & 0.8217 \\
\hline D21S11 & 0.8559 & 0.8308 & 0.3520 & 0.8308 & 0.7983 & 0.8459 & 0.3424 & 0.8459 \\
\hline D7S820 & 0.7387 & 0.7967 & 0.3609 & 0.7967 & 0.7143 & 0.7524 & 0.9637 & 0.7524 \\
\hline CSF1PO & 0.8378 & 0.7786 & 0.4647 & 0.7786 & 0.7899 & 0.7927 & 0.9435 & 0.7927 \\
\hline D3S1358 & 0.7207 & 0.7509 & 0.6197 & 0.7508 & 0.7227 & 0.7464 & 0.7683 & 0.7464 \\
\hline TH01 & 0.6757 & 0.6924 & 0.0740 & 0.6924 & 0.6555 & 0.7641 & 0.1598 & 0.7641 \\
\hline D13S317 & 0.6306 & 0.6717 & 0.7018 & 0.6717 & 0.6807 & 0.7132 & 0.1949 & 0.7132 \\
\hline D16S539 & 0.8198 & 0.8176 & 0.9226 & 0.8176 & 0.7563 & 0.7846 & 0.0178 & 0.7846 \\
\hline D2S1338 & 0.8919 & 0.8887 & 0.4855 & 0.8887 & 0.8235 & 0.8877 & 0.0040 & 0.8877 \\
\hline D19S433 & 0.8829 & 0.8510 & 0.2813 & 0.8510 & 0.8319 & 0.8548 & 0.3589 & 0.8548 \\
\hline vWA & 0.8469 & 0.8076 & 0.9874 & 0.8076 & 0.8151 & 0.8237 & 0.4727 & 0.8237 \\
\hline TPOX & 0.8108 & 0.7810 & 0.3768 & 0.7810 & 0.6723 & 0.7202 & 0.6787 & 0.7202 \\
\hline D18S51 & 0.8649 & 0.8809 & 0.9021 & 0.8809 & 0.8908 & 0.8815 & 0.6876 & 0.8815 \\
\hline D5S818 & 0.7568 & 0.7555 & 0.8624 & 0.7555 & 0.7311 & 0.7449 & 0.9691 & 0.7449 \\
\hline FGA & 0.8919 & 0.8685 & 0.6072 & 0.8685 & 0.8908 & 0.8720 & 0.4080 & 0.8720 \\
\hline
\end{tabular}

Table 4: Observed $\left(\mathrm{H}_{0}\right)$ and Expected Heterozygosity $\left(\mathrm{H}_{\mathrm{e}}\right)$, Hardy-Weinberg Equilibrium (HWE) $P$-values and Gene Diversity indices (GDI) for the Haitian and Jamaican populations across the 15 Identifiler loci 


\begin{tabular}{|c|c|c|c|c|c|c|c|c|c|c|c|c|}
\hline \multirow[b]{2}{*}{ Locus } & \multicolumn{3}{|c|}{ Afro-Caribbean } & \multicolumn{3}{|c|}{ New World African ${ }^{1}$} & \multicolumn{3}{|c|}{ African w/o Madagascar } & \multicolumn{3}{|c|}{ African with Madagascar } \\
\hline & $\mathbf{G}_{\text {st }}$ & $\mathbf{H}_{\mathrm{s}}$ & $\mathbf{H}_{\mathrm{t}}$ & $\mathbf{G}_{\text {st }}$ & $\mathbf{H}_{\mathrm{s}}$ & $\mathbf{H}_{\mathbf{t}}$ & $\mathbf{G}_{\mathrm{st}}$ & $\mathbf{H}_{\mathrm{s}}$ & $\mathbf{H}_{\mathrm{t}}$ & $\mathbf{G}_{\text {st }}$ & $\mathbf{H}_{\mathrm{s}}$ & $\mathbf{H}_{\mathrm{t}}$ \\
\hline D8S1179 & 0.00476 & 0.78859 & 0.79236 & 0.00493 & 0.79008 & 0.79399 & 0.01146 & 0.77362 & 0.78259 & 0.01253 & 0.77533 & 0.78517 \\
\hline D21S11 & 0.00262 & 0.83717 & 0.83937 & 0.00249 & 0.84052 & 0.84262 & 0.00937 & 0.85160 & 0.85966 & 0.01000 & 0.85301 & 0.86162 \\
\hline D7S820 & 0.00549 & 0.77387 & 0.77814 & 0.00461 & 0.77871 & 0.78231 & 0.00811 & 0.76163 & 0.76786 & 0.00910 & 0.76344 & 0.77045 \\
\hline CSF1PO & 0.00214 & 0.78204 & 0.78372 & 0.00187 & 0.78084 & 0.78230 & 0.01527 & 0.77695 & 0.78899 & 0.01457 & 0.77571 & 0.78718 \\
\hline D3S1358 & 0.00213 & 0.74892 & 0.75052 & 0.00349 & 0.75207 & 0.75470 & 0.01227 & 0.73200 & 0.74109 & 0.01231 & 0.72975 & 0.73884 \\
\hline TH01 & 0.00588 & 0.73376 & 0.73810 & 0.00472 & 0.73893 & 0.74243 & 0.01389 & 0.72753 & 0.73778 & 0.01299 & 0.72930 & 0.73889 \\
\hline D13S317 & 0.00331 & 0.70077 & 0.70309 & 0.00247 & 0.70627 & 0.70802 & 0.00704 & 0.70777 & 0.71278 & 0.01095 & 0.71409 & 0.72199 \\
\hline D16S539 & 0.00363 & 0.79869 & 0.80159 & 0.00348 & 0.79425 & 0.79702 & 0.00870 & 0.78878 & 0.79570 & 0.00967 & 0.78850 & 0.79619 \\
\hline D2S1338 & 0.00216 & 0.88510 & 0.88702 & 0.00247 & 0.88726 & 0.88945 & 0.01061 & 0.88152 & 0.89097 & 0.01043 & 0.88107 & 0.89036 \\
\hline D19S433 & 0.00135 & 0.84174 & 0.84288 & 0.00214 & 0.84083 & 0.84263 & 0.01017 & 0.82757 & 0.83607 & 0.01120 & 0.82926 & 0.83865 \\
\hline vWA & 0.00342 & 0.81135 & 0.81414 & 0.00317 & 0.81307 & 0.81565 & 0.00947 & 0.81916 & 0.82700 & 0.00980 & 0.81737 & 0.82546 \\
\hline TPOX & 0.00655 & 0.75879 & 0.76379 & 0.00491 & 0.76191 & 0.76567 & 0.01585 & 0.76994 & 0.78234 & 0.02124 & 0.76262 & 0.77917 \\
\hline D18S51 & 0.00310 & 0.87691 & 0.87964 & 0.00262 & 0.87786 & 0.88017 & 0.00658 & 0.86494 & 0.87067 & 0.00708 & 0.86545 & 0.87162 \\
\hline D5S818 & 0.00273 & 0.74781 & 0.74986 & 0.00259 & 0.75047 & 0.75242 & 0.00627 & 0.74582 & 0.75053 & 0.01009 & 0.75214 & 0.75980 \\
\hline FGA & 0.00229 & 0.87133 & 0.87333 & 0.00378 & 0.87035 & 0.87366 & 0.00776 & 0.87454 & 0.88138 & 0.00897 & 0.87468 & 0.88260 \\
\hline All Loci & 0.00339 & 0.79712 & 0.79984 & 0.00330 & 0.79889 & 0.80154 & 0.01015 & 0.79356 & 0.80169 & 0.01131 & 0.79411 & 0.80320 \\
\hline
\end{tabular}

${ }^{1}$ The New World African assemblage includes the Afro-Caribbean populations along with the two US Afro-American collections

Table 5: Inter-, intra- and total population genetic variance 


\begin{tabular}{|c|c|c|c|}
\hline \multirow[b]{2}{*}{ Locus } & \multicolumn{3}{|c|}{ East Asian } \\
\hline & $\mathbf{G}_{\text {st }}$ & $\mathbf{H}_{\mathrm{s}}$ & $\mathbf{H}_{\mathrm{t}}$ \\
\hline D8S1179 & 0.00762 & 0.84279 & 0.84926 \\
\hline D21S11 & 0.00871 & 0.81690 & 0.82408 \\
\hline D7S820 & 0.00944 & 0.75886 & 0.76608 \\
\hline CSF1PO & 0.00874 & 0.72283 & 0.72920 \\
\hline D3S1358 & 0.01191 & 0.71936 & 0.72803 \\
\hline ТH01 & 0.01452 & 0.69914 & 0.70944 \\
\hline D13S317 & 0.00608 & 0.80024 & 0.80513 \\
\hline D16S539 & 0.00957 & 0.77487 & 0.78235 \\
\hline D2S1338 & 0.00706 & 0.86761 & 0.87377 \\
\hline D19S433 & 0.01380 & 0.79961 & 0.81079 \\
\hline vWA & 0.00681 & 0.79667 & 0.80213 \\
\hline TPOX & 0.00567 & 0.62619 & 0.62976 \\
\hline D18S51 & 0.00742 & 0.85343 & 0.85981 \\
\hline D5S818 & 0.00761 & 0.78432 & 0.79033 \\
\hline FGA & 0.00468 & 0.85688 & 0.86091 \\
\hline All Loci & 0.00858 & 0.78131 & 0.78807 \\
\hline
\end{tabular}

\begin{tabular}{ccc}
\hline \multicolumn{3}{c}{ Latin American } \\
\hline $\mathbf{G}_{\mathbf{s t}}$ & $\mathbf{H}_{\mathbf{s}}$ & $\mathbf{H}_{\mathbf{t}}$ \\
\hline 0.00533 & 0.79687 & 0.80114 \\
0.00407 & 0.83425 & 0.83765 \\
0.00753 & 0.79102 & 0.79702 \\
0.00330 & 0.71758 & 0.71996 \\
0.01200 & 0.75931 & 0.76853 \\
0.01458 & 0.77066 & 0.78206 \\
0.01072 & 0.80819 & 0.81695 \\
0.00764 & 0.77957 & 0.78557 \\
0.00894 & 0.86449 & 0.87229 \\
0.00778 & 0.82661 & 0.83310 \\
0.00751 & 0.79039 & 0.79637 \\
0.00926 & 0.67459 & 0.68089 \\
0.00356 & 0.87685 & 0.87998 \\
0.01213 & 0.71660 & 0.72540 \\
0.00614 & 0.87367 & 0.87907 \\
0.00796 & 0.79204 & 0.79840 \\
\hline
\end{tabular}

\begin{tabular}{ccc}
\hline \multicolumn{3}{c}{ European } \\
\hline $\mathbf{G}_{\text {st }}$ & $\mathbf{H}_{\mathbf{s}}$ & $\mathbf{H}_{\mathbf{t}}$ \\
\hline 0.00469 & 0.80477 & 0.80856 \\
0.00263 & 0.84413 & 0.84636 \\
0.00428 & 0.80119 & 0.80463 \\
0.00529 & 0.72430 & 0.72815 \\
0.00319 & 0.79479 & 0.79733 \\
0.00847 & 0.77510 & 0.78172 \\
0.00354 & 0.77766 & 0.78042 \\
0.00244 & 0.75587 & 0.75772 \\
0.00414 & 0.86760 & 0.87121 \\
0.00290 & 0.76447 & 0.76670 \\
0.00403 & 0.80725 & 0.81051 \\
0.00481 & 0.61521 & 0.61818 \\
0.00457 & 0.87159 & 0.87559 \\
0.00400 & 0.71021 & 0.71306 \\
0.00358 & 0.85508 & 0.85814 \\
0.00415 & 0.78461 & 0.78789 \\
\hline
\end{tabular}

\begin{tabular}{ccc}
\hline \multicolumn{3}{c}{ All populations } \\
\hline $\mathbf{G}_{\text {st }}$ & $\mathbf{H}_{\mathbf{s}}$ & $\mathbf{H}_{\mathbf{t}}$ \\
\hline 0.02144 & 0.79769 & 0.81517 \\
0.01917 & 0.83997 & 0.85639 \\
0.02157 & 0.77684 & 0.79397 \\
0.01486 & 0.74692 & 0.75819 \\
0.01623 & 0.74802 & 0.76036 \\
0.05882 & 0.74208 & 0.78845 \\
0.03782 & 0.75766 & 0.78744 \\
0.01811 & 0.77984 & 0.79423 \\
0.02051 & 0.87396 & 0.89225 \\
0.02022 & 0.81494 & 0.83175 \\
0.01900 & 0.80596 & 0.82156 \\
0.03776 & 0.69746 & 0.72483 \\
0.01820 & 0.86864 & 0.88474 \\
0.02917 & 0.74265 & 0.76496 \\
0.01136 & 0.86781 & 0.87778 \\
0.02400 & 0.79070 & 0.81014 \\
\hline
\end{tabular}

Table 5: Inter-, intra- and total population genetic variance (Cont'd) 


\begin{tabular}{|c|c|c|c|c|c|c|c|c|c|c|c|c|}
\hline \multirow[b]{2}{*}{ Locus } & \multicolumn{4}{|c|}{ Haiti } & \multicolumn{4}{|c|}{ Jamaica } & \multicolumn{3}{|c|}{ New Providence } & \multirow[b]{2}{*}{$\boldsymbol{P}$} \\
\hline & $\mathbf{H}_{\mathbf{o}}$ & $\mathbf{H}_{\mathbf{e}}$ & $\mathbf{F}_{\text {IS }}$ & $\boldsymbol{P}$ & $\mathbf{H}_{\mathbf{o}}$ & $\mathbf{H}_{\mathbf{e}}$ & $\mathbf{F}_{\text {IS }}$ & $\boldsymbol{P}$ & $\mathbf{H}_{\mathbf{o}}$ & $\mathbf{H}_{\mathbf{e}}$ & $\mathbf{F}_{\text {IS }}$ & \\
\hline D8S1179 & 0.8198 & 0.7511 & -0.0920 & 0.9934 & 0.8740 & 0.8217 & -0.0640 & 0.9383 & 0.8326 & 0.8017 & -0.0390 & 0.8563 \\
\hline D21S11 & 0.8559 & 0.8308 & -0.0300 & 0.1716 & 0.7983 & 0.8459 & 0.0560 & 0.1832 & 0.7783 & 0.8441 & 0.0780 & 0.0019 \\
\hline D7S820 & 0.7387 & 0.7967 & 0.0730 & 0.0270 & 0.7143 & 0.7524 & 0.0510 & 0.3209 & 0.7376 & 0.8287 & 0.0560 & 0.0792 \\
\hline CSF1PO & 0.8378 & 0.7786 & -0.0760 & 0.7320 & 0.7899 & 0.7927 & 0.0040 & 0.5900 & 0.7195 & 0.8057 & 0.0820 & 0.0016 \\
\hline D3S1358 & 0.7207 & 0.7509 & 0.0400 & 0.4083 & 0.7227 & 0.7464 & 0.0320 & 0.5445 & 0.7557 & 0.7577 & 0.0030 & 0.4488 \\
\hline TH01 & 0.6757 & 0.6924 & 0.0240 & 0.0162 & 0.6555 & 0.7641 & 0.1430 & 0.0163 & 0.7557 & 0.7619 & -0.0040 & 0.4157 \\
\hline D13S317 & 0.6306 & 0.6717 & 0.0610 & 0.4027 & 0.6807 & 0.7132 & 0.0460 & 0.4918 & 0.7104 & 0.7767 & 0.0200 & 0.0597 \\
\hline D16S539 & 0.8198 & 0.8176 & -0.0030 & 0.5326 & 0.7563 & 0.7846 & 0.0360 & 0.1389 & 0.7919 & 0.8076 & 0.0130 & 0.0946 \\
\hline D2S1338 & 0.8919 & 0.8887 & -0.0040 & 0.6875 & 0.8235 & 0.8877 & 0.0730 & 0.0147 & 0.8552 & 0.8925 & 0.0380 & 0.0580 \\
\hline D19S433 & 0.8829 & 0.8510 & -0.0380 & 0.8002 & 0.8319 & 0.8548 & 0.0270 & 0.3725 & 0.7873 & 0.8292 & 0.0500 & 0.0491 \\
\hline $\mathbf{v W A}$ & 0.8469 & 0.8076 & -0.0490 & 0.9187 & 0.8151 & 0.8237 & 0.0100 & 0.3964 & 0.7964 & 0.8117 & 0.0190 & 0.5672 \\
\hline TPOX & 0.8108 & 0.7810 & -0.0380 & 0.7439 & 0.6723 & 0.7202 & 0.0670 & 0.3706 & 0.6833 & 0.7835 & 0.1280 & 0.0150 \\
\hline D18S51 & 0.8649 & 0.8809 & 0.0180 & 0.2617 & 0.8908 & 0.8815 & -0.0110 & 0.6247 & 0.8959 & 0.8815 & -0.0200 & 0.7320 \\
\hline D5S818 & 0.7568 & 0.7555 & -0.0020 & 0.2589 & 0.7311 & 0.7449 & 0.0190 & 0.5483 & 0.7330 & 0.7513 & 0.0240 & 0.0949 \\
\hline FGA & 0.8919 & 0.8685 & -0.0270 & 0.8145 & 0.8908 & 0.8720 & -0.0220 & 0.9037 & 0.9140 & 0.8856 & -0.0350 & 0.0000 \\
\hline
\end{tabular}

$P$ values $<0.05$ are in bold

Table 6: Heterozygote deficiencies for the Afro-Caribbean populations 


\begin{tabular}{lc} 
Locus & WA/EA \\
\hline D8S1179 & 0.107 \\
D21S11 & 0.095 \\
D7S820 & 0.063 \\
CSF1P0 & 0.117 \\
D3S1358 & 0.045 \\
TH01 & 0.104 \\
D13S317 & 0.080 \\
D16S539 & 0.110 \\
D2S1338 & 0.063 \\
D19S433 & 0.078 \\
vWA & 0.063 \\
TPOX & 0.105 \\
D18S51 & 0.086 \\
D5S818 & 0.097 \\
FGA & 0.105 \\
\hline \hline Average & 0.088 \\
& \\
WA: West African \\
EA: East African
\end{tabular}

Table 7: Delta (ס) values for the grouped East vs. West African populations 


\begin{tabular}{|c|c|c|c|c|c|c|c|c|c|c|c|}
\hline & US1 & US2 & ANG & SAF & EGU & GUB & HAT & HUT & JAM & KEN & MAD \\
\hline US1 & & 231.3660 & 272.1790 & 396.6248 & 231.1540 & 284.6625 & 232.8402 & 290.1581 & 186.0889 & 202.7056 & 345.3930 \\
\hline US2 & 0.0506 & & 292.7030 & 399.4874 & 244.9366 & 282.1639 & 227.0359 & 342.1582 & 216.0961 & 217.6445 & 382.9854 \\
\hline ANG & 0.0001 & 0.0000 & & 310.2818 & 221.7053 & 282.4472 & 239.2100 & 260.0201 & 262.8510 & 198.6299 & 371.3746 \\
\hline SAF & 0.0000 & 0.0000 & 0.0000 & & 333.2830 & 326.4199 & 279.4336 & 355.1657 & 348.8613 & 243.6538 & 385.7041 \\
\hline EGU & 0.0309 & 0.0099 & 0.0238 & 0.0000 & & 251.2966 & 169.9370 & 299.3431 & 235.1849 & 194.7626 & 364.9853 \\
\hline GUB & 0.0000 & 0.0000 & 0.0000 & 0.0000 & 0.0005 & & 238.6009 & 323.5085 & 251.3156 & 213.7887 & 354.1472 \\
\hline HAT & 0.0440 & 0.0886 & 0.0120 & 0.0000 & 0.9480 & 0.0109 & & 335.4467 & 230.7371 & 198.6350 & 332.5014 \\
\hline HUT & 0.0000 & 0.0000 & 0.0001 & 0.0000 & 0.0000 & 0.0000 & 0.0000 & & 301.1588 & 210.3549 & 376.5460 \\
\hline JAM & 0.7290 & 0.1866 & 0.0002 & 0.0000 & 0.0122 & 0.0006 & 0.0462 & 0.0000 & & 195.1255 & 333.7593 \\
\hline KEN & 0.1050 & 0.0247 & 0.0916 & 0.0003 & 0.1682 & 0.0193 & 0.2982 & 0.0516 & 0.2052 & & 306.9289 \\
\hline MAD & 0.0000 & 0.0000 & 0.0000 & 0.0000 & 0.0000 & 0.0000 & 0.0000 & 0.0000 & 0.0000 & 0.0000 & \\
\hline MOZ & 0.0000 & 0.0000 & 0.0000 & 0.0005 & 0.0000 & 0.0000 & 0.0000 & 0.0000 & 0.0000 & 0.0035 & 0.0000 \\
\hline NAM & 0.0000 & 0.0000 & 0.0000 & 0.0000 & 0.0000 & 0.0000 & 0.0000 & 0.0000 & 0.0000 & 0.0000 & 0.0000 \\
\hline NWP & 0.3478 & 0.0142 & 0.0000 & 0.0000 & 0.0440 & 0.0009 & 0.0847 & 0.0000 & 0.1261 & 0.0754 & 0.0000 \\
\hline TAN & 0.0000 & 0.0000 & 0.0000 & 0.0000 & 0.0000 & 0.0000 & 0.0000 & 0.0000 & 0.0000 & 0.0365 & 0.0000 \\
\hline TUT & 0.0000 & 0.0000 & 0.0000 & 0.0000 & 0.0000 & 0.0000 & 0.0000 & 0.0000 & 0.0000 & 0.0000 & 0.0000 \\
\hline UGA & 0.0000 & 0.0000 & 0.0000 & 0.0000 & 0.0000 & 0.0000 & 0.0000 & 0.0000 & 0.0000 & 0.0003 & 0.0000 \\
\hline $\mathrm{CCS}$ & 0.0000 & 0.0000 & 0.0000 & 0.0000 & 0.0000 & 0.0000 & 0.0000 & 0.0000 & 0.0000 & 0.0000 & 0.0000 \\
\hline JAP & 0.0000 & 0.0000 & 0.0000 & 0.0000 & 0.0000 & 0.0000 & 0.0000 & 0.0000 & 0.0000 & 0.0000 & 0.0000 \\
\hline KOR & 0.0000 & 0.0000 & 0.0000 & 0.0000 & 0.0000 & 0.0000 & 0.0000 & 0.0000 & 0.0000 & 0.0000 & 0.0000 \\
\hline MAL & 0.0000 & 0.0000 & 0.0000 & 0.0000 & 0.0000 & 0.0000 & 0.0000 & 0.0000 & 0.0000 & 0.0000 & 0.0000 \\
\hline PHI & 0.0000 & 0.0000 & 0.0000 & 0.0000 & 0.0000 & 0.0000 & 0.0000 & 0.0000 & 0.0000 & 0.0000 & 0.0000 \\
\hline CSH & 0.0000 & 0.0000 & 0.0000 & 0.0000 & 0.0000 & 0.0000 & 0.0000 & 0.0000 & 0.0000 & 0.0000 & 0.0000 \\
\hline CAR & 0.0000 & 0.0000 & 0.0000 & 0.0000 & 0.0000 & 0.0000 & 0.0000 & 0.0000 & 0.0000 & 0.0000 & 0.0000 \\
\hline MEX & 0.0000 & 0.0000 & 0.0000 & 0.0000 & 0.0000 & 0.0000 & 0.0000 & 0.0000 & 0.0000 & 0.0000 & 0.0000 \\
\hline CSR & 0.0000 & 0.0000 & 0.0000 & 0.0000 & 0.0000 & 0.0000 & 0.0000 & 0.0000 & 0.0000 & 0.0000 & 0.0000 \\
\hline HIS & 0.0000 & 0.0000 & 0.0000 & 0.0000 & 0.0000 & 0.0000 & 0.0000 & 0.0000 & 0.0000 & 0.0000 & 0.0000 \\
\hline MAR & 0.0000 & 0.0000 & 0.0000 & 0.0000 & 0.0000 & 0.0000 & 0.0000 & 0.0000 & 0.0000 & 0.0000 & 0.0000 \\
\hline MET & 0.0000 & 0.0000 & 0.0000 & 0.0000 & 0.0000 & 0.0000 & 0.0000 & 0.0000 & 0.0000 & 0.0000 & 0.0000 \\
\hline PRA & 0.0000 & 0.0000 & 0.0000 & 0.0000 & 0.0000 & 0.0000 & 0.0000 & 0.0000 & 0.0000 & 0.0000 & 0.0000 \\
\hline BRA & 0.0000 & 0.0000 & 0.0000 & 0.0000 & 0.0000 & 0.0000 & 0.0000 & 0.0000 & 0.0000 & 0.0000 & 0.0000 \\
\hline AND & 0.0000 & 0.0000 & 0.0000 & 0.0000 & 0.0000 & 0.0000 & 0.0000 & 0.0000 & 0.0000 & 0.0000 & 0.0000 \\
\hline BLR & 0.0000 & 0.0000 & 0.0000 & 0.0000 & 0.0000 & 0.0000 & 0.0000 & 0.0000 & 0.0000 & 0.0000 & 0.0000 \\
\hline CAU & 0.0000 & 0.0000 & 0.0000 & 0.0000 & 0.0000 & 0.0000 & 0.0000 & 0.0000 & 0.0000 & 0.0000 & 0.0000 \\
\hline FLE & 0.0000 & 0.0000 & 0.0000 & 0.0000 & 0.0000 & 0.0000 & 0.0000 & 0.0000 & 0.0000 & 0.0000 & 0.0000 \\
\hline POL & 0.0000 & 0.0000 & 0.0000 & 0.0000 & 0.0000 & 0.0000 & 0.0000 & 0.0000 & 0.0000 & 0.0000 & 0.0000 \\
\hline SPN & 0.0000 & 0.0000 & 0.0000 & 0.0000 & 0.0000 & 0.0000 & 0.0000 & 0.0000 & 0.0000 & 0.0000 & 0.0000 \\
\hline
\end{tabular}

Refer to Table 1 for key to abbreviations

Pair-wise population comparisons yielding statistically insignificant differences both before (in bold) and after (in italics) applying the Bonferroni correction

Table 8: G-test 


\begin{tabular}{|c|c|c|c|c|c|c|c|c|c|c|c|}
\hline & MOZ & NAM & NWP & TAN & TUT & UGA & CCS & JAP & KOR & MAL & PHI \\
\hline US1 & 369.3774 & 504.0229 & 211.5335 & 370.5138 & 487.7301 & 436.9200 & 1321.3734 & 1815.1772 & 1571.8273 & 1145.4186 & 956.6950 \\
\hline US2 & 336.3618 & 544.4847 & 260.9949 & 400.2196 & 523.5327 & 483.2588 & 1612.5165 & 2444.9363 & 1978.6023 & 1474.4789 & 1150.3481 \\
\hline ANG & 334.9184 & 290.9848 & 297.1790 & 327.6299 & 378.0364 & 468.1497 & 1319.5687 & 1743.0122 & 1547.0536 & 1230.0575 & 994.3469 \\
\hline SAF & 246.7927 & 419.0391 & 414.8787 & 330.5381 & 440.1677 & 510.8528 & 1347.2368 & 1787.0132 & 1613.6172 & 1294.1763 & 1088.1547 \\
\hline EGU & 317.1875 & 352.9726 & 239.0506 & 334.4801 & 407.6942 & 456.9542 & 1410.5985 & 1908.0112 & 1703.4598 & 1264.7218 & 1045.0815 \\
\hline GUB & 312.9706 & 394.3167 & 265.0812 & 321.6954 & 440.6943 & 416.2239 & 1101.0726 & 1417.4559 & 1327.3401 & 1044.8998 & 898.3178 \\
\hline HAT & 297.7143 & 337.0298 & 236.7576 & 308.1873 & 414.9631 & 416.9154 & 1258.7485 & 1702.6776 & 1540.2103 & 1153.0020 & 987.1977 \\
\hline HUT & 352.6128 & 462.6141 & 344.6294 & 333.5834 & 361.3040 & 328.6649 & 1243.8484 & 1588.8331 & 1444.2677 & 1138.2894 & 1010.2482 \\
\hline JAM & 305.3622 & 419.8413 & 228.4719 & 299.7603 & 405.2145 & 367.2077 & 1105.7494 & 1451.3796 & 1325.4003 & 987.2772 & 861.8778 \\
\hline KEN & 225.2373 & 299.9806 & 213.9525 & 227.2508 & 297.4595 & 241.7360 & 965.3720 & 1132.9982 & 1112.2170 & 886.5229 & 784.0193 \\
\hline MAD & 371.9299 & 506.4148 & 421.1929 & 437.2512 & 461.2226 & 501.2495 & 708.5034 & 868.1801 & 857.8117 & 625.9515 & 527.1189 \\
\hline MOZ & & 486.0583 & 408.3094 & 283.4961 & 523.5334 & 519.5912 & 1516.8990 & 1978.8864 & 1779.2944 & 1456.1948 & 1166.9080 \\
\hline NAM & 0.0000 & & 548.6736 & 534.3686 & 634.0131 & 622.8959 & 1964.9546 & 2927.8337 & 2514.9470 & 1966.7240 & 1467.2649 \\
\hline NWP & 0.0000 & 0.0000 & & 450.5210 & 484.4134 & 462.1212 & 1640.0826 & 2419.7532 & 2044.1027 & 1505.4727 & 1227.9337 \\
\hline TAN & 0.0001 & 0.0000 & 0.0000 & & 481.9146 & 527.5989 & 1851.1737 & 2691.5598 & 2241.6169 & 1783.1946 & 1353.2587 \\
\hline TUT & 0.0000 & 0.0000 & 0.0000 & 0.0000 & & 537.8627 & 1533.4893 & 1974.8872 & 1805.7122 & 1471.2946 & 1165.1117 \\
\hline UGA & 0.0000 & 0.0000 & 0.0000 & 0.0000 & 0.0000 & & 1159.6693 & 1464.1675 & 1357.8638 & 1083.6028 & 974.2226 \\
\hline $\mathrm{CCS}$ & 0.0000 & 0.0000 & 0.0000 & 0.0000 & 0.0000 & 0.0000 & & 431.2647 & 353.6910 & 432.0402 & 356.7864 \\
\hline JAP & 0.0000 & 0.0000 & 0.0000 & 0.0000 & 0.0000 & 0.0000 & 0.0000 & & 293.7658 & 741.7219 & 497.2908 \\
\hline KOR & 0.0000 & 0.0000 & 0.0000 & 0.0000 & 0.0000 & 0.0000 & 0.0000 & 0.0000 & & 636.6891 & 459.4520 \\
\hline MAL & 0.0000 & 0.0000 & 0.0000 & 0.0000 & 0.0000 & 0.0000 & 0.0000 & 0.0000 & 0.0000 & & 289.0813 \\
\hline PHI & 0.0000 & 0.0000 & 0.0000 & 0.0000 & 0.0000 & 0.0000 & 0.0000 & 0.0000 & 0.0000 & 0.0000 & \\
\hline CSH & 0.0000 & 0.0000 & 0.0000 & 0.0000 & 0.0000 & 0.0000 & 0.0000 & 0.0000 & 0.0000 & 0.0000 & 0.0000 \\
\hline CAR & 0.0000 & 0.0000 & 0.0000 & 0.0000 & 0.0000 & 0.0000 & 0.0000 & 0.0000 & 0.0000 & 0.0000 & 0.0000 \\
\hline MEX & 0.0000 & 0.0000 & 0.0000 & 0.0000 & 0.0000 & 0.0000 & 0.0000 & 0.0000 & 0.0000 & 0.0000 & 0.0000 \\
\hline CSR & 0.0000 & 0.0000 & 0.0000 & 0.0000 & 0.0000 & 0.0000 & 0.0000 & 0.0000 & 0.0000 & 0.0000 & 0.0000 \\
\hline HIS & 0.0000 & 0.0000 & 0.0000 & 0.0000 & 0.0000 & 0.0000 & 0.0000 & 0.0000 & 0.0000 & 0.0000 & 0.0000 \\
\hline MAR & 0.0000 & 0.0000 & 0.0000 & 0.0000 & 0.0000 & 0.0000 & 0.0000 & 0.0000 & 0.0000 & 0.0000 & 0.0000 \\
\hline MET & 0.0000 & 0.0000 & 0.0000 & 0.0000 & 0.0000 & 0.0000 & 0.0000 & 0.0000 & 0.0000 & 0.0000 & 0.0000 \\
\hline PRA & 0.0000 & 0.0000 & 0.0000 & 0.0000 & 0.0000 & 0.0000 & 0.0000 & 0.0000 & 0.0000 & 0.0000 & 0.0000 \\
\hline BRA & 0.0000 & 0.0000 & 0.0000 & 0.0000 & 0.0000 & 0.0000 & 0.0000 & 0.0000 & 0.0000 & 0.0000 & 0.0000 \\
\hline AND & 0.0000 & 0.0000 & 0.0000 & 0.0000 & 0.0000 & 0.0000 & 0.0000 & 0.0000 & 0.0000 & 0.0000 & 0.0000 \\
\hline BLR & 0.0000 & 0.0000 & 0.0000 & 0.0000 & 0.0000 & 0.0000 & 0.0000 & 0.0000 & 0.0000 & 0.0000 & 0.0000 \\
\hline CAU & 0.0000 & 0.0000 & 0.0000 & 0.0000 & 0.0000 & 0.0000 & 0.0000 & 0.0000 & 0.0000 & 0.0000 & 0.0000 \\
\hline FLE & 0.0000 & 0.0000 & 0.0000 & 0.0000 & 0.0000 & 0.0000 & 0.0000 & 0.0000 & 0.0000 & 0.0000 & 0.0000 \\
\hline POL & 0.0000 & 0.0000 & 0.0000 & 0.0000 & 0.0000 & 0.0000 & 0.0000 & 0.0000 & 0.0000 & 0.0000 & 0.0000 \\
\hline SPN & 0.0000 & 0.0000 & 0.0000 & 0.0000 & 0.0000 & 0.0000 & 0.0000 & 0.0000 & 0.0000 & 0.0000 & 0.0000 \\
\hline
\end{tabular}

Refer to Table 1 for key to abbreviations

Pair-wise population comparisons yielding statistically insignificant differences both before (in bold) and after (in italics) applying the Bonferroni correction

\section{Table 8: G-test (Cont'd)}




\begin{tabular}{|c|c|c|c|c|c|c|c|c|c|}
\hline & CSH & CAR & MEX & CSR & HIS & MAR & MET & PRA & BRA \\
\hline US1 & 1366.2150 & 720.6636 & 1018.0546 & 1026.5992 & 681.2700 & 717.2574 & 1314.0172 & 618.4646 & 503.5044 \\
\hline US2 & 1668.1620 & 924.5727 & 1335.5060 & 1435.1771 & 863.0743 & 943.2885 & 1650.6353 & 830.7430 & 673.8070 \\
\hline ANG & 1340.3058 & 893.2872 & 1170.4983 & 1226.6724 & 855.5585 & 906.1671 & 1417.8441 & 785.2439 & 656.7479 \\
\hline SAF & 1417.8231 & 951.1696 & 1255.0952 & 1228.6013 & 935.6351 & 1016.5126 & 1419.9254 & 881.2035 & 788.1117 \\
\hline EGU & 1449.3634 & 853.7404 & 1166.7423 & 1237.9307 & 842.0637 & 892.4207 & 1452.4832 & 754.5046 & 653.2888 \\
\hline GUB & 1120.6151 & 669.1605 & 942.4453 & 929.3496 & 685.0397 & 746.6887 & 1180.8689 & 649.6805 & 563.4520 \\
\hline HAT & 1291.3613 & 781.3412 & 1056.8923 & 1114.7231 & 778.7883 & 821.7631 & 1295.0150 & 726.1169 & 589.4932 \\
\hline HUT & 1298.2859 & 803.4444 & 1042.8290 & 1083.9535 & 805.6285 & 805.2376 & 1316.9272 & 691.0500 & 647.6494 \\
\hline JAM & 1130.8444 & 616.8490 & 880.0184 & 918.5863 & 637.9739 & 668.6646 & 1130.4785 & 577.1983 & 482.6524 \\
\hline KEN & 959.9223 & 577.6357 & 754.5463 & 736.7450 & 576.3684 & 587.2698 & 931.8091 & 535.9065 & 430.8232 \\
\hline MAD & 775.9848 & 553.3486 & 678.7587 & 725.1730 & 529.5678 & 579.8524 & 803.1285 & 558.0521 & 532.5978 \\
\hline MOZ & 1575.8204 & 989.1948 & 1346.6300 & 1351.3932 & 956.1947 & 1024.2223 & 1573.4152 & 905.7759 & 824.1256 \\
\hline NAM & 2218.7803 & 1549.8578 & 1926.5803 & 2248.2058 & 1367.1688 & 1593.1064 & 2180.9568 & 1340.3010 & 1211.3700 \\
\hline NWP & 1753.9027 & 916.2577 & 1304.4275 & 1401.6000 & 834.9598 & 918.8211 & 1625.9066 & 753.7961 & 658.0684 \\
\hline TAN & 1953.3273 & 1328.6235 & 1714.4022 & 1863.9269 & 1228.1897 & 1356.3922 & 2000.8979 & 1146.5701 & 1061.1766 \\
\hline TUT & 1633.0477 & 1071.8092 & 1358.7449 & 1459.8461 & 1011.6371 & 1045.1583 & 1561.3545 & 976.0688 & 926.4795 \\
\hline UGA & 1209.6449 & 863.1320 & 1019.6701 & 1109.8484 & 778.4283 & 827.5852 & 1233.6215 & 809.3176 & 715.9099 \\
\hline CCS & 279.7270 & 1125.1412 & 1056.0265 & 1340.8743 & 849.0118 & 1002.9550 & 1209.4363 & 1094.1124 & 1297.0906 \\
\hline JAP & 487.8763 & 1469.3901 & 1516.2504 & 1839.5123 & 972.6217 & 1249.6879 & 1583.5695 & 1436.7079 & 1865.7714 \\
\hline KOR & 344.5786 & 1187.6215 & 1165.9557 & 1402.4498 & 844.1308 & 1037.3330 & 1330.2899 & 1180.2168 & 1468.2697 \\
\hline MAL & 472.2081 & 918.8443 & 877.5515 & 1212.1229 & 618.0319 & 803.8587 & 1130.5956 & 867.9807 & 1118.8350 \\
\hline PHI & 377.1124 & 877.9362 & 854.0881 & 1016.2513 & 674.0782 & 786.16370 & 1106.5436 & 830.0125 & 957.3215 \\
\hline CSH & & 1131.8489 & 1023.6318 & 1295.5643 & 789.0430 & 979.0354 & 1224.3311 & 1062.3735 & 1312.8925 \\
\hline CAR & 0.0000 & & 434.5750 & 291.5373 & 206.2046 & 194.9020 & 757.1349 & 323.2320 & 335.2340 \\
\hline MEX & 0.0000 & 0.0000 & & 545.4443 & 261.7632 & 378.3874 & 583.8787 & 574.1693 & 686.5186 \\
\hline CSR & 0.0000 & 0.0000 & 0.0000 & & 248.0351 & 317.3796 & 779.3468 & 519.6517 & 604.9189 \\
\hline HIS & 0.0000 & 0.1002 & 0.0023 & 0.0001 & & 220.7598 & 442.0474 & 304.3576 & 352.9807 \\
\hline MAR & 0.0000 & 0.4610 & 0.0000 & 0.0000 & 0.0103 & & 611.7493 & 369.8294 & 340.7615 \\
\hline MET & 0.0000 & 0.0000 & 0.0000 & 0.0000 & 0.0000 & 0.0000 & & 941.7846 & 1130.1987 \\
\hline PRA & 0.0000 & 0.0000 & 0.0000 & 0.0000 & 0.0000 & 0.0000 & 0.0000 & & 353.1628 \\
\hline BRA & 0.0000 & 0.0000 & 0.0000 & 0.0000 & 0.0000 & 0.0000 & 0.0000 & 0.0000 & \\
\hline AND & 0.0000 & 0.0000 & 0.0000 & 0.0000 & 0.0000 & 0.0000 & 0.0000 & 0.0000 & 0.0000 \\
\hline BLR & 0.0000 & 0.0000 & 0.0000 & 0.0000 & 0.0000 & 0.0000 & 0.0000 & 0.0000 & 0.0000 \\
\hline CAU & 0.0000 & 0.0000 & 0.0000 & 0.0000 & 0.0000 & 0.0000 & 0.0000 & 0.0000 & 0.0000 \\
\hline FLE & 0.0000 & 0.0000 & 0.0000 & 0.0000 & 0.0000 & 0.0000 & 0.0000 & 0.0000 & 0.0000 \\
\hline POL & 0.0000 & 0.0000 & 0.0000 & 0.0000 & 0.0000 & 0.0000 & 0.0000 & 0.0000 & 0.0000 \\
\hline SPN & 0.0000 & 0.0000 & 0.0000 & 0.0000 & 0.0000 & 0.0000 & 0.0000 & 0.0000 & 0.0000 \\
\hline
\end{tabular}

Refer to Table 1 for key to abbreviations

Pair-wise population comparisons yielding statistically insignificant differences both before (in bold) and after (in italics) applying the Bonferroni correction

\section{Table 8: G-test (Cont'd)}




\begin{tabular}{|c|c|c|c|c|c|c|}
\hline & AND & BLR & CAU & FLE & POL & SPN \\
\hline US1 & 681.6089 & 949.8528 & 1044.4484 & 1018.1482 & 1313.8867 & 1147.5273 \\
\hline US2 & 873.9698 & 1193.3600 & 1413.5024 & 1345.8695 & 1775.0813 & 1548.8081 \\
\hline ANG & 883.2368 & 1118.5775 & 1275.2731 & 1231.9263 & 1470.5598 & 1355.2976 \\
\hline SAF & 946.0394 & 1259.1360 & 1347.4409 & 1312.3367 & 1584.3025 & 1425.7090 \\
\hline EGU & 831.1876 & 1117.4847 & 1275.5774 & 1177.8361 & 1488.0153 & 1311.7319 \\
\hline GUB & 760.4780 & 960.4456 & 1036.7103 & 1000.3499 & 1215.0681 & 1098.9459 \\
\hline HAT & 806.1568 & 1076.9105 & 1138.1791 & 1094.1213 & 1371.2069 & 1187.7039 \\
\hline HUT & 803.9218 & 982.7222 & 1098.8838 & 1081.3782 & 1249.1531 & 1185.1730 \\
\hline JAM & 666.4562 & 912.5247 & 978.4950 & 948.8259 & 1174.8984 & 1039.3077 \\
\hline KEN & 619.4303 & 823.0012 & 835.1530 & 827.1734 & 983.0800 & 891.3480 \\
\hline MAD & 656.8502 & 799.5281 & 863.2474 & 849.9046 & 943.9898 & 908.0583 \\
\hline MOZ & 970.4476 & 1301.5133 & 1439.9630 & 1420.3245 & 1726.0402 & 1557.8480 \\
\hline NAM & 1316.3779 & 1716.7960 & 2128.6575 & 1949.6779 & 2491.4854 & 2229.7070 \\
\hline NWP & 871.0754 & 1150.2979 & 1319.9277 & 1277.3502 & 1710.0082 & 1462.1938 \\
\hline TAN & 1203.8531 & 1595.0778 & 1900.5359 & 1805.7930 & 2265.0481 & 2074.0374 \\
\hline TUT & 976.5187 & 1242.6163 & 1480.4247 & 1403.4790 & 1682.7332 & 1564.5051 \\
\hline UGA & 845.9526 & 1026.6661 & 1078.6869 & 1088.3894 & 1281.5101 & 1225.9478 \\
\hline CCS & 889.4481 & 1176.4991 & 1356.3972 & 1272.7761 & 1577.0469 & 1384.5605 \\
\hline JAP & 1030.0175 & 1547.7587 & 1952.4344 & 1806.0680 & 2485.0547 & 2096.0361 \\
\hline KOR & 891.8792 & 1247.3016 & 1462.3920 & 1391.4364 & 1799.3508 & 1547.2969 \\
\hline MAL & 695.2513 & 972.6280 & 1192.6473 & 1130.7333 & 1423.9149 & 1216.8807 \\
\hline PHI & 711.6191 & 914.1631 & 1044.2216 & 990.5612 & 1174.5063 & 1085.7383 \\
\hline CSH & 874.6797 & 1201.5341 & 1388.6058 & 1331.6224 & 1709.8162 & 1476.5314 \\
\hline CAR & 351.6024 & 551.6054 & 503.3499 & 533.7566 & 753.9863 & 527.7271 \\
\hline MEX & 493.6120 & 726.5411 & 783.9633 & 740.4211 & 1093.4510 & 840.4451 \\
\hline CSR & 491.1570 & 716.8347 & 726.0587 & 752.0983 & 1151.3206 & 829.2176 \\
\hline HIS & 378.2934 & 507.0346 & 451.7975 & 484.1142 & 674.2834 & 510.1093 \\
\hline MAR & 303.9688 & 479.3408 & 423.3698 & 457.7372 & 618.5443 & 461.2663 \\
\hline MET & 910.9257 & 1208.4436 & 1256.2998 & 1165.7612 & 1651.3641 & 1436.8680 \\
\hline PRA & 376.5916 & 593.3844 & 509.8419 & 543.5773 & 734.4340 & 528.5404 \\
\hline BRA & 379.7395 & 570.6100 & 528.9551 & 564.9875 & 784.5393 & 570.0999 \\
\hline AND & & 259.4031 & 254.3532 & 240.6117 & 295.3663 & 191.7305 \\
\hline BLR & 0.0000 & & 286.8939 & 272.8293 & 202.3072 & 336.5193 \\
\hline CAU & 0.0000 & 0.0000 & & 229.5211 & 276.5790 & 287.8519 \\
\hline FLE & 0.0000 & 0.0000 & 0.0030 & & 330.2069 & 319.8255 \\
\hline POL & 0.0000 & 0.0602 & 0.0000 & 0.0000 & & 387.8344 \\
\hline SPN & 0.0329 & 0.0000 & 0.0000 & 0.0000 & 0.0000 & \\
\hline
\end{tabular}

Refer to Table 1 for key to abbreviations

Pair-wise population comparisons yielding statistically insignificant differences both before (in bold) and after (in italics) applying the Bonferroni correction

Table 8: G-test (Cont'd) 
Haiti

\begin{tabular}{lccc} 
& $\mathrm{m}_{\mathrm{L}}$ & $\mathrm{m}_{\mathrm{Y}}$ & $\mathrm{m}_{\mathrm{RH}}$ \\
Parental Groups & & & \\
\hline African & $0.955 \pm 0.022^{\mathrm{a}}$ & $0.778177 \pm 0.122908^{\mathrm{b}}$ & $1.0021 \pm 0.0359^{\mathrm{b}}$ \\
East Asian & $0.003 \pm 0.025^{\mathrm{a}}$ & $-0.0758699 \pm 0.193892^{\mathrm{b}}$ & $-0.0219 \pm 0.0347^{\mathrm{b}}$ \\
European & $0.043 \pm 0.027^{\mathrm{a}}$ & $0.297693 \pm 0.252631^{\mathrm{b}}$ & $0.0198 \pm 0.0527^{\mathrm{b}}$ \\
\hline
\end{tabular}

Jamaica

\begin{tabular}{lccc} 
& $\mathrm{m}_{\mathrm{L}}$ & $\mathrm{m}_{\mathrm{Y}}$ & $\mathrm{m}_{\mathrm{RH}}$ \\
Parental Groups & & & \\
\hline African & $0.783 \pm 0.020^{\mathrm{a}}$ & $0.764788 \pm 0.126145^{\mathrm{b}}$ & $0.8212 \pm 0.0657^{\mathrm{b}}$ \\
East Asian & $0.057 \pm 0.022^{\mathrm{a}}$ & $0.0564919 \pm 0.179226^{\mathrm{b}}$ & $0.0373 \pm 0.0423^{\mathrm{b}}$ \\
European & $0.160 \pm 0.024^{\mathrm{a}}$ & $0.17872 \pm 0.239307^{\mathrm{b}}$ & $0.1415 \pm 0.0657^{\mathrm{b}}$ \\
\hline
\end{tabular}

New Providence (Bahamas)

\begin{tabular}{lccc} 
& $\mathrm{m}_{\mathrm{L}}$ & $\mathrm{m}_{\mathrm{Y}}$ & $\mathrm{m}_{\mathrm{RH}}$ \\
Parental Groups & & & \\
\hline African & $0.780 \pm 0.017^{\mathrm{a}}$ & $0.783536 \pm 0.105421^{\mathrm{b}}$ & $0.8366 \pm 0.0243^{\mathrm{b}}$ \\
East Asian & $0.000 \pm 0.019^{\mathrm{a}}$ & $-0.0707197 \pm 0.148^{\mathrm{b}}$ & $-0.0547 \pm 0.0315^{\mathrm{b}}$ \\
European & $0.220 \pm 0.021^{\mathrm{a}}$ & $0.287184 \pm 0.186574^{\mathrm{b}}$ & $0.2181 \pm 0.0379^{\mathrm{b}}$ \\
\hline
\end{tabular}

US African American 1 (Minnesota)

\begin{tabular}{|c|c|c|c|}
\hline Parental Groups & $\mathrm{m}_{\mathrm{L}}$ & $\mathrm{m}_{\mathrm{Y}}$ & $\mathrm{m}_{\mathrm{RH}}$ \\
\hline African & $0.780 \pm 0.019^{\mathrm{a}}$ & $0.697312 \pm 0.0705579^{b}$ & $0.8276 \pm 0.0440^{\mathrm{b}}$ \\
\hline East Asian & $0.004 \pm 0.022^{\mathrm{a}}$ & $-0.0609425 \pm 0.217413^{b}$ & $-0.0215 \pm 0.0443^{\mathrm{b}}$ \\
\hline European & $0.216 \pm 0.024^{\mathrm{a}}$ & $0.36363 \pm 0.230357^{\mathrm{b}}$ & $0.1939 \pm 0.0550^{\mathrm{b}}$ \\
\hline
\end{tabular}

US African American 2 (General)

\begin{tabular}{lccc} 
& $\mathrm{m}_{\mathrm{L}}$ & $\mathrm{m}_{\mathrm{Y}}$ & $\mathrm{m}_{\mathrm{RH}}$ \\
Parental Groups & & & \\
\hline African & $0.782 \pm 0.016^{\mathrm{a}}$ & $0.711886 \pm 0.0651698^{\mathrm{b}}$ & $0.8228 \pm 0.0297^{\mathrm{b}}$ \\
East Asian & $0.030 \pm 0.018^{\mathrm{a}}$ & $-0.0178861 \pm 0.158548^{\mathrm{b}}$ & $0.0101 \pm 0.0210^{\mathrm{b}}$ \\
European & $0.188 \pm 0.019^{\mathrm{a}}$ & $0.306 \pm 0.188193^{\mathrm{b}}$ & $0.1671 \pm 0.0360^{\mathrm{b}}$ \\
\hline
\end{tabular}

Table 9: Admixture analyses for the New World African populations 
Supporting Information Figure 1: Histograms comparing allele frequency data for Haiti and Jamaica per STR locus
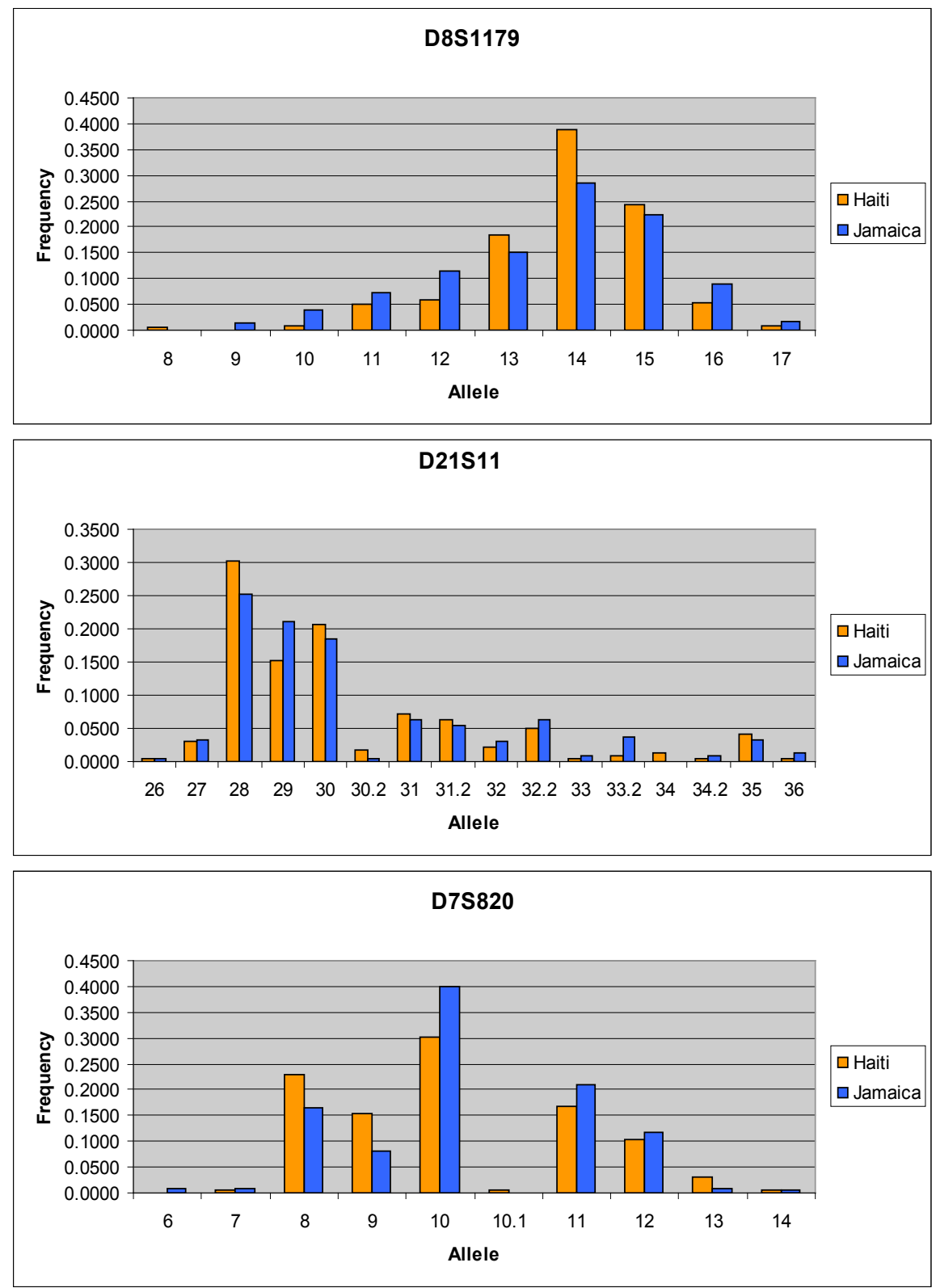

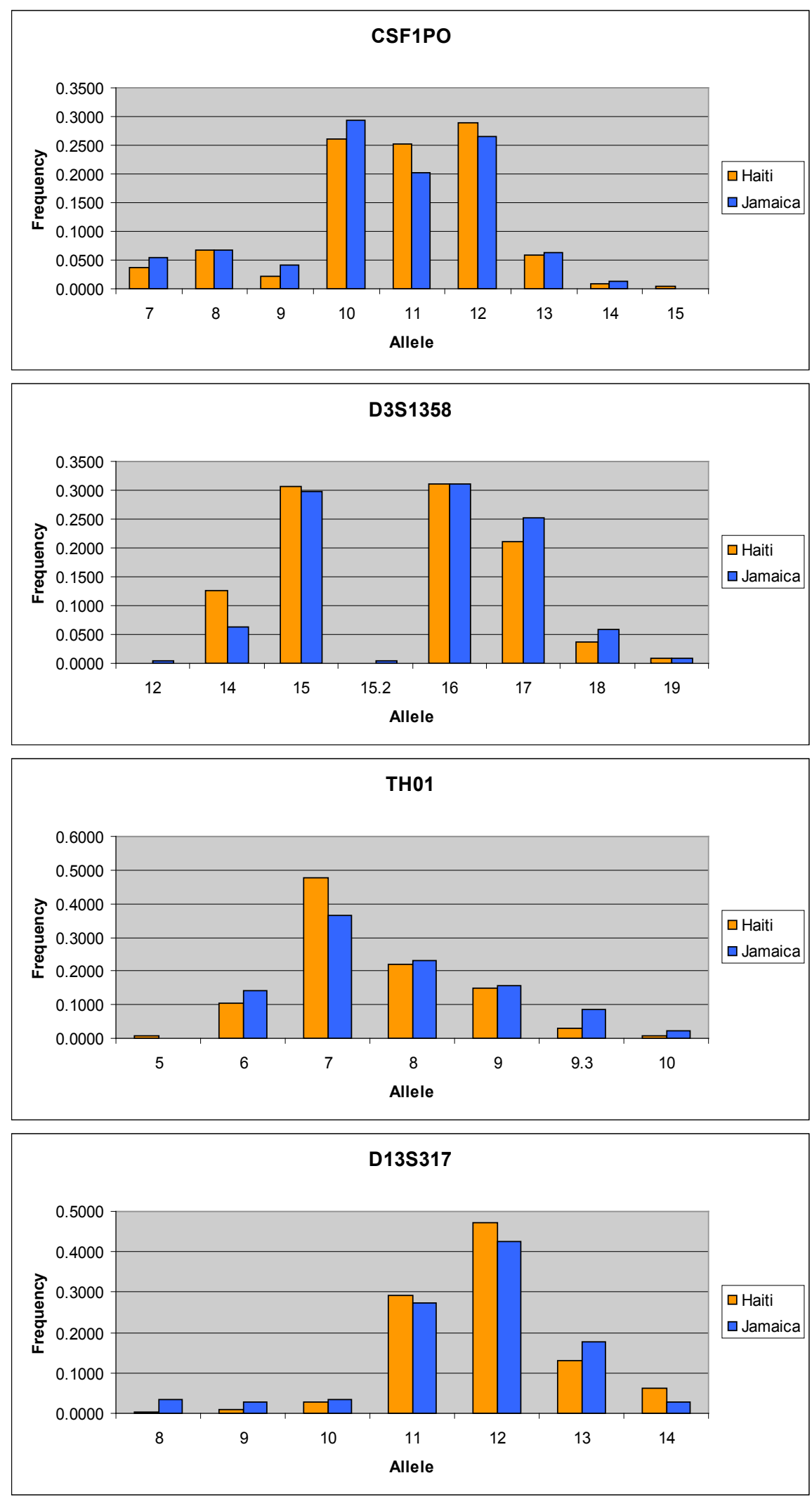

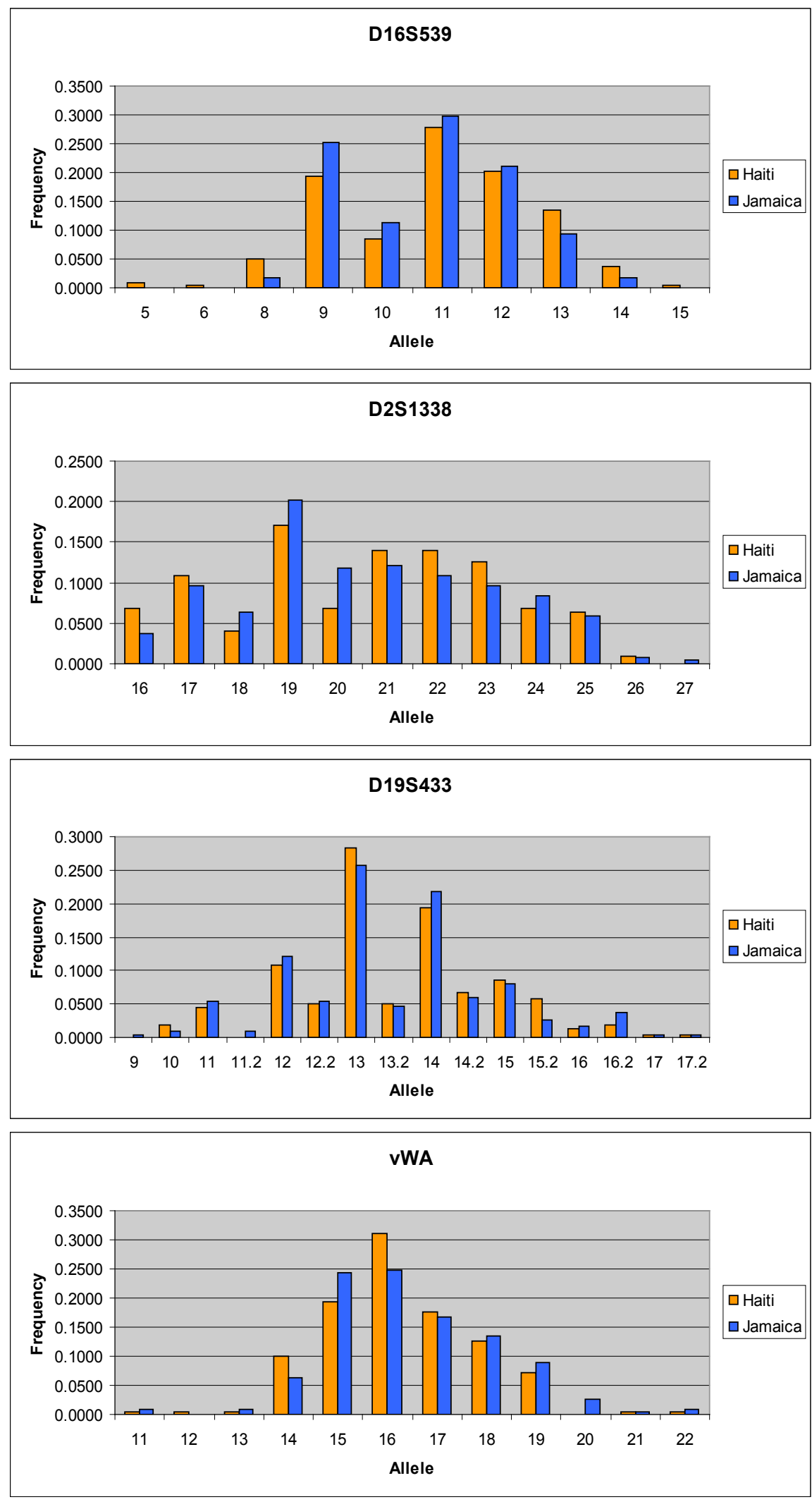

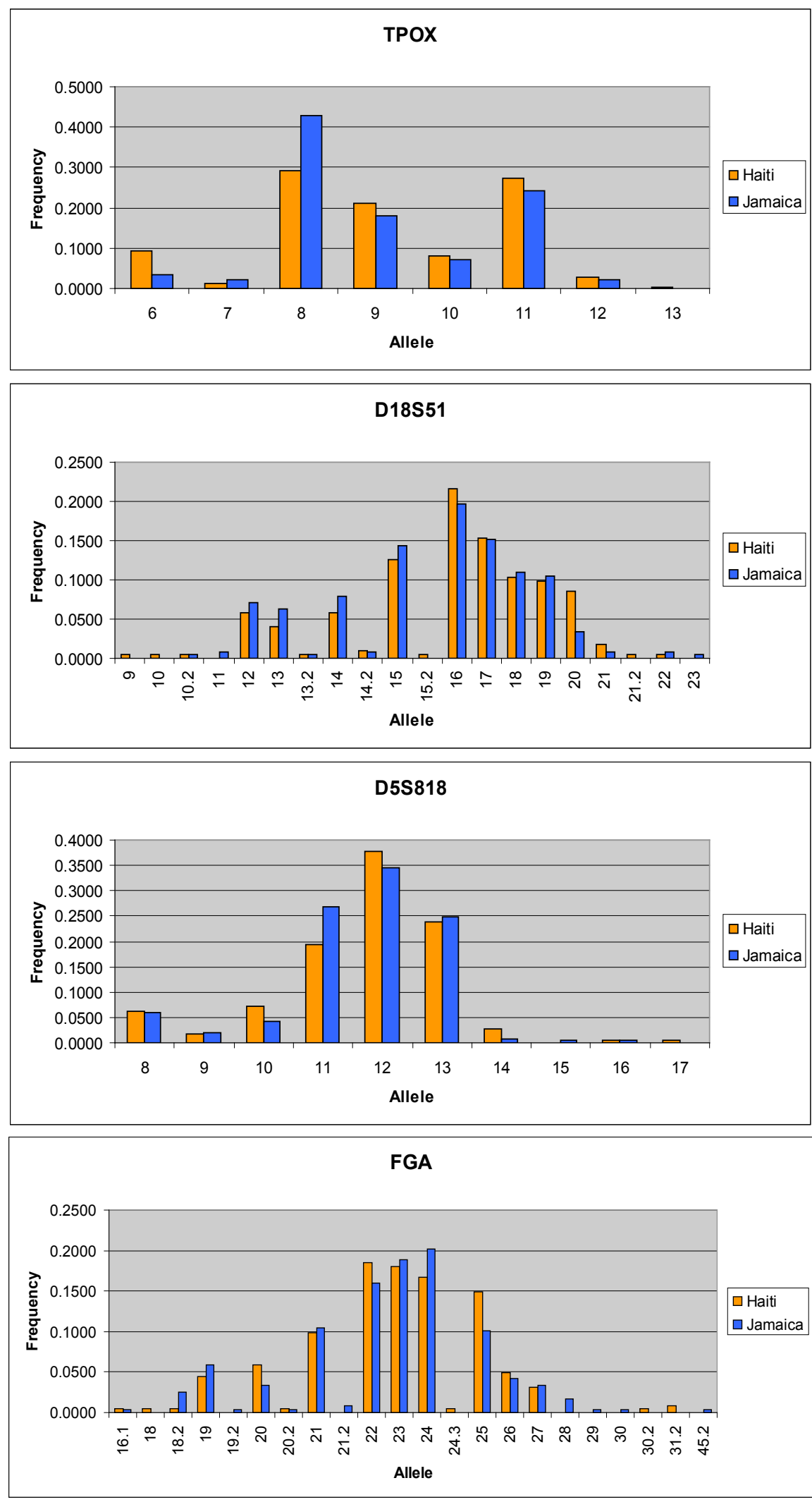
Supporting Information Figure 2: Multidimensional Scaling Plot (Stress $=\mathbf{0 . 1 3 1 2 4})$ of all populations at 13 loci

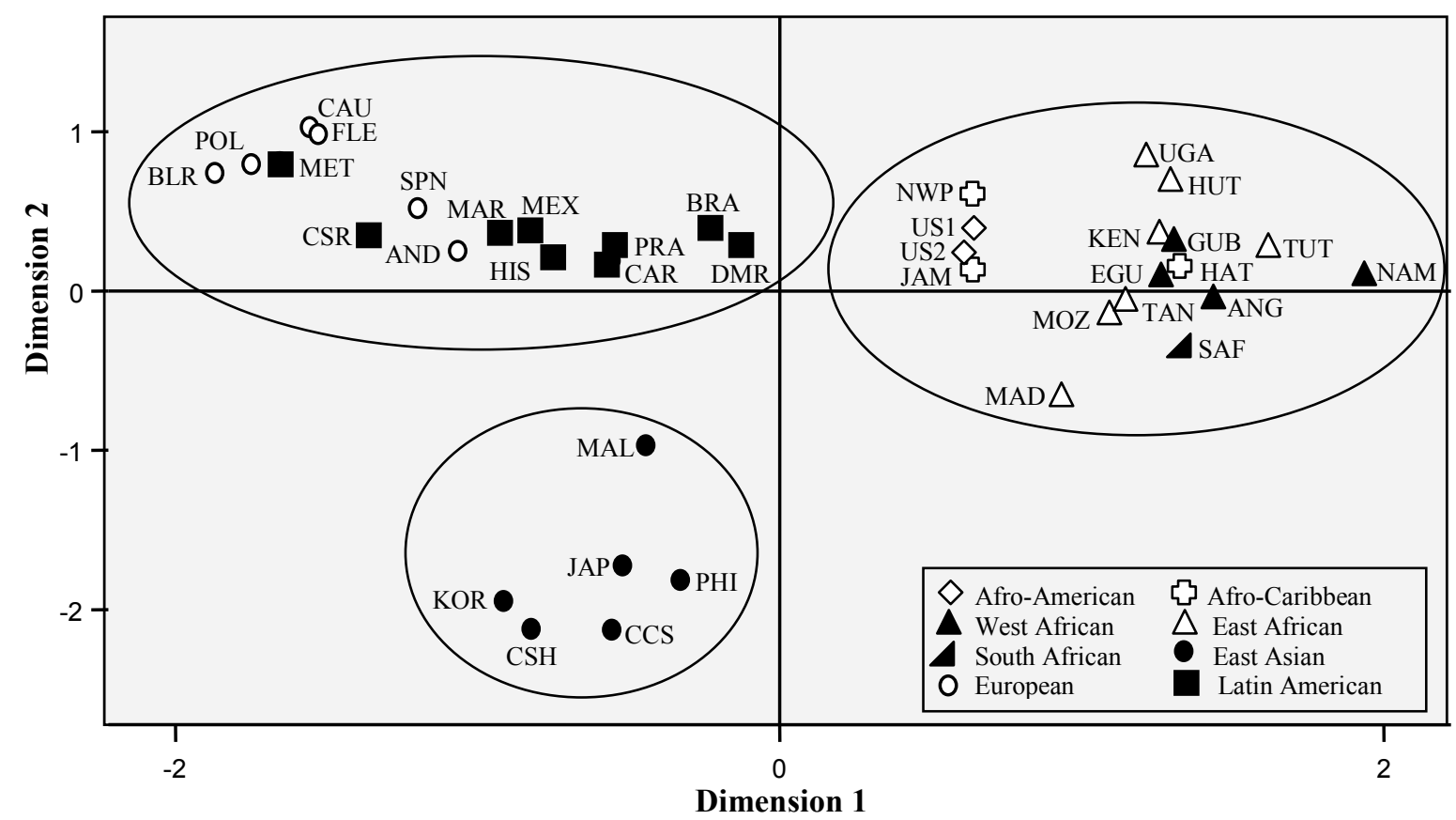


Supporting Information Figure 3: Structure bar plots assuming three and four ancestral populations $(K=3$ and $K=4)$

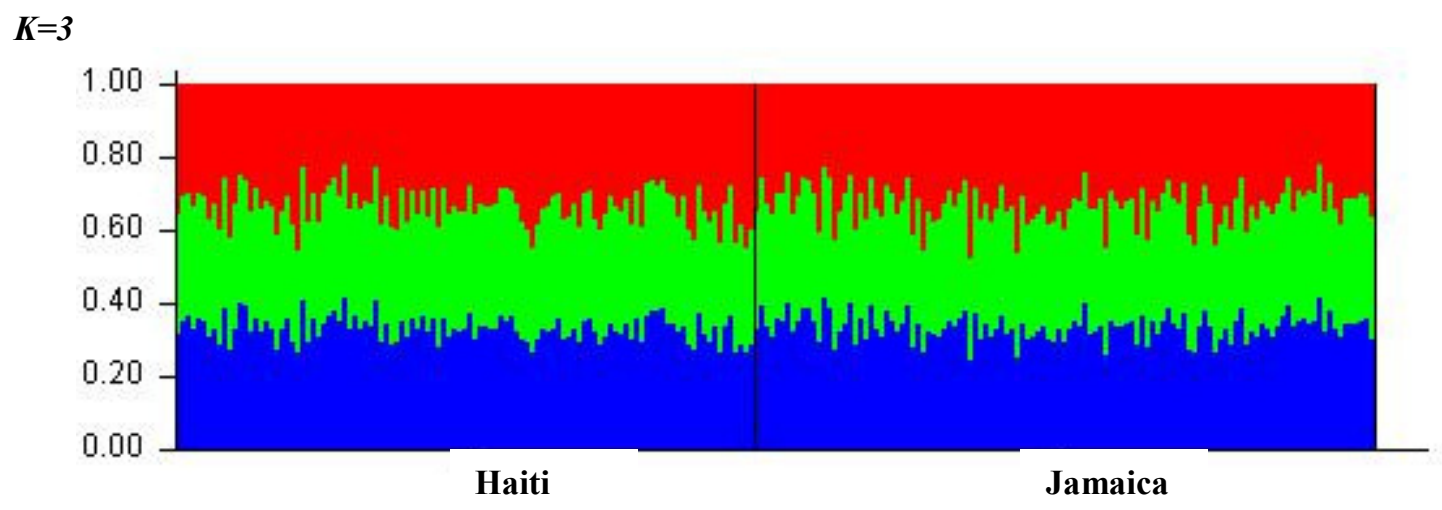

$K=4$

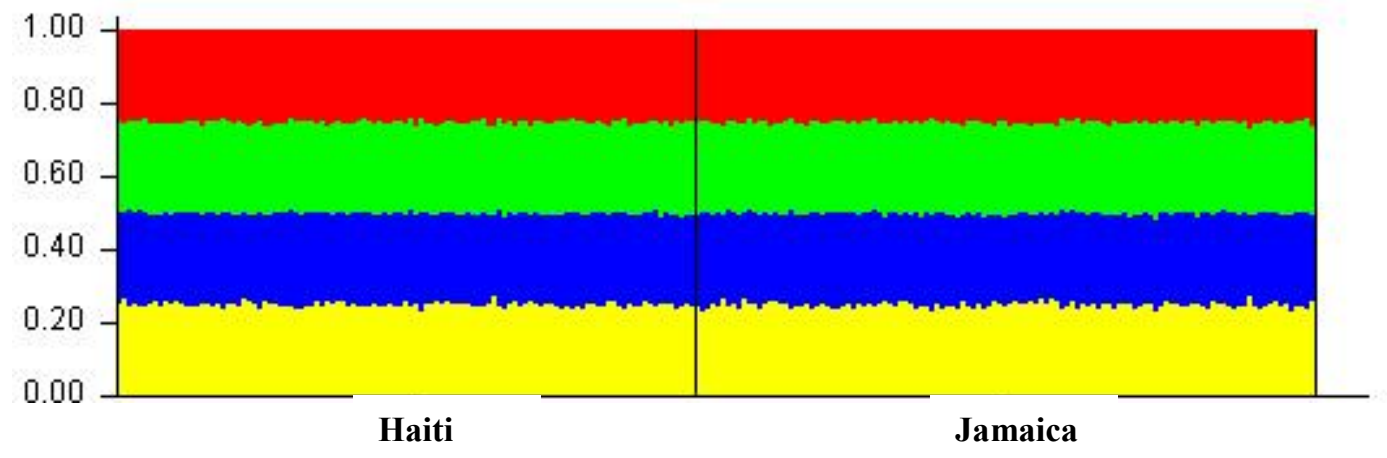


Haiti

\begin{tabular}{lccc} 
Parental Groups & Slow & Fast & All loci \\
\hline African & $0.994 \pm 0.064$ & $0.921 \pm 0.025$ & $0.955 \pm 0.022$ \\
East Asian & $0.006 \pm 0.079$ & $0.000 \pm 0.027$ & $0.003 \pm 0.025$ \\
European & $0.000 \pm 0.067$ & $0.079 \pm 0.032$ & $0.043 \pm 0.027$ \\
\hline
\end{tabular}

Jamaica

\begin{tabular}{lccc} 
Parental Groups & Slow & Fast & All loci \\
\hline African & $0.677 \pm 0.056$ & $0.845 \pm 0.022$ & $0.783 \pm 0.020$ \\
East Asian & $0.038 \pm 0.068$ & $0.081 \pm 0.024$ & $0.057 \pm 0.022$ \\
European & $0.285 \pm 0.058$ & $0.074 \pm 0.029$ & $0.160 \pm 0.024$ \\
\hline
\end{tabular}

New Providence

(Bahamas)

\begin{tabular}{lccc} 
Parental Groups & Slow & Fast & All loci \\
\hline African & $0.809 \pm 0.057$ & $0.762 \pm 0.020$ & $0.780 \pm 0.017$ \\
East Asian & $0.000 \pm 0.069$ & $0.004 \pm 0.021$ & $0.000 \pm 0.019$ \\
European & $0.191 \pm 0.059$ & $0.234 \pm 0.025$ & $0.220 \pm 0.021$ \\
\hline
\end{tabular}

US African American 1

\begin{tabular}{lccc} 
Parental Groups & Slow & Fast & All loci \\
\hline African & $0.793 \pm 0.048$ & $0.766 \pm 0.023$ & $0.780 \pm 0.019$ \\
East Asian & $0.000 \pm 0.059$ & $0.041 \pm 0.024$ & $0.004 \pm 0.022$ \\
European & $0.207 \pm 0.050$ & $0.193 \pm 0.029$ & $0.216 \pm 0.024$ \\
\hline
\end{tabular}

US African American 2

\begin{tabular}{lccc} 
Parental Groups & Slow & Fast & All loci \\
\hline African & $0.774 \pm 0.072$ & $0.791 \pm 0.016$ & $0.782 \pm 0.016$ \\
East Asian & $0.000 \pm 0.088$ & $0.048 \pm 0.017$ & $0.030 \pm 0.018$ \\
European & $0.226 \pm 0.074$ & $0.160 \pm 0.021$ & $0.188 \pm 0.019$ \\
\hline
\end{tabular}

\section{Supporting Information Table 1: Admixture estimates generated for slow vs. fast mutating loci}




\section{DIVERGENT GENETIC STRATA IN FIVEBAHAMIANISLANDS}

\section{A. Introduction}

Depicted as “one of the Atlantic's most geographically [complex] nations” [1], the Bahamas, a chain of 29 islands, 661 cays and 2,387 rocks [2], spans approximately 550 miles from southeastern continental Florida to the northernmost part of Haiti (Cape Haitien). Over the past 500 years, this archipelago has witnessed a series of migratory events emanating from several different sources including, but not limited to, the Arawak Indians, Eleutherian Adventurers, Loyalists, Creole slaves, liberated Africans as well as Chinese, Greek, Jew, Lebanese [3], Jamaican and Haitian [4] migrants. However, the extent that each group contributed to the Bahamian gene pool remains unclear and the degree of genetic admixture between groups is relatively unexplored.

In an early study designed to characterize the genetic composition of the Bahamian population, elevated levels of Hemoglobin $(\mathrm{Hb}) \mathrm{S}, \mathrm{C}$ and $\mathrm{F}$ variants were detected in residents of Bimini and are suggested by the authors to result from the influx of Africans into the island [5]. Genetic ties with continental and/or New World Africans were also observed in investigations performed by Duncan et al. [6] and Herrera et al. [7] utilizing the D1S80 hypervariable marker. More recent reports from my group [8,9] also corroborate the phylogenetic relationships between Bahamians and Africans (from the mainland as well as the New World) detected in the aforementioned studies. Unfortunately, no study to the authors' knowledge has attempted to characterize any of the Bahamian islands (outside of New Providence and Bimini) independently despite the 
high possibility of asymmetrical gene flow from varied sources to each insular population.

Despite the archipelago's minute landmass $\left(13,943 \mathrm{~km}^{2}\right)$ and relatively low population density $\left(22 / \mathrm{km}^{2}\right)[10]$, the Bahamas exhibits some of the highest murder, assault and rape rates per capita [11], reinforcing the importance of Bahamian-specific DNA databanks. Although STR datasets are available for the general (All) Bahamian population [12] as well as for a collection from New Providence [8], differences observed between the two may signal statistically significant heterogeneity among the islands of the archipelago. Therefore, the current investigation was undertaken to establish anthropologically well-characterized datasets across the 15 Identifiler forensic loci for several of the more densely populated islands in the Bahamian chain, namely Abaco, Eleuthera, Exuma, Grand Bahama and Long Island. Also, the genetic profiles of the five Bahamian islands under scrutiny were employed to assess their phylogenetic relationships to pertinent geographically targeted reference collections.

\section{B. Materials and Methods}

\section{Sample Collection and DNA Isolation}

Buccal swabs were collected from 535 unrelated individuals residing in the islands of Abaco $(\mathrm{n}=111)$, Eleuthera $(\mathrm{n}=112)$, Exuma $(\mathrm{n}=92)$, Grand Bahama $(\mathrm{n}=133)$ and Long Island (n=87) (Fig. 1) with informed consent. Genealogical information for every donor was recorded for at least two generations to establish local descent. All samples were procured according to the ethical guidelines stipulated by the institutions 
involved. Genomic DNA was extracted using the Gentra Buccal Cell Kit (Puregene, Gentra Systems, Minneapolis, MN) according to the manufacturers' instructions and stored at $-80^{\circ} \mathrm{C}$ in the Puregene ${ }^{\mathrm{TM}}$ DNA hydration solution (10mM Tris, $1 \mathrm{mM}$ EDTA, $\mathrm{pH}$ 8.0). DNA quantification was performed with a NanoDrop 2000 spectrophotometer (NanoDrop products, Wilmington, DE) and each sample was diluted to a final concentration of $0.75 \mathrm{ng} / \mu \mathrm{l}$ prior to PCR amplification.

\section{STR Genotyping}

All samples were amplified across the 15 autosomal STR loci (D8S1179, D21S11, D7S820, CSF1PO, D3S1358, TH01, D13S317, D16S539, D2S1338, D19S433, vWA, TPOX, D18S51, D5S818 and FGA) included in the commercially available AmpFlSTR ${ }^{\circledR}$ Identifiler kit (Applied Biosystems, Foster City, CA). Amplification reactions were performed in an Eppendorf Mastercycler ${ }^{\circledR}$ gradient (Eppendorf, Hamburg, Germany) according to the manufacturer's recommended protocols [13]. Following amplification, PCR products were separated by multicapillary electrophoresis in an ABI

3130xl Genetic Analyzer using POP $7^{\mathrm{TM}}$ as the separation medium and ABI GeneScan ${ }^{\mathrm{TM}}$ $500 \mathrm{LIZ}^{\circledR}$ as the internal size standard. The resulting STR fragments were genotyped with the GeneMapper ${ }^{\circledR}$ software v3.2, which determines allele size and designation through comparisons to the internal size standard and allelic ladder, respectively. Off-ladder alleles are those characterized by incomplete repeat sequences [14]. 


\section{Statistical and phylogenetic analyses}

Allelic frequencies for the 15 STR loci typed in the collections from Abaco, Eleuthera, Exuma, Grand Bahama and Long Island were determined with the GenePop v 3.4 program [15]. To compare the distribution of alleles among these populations, histograms for each STR locus were constructed with Microsoft Excel. The Arlequin version 3.11 software package [16] was utilized to compute observed and expected heterozygosities $\left(\mathrm{H}_{\mathrm{o}}\right.$ and $\mathrm{H}_{\mathrm{e}}$, respectively) as well as gene diversity indices (GDI) and Hardy-Weinberg equilibrium (HWE) $P$ values. Possible inbreeding in these island populations was assessed through calculation of $\mathrm{F}_{\text {IS }}$ values for each of the $15 \mathrm{STR}$ markers with GenePop v 3.4 [15]. Additionally, several parameters of forensic and population genetics interest, including matching probability (MP), power of discrimination (PD), polymorphic information content (PIC), power of exclusion (PE) and typical paternity index (TPI) were estimated employing the PowerStats 1.2 software $[17,18]$.

Genetic distances $\left(F_{s t}\right)$ among the five Bahamian populations studied and the 43 geographically targeted reference collections $[8,9,19-53]$ were computed using the GeneDist option of the PHYLIP 3.52c software [54]. Utilizing the $F_{s t}$ distances generated, multidimensional scaling (MDS) plots were constructed with the Statistical Package for the Social Sciences (SPSS) version 14.0 [55]. The analysis was performed at the all populations level and was repeated to include only the African and Africanderived (Bahamian, Afro-American and Afro-Caribbean) populations to better assess phylogenetic associations between them. Because of its sizeable Austronesian genetic component [27], Madagascar was excluded from the latter analysis. 
To determine the African, East Asian, European and Native American genetic contributions made to the gene pools of the Bahamian populations under study, admixture determinations employing the Weighted Least Square (WLS) method described in Long et al. [56] were performed using SPSS version 14.0 [55]. In addition to quantifying admixture proportions for Abaco, Eleuthera, Exuma, Grand Bahama and Long Island, the impact of parental populations on New Providence was reevaluated to assess potential influences from the Native American collections, which were not included in my earlier studies $[8,9]$

Inter-, intra- and total population $\left(\mathrm{G}_{\mathrm{st}}, \mathrm{H}_{\mathrm{s}}\right.$ and $\mathrm{H}_{\mathrm{t}}$, respectively) genetic variance values were calculated with the DISPAN program [57]. The collections examined were assembled into population groups representing the different biogeographic areas demarcated in Table 1. In addition, G-tests [58] were conducted to determine the statistical significance of genetic differences among all 48 populations examined. The significance of each pair-wise comparison was assessed through 100,000 simulations, both before (in bold) and after (in italics) application of a conservative Bonferroni adjustment $(\alpha=0.05 / 1128=0.000044326)$.

Population substructure was ascertained using the Structure software v. 2.3.3 [59] available at http://pritch.bsd.uchicago.edu/structure/html. For this test, the autosomal profiles of individuals residing in Abaco, Eleuthera, Exuma, Grand Bahama, Long Island and New Providence were analyzed alongside the reference African (Kenya, Madagascar, Rwanda Hutu and Tutsi), East Asian (Atayal and Ami from Formosa, and Java [60]), Native American (Kakchikel, K'iche and Yucatan Mayan populations) and European (Spain, Sweden and Greece) populations for which genotypic data has been provided by 
the authors. Prior to analysis, incomplete alleles were replaced with integers (outside of the normal allele range) and missing and/or null alleles were assigned a value of -9 as indicated by the Structure program. Clustering was performed at $K=3$ and $K=4$ using the admixture model that does not require population of origin information and assumes allele frequencies in the populations under study to be correlated to each other. For each $K$ value, a burn-in period of 20,000 generations followed by 20,000 Markov chain Monte Carlo (MCMC) simulations was employed.

\section{Results}

Intra-population Diversity

Allelic frequencies generated for the collections from Abaco, Eleuthera, Exuma, Grand Bahama and Long Island are listed in Supplementary Tables 1 through 5, respectively. Also provided in Supplementary Table 6 are several indices of forensics and population genetics interest. Examination of allelic distributions among these populations reveals no substantial differentiation in allelic counts, which range from 144 in Exuma to 154 in Abaco (Table 2), but does illustrate that each collection is characterized by the presence of unique alleles in relation to the other Bahamian islands surveyed (Supplementary Figure 1); Abaco was found to possess the greatest number of distinct alleles (12) while both Grand Bahama and Long Island exhibit the least (3). These findings are not surprising given that Abaco harbors the highest observed average heterozygosity value (0.80187) and Long Island, the lowest (0.78084) (Table 2). Interestingly, each Bahamian population possesses seven or more loci with lower than 
expected $\mathrm{H}_{\mathrm{o}}$ values, yet departures from HWE expectations (Table 3 ) were detected only in the collections from Abaco (D2S1338), Exuma (D8S1179) and Long Island (D8S1119, D19S433 and FGA). These deviations, however, did not persist after application of the Bonferroni correction $(\alpha=0.05 / 15=0.0033)$.

The highest GDI values are noted for D2S1338 in Abaco and Grand Bahama, D18S51 in Eleuthera, and FGA in both Long Island and Exuma. The D13S317 locus, in contrast, exhibits the lowest genetic variability in all populations examined, with the exception of Long Island. Of the 15 STR markers, D2S1338 (Abaco, Eleuthera and Grand Bahama), D18S51 (Abaco and Long Island) and FGA (Exuma) possess the strongest discriminative power while D5S818 (Long Island), D3S1358 (Abaco) and D13S317 (Eleuthera, Exuma and Grand Bahama) were found to be the least discriminatory (Supplementary Table 6). Although the combined power of exclusion (CPE) is $>99.9 \%$ for all five collections, the combined power of discrimination (CPD) values differ, ranging from $67.3 \%$ for Abaco to $73.0 \%$ for Exuma (Table 2).

The 15 Identifiler loci in the Bahamian (0.80162) and New World African (0.79785) assemblages exhibit the highest average intra-population variance values of all groups examined, including the continental African conglomerate (0.79404), while the lowest overall $\mathrm{H}_{\mathrm{s}}$ levels were observed among the Native American (0.72701) and Asian (0.78131) groups of populations (Table 4). Even though the Bahamian group displays the highest intra-population diversity, three islands, Exuma, Long Island and Grand Bahama, were found to possess either one or two loci with statistically significant heterozygote deficiencies (Table 3). 


\section{Inter-population Diversity}

The G-test results (Supplementary Table 7) reveal statistically insignificant genetic differences between the Bahamian collections and several of the continental African populations (i.e., Angola, Equatorial Guinea, Guinea-Bissau and the Bantu group from Kenya) either before or after application of the Bonferroni correction. In addition, Abaco, Eleuthera, Exuma, Grand Bahama and New Providence were not found to differ significantly from the other New World groups of African descent (Haiti, Jamaica and the two US African American collections), likely attributed to genetic contributions from similar ancestral sources and/or gene flow among these populations. Interestingly, Long Island was the only Bahamian collection to exhibit genetic heterogeneity with all 47 populations examined in the pair-wise analyses.

In the global MDS plot (Fig. 2A), five clusters are distinctly delineated according to biogeographic origin. In the upper left quadrant, a tightly packed European group lies proximal to the more loosely associated conglomerate of East Asian populations while the Native American collections occupy the extreme lower left portion of the graph. The Latin American groups form an assemblage at the center of the projection with Brazilians and Puerto Rican Americans occupying the right half of the plot close to the African collections. Within the African cluster, a clear demarcation between the New World (with the exemption of Haiti) and continental Africans (excluding Angola, Madagascar and Mozambique) is apparent; Abaco, Eleuthera, Exuma, Grand Bahama, Long Island, New Providence, Jamaica and the two US Afro-American collections (US1 and US2) segregate together with Angola, Mozambique and Madagascar at the periphery of the continental African conglomerate, occupying a transitional position between this group of 
populations and the Latin American and European clusters. Specifically, within the New World/Angola/Madagascar/Mozambique assemblage, Abaco partitions centrally with Jamaica, New Providence, Angola and the two US African American populations while Eleuthera, Grand Bahama, Exuma and Long Island plot peripherally. Also noteworthy is Long Island's position nearest to Madagascar, an island off the East African coast, which shares approximately $33.9 \%$ of its autosomal component with Austronesians [27].

Phylogenetic relationships among the Bahamian, Afro-Caribbean, Afro-American and continental African populations apparent in the global projection are also noted in the MDS analysis employing only the African and African-derived groups (Fig 2B). In both graphs, Abaco partitions along with New Providence and the two US African American (US1 and US2) collections. In Figure 2B, however, the above mentioned New World populations cluster not only with Angola but also with Equatorial Guinea and Nigeria, two additional, historically known West African sources of slaves. Genetic ties between Bahamians and West Africans are also evident in the segregation of Exuma with GuineaBissau and Grand Bahama with the Bantu group from Kenya as well as in the positioning of Eleuthera intermediate to the collections from Angola and Guinea-Bissau. Long Island, in contrast, plots as an outlier, displaying a considerable degree of genetic separation from the other Bahamian populations as well as the continental and New World African collections.

When $\mathrm{G}_{\mathrm{st}}$ values (Table 4) are compared, the Bahamian populations exhibit one of the lowest average inter-population diversity levels (0.00583) while the Native American group possesses the highest $(0.02412)$. The Bahamian assemblage was also found to 
exhibit the highest total population $\left(\mathrm{H}_{\mathrm{t}}\right)$ variance $(0.80633)$, followed by the continental (0.80287) and New World (0.80053) African conglomerates.

Results of the admixture analyses (Table 5) indicate that the largest genetic contributor to the Bahamian gene pool is continental Africa, with Long Island, Abaco, Eleuthera, Grand Bahama and Exuma sharing approximately $65.4 \%, 75.0 \%, 80.3 \%$, $86.7 \%$ and $90.4 \%$ of their autosomal component with the African collections, respectively. Influences from the European populations are the second largest for all hybrid collections but are most prominent in Long Island (26.9\%). East Asian genetic signals were only present in Long Island (7.7\%), Abaco (1.5\%) and Grand Bahama $(0.5 \%)$ while Native American influences were detected in Grand Bahama (3.9\%), Exuma (5.9\%) and Eleuthera (6.0\%). Recalculation of admixture estimates for New Providence identifies proportions similar to those observed in my earlier reports $[8,9]$ with only minimal $(0.06 \%)$ genetic contributions from Native Americans.

In the Structure bar plots (Supplementary Figure 2), assessment of the African (red and yellow colors at $K=3$ and $K=4$, respectively) and European (blue and red colors at $K=3$ and $K=4$, respectively) ancestral components in the Bahamian populations was possible at $K=3$ and $K=4$. At both $\mathrm{K}$ values, the Bahamian groups exhibit patterns mirroring those of the continental African collections, although Long Island, Abaco, Eleuthera and New Providence also possess distinct groups of individuals whose profile is consistent with the one observed in the European populations. Membership proportions (Table 6) indicate that the majority of samples in each Bahamian collection share membership within the African cluster, with the highest percentages being observed in Exuma ( $80.1 \%$ for $K=3$ and $75.3 \%$ for $K=4)$ and the lowest in Long Island $(59.4 \%$ for 
$K=3$ and $53.2 \%$ for $K=4$ ). European membership proportions, which range from $11.5 \%$ to $28.5 \%$ when $K=3$ and from $9.3 \%$ to $20.6 \%$ when $K=4$, are comparable to those obtained from this parental group in the admixture tests (Table 5), with Long Island receiving the strongest European genetic signals and Exuma the weakest. Native American proportions (with the exception of New Providence, which exhibits larger genetic contributions from Native Americans) generated by both the SPSS (uses Long's WLS approach) and Structure programs are commensurate yet, the percentage of East Asian DNA in each population's gene pool is substantially higher in the membership proportion results (Table 6). The relatively stronger genetic signals from the East Asian group are likely the result of their shared genetic profiles with the European collections.

\section{Discussion}

In the present study, allelic frequencies for the 15 Identifiler loci routinely employed in forensic applications are reported for five Bahamian collections representing the more populous northwest (Grand Bahama and Abaco) and central (Eleuthera, Exuma and Long Island) portions of the archipelago. Although several published reports [5-9] have established genetic links between Bahamians and Africans, the current investigation represents the first to delineate phylogenetic relationships and identify differential genetic stratification among the Bahamian islands.

As a group, the Bahamian populations exhibit an average of 152 allelic types, however, when considered on an individual basis, total allele numbers vary, ranging from 144 in Exuma (Table 2) to 173 in New Providence [8]. This average, although comparable to that of the continental African collections (151), is lower than the allelic 
counts for the other New World groups of African descent [Afro-Caribbean (159) and Afro-American (165)]. The reduced number of alleles present in each of the Bahama Island populations (with the exception of New Providence), in combination with the seven or more loci that display lower than expected $\mathrm{H}_{\mathrm{o}}$ values, is likely the result of genetic isolation, bottlenecks and/or inbreeding. Another plausible explanation is that the Bahamas has not witnessed the degree of genetic admixture experienced by other nations within the Caribbean basin $[61,62]$. Despite expectations of decreased genetic variability, the Bahamian islands examined actually exhibit the highest intra-population variance $\left(\mathrm{H}_{\mathrm{s}}\right)$ of all groups examined (Table 4), suggesting that each population's gene pool represents a mosaic of several different groups. This high level of diversity is supported by the Structure analyses which reflect several degrees of sub-population structure in the populations of the five Bahamian islands. Furthermore, Abaco (0.81441), Grand Bahama $(0.80187)$ and Eleuthera (0.79986) possess average heterozygosities that exceed the mean values for the mainland African collections (0.79777) as well as both New World African groups [Afro-Caribbean (0.78974) and Afro-American (0.79977)], reflecting a high level of diversity throughout the island chain.

When inter-population diversity (Table 4) is examined, Bahamians possess the third to lowest value (0.00583) after New World Africans (0.00334) and Europeans (0.00337). The higher average $\mathrm{G}_{\mathrm{st}}$ in the populations from the Bahamas, as compared to that among the New World African collections, may result from genetic drift, geographical isolation and/or differential gene flow. Genetic stratification among the islands is also evident in the widespread segregation of the Bahamian collections in both MDS projections (Fig. 2A,B) as well as in the sub-population structure patterns observed 
in the Structure bar graphs (Supplementary Figure 2). Of particular interest is the Long Island population, which displays a considerable degree of heterogeneity from the other Bahamian collections, not only in the aforementioned analyses but also in the G-test (Supplementary Table 7) where it exhibits statistically significant genetic differences with all populations in the pair-wise comparisons, even after application of the Bonferroni adjustment.

Admixture (Table 5) as well as membership proportions (Table 6) for Abaco, Eleuthera, Exuma, Grand Bahama, Long Island and New Providence identify continental Africa as the primary genetic contributor to Bahamian gene pool. These findings are anticipated given the large number of Creole slaves and liberated Africans transported to the colony during the slave trade [63] and post-slave trade [2] eras, respectively. In addition, migrants from several other African-derived populations in the West Indies, including Turks and Caicos, Haiti, Jamaica and Barbados, are known to have been incorporated into Bahamian society $[4,64]$. The high percentage of African DNA present is also reflected in the Structure bar plots (Supplementary Figure 2), where each Bahama Island population was found to share membership in the same cluster of similar profiles with the continental Africans collections, likely inferring a common genetic ancestry [65]. Furthermore, several alleles representative of the continental and New World Africans collections examined in the current report are observed in Abaco (D21S11-24.3 and FGA-30.2), Exuma (D21S11-33.1, D21S11-37 (also present in Mexico [42]), D7S820-6 (also detected in Honduras [45]) and vWA-12), and Long Island (FGA-31.2 (also exhibited by Caracas [41])). 
Although each hybrid shares the majority of its autosomal component with the African collections, substantial variation in admixture (Table 5) as well as membership proportions (Table 6) exists among the islands. The relatively lower percent contribution (65.4\% in the admixture tests; $59.4 \%(K=3)$ and $53.2 \%(K=4)$ in the membership proportion results) detected in Long Island is supported by the segregation of this population away from the New World and continental African collections (excluding Madagascar) in both MDS projections (Fig. 2A,B). Long Island also exhibits significant heterogeneity with all populations in the G-test (Supplementary Table 7). Meanwhile, the positioning of Abaco, New Providence and Eleuthera near the center of Figure 2B corroborates the intermediate level of African genetic input (Tables 5 and 6) detected in these islands while Grand Bahama and Exuma, which receive larger African contributions (Tables 5 and 6), lie on the periphery of the graph proximal to GuineaBissau and Namibia, respectively. Additionally, the Bahamian populations (with the exception of Long Island) were not found to differ significantly from Angola, GuineaBissau, Equatorial Guinea and/or Kenya in the G-test pair-wise analyses.

A contrasting distribution pattern of European genetic influences is noted throughout the archipelago, with Long Island displaying the strongest genetic signal and Exuma, the weakest (Tables 5 and 6). Genetic input from Europeans stems from two main sources: (1) British Independents from Bermuda [66] and (2) the Loyalists. Sharer [2], in his work on Bahamian population growth, reports "a peculiar distribution of Europeans" in the Bahamas, noting that those islands permanently settled by the Bermudian colonists (i.e., Eleuthera, New Providence and Abaco) possessed the highest proportions of Europeans in 1943, a trend that is observed not only in the admixture 
analyses (Table 5) but also in the bar graphs (Supplementary Figure 2) and membership proportions (Table 6) generated by Structure. Additional sources of European gene flow include Greeks, Eastern European Jews [3], Canadians, Americans and migrants from the United Kingdom [64].

Genetic signals from the East Asian populations are restricted to the collections from Long Island (7.7\%), Abaco (1.5\%) and Grand Bahama (0.05\%) in the admixture tests (Table 5) but are detected in all six populations (ranging from $10.1 \%$ to $21.4 \%$ at $K=4$ ) in the membership proportion results (Table 6), with the most notable contribution in both analyses being observed in Long Island. Genetic influences from East Asia are most likely attributed to the two waves of Chinese immigration that took place during the late nineteenth (1879) and early twentieth (1920s) centuries [67]. Unfortunately, records regarding the number of Chinese migrants entering the colony and the island(s) on which they settled are scanty. Native American contributions, on the other hand, are detected in New Providence (0.6\%), Grand Bahama (3.9\%), Exuma (5.9\%), and Eleuthera (6.0\%) in the admixture analyses (Table 5) and in all six collections (ranging from $4.4 \%$ to $13.8 \%$ at $K=4$ ) in the membership proportion results (Table 6). Genetic input from this group, although minimal, may represent genetic signatures of the indigenous Arawak Indian population. A more plausible explanation is through immigration from Turks and Caicos, a group of islands to the southeast of the Bahamas, which over the years has received a number of migrants from the Dominican Republic [68]. According to Torroni et al. [69], the Dominican maternal gene pool is characterized by an Amerindian-specific component. 
Considering the genetic differences among the five Bahamian islands examined in this study, it is not surprising to uncover dissimilarities among the previously published All Bahamian dataset [12], the database for New Providence [8] and the insular-specific datasets generated. These differences include the absence of frequency data for loci D2S1338 and D19S433 which were not typed for the general Bahamian population. The incorporation of these two additional hypervariable markers provides greater discriminatory resolution to this battery of autosomal STR markers. Also, in the All Bahamian dataset small and large alleles are grouped together (e.g., alleles 8, 9, 10 and 11 are designated as $<11$ at D18S51) at several loci. The clustering of the smaller and/or larger alleles (e.g., $<11$ at D18S51 and $<18$ and $>30$ at FGA) prevents assessment of the specific allele in question when utilizing this database for forensic statistical calculations. Furthermore, an 11 year old database, considering the population dynamics (e.g., recent migrations from neighboring countries, including Haiti and Jamaica) of the islands, may not accurately represent present-day allelic frequencies.

\section{E. Conclusion}

Overall, my data reflects a high level of diversity throughout the Bahamian chain indicating that Bahamians are a heterogeneous group, receiving genetic contributions from a wide array of sources. Although all five populations surveyed display a closer genetic relationship with Africans than with any other group examined, differential genetic stratification among them is noted in the MDS and admixture analyses as well as in the membership proportion results. In addition, the Structure bar plots reveal collagelike profiles signaling the presence of sub-population structure in the islands of Abaco, 
Eleuthera, Long Island and New Providence. These genetic differences, in combination with the presence of alleles not in common among the five Bahamian islands, indicate island-specific heterogeneity within the archipelago. This is especially the case when considering the Long Island population, where the G-test indicates statistically significant genetic differences in all pair-wise analyses (both before and after application of the Bonferroni correction) including those with the other four Bahamian populations studied as well with the New Providence and New World African (Afro-American and AfroCaribbean) collections. 


\section{REFERENCES}

[1] D. Porter, D. Prince, Frommer's Bahamas 2010, Willey Publishing Inc., New Jersey, 2009.

[2] C.J. Sharer, The population growth of the Bahama Islands, Dissertation, University of Michigan, 1955.

[3] G. Saunders, The Bahamas: a family of islands, Macmillan Education, London, 2000.

[4] K.L. Tinker, Perspectives on West Indian migration to the Bahamas: pre-Columbian to Bahamian independence in 1973, Dissertation, Florida State University, 1998.

[5] R.A. Halberstein, J.E. Davies, K. Mack, Hemoglobin variations on a small Bahamian island, Am J Phy Anthropol 55 (1981) 217-221.

[6] G. Duncan, E. Thomas, J.C. Gallo, L.S. Baird, J. Garrison, R.J. Herrera, Human phylogenetic relationships according to the D1S80 locus, Genetica 98 (1996) 277-287.

[7] R.J. Herrera, L.R. Adrien, L.M. Ruiz, N.Y. Sanabria, G. Duncan, D1S80 single-locus discrimination among African populations, Hum Biol 76 (2004) 87-108.

[8] T.M. Simms, C. Garcia, S. Mirabal, Q. McCartney, R.J. Herrera, The genetic legacy of the Transatlantic Slave Trade in the island of New Providence, Forensic Sci Int: Genet 2 (2008) 310-317.

[9] T.M. Simms, C.E. Rodriguez, R. Rodriguez, R.J. Herrera, The genetic structure of populations from Haiti and Jamaica reflect divergent demographic histories, Am J Phys Anthropol 142 (2010) 49-66.

[10] Department of Statistics of the Bahamas, The 2000 census of population and housing tables (All Bahamas). http://statistics.bahamas.gov.bs/archives.php?cat=70. Accessed 06 August 2010.

[11] United Nations Office on Drugs and Crime, Crime, violence, and development: trends, costs, and policy options in the Caribbean, Report No. 37820, 2007.

[12] B. Budowle, T. Moretti, A.L. Baumstark, D.A. Defenbaugh, K.M. Keys. Population data on the thirteen CODIS core short tandem repeat loci in African Americans, U.S. Caucasians, Hispanics, Bahamians, Jamaicans, and Trinidadians, J Forensic Sci 44 (1999) 1277-1286.

[13] Applied Biosystems, AmpFlSTR Identifiler PCR amplification kit user's manual. Applied Biosystems, California, 2001. 
[14] J.M. Butler, Forensic DNA typing, biology, technology, and genetics of STR markers, second ed, Elsevier Academic Press, Massachusetts, 2005.

[15] M. Raymond, F. Rousset, GENEPOP (Ver. 1.2): a population genetics software for exact test and ecumenicism, J Hered 86 (1995) 248-249.

[16] L. Excoffier, G. Laval, S. Schneider, Arlequin ver 3.0: an integrative software package for population genetics data analysis, Evol Bioinform Online 1 (2005) 47-50.

[17] C.H. Brenner, J.W. Morris, Paternity index calculation in single locus hypervariable DNA probes: validation and other studies, in: Proceedings for the international symposium of human identification, Promega Corporation, Wisconsin, 1990, pp 21-55.

[18] A. Tereba, Tools for analysis of population statistics, in: Profiles in DNA, v.2, Promega Corporation, Wisconsin, 1999, pp 14-16.

[19] A.M. Gross, B. Budowle, Minnesota population data on 15 STR loci using the Identifiler ${ }^{\circledR}$ kit. J Forensic Sci 51 (2006) 1410-1413.

[20] J.M. Butler, R. Schoske, P.M. Vallone, J.W. Redman, M.C. Kline, Allele frequencies for 15 autosomal STR loci on U.S. Caucasian, African American, and Hispanic populations, J Forensic Sci 48 (2003) 908-911.

[21] S. Beleza, C. Alves, F. Reis, A. Amorim, A. Carracedo, L. Gusmão, 17 STR data (AmpFISTR Identifiler and Powerplex 16 system) from Cabinda (Angola), Forensic Sci Int 141 (2004) 193-196.

[22] J.V. Pereira, A. Serra, M.J. Anjos, M.C. Vide, F. Corte-Real, D.N. Vieira, Allele frequencies for fifteen STR loci in Guinea-Bissau population, J Forensic Sci 50 (2005) 243-244.

[23] C. Alves, L. Gusmão, A.M. López-Parra, M. Soledad Mesa, A. Amorim, E. ArroyoPardo, STR allelic frequencies for an African population sample (Equatorial Guinea) using AmpFISTR Identifiler and Powerplex 16 kits, Forensic Sci Int 148 (2005) 239-242.

[24] T. Muro, J. Fujihara, S. Imamura, H. Nakamura, T. Yasuda, H. Takeshita, Allele frequencies for 15 STR loci in Ovambo population using AmpFISTR ${ }^{\circledR}$ Identifiler kit. Leg Med 10 (2008) 157-159.

[25] C. Hohoff, M. Schürenkamp, B. Brinkmann, Meiosis study in a population sample from Nigeria: allele frequencies and mutation rates of 16 STR loci, Int J Legal Med 123 (2009) 259-261.

[26] E.M. Shepard, R.J. Herrera, Genetic encapsulation among Near Eastern populations, J Hum Genet 51 (2006) 467-476. 
[27] M. Regueiro, S. Mirabal, H. Lacau, J.L. Caeiro, R.L. Garcia-Bertrand, R.J. Herrera, Austronesian genetic signature in East African Madagascar and Polynesia, J Hum Genet 53 (2008) 106-120.

[28] C. Alves, L. Gusmão, A. Damasceno, B. Soares, A. Amorim, Contribution for an African autosomic STR database (AmpFISTR Identifiler and Powerplex 16 system) and a report on genotypic variations, Forensic Sci Int 139 (2004) 201-205.

[29] B.W. Forward, M.W. Eastman, T.B. Nyambo, R.E. Ballard, AmpFiSTR ${ }^{\circledR}$ Identifiler ${ }^{\mathrm{TM}}$ STR allele frequencies in Tanzania, Africa, J Forensic Sci 53 (2008) 245247.

[30] M. Regueiro, J.C. Carril, M.L. Pontes, M.F. Pinheiro, J.R. Luis, B. Caeiro, Allele distribution of 15 PCR-based loci in the Rwanda Tutsi population by multiplex amplification and capillary electrophoresis, Forensic Sci Int 143 (2004) 61-63.

[31] L. Gusmão, P. Sánchez-Diz, I. Gomes, C. Alves, Á. Carracedo, M.J. Prata, A. Amorim, Genetic analysis of autosomal and Y-specific STRs in the Karimojong population from Uganda, Int Congr Ser 1288 (2006) 213-215.

[32] A. Kido, Y. Dobashi, N. Fujitani, M. Hara, R. Susukida, H. Kimura, M. Oya, Population data on the AmpFlSTR Identifilier loci in Africans and Europeans from South Africa, Forensic Sci Int 168 (2007) 232-235.

[33] S.P. Hu, X.J. Yu, J.W. Liu, K.L. Cai, Analysis of STR polymorphisms in the Choa Shan population in South China, Forensic Sci Int 147 (2005) 93-95.

[34] M. Hashiyada, Y. Itakura, T. Nagashima, M. Nata, M. Funayama, Polymorphism of 17 STRs by multiplex analysis in Japanese population, Forensic Sci Int 133 (2003) 250253.

[35] Y. Kim, J. Hwang, Y. Kim, S. Lee, N. Chung, H. Goh, C. Kim, D. Kim, Allele frequencies of 15 STR loci using AmpFlSTR Identifiler kit in a Korean population, Forensic Sci Int 136 (2003) 92-95.

[36] L.H. Seah, N.H. Jeevan, M.I. Othman, P. Jaya, Y.S. Ooi, P.C. Wong, S.S. Kee, STR data for the AmpFlSTR Identifiler loci in three ethnic groups (Malay, Chinese, Indian) of the Malaysian population, Forensic Sci Int 138 (2003) 134-137.

[37] M.C.A. De Ungria, R.K. Roby, K.A. Tabbada, S. Rao-Coticone, M.M.M. Tan, K.N. Hernandez, Allele frequencies of 19 STR loci in a Philippine population generated using AmpFISTR multiplex and ALF singleplex systems, Forensic Sci Int 152 (2005) 281-284. 
[38] Z. Wang, R. Yu, F. Wang, X. Li, T. Jin, Genetic polymorphisms of 15 STR loci in Han population from Shaanxi (NW China), Forensic Sci Int 147 (2005) 89-91.

[39] F.B. Kohlrausch, S.M. Callegari-Jacques, L.T. Tsuneto, M.L. Petzl-Erler, K. Hill, A.M. Hurtado, F.M. Salzano, M.H. Hutz, Geography influences microsatellite polymorphism diversity in Amerindians, Am J Phys Anthropol 126 (2005) 463-470.

[40] L. Ibarra-Rivera, S. Mirabal, M.M. Regueiro, R.J. Herrera, Delineating genetic relationships among the Maya, Am J Phys Anthropol 135 (2008) 329-347.

[41] M.A. Chiurillo, A. Morales, A.M. Mendes, N. Lander, F. Tovar, A. Fuentes, J.L. Ramírez, Genetic profiling of a central Venezuelan population using 15 STR markers that may be of forensic importance, Forensic Sci Int 136 (2003) 99-101.

[42] S. Hernández-Gutiérrez, P. Hernández-Franco, S. Martínez-Tripp, M. Ramos-Kuri, H. Rangel-Villalobos, STR data for 15 loci in a population sample from the central region of Mexico, Forensic Sci Int 151 (2005) 97-100.

[43] A. Rodríguez, G. Arrieta, I. Sanóu, M.C. Vargas, O. García, I. Yurrebaso, J.A. Pérez, M. Villalta, M. Espinoza, Population genetic data for 18 STR loci in Costa Rica, Forensic Sci Int 168 (2007) 85-88.

[44] J.A. Morales, J.C. Monterrosa, J. Puente, Population genetic data from El Salvador (Central America) using AmpFlSTR ${ }^{\circledR}$ Identifiler ${ }^{\mathbb{R}}$ PCR Amplification Kit, Int Congr Ser 1261 (2004) 223-225.

[45] M. Matamoros, Y. Pinto, F.J. Inda, O. García, Population genetic data for 15 STR loci (Identifiler ${ }^{\mathrm{TM}}$ kit) in Honduras, Leg Med 10 (2008) 281-283.

[46] L.P. Bernal, L. Borjas, W. Zabala, M.G. Portillo, E. Fernández, W. Delgado, F. Tovar, N. Lander, M.A. Chiurillo, J.L. Ramírez, O. García, Genetic variation of 15 STR autosomal loci in the Maracaibo population from Venezuela, Forensic Sci Int 161 (2006) 60-63.

[47] A. Gorostiza, A. González-Martín, C.L. Ramírez, C. Sánchez, C. Barrot, M. Ortega, E. Huguet, J. Corbella, M. Gené, Allele frequencies of the 15 AmpFISTR Identifiler loci in the population of Metztitlán (Estado de Hidalgo), México, Forensic Sci Int 166 (2007) $230-232$.

[48] J. Zúñiga, M. Ilzarbe, V. Acunha-Alonzo, F. Rosetti, Z. Herbet, V. Romero, I. Almeciga, O. Clavijo, J.N.H. Stern, J. Granados, M. Fridkis-Hareli, P. Morrison, J. Azocar, E.J. Yunis, Allele frequencies for 15 autosomal STR loci and admixture estimates in Puerto Rican Americans, Forensic Sci Int 164 (2006) 266-270. 
[49] A.C. de Souza Góes, D.A. da Silva, E.H. Fonseca Gil, M.T.D. da Silva, R.W. Pereira, E.F. de Carvalho, Allele frequencies data and statistic parameters for 16 STR loci - D19S433, D2S1338, CSF1PO, D16S539, D7S820, D21S11, D18S51, D13S317, D5S818, FGA, Penta E, TH01, vWA, D8S1179, TPOX, D3S1358 - in the Rio de Janeiro population, Brazil, Forensic Sci Int 140 (2004) 131-132.

[50] P. Sánchez-Diz, P.G. Menounos, A. Carracedo, I. Skitsa, 16 STR data of a Greek population, Forensic Sci Int: Genet 2 (2008) e71-e72.

[51] V. Lopes, A. Serra, J. Gamero, L. Sampaio, F. Balsa, C. Oliveira, L. Batista, F. Corte-Real, D.N. Vieira, M.C. Vide, M.J. Anjos, M. Carvalho, Allelic frequency distribution of 17 STRs from Identifiler and PowerPlex-16 in Central Portugal area and the Azores archipelago, Forensic Sci Int: Genet 4 (2009) e1-e7.

[52] M.V. Camacho, C. Benito, A.M. Figueiras, Allelic frequencies of the 15 STR loci included in the AmpFISTR ${ }^{\circledR}$ Identifiler ${ }^{\mathrm{TM}}$ PCR Amplification kit in an autochthonous sample from Spain, Forensic Sci Int 173 (2007) 241-245.

[53] K. Montelius, A.O. Karlsson, G. Holmlund, STR data for the AmpFlSTR Identifiler loci from Swedish population in comparison to European, as well as with non-European population, Forensic Sci Int: Genet 2 (2008) e49-e52.

[54] J. Felsentein, Phylogeny inference package (PHYLIP) version 3.6a3, Department of Genetics, University of Washington, Seattle, 2002.

[55] SPSS for Windows, Rel. 11.0.1, SPSS Inc, Chicago, 2001.

[56] J.C. Long, R.C. Williams, J.E. McAuley, R. Meids, R. Partel, M. Tregellas, S.F. South, A.E. Rea, B. McCormick, U. Iwaniec, Genetic variation in Arizona Mexican Americans: estimation and interpretation of admixture proportions, Am J Phys Anthropol 84 (1991) 141-157.

[57] T. Ota, DISPAN: genetic distance and phylogenetic analysis, Institute of Molecular Evolutionary Genetics, Pennsylvania State University, Pennsylvania, 1993.

[58] G. Carmody, G-test, Carleton University, Canada, 1990.

[59] J.K. Pritchard, M. Stephens, P. Donnelly, Inference of population structure using multilocus genotype data, Genetics 155 (2000) 945-959.

[60] E.M. Shepard, R.A. Chow, E. Suafóa, D. Addison, A.M. Perez-Miranda, R.L. Garcia-Bertrand, R.J. Herrera, Autosomal STR variation in five Austronesian populations, Hum Biol 77 (2005) 825-851. 
[61] S.A. Peter, R. Johnson, C. Taylor, A. Hanna, P. Roberts, P. McNeil, B. Archer, C. SinQuee, P. Roberts, The incidence and prevalence of type-1 diabetes mellitus, J Natl Med Assoc 97 (2005) 250-252.

[62] S. Peter, G. McDigean, P. Sandiford, T. Smith, Congenital adrenal hyperplasia in the Bahamas due to 21-hydroxylase deficiency, West Indian Med J 55 (2006) 110-112.

[63] J. Pepin, From the Old World to the New World: an ecologic study of population susceptibility to HIV infection, Trop Med Int Health 10 (2005) 627-639.

[64] K. de Albuquerque, J. McElroy, Bahamian labor migration, 1901-1963. New West Indian Guide 60 (3/4) (1986) 167-203.

[65] D.M. Behar, B. Yunusbayev, M. Metspalu, E. Metspalu, S. Rosset, J. Parik, S. Rootsi, G. Chaubey, I. Kutuev, G. Yudkovsky, E.K. Khusnutdinova, O. Balanovsky, O. Semino, L. Pereira, D. Comas, D. Gurwitz, B. Bonne-Tamir, T. Parfitt, M.F. Hammer, K. Skorecki, R. Villems, The genome-wide structure of the Jewish people, Nature 466 (2010) 238-242.

[66] A. Lawlor, The Eleutherian Adventurers, J Bahamas Hist Soc 20 (1998) 4-9.

[67] H. Johnson, The Bahamas in slavery and freedom, Ian Randle Publishing, Kingston, 1991.

[68] D.M. McCartney, Bahamian culture and factors which impact upon it, Dorrance Publishing Co., Inc., Pennsylvania, 2004.

[69] A. Torroni, C. Rengo, V. Guida, F. Cruciani, D. Sellitto, A. Coppa, F.L. Calderon, B. Simionati, G. Valle, M. Richards, V. Macaulay, R. Scozzari, Do the four clades of the mtDNA haplogroup L2 evolve at different rates?, Am J Hum Genet 69 (2001) 13481356. 


\section{Appendix IV}

Fig. 1: Geographic locations of Abaco (ABA), Eleuthera (ELU), Exuma (EXU), Grand Bahama (GRB) and Long Island (LNG) within the Bahamian archipelago

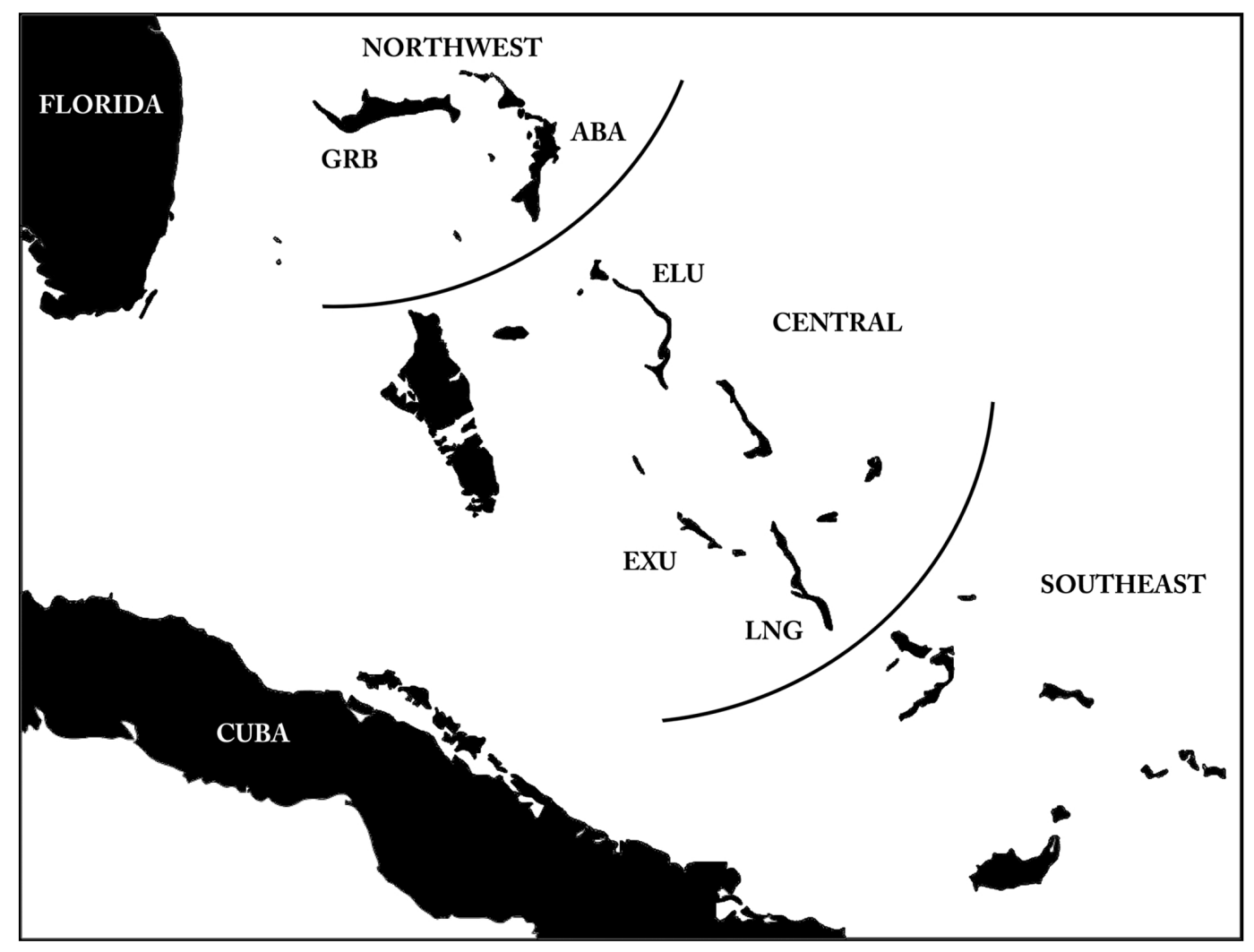


Fig. 2A: Multidimensional Scaling plot $($ Stress $=0.10286)$ of all populations

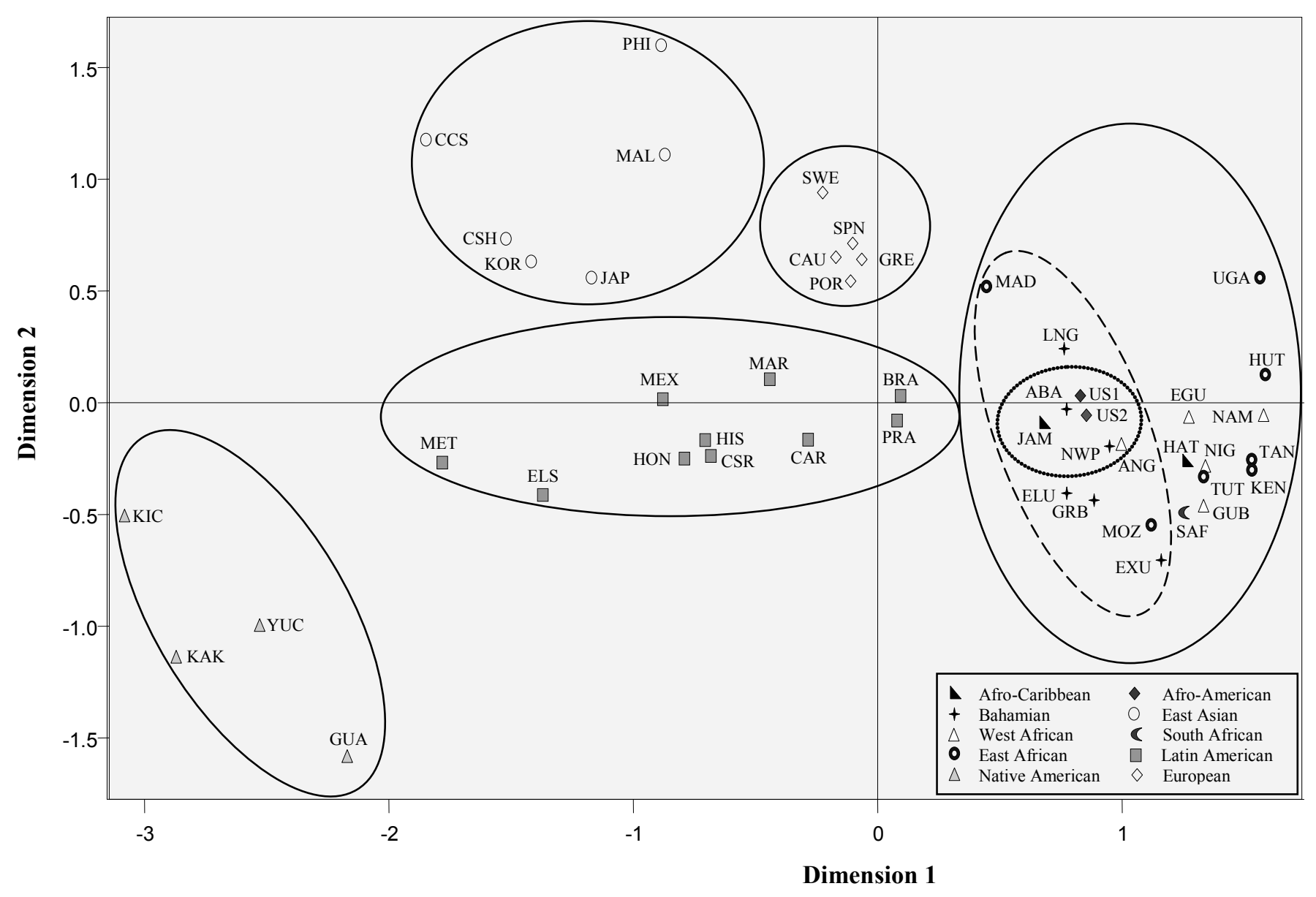


Fig. 2B: Multidimensional Scaling plot (Stress = 0.22533) of African and African-derived collections

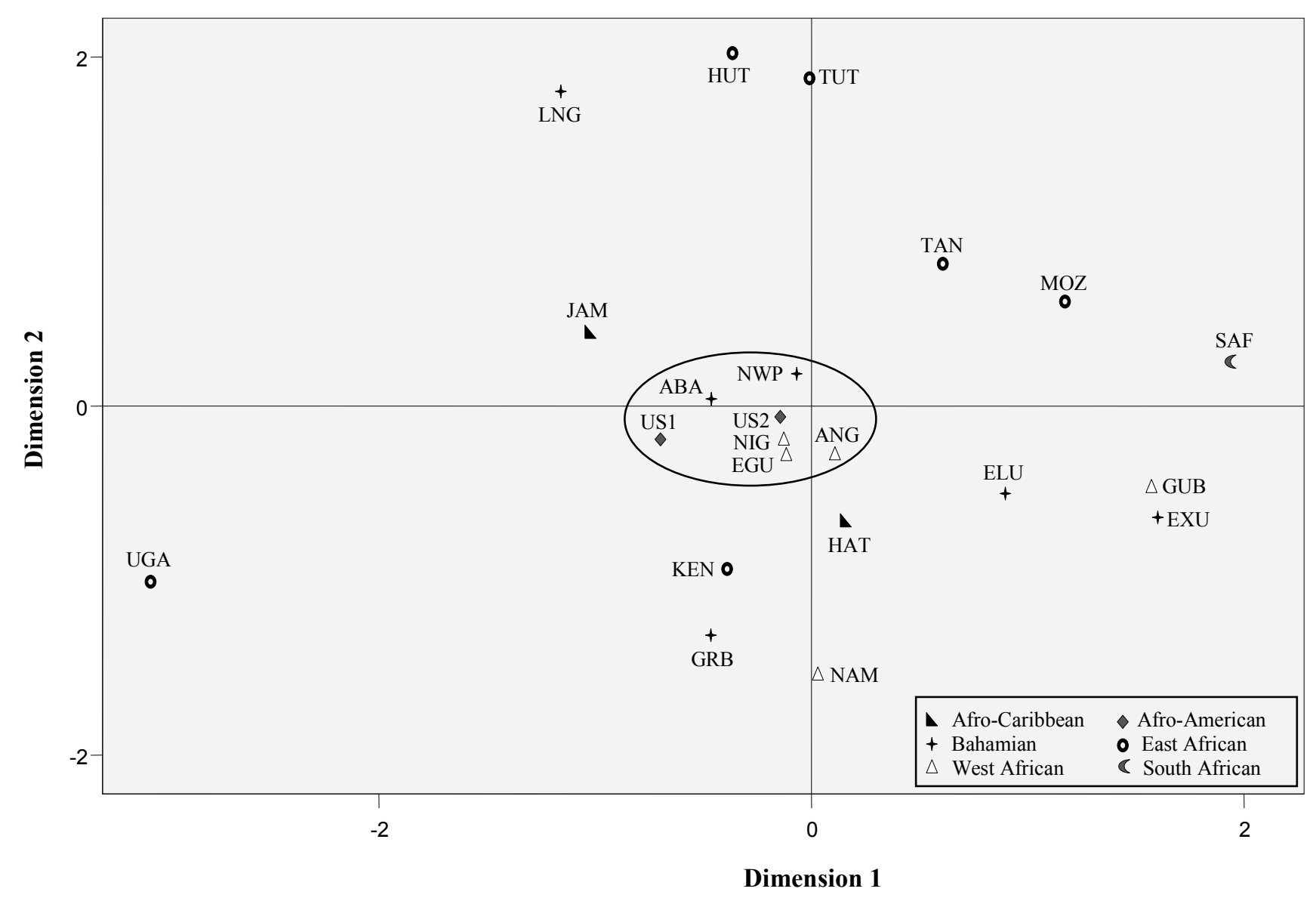




\begin{tabular}{|c|c|c|c|c|}
\hline $\begin{array}{c}\text { Biogeographical } \\
\text { origin }\end{array}$ & Population & Abbreviation & Sample Size & Reference \\
\hline \multirow{6}{*}{ Bahamian } & Abaco & $\mathrm{ABA}$ & 111 & Present study \\
\hline & Eleuthera & ELU & 112 & Present study \\
\hline & Exuma & EXU & 92 & Present study \\
\hline & Grand Bahama & GRB & 133 & Present study \\
\hline & Long Island & LNG & 87 & Present study \\
\hline & New Providence & NWP & 221 & {$[8]$} \\
\hline \multirow[t]{2}{*}{ Afro-Caribbean $\dagger$} & Haiti & HAT & 111 & [9] \\
\hline & Jamaica & JAM & 119 & [9] \\
\hline \multirow[t]{2}{*}{ Afro-American $\dagger$} & US African American 1 (Minnesota) & US1 & 157 & [19] \\
\hline & US African American 2 (General) & US2 & 258 & [20] \\
\hline \multirow{5}{*}{ West African } & Cabinda (Angola) & ANG & 110 & [21] \\
\hline & Guinea-Bissau & GUB & 92 & {$[22]$} \\
\hline & Equatorial Guinea & EGU & 134 & [23] \\
\hline & Namibia & NAM & 195 & [24] \\
\hline & Nigeria & NIG & 337 & {$[25]$} \\
\hline \multirow[t]{7}{*}{ East African } & Hutu (Rwanda) & HUT & 95 & {$[26]$} \\
\hline & Kenya & KEN & 65 & {$[26]$} \\
\hline & Madagascar & MAD & 67 & [27] \\
\hline & Maputo (Mozambique) & $\mathrm{MOZ}$ & 144 & [28] \\
\hline & Tanzania & TAN & 272 & [29] \\
\hline & Tutsi (Rwanda) & TUT & 126 & {$[30]$} \\
\hline & Uganda & UGA & 90 & [31] \\
\hline South African & Cape Town (South Africa) & SAF & 98 & [32] \\
\hline \multirow{6}{*}{ East Asian } & Chao Shan (South China) & $\mathrm{CCS}$ & 144 & [33] \\
\hline & Japan & JAP & 526 & {$[34]$} \\
\hline & Korea & KOR & 231 & {$[35]$} \\
\hline & Malaysia & MAL & 210 & {$[36]$} \\
\hline & Philippines & PHI & 106 & [37] \\
\hline & Shaanxi Han (NW China) & $\mathrm{CSH}$ & 203 & [38] \\
\hline \multirow[t]{4}{*}{ Native American } & Guarani & GUA & 50 & [39] \\
\hline & Kakchikel & KAK & 59 & [40] \\
\hline & K'iche & KIC & 27 & [40] \\
\hline & Yucatan & YUC & 121 & [40] \\
\hline \multirow[t]{10}{*}{ Latin American } & Caracas (Central Venezuela) & CAR & 255 & [41] \\
\hline & Central Mexico & MEX & 211 & [42] \\
\hline & Costa Rica & CSR & 500 & [43] \\
\hline & El Salvador & ELS & 228 & {$[44]$} \\
\hline & Hispanic (US -Minnesota) & HIS & 151 & [19] \\
\hline & Honduras & $\mathrm{HON}$ & 198 & {$[45]$} \\
\hline & Maracaibo (Venezuela) & MAR & 203 & {$[46]$} \\
\hline & Metztitlán (Mexico) & MET & 180 & {$[47]$} \\
\hline & Puerto Rico American (Massachusetts) & PRA & 205 & [48] \\
\hline & Rio de Janeiro (Brazil) & BRA & 300 & [49] \\
\hline \multirow[t]{5}{*}{ European } & Caucasian (US - General) & CAU & 302 & {$[50]$} \\
\hline & Greece & GRE & 300 & {$[51]$} \\
\hline & Portugal & POR & 2125 & {$[52]$} \\
\hline & Spain & SPN & 342 & {$[53]$} \\
\hline & Sweden & SWE & 311 & {$[54]$} \\
\hline
\end{tabular}

$\uparrow$ These groups are combined in some analyses as New World Africans. For specific analyses see Materials \& Methods.

Table 1: Populations analyzed 


\begin{tabular}{|c|c|c|c|c|c|}
\hline Population & No. of Alleles & ${\text { Avg } \mathbf{H}_{\mathbf{e}}}$ CMP & CPD & CPE \\
\hline Abaco & 154 & 0.81441 & $6.0957 \mathrm{E}+17$ & 0.6725596555 & 0.999999803 \\
\hline Eleuthera & 148 & 0.79986 & $1.114 \mathrm{E}+17$ & 0.719034632 & 0.999999408 \\
\hline Exuma & 144 & 0.79131 & $7.2498 \mathrm{E}+16$ & 0.7302691485 & 0.999999554 \\
\hline & 149 & 0.80187 & $4.0009 \mathrm{E}+17$ & 0.6819381436 & 0.999999535 \\
\hline Grand Bahama & 145 & 0.78084 & $3.2122 \mathrm{E}+17$ & 0.6960417459 & 0.999998200 \\
\hline Long Island & & & &
\end{tabular}

Table 2: Parameters of forensic and population genetics interest 


\begin{tabular}{|c|c|c|c|c|c|c|c|c|c|c|c|c|}
\hline \multirow[b]{2}{*}{ Locus } & \multicolumn{4}{|c|}{ Abaco } & \multicolumn{4}{|c|}{ Eleuthera } & \multicolumn{4}{|c|}{ Exuma } \\
\hline & $\mathbf{H}_{\mathbf{o}}$ & $\mathbf{H}_{\mathbf{e}}$ & HWE & $\mathbf{F}_{\text {IS }}$ & $\mathbf{H}_{\mathbf{o}}$ & $\mathbf{H}_{\mathbf{e}}$ & HWE & $\mathbf{F}_{\text {IS }}$ & $\mathbf{H}_{\mathbf{o}}$ & $\mathbf{H}_{\mathbf{e}}$ & HWE & $\mathbf{F}_{\text {IS }}$ \\
\hline D8S1179 & 0.76577 & 0.81301 & 0.80445 & 0.0584 & 0.77679 & 0.75857 & 0.96226 & -0.0241 & 0.72826 & 0.75944 & 0.03445 & 0.0413 \\
\hline D21S11 & 0.90991 & 0.86776 & 0.93121 & -0.0488 & 0.89286 & 0.84910 & 0.09641 & -0.0518 & 0.84783 & 0.84058 & 0.92516 & -0.0087 \\
\hline D7S820 & 0.83784 & 0.79475 & 0.72825 & -0.0545 & 0.76786 & 0.77386 & 0.99972 & 0.0078 & 0.83696 & 0.78908 & 0.43687 & -0.0610 \\
\hline CSF1PO & 0.82883 & 0.77689 & 0.80345 & -0.0672 & 0.74107 & 0.74135 & 0.16399 & 0.0004 & 0.80435 & 0.75493 & 0.71746 & -0.0658 \\
\hline D3S1358 & 0.75676 & 0.75692 & 0.06352 & 0.0002 & 0.80357 & 0.77002 & 0.14962 & -0.0438 & 0.73913 & 0.72054 & 0.41334 & -0.0259 \\
\hline ТH01 & 0.78378 & 0.76870 & 0.51328 & -0.0197 & 0.79464 & 0.78303 & 0.14210 & -0.0149 & 0.73913 & 0.76746 & 0.41596 & 0.0371 \\
\hline D13S317 & 0.78378 & 0.75541 & 0.07326 & -0.0377 & 0.71429 & 0.70992 & 0.14828 & -0.0062 & 0.67391 & 0.69779 & 0.91552 & 0.0344 \\
\hline D16S539 & 0.73874 & 0.80995 & 0.36917 & 0.0883 & 0.76786 & 0.81538 & 0.78695 & 0.0585 & 0.65217 & 0.77251 & 0.14300 & 0.1565 \\
\hline D2S1338 & 0.86486 & 0.89434 & 0.01319 & 0.0331 & 0.87500 & 0.88625 & 0.78298 & 0.0128 & 0.85870 & 0.88673 & 0.60520 & 0.0318 \\
\hline D19S433 & 0.85586 & 0.84379 & 0.95210 & -0.0144 & 0.81250 & 0.83836 & 0.12827 & 0.0310 & 0.85870 & 0.82294 & 0.52092 & -0.0437 \\
\hline vWA & 0.83784 & 0.82165 & 0.40457 & -0.0198 & 0.81250 & 0.79056 & 0.50154 & -0.0279 & 0.70652 & 0.81159 & 0.07931 & 0.1301 \\
\hline TPOX & 0.77477 & 0.77869 & 0.76354 & 0.0050 & 0.75676 & 0.77726 & 0.72729 & 0.0265 & 0.84783 & 0.79205 & 0.65367 & -0.0708 \\
\hline D18S51 & 0.88288 & 0.88158 & 0.86188 & -0.0015 & 0.89286 & 0.88837 & 0.24703 & -0.0051 & 0.85870 & 0.86636 & 0.66511 & 0.0089 \\
\hline D5S818 & 0.75676 & 0.76503 & 0.74637 & 0.0109 & 0.78571 & 0.74752 & 0.47691 & -0.0513 & 0.77174 & 0.77798 & 0.33374 & 0.0081 \\
\hline FGA & 0.83784 & 0.86727 & 0.10227 & 0.0341 & 0.80357 & 0.87748 & 0.16620 & 0.0846 & 0.94565 & 0.88952 & 0.99769 & -0.0635 \\
\hline
\end{tabular}

HWE $P$ values in italics are $<\boldsymbol{\alpha}=0.05 ; \mathrm{F}_{\text {IS }}$ values in bold represent associated $P$ values $<\boldsymbol{\alpha}=0.05$

Table 3: Intra-population diversity indices for the Bahamian populations 


\begin{tabular}{|c|c|c|c|c|c|c|c|c|}
\hline \multirow[b]{2}{*}{ Locus } & \multicolumn{4}{|c|}{ Grand Bahama } & \multicolumn{4}{|c|}{ Long Island } \\
\hline & $\mathbf{H}_{\mathrm{o}}$ & $\mathbf{H}_{\mathbf{e}}$ & HWE & $\mathbf{F}_{\text {IS }}$ & $\mathbf{H}_{\mathrm{o}}$ & $\mathbf{H}_{\mathrm{e}}$ & HWE & $F_{\text {IS }}$ \\
\hline D8S1179 & 0.80451 & 0.79671 & 0.87995 & -0.0098 & 0.71264 & 0.82001 & 0.02718 & 0.1316 \\
\hline D21S11 & 0.84962 & 0.84091 & 0.32556 & -0.0104 & 0.78161 & 0.82971 & 0.62111 & 0.0583 \\
\hline D7S820 & 0.80451 & 0.79765 & 0.36357 & -0.0086 & 0.85057 & 0.79158 & 0.95176 & -0.0750 \\
\hline CSF1PO & 0.77444 & 0.78539 & 0.66134 & 0.0140 & 0.74713 & 0.75497 & 0.32754 & 0.0104 \\
\hline D3S1358 & 0.71429 & 0.73993 & 0.56191 & 0.0348 & 0.60920 & 0.72912 & 0.05238 & 0.1653 \\
\hline TH01 & 0.80451 & 0.76164 & 0.20138 & -0.0565 & 0.74713 & 0.77889 & 0.12645 & 0.0410 \\
\hline D13S317 & 0.66165 & 0.72799 & 0.11995 & 0.0914 & 0.77011 & 0.77782 & 0.48606 & 0.0100 \\
\hline D16S539 & 0.80451 & 0.79665 & 0.75466 & -0.0099 & 0.83908 & 0.79682 & 0.67838 & -0.0534 \\
\hline D2S1338 & 0.89474 & 0.89604 & 0.80579 & 0.0015 & 0.82759 & 0.88958 & 0.76268 & 0.0701 \\
\hline D19S433 & 0.86466 & 0.87093 & 0.30027 & 0.0072 & 0.83908 & 0.86639 & 0.02676 & 0.0317 \\
\hline vWA & 0.84962 & 0.82241 & 0.30851 & -0.0332 & 0.79310 & 0.82440 & 0.36944 & 0.0382 \\
\hline TPOX & 0.78195 & 0.77568 & 0.34062 & -0.0081 & 0.75862 & 0.76766 & 0.26181 & 0.0118 \\
\hline D18S51 & 0.81203 & 0.87090 & 0.16695 & 0.0678 & 0.85057 & 0.89529 & 0.26791 & 0.0502 \\
\hline D5S818 & 0.74242 & 0.75029 & 0.98880 & 0.0105 & 0.71264 & 0.72693 & 0.54526 & 0.0198 \\
\hline FGA & 0.86466 & 0.85816 & 0.26612 & -0.0076 & 0.87356 & 0.89649 & 0.04548 & 0.0257 \\
\hline
\end{tabular}

HWE $P$ values in italics are $<\alpha=0.05 ; F_{\text {IS }}$ values in bold represent associated $P$ values $<\boldsymbol{\alpha}=0.05$

Table 3: Intra-population diversity indices for the Bahamian populations (Cont'd) 


\begin{tabular}{c}
\hline \\
\hline Locus \\
\hline D8S1179 \\
D21S11 \\
D7S820 \\
CSF1PO \\
D3S1358 \\
TH01 \\
D13S317 \\
D16S539 \\
D2S1338 \\
D19S433 \\
vWA \\
TPOX \\
D18S51 \\
D5S818 \\
FGA \\
All Loci
\end{tabular}

\begin{tabular}{ccc}
\hline \multicolumn{3}{c}{ Bahamian } \\
\hline $\mathbf{G}_{\text {st }}$ & $\mathbf{H}_{\mathbf{s}}$ & $\mathbf{H}_{\mathbf{t}}$ \\
\hline 0.00616 & 0.78811 & 0.79299 \\
0.00654 & 0.84166 & 0.84720 \\
0.00622 & 0.78457 & 0.78948 \\
0.00613 & 0.76285 & 0.76756 \\
0.00904 & 0.74247 & 0.74924 \\
0.00286 & 0.76538 & 0.76758 \\
0.00534 & 0.72914 & 0.73306 \\
0.00856 & 0.79550 & 0.80237 \\
0.00596 & 0.88636 & 0.89167 \\
0.00423 & 0.84150 & 0.84507 \\
0.00496 & 0.81016 & 0.81420 \\
0.00471 & 0.77575 & 0.77941 \\
0.00640 & 0.87624 & 0.88188 \\
0.00438 & 0.74986 & 0.75316 \\
0.00590 & 0.87483 & 0.88003 \\
0.00583 & 0.80162 & 0.80633 \\
\hline
\end{tabular}

\begin{tabular}{ccc}
\hline \multicolumn{3}{c}{ New World African $\dagger$} \\
\hline $\mathbf{G}_{\text {st }}$ & $\mathbf{H}_{\mathbf{s}}$ & $\mathbf{H}_{\mathbf{t}}$ \\
\hline 0.00552 & 0.78764 & 0.79201 \\
0.00243 & 0.84011 & 0.84216 \\
0.00501 & 0.77856 & 0.78248 \\
0.00192 & 0.78062 & 0.78212 \\
0.00378 & 0.75108 & 0.75393 \\
0.00521 & 0.73590 & 0.73975 \\
0.00229 & 0.70197 & 0.70358 \\
0.00295 & 0.79262 & 0.79497 \\
0.00242 & 0.88744 & 0.88959 \\
0.00209 & 0.84433 & 0.84609 \\
0.00335 & 0.81387 & 0.81661 \\
0.00538 & 0.75695 & 0.76105 \\
0.00197 & 0.87834 & 0.88007 \\
0.00220 & 0.75069 & 0.75234 \\
0.00400 & 0.86767 & 0.87115 \\
0.00334 & 0.79785 & 0.80053
\end{tabular}

\begin{tabular}{ccc}
\hline \multicolumn{3}{c}{ African } \\
\hline $\mathbf{G}_{\text {st }}$ & $\mathbf{H}_{\mathbf{s}}$ & $\mathbf{H}_{\mathbf{t}}$ \\
\hline 0.01168 & 0.77586 & 0.78503 \\
0.01012 & 0.85178 & 0.86048 \\
0.00853 & 0.76342 & 0.76999 \\
0.01376 & 0.77664 & 0.78748 \\
0.01152 & 0.73082 & 0.73934 \\
0.01320 & 0.72643 & 0.73614 \\
0.01077 & 0.71109 & 0.71883 \\
0.00925 & 0.78909 & 0.79645 \\
0.01026 & 0.88062 & 0.88975 \\
0.01130 & 0.83199 & 0.84150 \\
0.00967 & 0.81619 & 0.82416 \\
0.02079 & 0.76301 & 0.77921 \\
0.00690 & 0.86610 & 0.87211 \\
0.00974 & 0.75268 & 0.76008 \\
0.00859 & 0.87488 & 0.88246 \\
0.01100 & 0.79404 & 0.80287 \\
\hline
\end{tabular}

\begin{tabular}{ccc}
\hline \multicolumn{3}{c}{ Asian } \\
\hline $\mathbf{G}_{\text {st }}$ & $\mathbf{H}_{\mathbf{s}}$ & $\mathbf{H}_{\mathbf{t}}$ \\
\hline 0.00762 & 0.84279 & 0.84926 \\
0.00871 & 0.81690 & 0.82408 \\
0.00944 & 0.75886 & 0.76608 \\
0.00874 & 0.72283 & 0.72920 \\
0.01191 & 0.71936 & 0.72803 \\
0.01452 & 0.69914 & 0.70944 \\
0.00608 & 0.80024 & 0.80513 \\
0.00957 & 0.77487 & 0.78235 \\
0.00706 & 0.86761 & 0.87377 \\
0.01380 & 0.79961 & 0.81079 \\
0.00681 & 0.79667 & 0.80213 \\
0.00567 & 0.62619 & 0.62976 \\
0.00742 & 0.85343 & 0.85981 \\
0.00761 & 0.78432 & 0.79033 \\
0.00468 & 0.85688 & 0.86091 \\
0.00858 & 0.78131 & 0.78807 \\
\hline
\end{tabular}

$\uparrow$ The New World African assemblage includes the Afro-Caribbean and Afro-American groups of populations

Table 4: Inter-, intra- and total population genetic variance 


\begin{tabular}{c}
\hline \\
\hline Locus \\
\hline D8S1179 \\
D21S11 \\
D7S820 \\
CSF1PO \\
D3S1358 \\
TH01 \\
D13S317 \\
D16S539 \\
D2S1338 \\
D19S433 \\
vWA \\
TPOX \\
D18S51 \\
D5S818 \\
FGA \\
All Loci \\
\hline
\end{tabular}

\begin{tabular}{ccc}
\hline \multicolumn{3}{c}{ European } \\
\hline $\mathbf{G}_{\mathbf{s t}}$ & $\mathbf{H}_{\mathbf{s}}$ & $\mathbf{H}_{\mathbf{t}}$ \\
\hline 0.00258 & 0.81289 & 0.81499 \\
0.00410 & 0.83263 & 0.83606 \\
0.00253 & 0.80819 & 0.81024 \\
0.00213 & 0.71876 & 0.72029 \\
0.00198 & 0.78931 & 0.79088 \\
0.00842 & 0.77844 & 0.78505 \\
0.00301 & 0.78118 & 0.78353 \\
0.00569 & 0.77226 & 0.77668 \\
0.00324 & 0.86862 & 0.87145 \\
0.00340 & 0.78286 & 0.78553 \\
0.00187 & 0.80861 & 0.81012 \\
0.00465 & 0.61984 & 0.62273 \\
0.00244 & 0.87438 & 0.87652 \\
0.00293 & 0.70337 & 0.70544 \\
0.00206 & 0.85976 & 0.86154 \\
0.00337 & 0.78741 & 0.79007 \\
\hline
\end{tabular}

\begin{tabular}{ccc}
\hline \multicolumn{3}{c}{ Latin American } \\
\hline $\mathbf{G}_{\text {st }}$ & $\mathbf{H}_{\mathbf{s}}$ & $\mathbf{H}_{\mathbf{t}}$ \\
\hline 0.00480 & 0.79695 & 0.80079 \\
0.00377 & 0.83353 & 0.83669 \\
0.00771 & 0.78777 & 0.79389 \\
0.00312 & 0.72011 & 0.72236 \\
0.01391 & 0.74930 & 0.75987 \\
0.01688 & 0.76321 & 0.77631 \\
0.01044 & 0.81336 & 0.82195 \\
0.00741 & 0.78106 & 0.78689 \\
0.00897 & 0.86660 & 0.87444 \\
0.00799 & 0.82660 & 0.83326 \\
0.00790 & 0.78317 & 0.78941 \\
0.00875 & 0.67422 & 0.68017 \\
0.00344 & 0.87671 & 0.87974 \\
0.01129 & 0.71450 & 0.72266 \\
0.00592 & 0.87427 & 0.87948 \\
0.00807 & 0.79076 & 0.79719 \\
\hline
\end{tabular}

\begin{tabular}{ccc}
\hline \multicolumn{3}{c}{ Native American } \\
\hline $\mathbf{G}_{\mathbf{s t}}$ & $\mathbf{H}_{\mathbf{s}}$ & $\mathbf{H}_{\mathbf{t}}$ \\
\hline 0.04425 & 0.72114 & 0.75453 \\
0.02130 & 0.78235 & 0.79937 \\
0.02102 & 0.69099 & 0.70583 \\
0.00543 & 0.69694 & 0.70075 \\
0.00406 & 0.64577 & 0.64841 \\
0.00940 & 0.59100 & 0.59660 \\
0.02490 & 0.79415 & 0.81443 \\
0.06529 & 0.72020 & 0.77051 \\
0.02276 & 0.80745 & 0.82625 \\
0.01867 & 0.81190 & 0.82735 \\
0.01685 & 0.71646 & 0.72874 \\
0.06050 & 0.61551 & 0.65514 \\
0.01180 & 0.83139 & 0.84132 \\
0.02495 & 0.62816 & 0.64423 \\
0.01096 & 0.85179 & 0.86123 \\
0.02412 & 0.72701 & 0.74498 \\
\hline
\end{tabular}

\begin{tabular}{ccc}
\hline \multicolumn{3}{c}{ All populations } \\
\hline $\mathbf{G}_{\text {st }}$ & $\mathbf{H}_{\mathbf{s}}$ & $\mathbf{H}_{\mathbf{t}}$ \\
\hline 0.02339 & 0.79043 & 0.80936 \\
0.02398 & 0.83360 & 0.85408 \\
0.03066 & 0.77045 & 0.79482 \\
0.01495 & 0.74407 & 0.75536 \\
0.02005 & 0.73539 & 0.75044 \\
0.06824 & 0.73047 & 0.78397 \\
0.04547 & 0.75926 & 0.79543 \\
0.02227 & 0.77924 & 0.79699 \\
0.02256 & 0.87001 & 0.89009 \\
0.02240 & 0.82224 & 0.84109 \\
0.02207 & 0.79682 & 0.81481 \\
0.04403 & 0.70129 & 0.73359 \\
0.01961 & 0.86698 & 0.88432 \\
0.04563 & 0.73265 & 0.76768 \\
0.01470 & 0.86840 & 0.88135 \\
0.02897 & 0.78675 & 0.81023 \\
\hline
\end{tabular}

Table 4: Inter-, intra- and total population genetic variance (Cont'd) 


\begin{tabular}{|c|c|c|c|c|c|c|}
\cline { 2 - 7 } \multicolumn{1}{c|}{} & \multicolumn{5}{c|}{ Hybrid population } \\
\hline Parental Groups & Long Island & Abaco & New Providence & Eleuthera & Grand Bahama & Exuma \\
\hline African & $0.654 \pm 0.029$ & $0.750 \pm 0.019$ & $0.797 \pm 0.016$ & $0.803 \pm 0.022$ & $0.867 \pm 0.023$ & $0.904 \pm 0.025$ \\
\hline East Asian & $0.077 \pm 0.033$ & $0.015 \pm 0.022$ & $0.000 \pm 0.018$ & $0.000 \pm 0.025$ & $0.005 \pm 0.026$ & $0.000 \pm 0.029$ \\
\hline European & $0.269 \pm 0.036$ & $0.235 \pm 0.024$ & $0.196 \pm 0.019$ & $0.137 \pm 0.027$ & $0.089 \pm 0.028$ & $0.037 \pm 0.031$ \\
\hline Native American & $0.000 \pm 0.023$ & $0.000 \pm 0.015$ & $0.006 \pm 0.012$ & $0.060 \pm 0.017$ & $0.039 \pm 0.018$ & $0.059 \pm 0.020$ \\
\hline
\end{tabular}

Table 5: Admixture proportions generated using grouped parental sources

\begin{tabular}{|c|c|c|c|c|c|c|c|}
\hline$K=3$ & & \multicolumn{4}{|c|}{ Inferred Cluster } & & Exuma \\
\hline & African & 0.594 & 0.625 & 0.747 & 0.724 & 0.771 & 0.801 \\
\hline & European & 0.285 & 0.245 & 0.172 & 0.176 & 0.156 & 0.115 \\
\hline & East Asian/Native American & 0.121 & 0.103 & 0.081 & 0.100 & 0.073 & 0.084 \\
\hline$K=4$ & Inferred Cluster & Long Island & Abaco & New Providence & Eleuthera & Grand Bahama & Exuma \\
\hline & African & 0.532 & 0.611 & 0.702 & 0.677 & 0.722 & 0.753 \\
\hline & European & 0.206 & 0.168 & 0.138 & 0.140 & 0.114 & 0.093 \\
\hline & East Asian & 0.214 & 0.170 & 0.109 & 0.125 & 0.120 & 0.101 \\
\hline & Native American & 0.048 & 0.051 & 0.138 & 0.058 & 0.044 & 0.053 \\
\hline
\end{tabular}

Table 6: Membership proportions generated by Structure 
Supplementary Figure 1: Histograms comparing allelic distributions in the five Bahamian populations surveyed
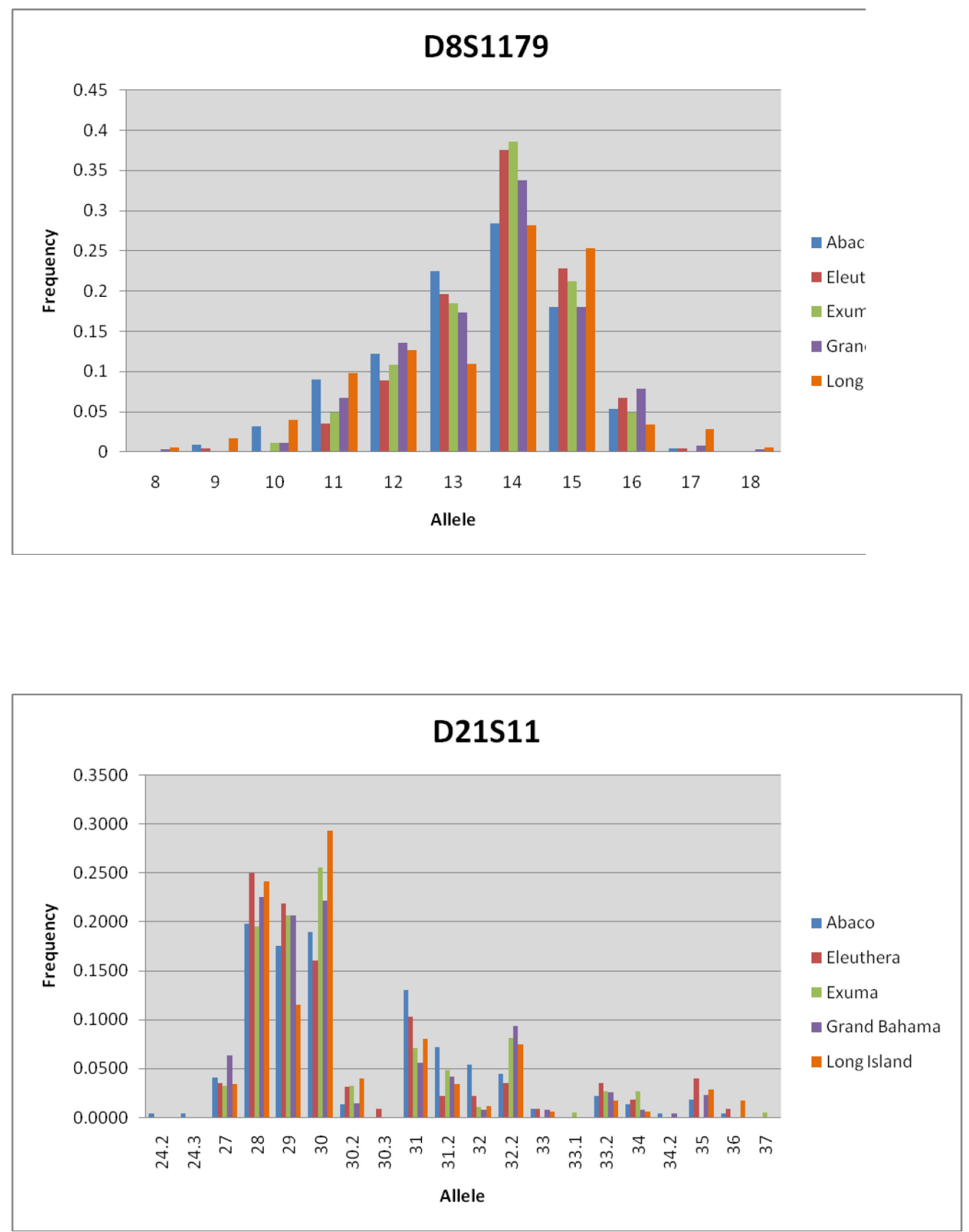

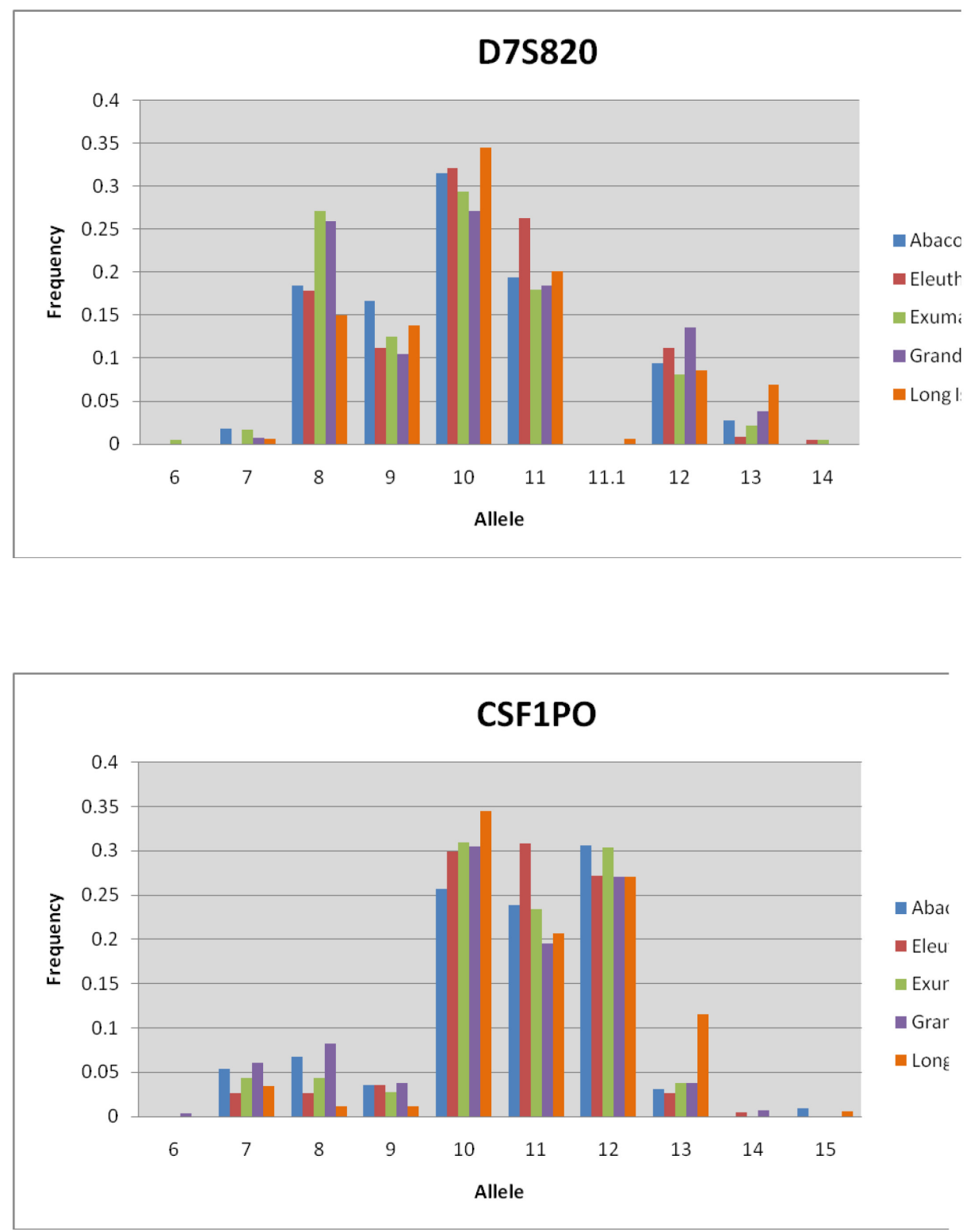

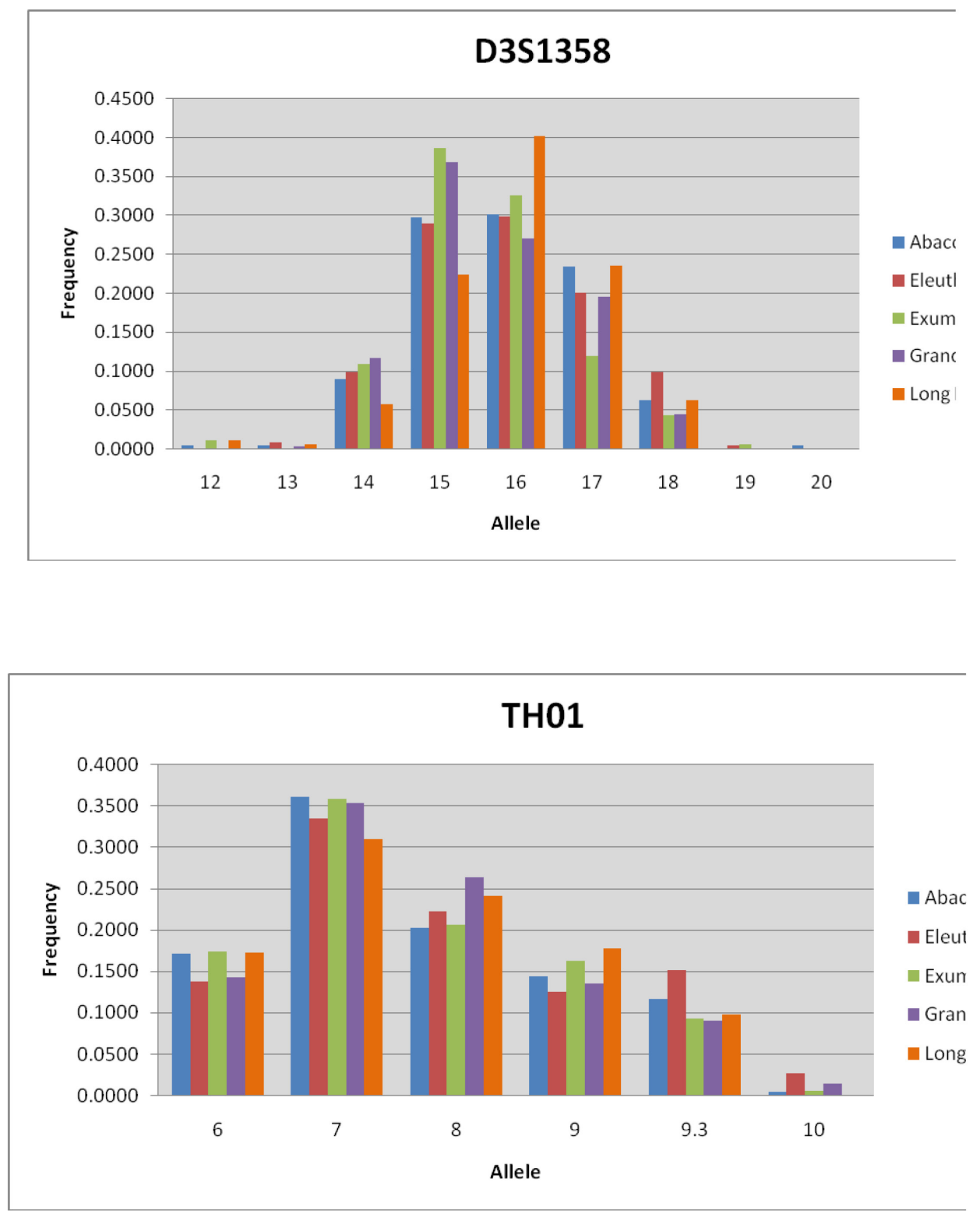

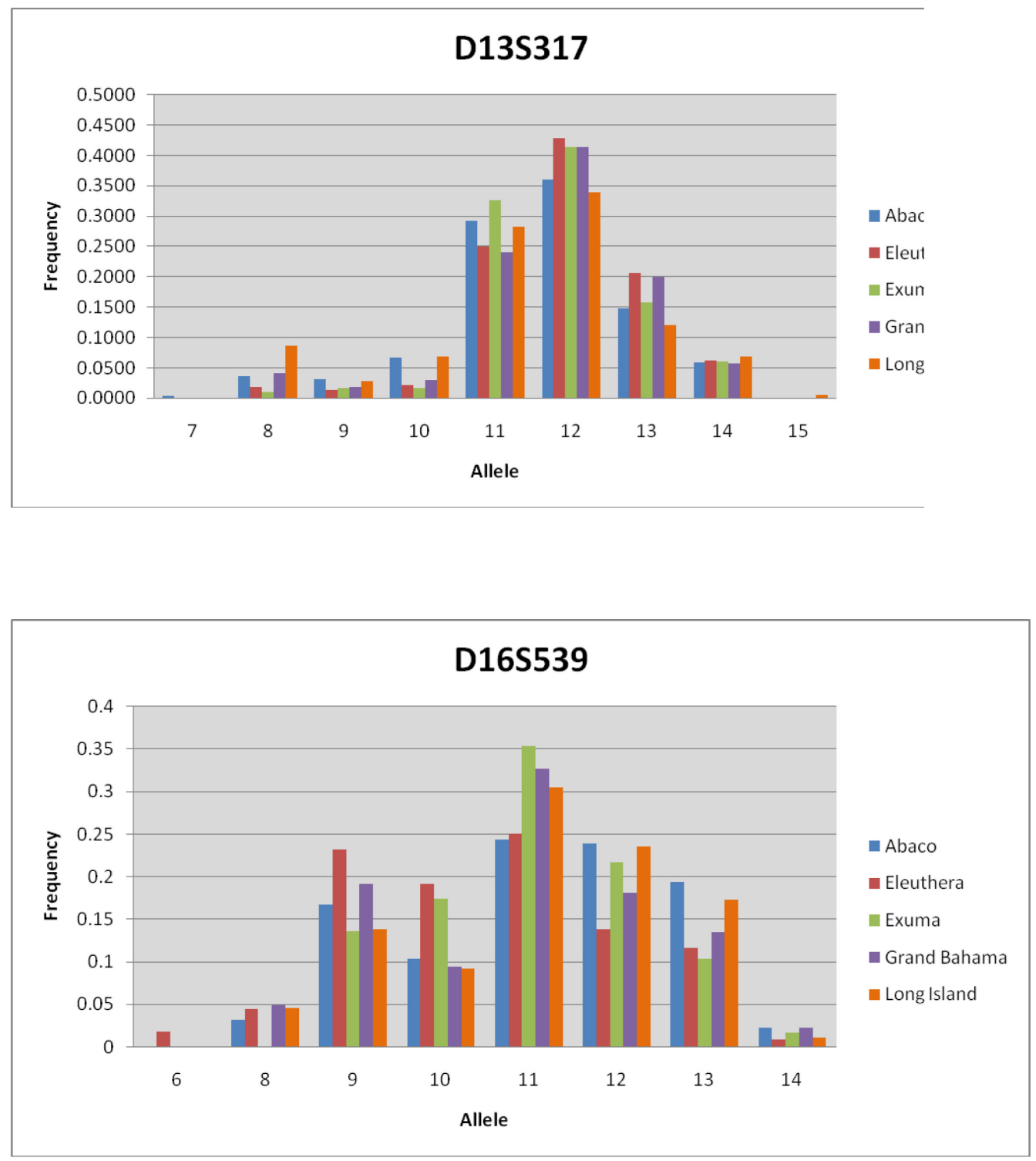

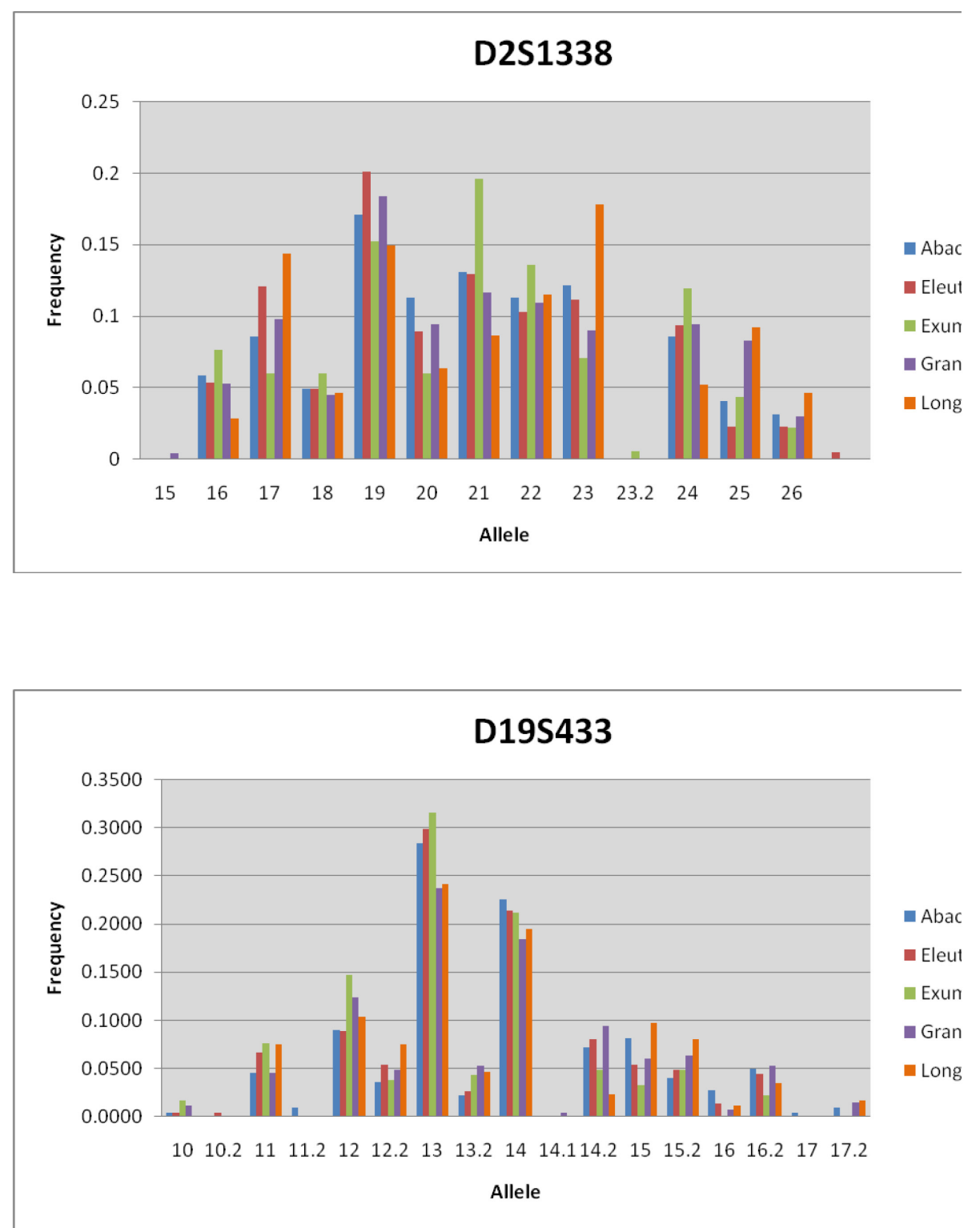

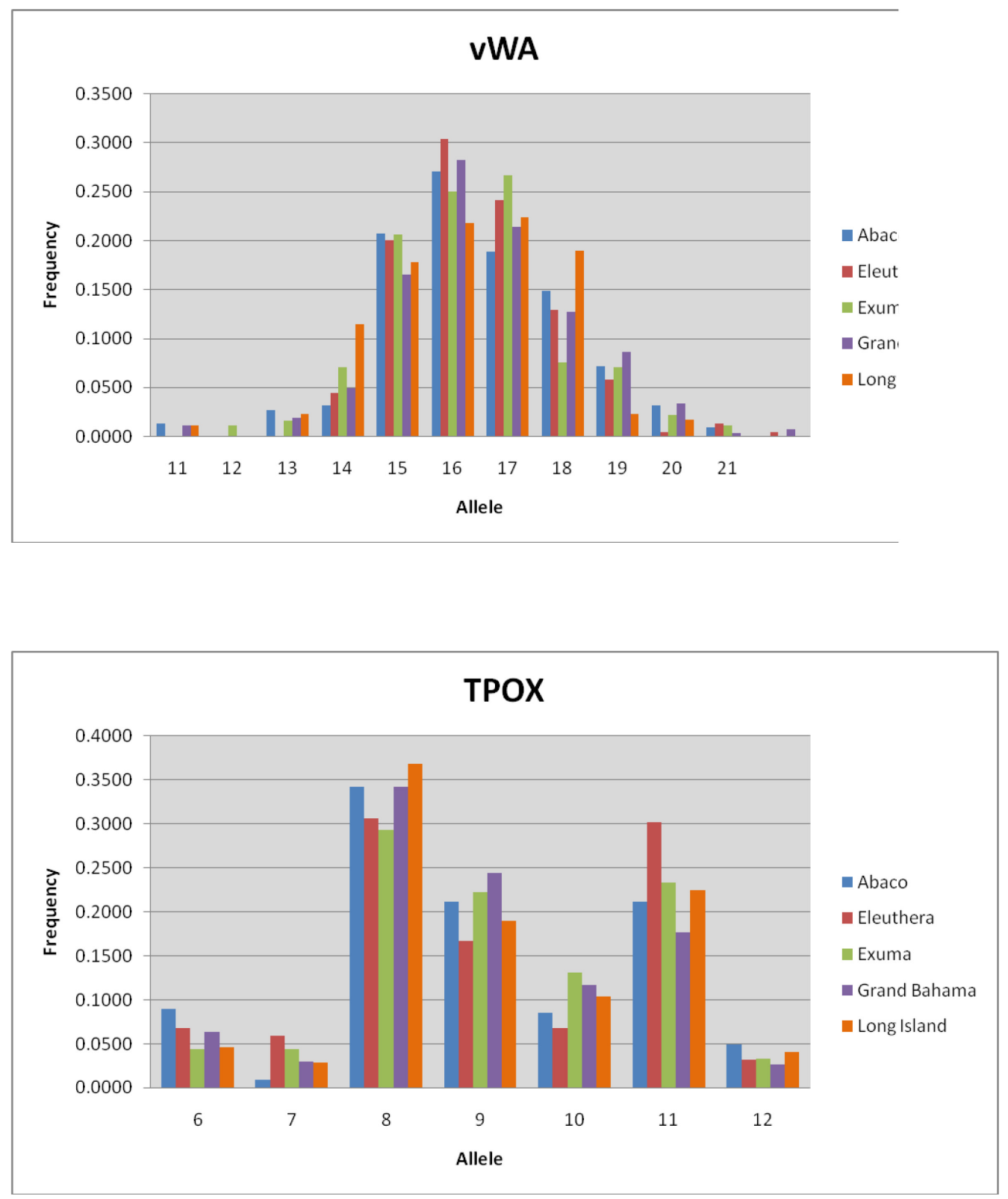

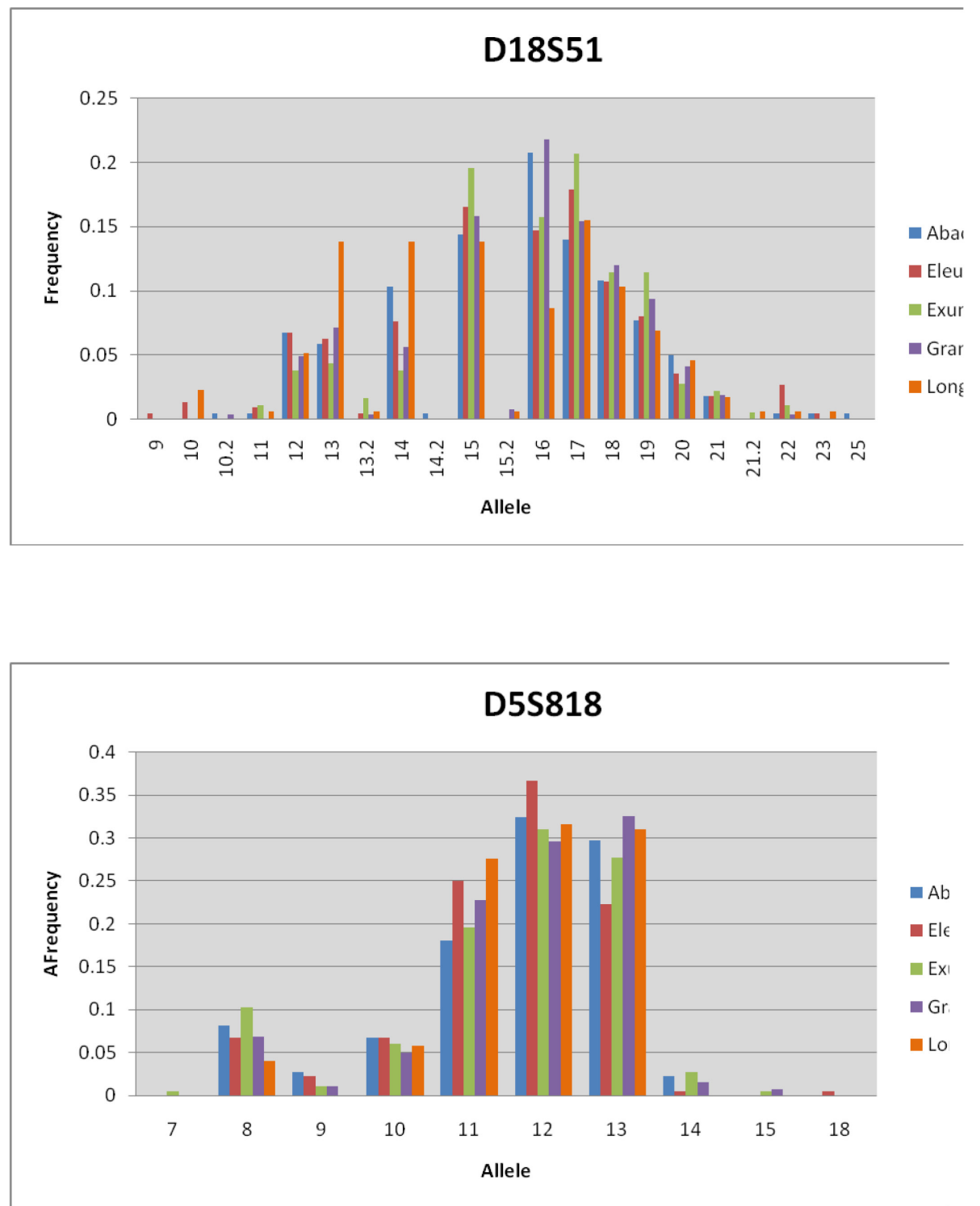


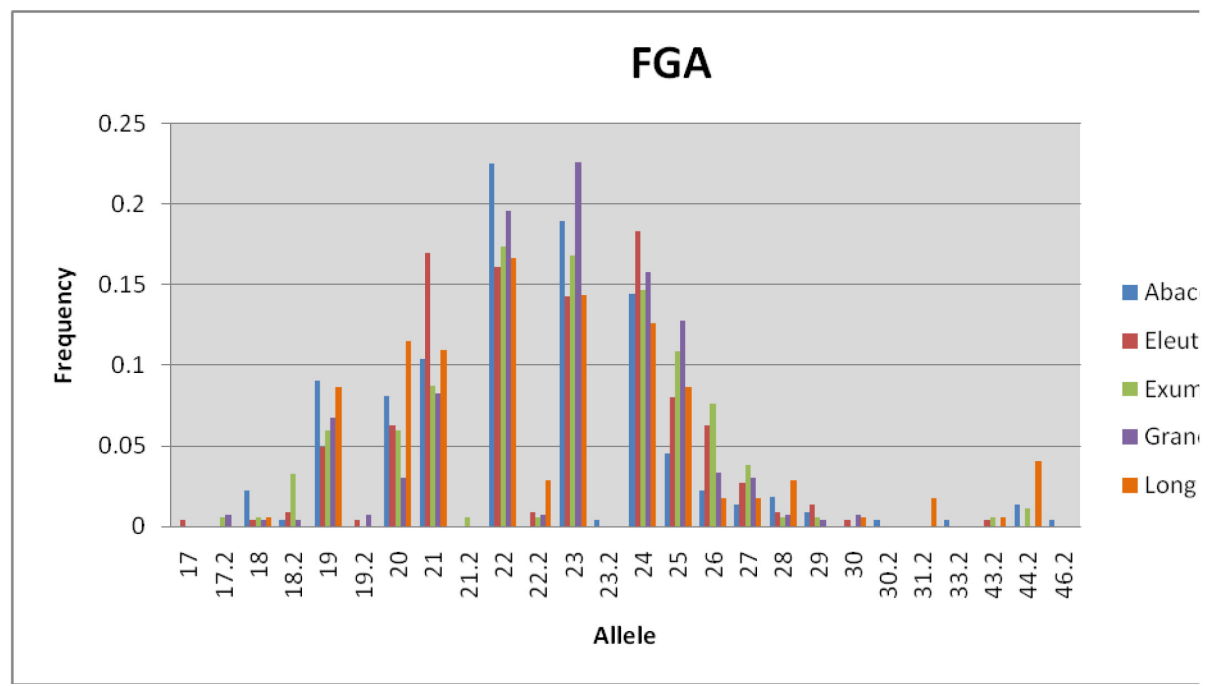


Supplementary Figure 2: Structure bar plots assuming three $(K=3)$ and four $(K=4)$ ancestral populations

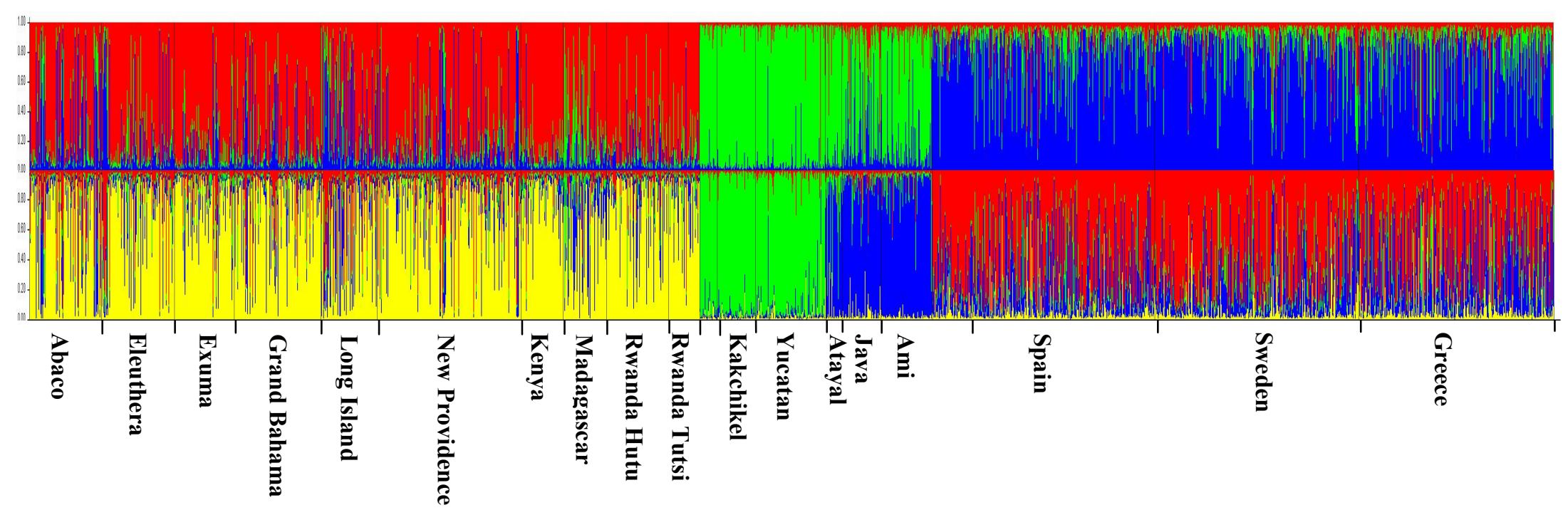




\begin{tabular}{|c|c|c|c|c|c|c|c|c|c|c|c|c|c|c|}
\hline & & \multicolumn{13}{|c|}{ Allele } \\
\hline Locus & $\mathrm{n}$ & 6 & 7 & 8 & 9 & 9.3 & 10 & 10.2 & 11 & 11.2 & 12 & 12.2 & 13 & 13.2 \\
\hline D8S1179 & 111 & & & & 0.0090 & & 0.0315 & & 0.0901 & & 0.1216 & & 0.2252 & \\
\hline D21S11 & 111 & & & & & & & & & & & & & \\
\hline D7S820 & 111 & & 0.0180 & 0.1847 & 0.1667 & & 0.3153 & & 0.1937 & & 0.0946 & & 0.0270 & \\
\hline CSF1PO & 111 & & 0.0541 & 0.0676 & 0.0360 & & 0.2568 & & 0.2387 & & 0.3063 & & 0.0315 & \\
\hline D3S1358 & 111 & & & & & & & & & & 0.0045 & & 0.0045 & \\
\hline TH01 & 111 & 0.1712 & 0.3604 & 0.2027 & 0.1441 & 0.1171 & 0.0045 & & & & & & & \\
\hline D13S317 & 111 & & 0.0045 & 0.0360 & 0.0315 & & 0.0676 & & 0.2928 & & 0.3604 & & 0.1486 & \\
\hline D16S539 & 111 & & & 0.0315 & 0.1667 & & 0.1036 & & 0.2432 & & 0.2387 & & 0.1937 & \\
\hline D2S1338 & 111 & & & & & & & & & & & & & \\
\hline D19S433 & 111 & & & & & & 0.0045 & & 0.0450 & 0.0090 & 0.0901 & 0.0360 & 0.2838 & 0.0225 \\
\hline vWA & 111 & & & & & & & & 0.0135 & & & & 0.0270 & \\
\hline TPOX & 111 & 0.0901 & 0.0090 & 0.3423 & 0.2117 & & 0.0856 & & 0.2117 & & 0.0495 & & & \\
\hline D18S51 & 111 & & & & & & & 0.0045 & 0.0045 & & 0.0676 & & 0.0586 & \\
\hline D5S818 & 111 & & & 0.0811 & 0.0270 & & 0.0676 & & 0.1802 & & 0.3243 & & 0.2973 & \\
\hline FGA & 111 & & & & & & & & & & & & & \\
\hline
\end{tabular}

\begin{tabular}{|c|c|c|c|c|c|c|c|c|c|c|c|c|c|c|}
\hline \multirow[b]{2}{*}{ Locus } & \multirow[b]{2}{*}{$\mathrm{n}$} & \multicolumn{13}{|c|}{ Allele } \\
\hline & & 14 & 14.2 & 15 & 15.2 & 16 & 16.2 & 17 & 17.2 & 18 & 18.2 & 19 & 20 & 21 \\
\hline D8S1179 & 111 & 0.2838 & & 0.1802 & & 0.0541 & & 0.0045 & & & & & & \\
\hline D21S11 & 111 & & & & & & & & & & & & & \\
\hline D7S820 & 111 & & & & & & & & & & & & & \\
\hline CSF1PO & 111 & & & 0.0090 & & & & & & & & & & \\
\hline D3S1358 & 111 & 0.0901 & & 0.2973 & & 0.3018 & & 0.2342 & & 0.0631 & & & 0.0045 & \\
\hline TH01 & 111 & & & & & & & & & & & & & \\
\hline D13S317 & 111 & 0.0586 & & & & & & & & & & & & \\
\hline D16S539 & 111 & 0.0225 & & & & & & & & & & & & \\
\hline D2S1338 & 111 & & & & & 0.0586 & & 0.0856 & & 0.0495 & & 0.1712 & 0.1126 & 0.1306 \\
\hline D19S433 & 111 & 0.2252 & 0.0721 & 0.0811 & 0.0405 & 0.0270 & 0.0495 & 0.0045 & 0.0090 & & & & & \\
\hline vWA & 111 & 0.0315 & & 0.2072 & & \begin{tabular}{|l}
0.2703 \\
\end{tabular} & & 0.1892 & & 0.1486 & & 0.0721 & \begin{tabular}{|l|}
0.0315 \\
\end{tabular} & 0.0090 \\
\hline TPOX & 111 & & & & & & & & & & & & & \\
\hline D18S51 & 111 & 0.1036 & 0.0045 & 0.1441 & & 0.2072 & & 0.1396 & & 0.1081 & & 0.0766 & 0.0495 & 0.0180 \\
\hline D5S818 & 111 & 0.0225 & & & & & & & & & & & & \\
\hline FGA & 111 & & & & & & & & & 0.0225 & 0.0045 & 0.0901 & 0.0811 & 0.1036 \\
\hline
\end{tabular}

\begin{tabular}{|c|c|c|c|c|c|c|c|c|c|c|c|c|c|c|}
\hline & & & & & & & & Allele & & & & & & \\
\hline Locus & $\mathrm{n}$ & 22 & 23 & 23.2 & 24 & 24.2 & 24.3 & 25 & 26 & 27 & 28 & 29 & 30 & 30.2 \\
\hline D8S1179 & 111 & & & & & & & & & & & & & \\
\hline D21S11 & 111 & & & & & 0.0045 & 0.0045 & & & 0.0405 & 0.1982 & 0.1757 & 0.1892 & 0.0135 \\
\hline D7S820 & 111 & & & & & & & & & & & & & \\
\hline CSF1PO & 111 & & & & & & & & & & & & & \\
\hline D3S1358 & 111 & & & & & & & & & & & & & \\
\hline TH01 & 111 & & & & & & & & & & & & & \\
\hline D13S317 & 111 & & & & & & & & & & & & & \\
\hline D16S539 & 111 & & & & & & & & & & & & & \\
\hline D2S1338 & 111 & 0.1126 & 0.1216 & & 0.0856 & & & 0.0405 & 0.0315 & & & & & \\
\hline D19S433 & 111 & & & & & & & & & & & & & \\
\hline vWA & 111 & & & & & & & & & & & & & \\
\hline TPOX & 111 & & & & & & & & & & & & & \\
\hline D18S51 & 111 & 0.0045 & 0.0045 & & & & & 0.0045 & & & & & & \\
\hline D5S818 & 111 & & & & & & & & & & & & & \\
\hline FGA & 111 & 0.2252 & 0.1892 & 0.0045 & 0.1441 & & & 0.0450 & 0.0225 & 0.0135 & 0.0180 & 0.0090 & & 0.0045 \\
\hline
\end{tabular}

Supplementary Table 1: Allelic Frequencies for Abaco 


\begin{tabular}{|c|c|c|c|c|c|c|c|c|c|c|c|c|c|}
\hline \multirow[b]{2}{*}{ Locus } & \multirow[b]{2}{*}{$n$} & \multicolumn{12}{|c|}{ Allele } \\
\hline & & 31 & 31.2 & 32 & 32.2 & 33 & 33.2 & 34 & 34.2 & 35 & 36 & 44.2 & 46.2 \\
\hline D8S1179 & 111 & & & & & & & & & & & & \\
\hline D21S11 & 111 & 0.1306 & 0.0721 & 0.0541 & 0.0450 & 0.0090 & 0.0225 & 0.0135 & 0.0045 & 0.0180 & 0.0045 & & \\
\hline D7S820 & 111 & & & & & & & & & & & & \\
\hline CSF1PO & 111 & & & & & & & & & & & & \\
\hline D3S1358 & 111 & & & & & & & & & & & & \\
\hline TH01 & 111 & & & & & & & & & & & & \\
\hline D13S317 & 111 & & & & & & & & & & & & \\
\hline D16S539 & 111 & & & & & & & & & & & & \\
\hline D2S1338 & 111 & & & & & & & & & & & & \\
\hline D19S433 & 111 & & & & & & & & & & & & \\
\hline vWA & 111 & & & & & & & & & & & & \\
\hline TPOX & 111 & & & & & & & & & & & & \\
\hline D18S51 & 111 & & & & & & & & & & & & \\
\hline D5S818 & 111 & & & & & & & & & & & & \\
\hline FGA & 111 & & & & & & 0.0045 & & & & & 0.0135 & 0.0045 \\
\hline
\end{tabular}

\section{Supplementary Table 1: Allelic Frequencies for Abaco (Cont'd)}

\begin{tabular}{|c|c|c|c|c|c|c|c|c|c|c|c|c|c|c|}
\hline & & \multicolumn{13}{|c|}{ Allele } \\
\hline & $\mathbf{n}$ & 6 & 7 & 8 & 9 & 9.3 & 10 & 10.2 & 11 & 11.2 & 12 & 12.2 & 13 & 13.2 \\
\hline D8S1179 & 112 & & & & 0.0045 & & & & 0.0357 & & 0.0893 & & 0.1964 & \\
\hline D21S11 & 112 & & & & & & & & & & & & & \\
\hline D7S820 & 112 & & & 0.1786 & 0.1116 & & 0.3214 & & 0.2634 & & 0.1116 & & 0.0089 & \\
\hline CSF1PO & 112 & & 0.0268 & 0.0268 & 0.0357 & & 0.2991 & & 0.3080 & & 0.2723 & & 0.0268 & \\
\hline D3S1358 & 112 & & & & & & & & & & & & 0.0089 & \\
\hline TH01 & 112 & 0.1384 & 0.3348 & 0.2232 & 0.1250 & 0.1518 & 0.0268 & & & & & & & \\
\hline D13S317 & 112 & & & 0.0179 & 0.0134 & & 0.0223 & & 0.2500 & & 0.4286 & & 0.2054 & \\
\hline D16S539 & 112 & 0.0179 & & 0.0446 & 0.2321 & & 0.1920 & & 0.2500 & & 0.1384 & & 0.1161 & \\
\hline D2S1338 & 112 & & & & & & & & & & & & & \\
\hline D19S433 & 112 & & & & & & 0.0045 & 0.0045 & 0.0670 & & 0.0893 & 0.0536 & 0.2991 & 0.0268 \\
\hline vWA & 112 & & & & & & & & & & & & & \\
\hline TPOX & 111 & 0.0676 & 0.0586 & 0.3063 & 0.1667 & & 0.0676 & & 0.3018 & & 0.0315 & & & \\
\hline D18S51 & 112 & & & & 0.0045 & & 0.0134 & & 0.0089 & & 0.0670 & & 0.0625 & 0.0045 \\
\hline D5S818 & 112 & & & 0.0670 & 0.0223 & & 0.0670 & & 0.2500 & & 0.3661 & & 0.2232 & \\
\hline FGA & 112 & & & & & & & & & & & & & \\
\hline
\end{tabular}

\section{Supplementary Table 2: Allelic Frequencies for Eleuthera}




\begin{tabular}{|c|c|c|c|c|c|c|c|c|c|c|c|c|c|c|}
\cline { 2 - 14 } \multicolumn{1}{|c|}{} & $\mathbf{n}$ & $\mathbf{1 4}$ & $\mathbf{1 4 . 2}$ & $\mathbf{1 5}$ & $\mathbf{1 5 . 2}$ & $\mathbf{1 6}$ & $\mathbf{1 6 . 2}$ & $\mathbf{1 7}$ & $\mathbf{1 7 . 2}$ & $\mathbf{1 8}$ & $\mathbf{1 8 . 2}$ & $\mathbf{1 9}$ & $\mathbf{1 9 . 2}$ & $\mathbf{2 0}$ \\
\hline D8S1179 & 112 & 0.3750 & & 0.2277 & & 0.0670 & & 0.0045 & & & & & & \\
\hline D21S11 & 112 & & & & & & & & & & & & & \\
\hline D7S820 & 112 & 0.0045 & & & & & & & & & & & & \\
\hline CSF1PO & 112 & 0.0045 & & & & & & & & & & & & \\
\hline D3S1358 & 112 & 0.0982 & & 0.2902 & & 0.2991 & & 0.2009 & & 0.0982 & & 0.0045 & & \\
\hline TH01 & 112 & & & & & & & & & & & & & \\
\hline D13S317 & 112 & 0.0625 & & & & & & & & & & & & \\
\hline D16S539 & 112 & 0.0089 & & & & & & & & & & & & \\
\hline D2S1338 & 112 & & & & & 0.0536 & & 0.1205 & & 0.0491 & & 0.2009 & & 0.0893 \\
\hline D19S433 & 112 & 0.2143 & 0.0804 & 0.0536 & 0.0491 & 0.0134 & 0.0446 & & & & & & & \\
\hline vWA & 112 & 0.0446 & & 0.2009 & & 0.3036 & & 0.2411 & & 0.1295 & & 0.0580 & & 0.0045 \\
\hline TPOX & 111 & & & & & & & & & & & & & \\
\hline D18S51 & 112 & 0.0759 & & 0.1652 & & 0.1473 & & 0.1786 & & 0.1071 & & 0.0804 & & 0.0357 \\
\hline D5S818 & 112 & 0.0045 & & & & & & & & & & & & \\
\hline FGA & 112 & & & & & & & 0.0045 & & 0.0045 & 0.0089 & 0.0491 & 0.0045 & 0.0625 \\
\hline
\end{tabular}

\begin{tabular}{|c|c|c|c|c|c|c|c|c|c|c|c|c|c|c|}
\hline & & \multicolumn{13}{|c|}{ Allele } \\
\hline & $\mathrm{n}$ & 21 & 22 & 22.2 & 23 & 24 & 25 & 26 & 27 & 28 & 29 & 30 & 30.2 & 30.3 \\
\hline D8S1179 & 112 & & & & & & & & & & & & & \\
\hline D21S11 & 112 & & & & & & & & 0.0357 & 0.2500 & 0.2188 & 0.1607 & 0.0312 & 0.0089 \\
\hline D7S820 & 112 & & & & & & & & & & & & & \\
\hline CSF1PO & 112 & & & & & & & & & & & & & \\
\hline D3S1358 & 112 & & & & & & & & & & & & & \\
\hline TH01 & 112 & & & & & & & & & & & & & \\
\hline D13S317 & 112 & & & & & & & & & & & & & \\
\hline D16S539 & 112 & & & & & & & & & & & & & \\
\hline D2S1338 & 112 & 0.1295 & 0.1027 & & 0.1116 & 0.0938 & 0.0223 & 0.0223 & 0.0045 & & & & & \\
\hline D19S433 & 112 & & & & & & & & & & & & & \\
\hline vWA & 112 & 0.0134 & 0.0045 & & & & & & & & & & & \\
\hline TPOX & 111 & & & & & & & & & & & & & \\
\hline D18S51 & 112 & 0.0179 & 0.0268 & & 0.0045 & & & & & & & & & \\
\hline D5S818 & 112 & & & & & & & & & & & & & \\
\hline FGA & 112 & 0.1696 & 0.1607 & 0.0089 & 0.1429 & 0.1830 & 0.0804 & 0.0625 & 0.0268 & 0.0089 & 0.0134 & 0.0045 & & \\
\hline
\end{tabular}

\begin{tabular}{|c|c|c|c|c|c|c|c|c|c|c|c|}
\hline & & \multicolumn{10}{|c|}{ Allele } \\
\hline & $\mathrm{n}$ & 31 & 31.2 & 32 & 32.2 & 33 & 33.2 & 34 & 35 & 36 & 43.2 \\
\hline D8S1179 & 112 & & & & & & & & & & \\
\hline D21S11 & 112 & 0.1027 & 0.0223 & 0.0223 & 0.0357 & 0.0089 & 0.0357 & 0.0179 & 0.0402 & 0.0089 & \\
\hline D7S820 & 112 & & & & & & & & & & \\
\hline CSF1PO & 112 & & & & & & & & & & \\
\hline D3S1358 & 112 & & & & & & & & & & \\
\hline TH01 & 112 & & & & & & & & & & \\
\hline D13S317 & 112 & & & & & & & & & & \\
\hline D16S539 & 112 & & & & & & & & & & \\
\hline D2S1338 & 112 & & & & & & & & & & \\
\hline D19S433 & 112 & & & & & & & & & & \\
\hline vWA & 112 & & & & & & & & & & \\
\hline TPOX & 111 & & & & & & & & & & \\
\hline D18S51 & 112 & & & & & & & & & & \\
\hline D5S818 & 112 & & & & & & & & & & \\
\hline FGA & 112 & & & & & & & & & & 0.0045 \\
\hline
\end{tabular}

\section{Supplementary Table 2: Allelic Frequencies for Eleuthera (Cont'd)}




\begin{tabular}{|c|c|c|c|c|c|c|c|c|c|c|c|c|c|}
\hline & & \multicolumn{12}{|c|}{ Allele } \\
\hline & $\mathbf{n}$ & 6 & 7 & 8 & 9 & 9.3 & 10 & 11 & 12 & 12.2 & 13 & 13.2 & 14 \\
\hline D8S1179 & 92 & & & & & & 0.0109 & 0.0489 & 0.1087 & & 0.1848 & & 0.3859 \\
\hline D21S11 & 92 & & & & & & & & & & & & \\
\hline D7S820 & 92 & 0.0054 & 0.0163 & 0.2717 & 0.1250 & & 0.2935 & 0.1793 & 0.0815 & & 0.0217 & & 0.0054 \\
\hline CSF1PO & 92 & & 0.0435 & 0.0435 & 0.0272 & & 0.3098 & 0.2337 & 0.3043 & & 0.0380 & & \\
\hline D3S1358 & 92 & & & & & & & & 0.0109 & & & & 0.1087 \\
\hline TH01 & 92 & 0.1739 & 0.3587 & 0.2065 & 0.1630 & 0.0924 & 0.0054 & & & & & & \\
\hline D13S317 & 92 & & & 0.0109 & 0.0163 & & 0.0163 & 0.3261 & 0.4130 & & 0.1576 & & 0.0598 \\
\hline D16S539 & 92 & & & & 0.1359 & & 0.1739 & 0.3533 & 0.2174 & & 0.1033 & & 0.0163 \\
\hline D2S1338 & 92 & & & & & & & & & & & & \\
\hline D19S433 & 92 & & & & & & 0.0163 & 0.0761 & 0.1467 & 0.0380 & 0.3152 & 0.0435 & 0.2120 \\
\hline VWA & 92 & & & & & & & & 0.0109 & & 0.0163 & & 0.0707 \\
\hline TPOX & 92 & 0.0435 & 0.0435 & 0.2935 & 0.2228 & & 0.1304 & 0.2337 & 0.0326 & & & & \\
\hline D18S51 & 92 & & & & & & & 0.0109 & 0.0380 & & 0.0435 & 0.0163 & 0.0380 \\
\hline D5S818 & 92 & & 0.0054 & 0.1033 & 0.0109 & & 0.0598 & 0.1957 & 0.3098 & & 0.2772 & & 0.0272 \\
\hline FGA & 92 & & & & & & & & & & & & \\
\hline
\end{tabular}

\begin{tabular}{|c|c|c|c|c|c|c|c|c|c|c|c|c|c|}
\hline & & \multicolumn{12}{|c|}{ Allele } \\
\hline & $\mathbf{n}$ & 14.2 & 15 & 15.2 & 16 & 16.2 & 17 & 17.2 & 18 & 18.2 & 19 & 20 & 21 \\
\hline D8S1179 & 92 & & 0.2120 & & 0.0489 & & & & & & & & \\
\hline D21S11 & 92 & & & & & & & & & & & & \\
\hline D7S820 & 92 & & & & & & & & & & & & \\
\hline CSF1PO & 92 & & & & & & & & & & & & \\
\hline D3S1358 & 92 & & 0.3859 & & 0.3261 & & 0.1196 & & 0.0435 & & 0.0054 & & \\
\hline TH01 & 92 & & & & & & & & & & & & \\
\hline D13S317 & 92 & & & & & & & & & & & & \\
\hline D16S539 & 92 & & & & & & & & & & & & \\
\hline D2S1338 & 92 & & & & 0.0761 & & 0.0598 & & 0.0598 & & 0.1522 & 0.0598 & 0.1957 \\
\hline D19S433 & 92 & 0.0489 & 0.0326 & 0.0489 & & 0.0217 & & & & & & & \\
\hline vWA & 92 & & 0.2065 & & 0.2500 & & 0.2663 & & 0.0761 & & 0.0707 & 0.0217 & 0.0109 \\
\hline TPOX & 92 & & & & & & & & & & & & \\
\hline D18S51 & 92 & & 0.1957 & & 0.1576 & & 0.2065 & & 0.1141 & & 0.1141 & 0.0272 & 0.0217 \\
\hline D5S818 & 92 & & 0.0054 & & & & & & 0.0054 & & & & \\
\hline FGA & 92 & & & & & & & 0.0054 & 0.0054 & 0.0326 & 0.0598 & 0.0598 & 0.0870 \\
\hline
\end{tabular}

\begin{tabular}{|c|c|c|c|c|c|c|c|c|c|c|c|c|c|}
\hline & & \multicolumn{12}{|c|}{ Allele } \\
\hline & $\mathbf{n}$ & 21.2 & 22 & 22.2 & 23 & 23.2 & 24 & 25 & 26 & 27 & 28 & 29 & 30 \\
\hline D8S1179 & 92 & & & & & & & & & & & & \\
\hline D21S11 & 92 & & & & & & & & & 0.0326 & 0.1957 & 0.2065 & 0.2554 \\
\hline \begin{tabular}{|l} 
D7S820 \\
\end{tabular} & 92 & & & & & & & & & & & & \\
\hline CSF1PO & 92 & & & & & & & & & & & & \\
\hline D3S1358 & 92 & & & & & & & & & & & & \\
\hline TH01 & 92 & & & & & & & & & & & & \\
\hline D13S317 & 92 & & & & & & & & & & & & \\
\hline D16S539 & 92 & & & & & & & & & & & & \\
\hline D2S1338 & 92 & & 0.1359 & & 0.0707 & 0.0054 & 0.1196 & 0.0435 & 0.0217 & & & & \\
\hline D19S433 & 92 & & & & & & & & & & & & \\
\hline vWA & 92 & & & & & & & & & & & & \\
\hline TPOX & 92 & & & & & & & & & & & & \\
\hline D18S51 & 92 & 0.0054 & 0.0109 & & & & & & & & & & \\
\hline D5S818 & 92 & & & & & & & & & & & & \\
\hline FGA & 92 & 0.0054 & 0.1739 & 0.0054 & 0.1685 & & 0.1467 & 0.1087 & 0.0761 & 0.0380 & 0.0054 & 0.0054 & \\
\hline
\end{tabular}

\section{Supplementary Table 3: Allelic Frequencies for Exuma}




\begin{tabular}{|c|c|c|c|c|c|c|c|c|c|c|c|c|}
\cline { 2 - 14 } \multicolumn{1}{c|}{} & $\mathbf{n}$ & $\mathbf{3 0 . 2}$ & $\mathbf{3 1}$ & $\mathbf{3 1 . 2}$ & $\mathbf{3 2}$ & $\mathbf{3 2 . 2}$ & $\mathbf{3 3 . 1}$ & $\mathbf{3 3 . 2}$ & $\mathbf{3 5}$ & $\mathbf{3 7}$ & $\mathbf{4 3 . 2}$ & $\mathbf{4 4 . 2}$ \\
\hline D8S1179 & 92 & & & & & & & & & & & \\
\hline D21S11 & 92 & 0.0326 & 0.0707 & 0.0489 & 0.0109 & 0.0815 & 0.0054 & 0.0272 & 0.0272 & 0.0054 & & \\
\hline D7S820 & 92 & & & & & & & & & & & \\
\hline CSF1PO & 92 & & & & & & & & & & & \\
\hline D3S1358 & 92 & & & & & & & & & & & \\
\hline TH01 & 92 & & & & & & & & & & & \\
\hline D13S317 & 92 & & & & & & & & & & & \\
\hline D16S539 & 92 & & & & & & & & & & & \\
\hline D2S1338 & 92 & & & & & & & & & & & \\
\hline D19S433 & 92 & & & & & & & & & & & \\
\hline VWA & 92 & & & & & & & & & & & \\
\hline TPOX & 92 & & & & & & & & & & & \\
\hline D18S51 & 92 & & & & & & & & & & & \\
\hline D5S818 & 92 & & & & & & & & & & & \\
\hline FGA & 92 & & & & & & & & & & 0.0054 & 0.0109 \\
\hline
\end{tabular}

\section{Supplementary Table 3: Allelic Frequencies for Exuma (Cont'd)}

\begin{tabular}{|c|c|c|c|c|c|c|c|c|c|c|c|c|c|}
\cline { 2 - 2 } & \multicolumn{9}{|c|}{ Allele } \\
\hline & $\mathbf{n}$ & $\mathbf{6}$ & $\mathbf{7}$ & $\mathbf{8}$ & $\mathbf{9}$ & $\mathbf{9 . 3}$ & $\mathbf{1 0}$ & $\mathbf{1 0 . 2}$ & $\mathbf{1 1}$ & $\mathbf{1 2}$ & $\mathbf{1 2 . 2}$ & $\mathbf{1 3}$ & $\mathbf{1 3 . 2}$ \\
\hline D8S1179 & 133 & & & 0.0038 & & & 0.0113 & & 0.0677 & 0.1353 & & 0.1729 & \\
\hline D21S11 & 133 & & & & & & & & & & & & \\
\hline D7S820 & 133 & & 0.0075 & 0.2594 & 0.1053 & & 0.2707 & & 0.1842 & 0.1353 & & 0.0376 & \\
\hline CSF1PO & 133 & 0.0038 & 0.0602 & 0.0827 & 0.0376 & & 0.3045 & & 0.1955 & 0.2707 & & 0.0376 & \\
\hline D3S1358 & 133 & & & & & & & & & & & 0.0038 & \\
\hline TH01 & 133 & 0.1429 & 0.3534 & 0.2632 & 0.1353 & 0.0902 & 0.0150 & & & & & & \\
\hline D13S317 & 133 & & & 0.0414 & 0.0188 & & 0.0301 & & 0.2406 & 0.4135 & & 0.1992 & \\
\hline D16S539 & 133 & & & 0.0489 & 0.1917 & & 0.0940 & & 0.3271 & 0.1805 & & 0.1353 & \\
\hline D2S1338 & 133 & & & & & & & & & & & & \\
\hline D19S433 & 133 & & & & & & 0.0113 & & 0.0451 & 0.1241 & 0.0489 & 0.2368 & 0.0526 \\
\hline vWA & 133 & & & & & & & & 0.0113 & & & 0.0188 & \\
\hline TPOX & 133 & 0.0639 & 0.0301 & 0.3421 & 0.2444 & & 0.1165 & & 0.1767 & 0.0263 & & & \\
\hline D18S51 & 133 & & & & & & & 0.0038 & & 0.0489 & & 0.0714 & 0.0038 \\
\hline D5S818 & 132 & & & 0.0682 & 0.0114 & & 0.0492 & & 0.2273 & 0.2955 & & 0.3258 & \\
\hline FGA & 133 & & & & & & & & & & & & \\
\hline
\end{tabular}

\section{Supplementary Table 4: Allelic Frequencies for Grand Bahama}




\begin{tabular}{|c|c|c|c|c|c|c|c|c|c|c|c|c|c|}
\cline { 2 - 14 } \multicolumn{1}{c|}{} & \multicolumn{9}{|c|}{ Allele } \\
\hline & $\mathbf{n}$ & $\mathbf{1 4}$ & $\mathbf{1 4 . 1}$ & $\mathbf{1 4 . 2}$ & $\mathbf{1 5}$ & $\mathbf{1 5 . 2}$ & $\mathbf{1 6}$ & $\mathbf{1 6 . 2}$ & $\mathbf{1 7}$ & $\mathbf{1 7 . 2}$ & $\mathbf{1 8}$ & $\mathbf{1 8 . 2}$ & $\mathbf{1 9}$ \\
\hline D8S1179 & 133 & 0.3383 & & & 0.1805 & & 0.0789 & & 0.0075 & & 0.0038 & & \\
\hline D21S11 & 133 & & & & & & & & & & & & \\
\hline D7S820 & 133 & & & & & & & & & & & & \\
\hline CSF1PO & 133 & 0.0075 & & & & & & & & & & & \\
\hline D3S1358 & 133 & 0.1165 & & & 0.3684 & & 0.2707 & & 0.1955 & & 0.0451 & & \\
\hline TH01 & 133 & & & & & & & & & & & & \\
\hline D13S317 & 133 & 0.0564 & & & & & & & & & & & \\
\hline D16S539 & 133 & 0.0226 & & & & & & & & & & & \\
\hline D2S1338 & 133 & & & & 0.0038 & & 0.0526 & & 0.0977 & & 0.0451 & & 0.1842 \\
\hline D19S433 & 133 & 0.1842 & 0.0038 & 0.0940 & 0.0602 & 0.0639 & 0.0075 & 0.0526 & & 0.0150 & & & \\
\hline vWA & 133 & 0.0489 & & & 0.1654 & & 0.2820 & & 0.2143 & & 0.1278 & & 0.0865 \\
\hline TPOX & 133 & & & & & & & & & & & & \\
\hline D18S51 & 133 & 0.0564 & & & 0.1579 & 0.0075 & 0.2180 & & 0.1541 & & 0.1203 & & 0.0940 \\
\hline D5S818 & 132 & 0.0152 & & & 0.0076 & & & & & & & & \\
\hline FGA & 133 & & & & & & & & & 0.0075 & 0.0038 & 0.0038 & 0.0677 \\
\hline
\end{tabular}

\begin{tabular}{|c|c|c|c|c|c|c|c|c|c|c|c|c|c|}
\hline & & \multicolumn{12}{|c|}{ Allele } \\
\hline & $\mathrm{n}$ & 19.2 & 20 & 21 & 22 & 22.2 & 23 & 24 & 25 & 26 & 27 & 28 & 29 \\
\hline D8S1179 & 133 & & & & & & & & & & & & \\
\hline D21S11 & 133 & & & & & & & & & & 0.0639 & 0.2256 & 0.2068 \\
\hline D7S820 & 133 & & & & & & & & & & & & \\
\hline CSF1PO & 133 & & & & & & & & & & & & \\
\hline D3S1358 & 133 & & & & & & & & & & & & \\
\hline TH01 & 133 & & & & & & & & & & & & \\
\hline D13S317 & 133 & & & & & & & & & & & & \\
\hline D16S539 & 133 & & & & & & & & & & & & \\
\hline D2S1338 & 133 & & 0.0940 & 0.1165 & 0.1090 & & 0.0902 & 0.0940 & 0.0827 & 0.0301 & & & \\
\hline D19S433 & 133 & & & & & & & & & & & & \\
\hline vWA & 133 & & 0.0338 & 0.0038 & 0.0075 & & & & & & & & \\
\hline TPOX & 133 & & & & & & & & & & & & \\
\hline D18S51 & 133 & & 0.0414 & 0.0188 & 0.0038 & & & & & & & & \\
\hline D5S818 & 132 & & & & & & & & & & & & \\
\hline FGA & 133 & 0.0075 & 0.0301 & 0.0827 & 0.1955 & 0.0075 & 0.2256 & 0.1579 & 0.1278 & 0.0338 & 0.0301 & 0.0075 & 0.0038 \\
\hline
\end{tabular}

\begin{tabular}{|c|c|c|c|c|c|c|c|c|c|c|c|c|}
\cline { 2 - 13 } \multicolumn{1}{|c|}{} & $\mathbf{n}$ & $\mathbf{3 0}$ & $\mathbf{3 0 . 2}$ & $\mathbf{3 1}$ & $\mathbf{3 1 . 2}$ & $\mathbf{3 2}$ & $\mathbf{3 2 . 2}$ & $\mathbf{3 3}$ & $\mathbf{3 3 . 2}$ & $\mathbf{3 4}$ & $\mathbf{3 4 . 2}$ & $\mathbf{3 5}$ \\
\hline D8S1179 & 133 & & & & & & & & & & & \\
\hline D21S11 & 133 & 0.2218 & 0.0150 & 0.0564 & 0.0414 & 0.0075 & 0.0940 & 0.0075 & 0.0263 & 0.0075 & 0.0038 & 0.0226 \\
\hline D7S820 & 133 & & & & & & & & & & & \\
\hline CSF1PO & 133 & & & & & & & & & & & \\
\hline D3S1358 & 133 & & & & & & & & & & & \\
\hline TH01 & 133 & & & & & & & & & & & \\
\hline D13S317 & 133 & & & & & & & & & & & \\
\hline D16S539 & 133 & & & & & & & & & & & \\
\hline D2S1338 & 133 & & & & & & & & & & & \\
\hline D19S433 & 133 & & & & & & & & & & & \\
\hline vWA & 133 & & & & & & & & & & & \\
\hline TPOX & 133 & & & & & & & & & & & \\
\hline D18S51 & 133 & & & & & & & & & & & \\
\hline D5S818 & 132 & & & & & & & & & & & \\
\hline FGA & 133 & 0.0075 & & & & & & & & & & \\
\hline
\end{tabular}

\section{Supplementary Table 4: Allelic Frequencies for Grand Bahama (Cont'd)}




\begin{tabular}{|c|c|c|c|c|c|c|c|c|c|c|c|c|c|}
\hline & & \multicolumn{12}{|c|}{ Allele } \\
\hline & $\mathrm{n}$ & 6 & 7 & 8 & 9 & 9.3 & 10 & 11 & 11.1 & 12 & 12.2 & 13 & 13.2 \\
\hline D8S1179 & 87 & & & 0.0057 & 0.0172 & & 0.0402 & 0.0977 & & 0.1264 & & 0.1092 & \\
\hline D21S11 & 87 & & & & & & & & & & & & \\
\hline D7S820 & 87 & & 0.0057 & 0.1494 & 0.1379 & & 0.3448 & 0.2011 & 0.0057 & 0.0862 & & 0.0690 & \\
\hline CSF1PO & 87 & & 0.0345 & 0.0115 & 0.0115 & & 0.3448 & 0.2069 & & 0.2701 & & 0.1149 & \\
\hline D3S1358 & 87 & & & & & & & & & 0.0115 & & 0.0057 & \\
\hline TH01 & 87 & 0.1724 & 0.3103 & 0.2414 & 0.1782 & 0.0977 & & & & & & & \\
\hline D13S317 & 87 & & & 0.0862 & 0.0287 & & 0.0690 & 0.2816 & & 0.3391 & & 0.1207 & \\
\hline D16S539 & 87 & & & 0.0460 & 0.1379 & & 0.0920 & 0.3046 & & 0.2356 & & 0.1724 & \\
\hline D2S1338 & 87 & & & & & & & & & & & & \\
\hline D19S433 & 87 & & & & & & & 0.0747 & & 0.1034 & 0.0747 & 0.2414 & 0.0460 \\
\hline vWA & 87 & & & & & & & 0.0115 & & & & 0.0230 & \\
\hline TPOX & 87 & 0.0460 & 0.0287 & 0.3678 & 0.1897 & & 0.1034 & 0.2241 & & 0.0402 & & & \\
\hline D18S51 & 87 & & & & & & 0.0230 & 0.0057 & & 0.0517 & & 0.1379 & 0.0057 \\
\hline D5S818 & 87 & & & 0.0402 & & & 0.0575 & 0.2759 & & 0.3161 & & 0.3103 & \\
\hline FGA & 87 & & & & & & & & & & & & \\
\hline
\end{tabular}

\begin{tabular}{|c|c|c|c|c|c|c|c|c|c|c|c|c|c|}
\hline & & \multicolumn{12}{|c|}{ Allele } \\
\hline & $\mathrm{n}$ & 14 & 14.2 & 15 & 15.2 & 16 & 16.2 & 17 & 17.2 & 18 & 19 & 20 & 21 \\
\hline D8S1179 & 87 & 0.282 & & 0.2529 & & 0.035 & & 0.0287 & & 0.0057 & & & \\
\hline D21S11 & 87 & & & & & & & & & & & & \\
\hline D7S820 & 87 & & & & & & & & & & & & \\
\hline CSF1PO & 87 & & & 0.0057 & & & & & & & & & \\
\hline D3S1358 & 87 & 0.058 & & 0.2241 & & 0.402 & & 0.2356 & & 0.0632 & & & \\
\hline TH01 & 87 & & & & & & & & & & & & \\
\hline D13S317 & 87 & 0.0690 & & 0.0057 & & & & & & & & & \\
\hline D16S539 & 87 & 0.0115 & & & & & & & & & & & \\
\hline D2S1338 & 87 & & & & & 0.0287 & & 0.1437 & & 0.0460 & 0.1494 & 0.0632 & 0.0862 \\
\hline D19S433 & 87 & 0.1954 & 0.0230 & 0.0977 & 0.0805 & 0.0115 & 0.0345 & & 0.0172 & & & & \\
\hline vWA & 87 & 0.1149 & & 0.1782 & & 0.2184 & & 0.2241 & & 0.1897 & 0.0230 & 0.0172 & \\
\hline TPOX & 87 & & & & & & & & & & & & \\
\hline D18S51 & 87 & 0.1379 & & 0.1379 & 0.0057 & 0.0862 & & 0.1552 & & 0.1034 & 0.0690 & 0.0460 & 0.0172 \\
\hline D5S818 & 87 & & & & & & & & & & & & \\
\hline FGA & 87 & & & & & & & & & 0.0057 & 0.0862 & 0.1149 & 0.1092 \\
\hline
\end{tabular}

\begin{tabular}{|c|c|c|c|c|c|c|c|c|c|c|c|c|c|}
\hline & & \multicolumn{12}{|c|}{ Allele } \\
\hline & $\mathrm{n}$ & 21.2 & 22 & 22.2 & 23 & 24 & 25 & 26 & 27 & 28 & 29 & 30 & 30.2 \\
\hline D8S1179 & 87 & & & & & & & & & & & & \\
\hline D21S11 & 87 & & & & & & & & 0.0345 & 0.2414 & 0.1149 & 0.2931 & 0.0402 \\
\hline D7S820 & 87 & & & & & & & & & & & & \\
\hline CSF1PO & 87 & & & & & & & & & & & & \\
\hline D3S1358 & 87 & & & & & & & & & & & & \\
\hline ТH01 & 87 & & & & & & & & & & & & \\
\hline D13S317 & 87 & & & & & & & & & & & & \\
\hline D16S539 & 87 & & & & & & & & & & & & \\
\hline D2S1338 & 87 & & 0.1149 & & 0.1782 & 0.0517 & 0.0920 & 0.0460 & & & & & \\
\hline D19S433 & 87 & & & & & & & & & & & & \\
\hline vWA & 87 & & & & & & & & & & & & \\
\hline TPOX & 87 & & & & & & & & & & & & \\
\hline D18S51 & 87 & 0.0057 & 0.0057 & & 0.0057 & & & & & & & & \\
\hline D5S818 & 87 & & & & & & & & & & & & \\
\hline FGA & 87 & & 0.1667 & 0.0287 & 0.1437 & 0.1264 & 0.0862 & 0.0172 & 0.0172 & 0.0287 & & 0.0057 & \\
\hline
\end{tabular}

\section{Supplementary Table 5: Allelic Frequencies for Long Island}




\begin{tabular}{|c|c|c|c|c|c|c|c|c|c|c|c|c|}
\cline { 2 - 14 } \\
\cline { 2 - 14 } & n & $\mathbf{3 1}$ & $\mathbf{3 1 . 2}$ & $\mathbf{3 2}$ & $\mathbf{3 2 . 2}$ & $\mathbf{3 3}$ & $\mathbf{3 3 . 2}$ & $\mathbf{3 4}$ & $\mathbf{3 5}$ & $\mathbf{3 6}$ & $\mathbf{4 3 . 2}$ & $\mathbf{4 4 . 2}$ \\
\hline D8S1179 & 87 & & & & & & & & & & & \\
\hline D21S11 & 87 & 0.0805 & 0.0345 & 0.0115 & 0.0747 & 0.0057 & 0.0172 & 0.0057 & 0.0287 & 0.0172 & & \\
\hline D7S820 & 87 & & & & & & & & & & & \\
\hline CSF1PO & 87 & & & & & & & & & & & \\
\hline D3S1358 & 87 & & & & & & & & & & & \\
\hline TH01 & 87 & & & & & & & & & & & \\
\hline D13S317 & 87 & & & & & & & & & & & \\
\hline D16S539 & 87 & & & & & & & & & & & \\
\hline D2S1338 & 87 & & & & & & & & & & & \\
\hline D19S433 & 87 & & & & & & & & & & & \\
\hline vWA & 87 & & & & & & & & & & & \\
\hline TPOX & 87 & & & & & & & & & & & \\
\hline D18S51 & 87 & & & & & & & & & & & \\
\hline D5S818 & 87 & & & & & & & & & & & \\
\hline FGA & 87 & & 0.0172 & & & & & & & & 0.0057 & 0.0402 \\
\hline
\end{tabular}

Supplementary Table 5: Allelic Frequencies for Long Island (Cont'd) 


\begin{tabular}{|c|c|c|c|c|c|c|}
\hline \multirow[b]{2}{*}{ Locus } & \multicolumn{6}{|c|}{ Abaco } \\
\hline & GDI & MP & PD & PIC & PE & TPI \\
\hline D8S1179 & 0.81301 & 0.06176 & 0.93824 & 0.78341 & 0.53712 & 2.13 \\
\hline D21S11 & 0.86776 & 0.04099 & 0.95901 & 0.84942 & 0.81571 & 5.55 \\
\hline D7S820 & 0.79475 & 0.08027 & 0.91973 & 0.76087 & 0.67104 & 3.08 \\
\hline CSF1PO & 0.77690 & 0.09796 & 0.90204 & 0.73847 & 0.65359 & 2.92 \\
\hline D3S1358 & 0.75692 & 0.11436 & 0.88564 & 0.71195 & 0.52140 & 2.06 \\
\hline TH01 & 0.76870 & 0.09926 & 0.90074 & 0.73016 & 0.56930 & 2.31 \\
\hline D13S317 & 0.75541 & 0.11257 & 0.88743 & 0.71525 & 0.56930 & 2.31 \\
\hline D16S539 & 0.80996 & 0.06728 & 0.93272 & 0.77783 & 0.49070 & 1.91 \\
\hline D2S1338 & 0.89434 & 0.03222 & 0.96778 & 0.88010 & 0.72436 & 3.70 \\
\hline D19S433 & 0.84379 & 0.04342 & 0.95658 & 0.82360 & 0.70644 & 3.47 \\
\hline vWA & 0.82165 & 0.06323 & 0.93677 & 0.79379 & 0.67104 & 3.08 \\
\hline TPOX & 0.77869 & 0.08855 & 0.91145 & 0.74252 & 0.55309 & 2.22 \\
\hline D18S51 & 0.88158 & 0.03222 & 0.96778 & 0.86561 & 0.76060 & 4.27 \\
\hline D5S818 & 0.76503 & 0.09569 & 0.90431 & 0.72474 & 0.52140 & 2.06 \\
\hline FGA & 0.86727 & 0.03969 & 0.96031 & 0.84921 & 0.67104 & 3.08 \\
\hline
\end{tabular}

\begin{tabular}{cccccc}
\hline \multicolumn{6}{c}{ Eleuthera } \\
\hline GDI & MP & PD & PIC & PE & TPI \\
\hline 0.75857 & 0.09853 & 0.90147 & 0.71928 & 0.55669 & 2.24 \\
0.84910 & 0.05931 & 0.94069 & 0.82836 & 0.78085 & 4.67 \\
0.77386 & 0.08721 & 0.91279 & 0.73451 & 0.54081 & 2.15 \\
0.74135 & 0.11767 & 0.88233 & 0.69193 & 0.49461 & 1.93 \\
0.77002 & 0.11049 & 0.88951 & 0.72901 & 0.60569 & 2.55 \\
0.78303 & 0.08769 & 0.91231 & 0.74712 & 0.58914 & 2.43 \\
0.70992 & 0.13600 & 0.86400 & 0.66019 & 0.45071 & 1.75 \\
0.81538 & 0.06234 & 0.93766 & 0.78502 & 0.54081 & 2.15 \\
0.88625 & 0.03109 & 0.96891 & 0.87098 & 0.74469 & 4.00 \\
0.83837 & 0.05580 & 0.94420 & 0.81754 & 0.62244 & 2.67 \\
0.79056 & 0.08498 & 0.91502 & 0.75547 & 0.62244 & 2.67 \\
0.77726 & 0.09163 & 0.90837 & 0.74050 & 0.52140 & 2.06 \\
0.88837 & 0.03412 & 0.96588 & 0.87352 & 0.78085 & 4.67 \\
0.74752 & 0.11862 & 0.88138 & 0.70312 & 0.57280 & 2.33 \\
0.87748 & 0.03332 & 0.96668 & 0.86069 & 0.60569 & 2.55 \\
\hline
\end{tabular}

\begin{tabular}{cccccc}
\hline \multicolumn{6}{c}{ Exuma } \\
\hline GDI & MP & PD & PIC & PE & TPI \\
\hline 0.75944 & 0.10681 & 0.89319 & 0.72095 & 0.47332 & 1.84 \\
0.84058 & 0.04891 & 0.95109 & 0.81674 & 0.69058 & 3.29 \\
0.78908 & 0.09310 & 0.90690 & 0.75268 & 0.66933 & 3.07 \\
0.75493 & 0.11484 & 0.88516 & 0.70952 & 0.60713 & 2.56 \\
0.72054 & 0.13705 & 0.86295 & 0.67015 & 0.49135 & 1.92 \\
0.76746 & 0.09617 & 0.90383 & 0.72733 & 0.49135 & 1.92 \\
0.69779 & 0.13729 & 0.86271 & 0.64140 & 0.38907 & 1.53 \\
0.77251 & 0.08932 & 0.91068 & 0.73381 & 0.35821 & 1.44 \\
0.88673 & 0.03214 & 0.96786 & 0.87061 & 0.71207 & 3.54 \\
0.82294 & 0.06191 & 0.93809 & 0.79792 & 0.71207 & 3.54 \\
0.81159 & 0.06616 & 0.93384 & 0.78084 & 0.43842 & 1.70 \\
0.79205 & 0.09074 & 0.90926 & 0.75586 & 0.69058 & 3.29 \\
0.86636 & 0.04182 & 0.95818 & 0.84676 & 0.71207 & 3.54 \\
0.77798 & 0.09806 & 0.90194 & 0.73926 & 0.54768 & 2.19 \\
0.88952 & 0.03119 & 0.96881 & 0.87383 & 0.88926 & 9.20 \\
\hline
\end{tabular}

Supplementary Table 6: Forensic parameters for Abaco, Eleuthera, Exuma, Grand Bahama and Long Island across the 15 Identifiler loci 


\begin{tabular}{|c|c|c|c|c|c|c|}
\hline \multirow[b]{2}{*}{ Locus } & \multicolumn{6}{|c|}{ Grand Bahama } \\
\hline & GDI & MP & PD & PIC & PE & TPI \\
\hline D8S1179 & 0.79671 & 0.07298 & 0.92702 & 0.76661 & 0.60744 & 2.56 \\
\hline D21S11 & 0.84091 & 0.04980 & 0.95020 & 0.81837 & 0.69412 & 3.33 \\
\hline D7S820 & 0.79765 & 0.08305 & 0.91695 & 0.76398 & 0.60744 & 2.56 \\
\hline CSF1PO & 0.78539 & 0.08361 & 0.91639 & 0.75059 & 0.55249 & 2.22 \\
\hline D3S1358 & 0.73994 & 0.11267 & 0.88733 & 0.69351 & 0.45071 & 1.75 \\
\hline ТH01 & 0.76164 & 0.10295 & 0.89705 & 0.72180 & 0.60744 & 2.56 \\
\hline D13S317 & 0.72799 & 0.11369 & 0.88631 & 0.68395 & 0.37147 & 1.48 \\
\hline D16S539 & 0.79665 & 0.07321 & 0.92679 & 0.76535 & 0.60744 & 2.56 \\
\hline D2S1338 & 0.89604 & 0.02697 & 0.97303 & 0.88300 & 0.78468 & 4.75 \\
\hline D19S433 & 0.87093 & 0.03986 & 0.96014 & 0.85481 & 0.72396 & 3.69 \\
\hline vWA & 0.82242 & 0.06676 & 0.93324 & 0.79619 & 0.69412 & 3.33 \\
\hline TPOX & 0.77568 & 0.08915 & 0.91085 & 0.73957 & 0.56599 & 2.29 \\
\hline D18S51 & 0.87090 & 0.03533 & 0.96467 & 0.85389 & 0.62156 & 2.66 \\
\hline D5S818 & 0.75029 & 0.10227 & 0.89773 & 0.70524 & 0.49689 & 1.94 \\
\hline FGA & 0.85816 & 0.04438 & 0.95562 & 0.83890 & 0.72396 & 3.69 \\
\hline
\end{tabular}

\begin{tabular}{cccccc}
\hline \multicolumn{7}{c}{ Long Island } \\
\hline GDI & MP & PD & PIC & PE & TPI \\
\hline 0.82001 & 0.06355 & 0.93645 & 0.79206 & 0.44809 & 1.74 \\
0.82971 & 0.05351 & 0.94649 & 0.80533 & 0.56537 & 2.29 \\
0.79158 & 0.08257 & 0.91743 & 0.75838 & 0.69600 & 3.35 \\
0.75497 & 0.11560 & 0.88440 & 0.70991 & 0.50486 & 1.98 \\
0.72912 & 0.12010 & 0.87990 & 0.68109 & 0.30206 & 1.28 \\
0.77889 & 0.09711 & 0.90289 & 0.73817 & 0.50486 & 1.98 \\
0.77782 & 0.08786 & 0.91214 & 0.74180 & 0.54480 & 2.18 \\
0.79682 & 0.08416 & 0.91584 & 0.76209 & 0.67346 & 3.11 \\
0.88958 & 0.02946 & 0.97054 & 0.87338 & 0.65120 & 2.90 \\
0.86639 & 0.04664 & 0.95336 & 0.84737 & 0.67346 & 3.11 \\
0.82440 & 0.06355 & 0.93645 & 0.79441 & 0.58630 & 2.42 \\
0.76766 & 0.09658 & 0.90342 & 0.72943 & 0.52463 & 2.07 \\
0.89529 & 0.03025 & 0.96975 & 0.87995 & 0.69600 & 3.35 \\
0.72693 & 0.13251 & 0.86749 & 0.67095 & 0.44809 & 1.74 \\
0.89649 & 0.03290 & 0.96710 & 0.88141 & 0.74180 & 3.95 \\
\hline
\end{tabular}

Supplementary Table 6: Forensic parameters for Abaco, Eleuthera, Exuma, Grand Bahama and Long Island across the 15 Identifiler loci (Cont'd) 


\begin{tabular}{|c|c|c|c|c|c|c|c|c|c|c|}
\hline & $\mathbf{A B A}$ & ELU & EXU & GRB & LNG & NWP & HAT & JAM & US1 & US2 \\
\hline ABA & & 255.5159 & 257.4368 & 208.0772 & 262.4739 & 217.1170 & 246.8775 & 208.3699 & 212.2777 & 258.4031 \\
\hline ELU & 0.0004 & & 232.3493 & 255.7313 & 341.4149 & 248.6331 & 249.9456 & 222.2641 & 224.9921 & 248.9695 \\
\hline EXU & 0.0002 & 0.0038 & & 201.0366 & 342.4225 & 178.6515 & 236.5814 & 239.4758 & 239.6547 & 236.6326 \\
\hline GRB & 0.1061 & 0.0001 & 0.1301 & & 311.0807 & 199.8735 & 216.8389 & 190.3541 & 221.6427 & 210.3668 \\
\hline LNG & 0.0000 & 0.0000 & 0.0000 & 0.0000 & & 332.3794 & 341.7630 & 315.0483 & 317.1819 & 369.1798 \\
\hline NWP & 0.2210 & 0.0079 & 0.8231 & 0.4211 & 0.0000 & & 236.7342 & 228.4719 & 211.5335 & 260.9949 \\
\hline HAT & 0.0073 & 0.0027 & 0.0246 & 0.1006 & 0.0000 & 0.0851 & & 230.7371 & 232.8402 & 227.0359 \\
\hline JAM & 0.1668 & 0.0327 & 0.0043 & 0.4090 & 0.0000 & 0.1246 & 0.0454 & & 186.0889 & 216.0961 \\
\hline US1 & 0.1884 & 0.0357 & 0.0041 & 0.0292 & 0.0000 & 0.3462 & 0.0441 & 0.7311 & & 231.3660 \\
\hline US2 & 0.0016 & 0.0014 & 0.0080 & 0.1525 & 0.0000 & 0.0134 & 0.0887 & 0.1879 & 0.0516 & \\
\hline ANG & 0.0000 & 0.0000 & 0.0053 & 0.0001 & 0.0000 & 0.0000 & 0.0129 & 0.0003 & 0.0001 & 0.0000 \\
\hline GUB & 0.0000 & 0.0001 & 0.0743 & 0.0183 & 0.0000 & 0.0007 & 0.0106 & 0.0007 & 0.0000 & 0.0000 \\
\hline EGU & 0.0186 & 0.0000 & 0.0125 & 0.0670 & 0.0000 & 0.0428 & 0.9483 & 0.0122 & 0.0312 & 0.0112 \\
\hline NAM & 0.0000 & 0.0000 & 0.0000 & 0.0000 & 0.0000 & 0.0000 & 0.0000 & 0.0000 & 0.0000 & 0.0000 \\
\hline NIG & 0.0000 & 0.0000 & 0.0000 & 0.0000 & 0.0000 & 0.0000 & 0.0000 & 0.0000 & 0.0000 & 0.0000 \\
\hline HUT & 0.0000 & 0.0000 & 0.0000 & 0.0000 & 0.0000 & 0.0000 & 0.0000 & 0.0000 & 0.0000 & 0.0000 \\
\hline KEN & 0.0363 & 0.0289 & 0.0541 & 0.1101 & 0.0000 & 0.0749 & 0.2962 & 0.2032 & 0.1046 & 0.0246 \\
\hline MAD & 0.0000 & 0.0000 & 0.0000 & 0.0000 & 0.0000 & 0.0000 & 0.0000 & 0.0000 & 0.0000 & 0.0000 \\
\hline MOZ & 0.0000 & 0.0000 & 0.0000 & 0.0000 & 0.0000 & 0.0000 & 0.0000 & 0.0000 & 0.0000 & 0.0000 \\
\hline TAN & 0.0000 & 0.0000 & 0.0000 & 0.0000 & 0.0000 & 0.0000 & 0.0000 & 0.0000 & 0.0000 & 0.0000 \\
\hline TUT & 0.0000 & 0.0000 & 0.0000 & 0.0000 & 0.0000 & 0.0000 & 0.0000 & 0.0000 & 0.0000 & 0.0000 \\
\hline UGA & 0.0000 & 0.0000 & 0.0000 & 0.0000 & 0.0000 & 0.0000 & 0.0000 & 0.0000 & 0.0000 & 0.0000 \\
\hline SAF & 0.0000 & 0.0000 & 0.0000 & 0.0000 & 0.0000 & 0.0000 & 0.0000 & 0.0000 & 0.0000 & 0.0000 \\
\hline $\mathrm{CCS}$ & 0.0000 & 0.0000 & 0.0000 & 0.0000 & 0.0000 & 0.0000 & 0.0000 & 0.0000 & 0.0000 & 0.0000 \\
\hline JAP & 0.0000 & 0.0000 & 0.0000 & 0.0000 & 0.0000 & 0.0000 & 0.0000 & 0.0000 & 0.0000 & 0.0000 \\
\hline KOR & 0.0000 & 0.0000 & 0.0000 & 0.0000 & 0.0000 & 0.0000 & 0.0000 & 0.0000 & 0.0000 & 0.0000 \\
\hline MAL & 0.0000 & 0.0000 & 0.0000 & 0.0000 & 0.0000 & 0.0000 & 0.0000 & 0.0000 & 0.0000 & 0.0000 \\
\hline PHI & 0.0000 & 0.0000 & 0.0000 & 0.0000 & 0.0000 & 0.0000 & 0.0000 & 0.0000 & 0.0000 & 0.0000 \\
\hline CSH & 0.0000 & 0.0000 & 0.0000 & 0.0000 & 0.0000 & 0.0000 & 0.0000 & 0.0000 & 0.0000 & 0.0000 \\
\hline GUA & 0.0000 & 0.0000 & 0.0000 & 0.0000 & 0.0000 & 0.0000 & 0.0000 & 0.0000 & 0.0000 & 0.0000 \\
\hline KAK & 0.0000 & 0.0000 & 0.0000 & 0.0000 & 0.0000 & 0.0000 & 0.0000 & 0.0000 & 0.0000 & 0.0000 \\
\hline KIC & 0.0000 & 0.0000 & 0.0000 & 0.0000 & 0.0000 & 0.0000 & 0.0000 & 0.0000 & 0.0000 & 0.0000 \\
\hline YUC & 0.0000 & 0.0000 & 0.0000 & 0.0000 & 0.0000 & 0.0000 & 0.0000 & 0.0000 & 0.0000 & 0.0000 \\
\hline CAR & 0.0000 & 0.0000 & 0.0000 & 0.0000 & 0.0000 & 0.0000 & 0.0000 & 0.0000 & 0.0000 & 0.0000 \\
\hline MEX & 0.0000 & 0.0000 & 0.0000 & 0.0000 & 0.0000 & 0.0000 & 0.0000 & 0.0000 & 0.0000 & 0.0000 \\
\hline CSR & 0.0000 & 0.0000 & 0.0000 & 0.0000 & 0.0000 & 0.0000 & 0.0000 & 0.0000 & 0.0000 & 0.0000 \\
\hline ELS & 0.0000 & 0.0000 & 0.0000 & 0.0000 & 0.0000 & 0.0000 & 0.0000 & 0.0000 & 0.0000 & 0.0000 \\
\hline HIS & 0.0000 & 0.0000 & 0.0000 & 0.0000 & 0.0000 & 0.0000 & 0.0000 & 0.0000 & 0.0000 & 0.0000 \\
\hline HON & 0.0000 & 0.0000 & 0.0000 & 0.0000 & 0.0000 & 0.0000 & 0.0000 & 0.0000 & 0.0000 & 0.0000 \\
\hline MAR & 0.0000 & 0.0000 & 0.0000 & 0.0000 & 0.0000 & 0.0000 & 0.0000 & 0.0000 & 0.0000 & 0.0000 \\
\hline MET & 0.0000 & 0.0000 & 0.0000 & 0.0000 & 0.0000 & 0.0000 & 0.0000 & 0.0000 & 0.0000 & 0.0000 \\
\hline PRA & 0.0000 & 0.0000 & 0.0000 & 0.0000 & 0.0000 & 0.0000 & 0.0000 & 0.0000 & 0.0000 & 0.0000 \\
\hline BRA & 0.0000 & 0.0000 & 0.0000 & 0.0000 & 0.0000 & 0.0000 & 0.0000 & 0.0000 & 0.0000 & 0.0000 \\
\hline CAU & 0.0000 & 0.0000 & 0.0000 & 0.0000 & 0.0000 & 0.0000 & 0.0000 & 0.0000 & 0.0000 & 0.0000 \\
\hline GRE & 0.0000 & 0.0000 & 0.0000 & 0.0000 & 0.0000 & 0.0000 & 0.0000 & 0.0000 & 0.0000 & 0.0000 \\
\hline POR & 0.0000 & 0.0000 & 0.0000 & 0.0000 & 0.0000 & 0.0000 & 0.0000 & 0.0000 & 0.0000 & 0.0000 \\
\hline SPN & 0.0000 & 0.0000 & 0.0000 & 0.0000 & 0.0000 & 0.0000 & 0.0000 & 0.0000 & 0.0000 & 0.0000 \\
\hline SWE & 0.0000 & 0.0000 & 0.0000 & 0.0000 & 0.0000 & 0.0000 & 0.0000 & 0.0000 & 0.0000 & 0.0000 \\
\hline
\end{tabular}

Refer to Table 1 for key to abbreviations

Pair-wise population comparisons yielding statistically insignificant differences both before (in bold) and after (in italics) applying the Bonferroni correction

Supplementary Table 7: G-test 


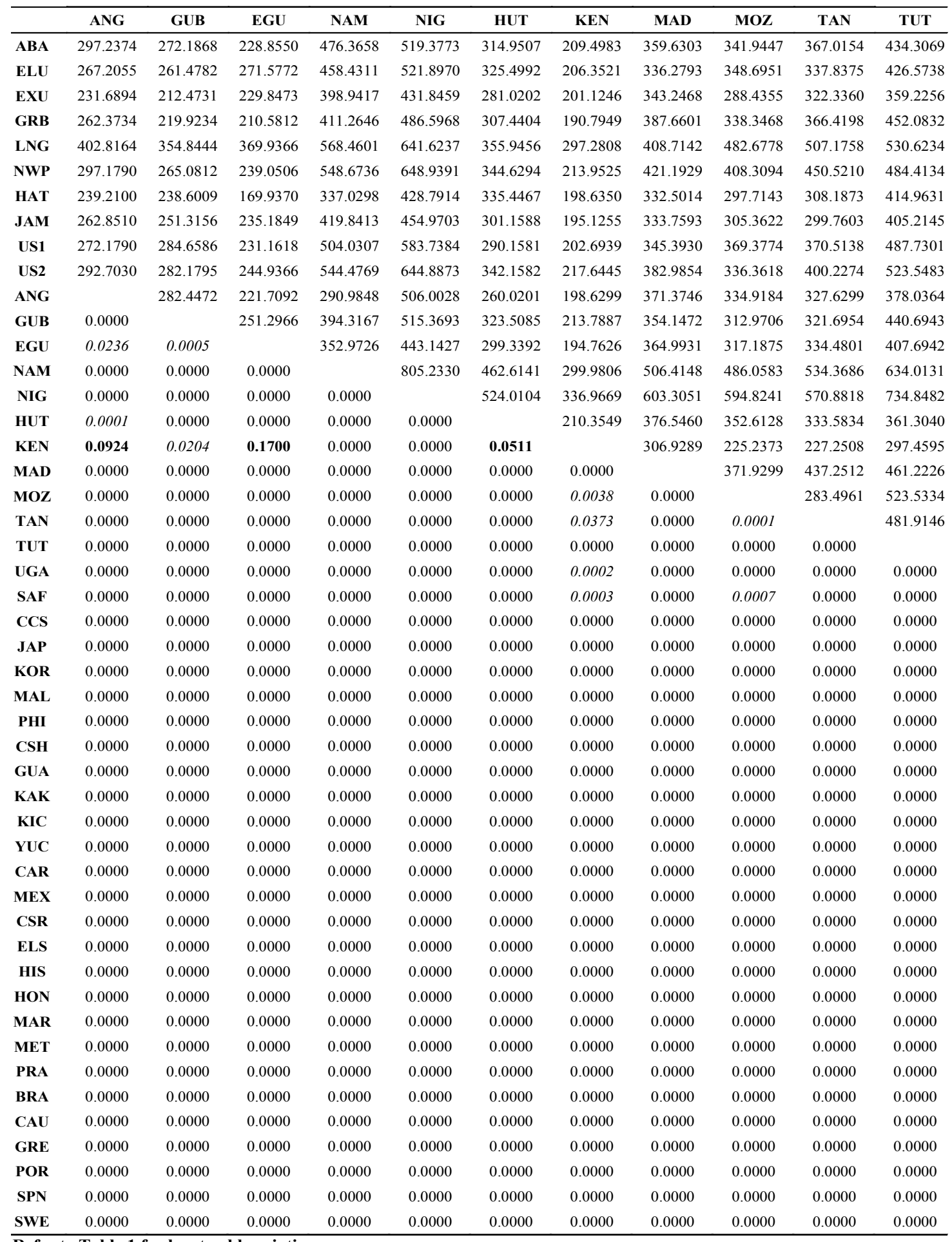

Refer to Table 1 for key to abbreviations

Pair-wise population comparisons yielding statistically insignificant differences both before (in bold) and after (in italics) applying the Bonferroni correction

Supplementary Table 7: G-test (Cont'd) 


\begin{tabular}{|c|c|c|c|c|c|c|c|c|c|c|c|}
\hline & UGA & SAF & CCS & JAP & KOR & MAL & PHI & CSH & GUA & KAK & KIC \\
\hline ABA & 353.1068 & 385.4726 & 1124.9126 & 1474.3500 & 1283.3197 & 967.7285 & 845.1508 & 1136.1504 & 1162.8175 & 1063.3929 & 618.9303 \\
\hline ELU & 466.6238 & 341.0126 & 1198.8967 & 1622.3893 & 1408.1648 & 1087.0167 & 935.8827 & 1256.2819 & 1116.3730 & 1051.5525 & 626.4266 \\
\hline EXU & 408.4935 & 307.4785 & 1160.9515 & 1484.1227 & 1334.0588 & 1045.5013 & 947.4545 & 1203.3729 & 1105.9020 & 1015.7192 & 632.3660 \\
\hline GRB & 380.2610 & 387.3344 & 1303.5280 & 1845.0591 & 1572.8790 & 1202.7966 & 1009.8267 & 1304.8824 & 1219.4445 & 1144.1353 & 668.3839 \\
\hline LNG & 435.1848 & 450.6429 & 949.6135 & 1221.7439 & 1070.1912 & 838.0112 & 788.5061 & 956.1392 & 1037.1217 & 976.2270 & 593.6605 \\
\hline NWP & 462.1212 & 414.8787 & 1640.0826 & 2567.4902 & 2044.1027 & 1505.4727 & 1227.9337 & 1753.9027 & 1347.5922 & 1277.1736 & 706.2735 \\
\hline HAT & 416.9154 & 279.4336 & 1258.7485 & 1775.1469 & 1540.2103 & 1153.0020 & 987.1977 & 1291.3613 & 1198.3019 & 1157.5844 & 689.5162 \\
\hline JAM & 367.2077 & 348.8613 & 1105.7494 & 1517.8855 & 1325.4003 & 987.2772 & 861.8778 & 1130.8444 & 1116.5532 & 1048.3948 & 613.2034 \\
\hline US1 & 436.9200 & 396.6248 & 1321.3734 & 1913.9091 & 1571.8273 & 1145.4186 & 956.6950 & 1366.2150 & 1197.0146 & 1140.5813 & 652.3872 \\
\hline US2 & 483.2510 & 399.4874 & 1612.5165 & 2607.3901 & 1978.6023 & 1474.4789 & 1150.3481 & 1668.1620 & 1335.1168 & 1301.6584 & 686.3676 \\
\hline ANG & 468.1497 & 310.2818 & 1319.5687 & 1819.9849 & 1547.0536 & 1230.0575 & 994.3469 & 1340.3058 & 1246.9574 & 1221.3892 & 736.8767 \\
\hline GUB & 416.2239 & 326.4199 & 1101.0726 & 1473.7946 & 1327.3401 & 1044.8998 & 898.3178 & 1120.6151 & 1073.9020 & 1037.8618 & 660.8270 \\
\hline EGU & 456.9542 & 333.283 & 1410.5985 & 1999.6361 & 1703.4598 & 1264.7218 & 1045.0815 & 1449.3634 & 1274.7736 & 1241.0842 & 709.8235 \\
\hline NAM & 622.8959 & 419.0391 & 1964.9546 & 3091.0125 & 2514.9470 & 1966.7240 & 1467.2649 & 2218.7803 & 1575.6923 & 1673.7672 & 947.6314 \\
\hline NIG & 716.3812 & 543.8169 & 2314.3889 & 3950.7646 & 2972.3582 & 2351.0574 & 1680.2054 & 2583.8701 & 1642.0138, & 1667.4712 & 892.8112 \\
\hline HUT & 328.6649 & 355.1657 & 1243.8484 & 1652.9045 & 1444.2677 & 1138.2894 & 1010.2482 & 1298.2859 & 1239.0825 & 1115.5770 & 692.9352 \\
\hline KEN & 241.7360 & 243.6538 & 965.3720 & 1168.9825 & 1112.2170 & 886.5229 & 784.0193 & 959.9223 & 997.4241 & 886.2337 & 580.9343 \\
\hline MAD & 501.2495 & 385.7041 & 708.5034 & 896.1970 & 857.8117 & 625.9515 & 527.1189 & 775.9848 & 843.7770 & 788.8383 & 538.3190 \\
\hline MOZ & 519.5912 & 246.7927 & 1516.899 & 2071.0671 & 1779.2944 & 1456.1948 & 1166.9080 & 1575.8204 & 1271.2258 & 1225.5809 & 712.3813 \\
\hline TAN & 527.5989 & 330.5381 & 1851.1737 & 2849.9404 & 2241.6169 & 1783.1946 & 1353.2587 & 1953.3273 & 1484.1809 & 1426.6024 & 787.9786 \\
\hline TUT & 537.8627 & 440.1677 & 1533.4893 & 2063.9529 & 1805.7122 & 1471.2946 & 1165.1117 & 1633.0477 & 1364.9073 & 1238.0286 & 725.4368 \\
\hline UGA & & 510.8528 & 1159.6693 & 1520.1287 & 1357.8638 & 1083.6028 & 974.2226 & 1209.6449 & 1242.2509 & 1069.8838 & 638.9430 \\
\hline SAF & 0.0000 & & 1347.2368 & 1861.0095 & 1613.6172 & 1294.1763 & 1088.1547 & 1417.8231 & 1237.6667 & 1186.8896 & 740.8685 \\
\hline $\mathrm{CCS}$ & 0.0000 & 0.0000 & & 450.1981 & 353.6910 & 432.0402 & 356.7864 & 279.7270 & 1063.6783 & 960.7687 & 561.3939 \\
\hline JAP & 0.0000 & 0.0000 & 0.0000 & & 307.2385 & 789.6036 & 512.5523 & 518.3922 & 1140.1155 & 1072.4084 & 554.2070 \\
\hline KOR & 0.0000 & 0.0000 & 0.0000 & 0.0000 & & 636.6891 & 459.4520 & 344.5786 & 1043.5566 & 962.5594 & 530.4380 \\
\hline MAL & 0.0000 & 0.0000 & 0.0000 & 0.0000 & 0.0000 & & 289.0813 & 472.2081 & 1056.2954 & 934.7960 & 539.4123 \\
\hline PHI & 0.0000 & 0.0000 & 0.0000 & 0.0000 & 0.0000 & 0.0000 & & 377.1124 & 948.9913 & 929.8623 & 573.2806 \\
\hline CSH & 0.0000 & 0.0000 & 0.0000 & 0.0000 & 0.0000 & 0.0000 & 0.0000 & & 1043.9146 & 915.2687 & 517.5212 \\
\hline GUA & 0.0000 & 0.0000 & 0.0000 & 0.0000 & 0.0000 & 0.0000 & 0.0000 & 0.0000 & & 435.1290 & 342.1435 \\
\hline KAK & 0.0000 & 0.0000 & 0.0000 & 0.0000 & 0.0000 & 0.0000 & 0.0000 & 0.0000 & 0.0000 & & 117.3686 \\
\hline KIC & 0.0000 & 0.0000 & 0.0000 & 0.0000 & 0.0000 & 0.0000 & 0.0000 & 0.0000 & 0.0000 & 0.7793 & \\
\hline YUC & 0.0000 & 0.0000 & 0.0000 & 0.0000 & 0.0000 & 0.0000 & 0.0000 & 0.0000 & 0.0000 & 0.0418 & 0.3783 \\
\hline CAR & 0.0000 & 0.0000 & 0.0000 & 0.0000 & 0.0000 & 0.0000 & 0.0000 & 0.0000 & 0.0000 & 0.0000 & 0.0000 \\
\hline MEX & 0.0000 & 0.0000 & 0.0000 & 0.0000 & 0.0000 & 0.0000 & 0.0000 & 0.0000 & 0.0000 & 0.0000 & 0.0000 \\
\hline CSR & 0.0000 & 0.0000 & 0.0000 & 0.0000 & 0.0000 & 0.0000 & 0.0000 & 0.0000 & 0.0000 & 0.0000 & 0.0000 \\
\hline ELS & 0.0000 & 0.0000 & 0.0000 & 0.0000 & 0.0000 & 0.0000 & 0.0000 & 0.0000 & 0.0000 & 0.0000 & 0.0000 \\
\hline HIS & 0.0000 & 0.0000 & 0.0000 & 0.0000 & 0.0000 & 0.0000 & 0.0000 & 0.0000 & 0.0000 & 0.0000 & 0.0000 \\
\hline HON & 0.0000 & 0.0000 & 0.0000 & 0.0000 & 0.0000 & 0.0000 & 0.0000 & 0.0000 & 0.0000 & 0.0000 & 0.0000 \\
\hline MAR & 0.0000 & 0.0000 & 0.0000 & 0.0000 & 0.0000 & 0.0000 & 0.0000 & 0.0000 & 0.0000 & 0.0000 & 0.0000 \\
\hline MET & 0.0000 & 0.0000 & 0.0000 & 0.0000 & 0.0000 & 0.0000 & 0.0000 & 0.0000 & 0.0000 & 0.0000 & 0.0000 \\
\hline PRA & 0.0000 & 0.0000 & 0.0000 & 0.0000 & 0.0000 & 0.0000 & 0.0000 & 0.0000 & 0.0000 & 0.0000 & 0.0000 \\
\hline BRA & 0.0000 & 0.0000 & 0.0000 & 0.0000 & 0.0000 & 0.0000 & 0.0000 & 0.0000 & 0.0000 & 0.0000 & 0.0000 \\
\hline CAU & 0.0000 & 0.0000 & 0.0000 & 0.0000 & 0.0000 & 0.0000 & 0.0000 & 0.0000 & 0.0000 & 0.0000 & 0.0000 \\
\hline GRE & 0.0000 & 0.0000 & 0.0000 & 0.0000 & 0.0000 & 0.0000 & 0.0000 & 0.0000 & 0.0000 & 0.0000 & 0.0000 \\
\hline POR & 0.0000 & 0.0000 & 0.0000 & 0.0000 & 0.0000 & 0.0000 & 0.0000 & 0.0000 & 0.0000 & 0.0000 & 0.0000 \\
\hline SPN & 0.0000 & 0.0000 & 0.0000 & 0.0000 & 0.0000 & 0.0000 & 0.0000 & 0.0000 & 0.0000 & 0.0000 & 0.0000 \\
\hline SWE & 0.0000 & 0.0000 & 0.0000 & 0.0000 & 0.0000 & 0.0000 & 0.0000 & 0.0000 & 0.0000 & 0.0000 & 0.0000 \\
\hline
\end{tabular}

Refer to Table 1 for key to abbreviations

Pair-wise population comparisons yielding statistically insignificant differences both before (in bold) and after (in italics) applying the Bonferroni correction

\section{Supplementary Table 7: G-test (Cont'd)}




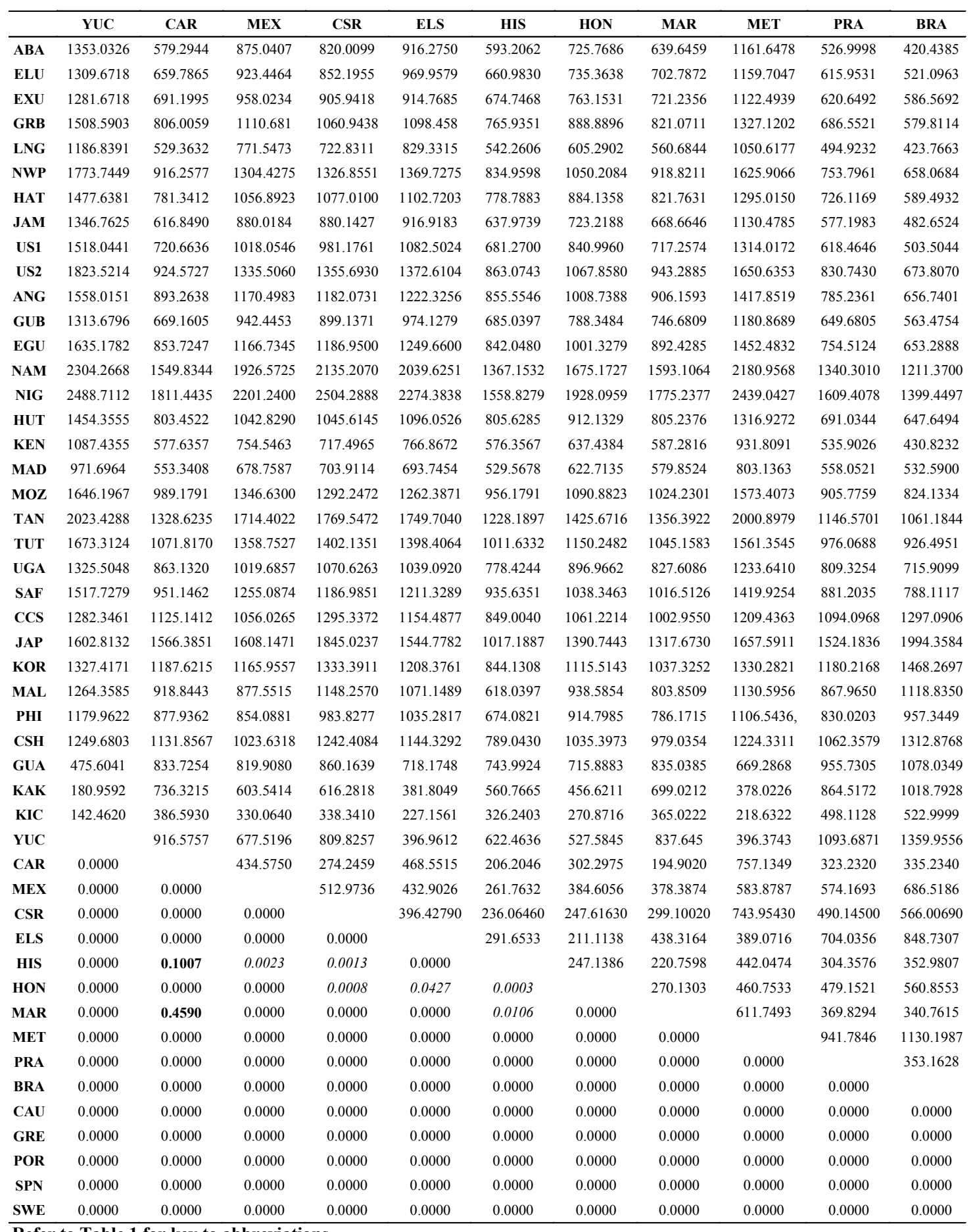

Refer to Table 1 for key to abbreviations

Pair-wise population comparisons yielding statistically insignificant differences both before (in bold) and after (in italics) applying the Bonferroni correction

Supplementary Table 7: G-test (Cont'd) 


\begin{tabular}{|c|c|c|c|c|c|}
\hline & CAU & GRE & POR & SPN & SWE \\
\hline $\mathbf{A B A}$ & 835.9993 & 829.8376 & 831.4743 & 915.6335 & 933.9509 \\
\hline ELU & 1001.3349 & 944.7720 & 951.8909 & 1067.2238 & 1091.9521 \\
\hline EXU & 998.6556 & 961.8715 & 996.8214 & 1090.2428 & 1105.3458 \\
\hline GRB & 1159.6194 & 1076.8927 & 1129.9086 & 1238.5070 & 1246.4890 \\
\hline LNG & 738.1310 & 708.7573 & 702.9459 & 770.1165 & 788.1989 \\
\hline NWP & 1319.9277 & 1303.3640 & 1352.7836 & 1462.1938 & 1490.2942 \\
\hline HAT & 1138.1791 & 1100.8298 & 1110.3181 & 1187.7039 & 1243.0212 \\
\hline JAM & 978.4950 & 896.0058 & 976.6739 & 1039.3077 & 1088.1926 \\
\hline US1 & 1044.4484 & 1008.4053 & 1072.1235 & 1147.5273 & 1184.6985 \\
\hline US2 & 1413.5024 & 1340.9468 & 1439.9379 & 1548.8081 & 1564.6559 \\
\hline ANG & 1275.2731 & 1230.3677 & 1293.5306 & 1355.2976 & 1345.2188 \\
\hline GUB & 1036.7103 & 983.4028 & 1017.8761 & 1098.9459 & 1143.3224 \\
\hline EGU & 1275.5774 & 1198.9954 & 1221.1036 & 1311.7319 & 1373.1744 \\
\hline NAM & 2128.6575 & 1976.3612 & 2115.2041 & 2229.7070 & 2229.2861 \\
\hline NIG & 2569.1582 & 2369.3403 & 2599.3826 & 2681.0117 & 2532.1086 \\
\hline HUT & 1098.8838 & 1061.8978 & 1087.2113 & 1185.1730 & 1186.5238 \\
\hline KEN & 835.1530 & 803.4288 & 814.1584 & 891.3480 & 922.3879 \\
\hline MAD & 863.2474 & 733.5222 & 859.1796 & 908.0583 & 960.3611 \\
\hline MOZ & 1439.9630 & 1357.5609 & 1440.5740 & 1557.8480 & 1609.4237 \\
\hline TAN & 1900.5359 & 1829.0458 & 1953.1154 & 2074.0374 & 2029.2335 \\
\hline TUT & 1480.4247 & 1444.9843 & 1482.8862 & 1564.5051 & 1584.5837 \\
\hline UGA & 1078.6869 & 1083.7622 & 1120.7635 & 1225.9478 & 1160.2367 \\
\hline SAF & 1347.4409 & 1285.5853 & 1322.6709 & 1425.7090 & 1507.3118 \\
\hline CCS & 1356.3972 & 1175.9008 & 1421.0850 & 1384.5605 & 1449.6512 \\
\hline JAP & 2086.0447 & 1828.4130 & 2348.3293 & 2244.5029 & 2302.3438 \\
\hline KOR & 1462.3920 & 1306.5034 & 1630.5028 & 1547.2969 & 1592.0238 \\
\hline MAL & 1192.6473 & 1006.2543 & 1250.0984 & 1216.8807 & 1347.4709 \\
\hline PHI & 1044.2216 & 898.5118 & 1089.7184 & 1085.7383 & 1137.3915 \\
\hline CSH & 1388.6058 & 1267.3691 & 1526.7745 & 1476.5314 & 1516.7231 \\
\hline GUA & 1216.2964 & 1178.3501 & 1290.5380 & 1259.0676 & 1267.4799 \\
\hline KAK & 1112.8190 & 1131.9576 & 1202.5743 & 1239.4248 & 1155.2915 \\
\hline KIC & 562.7928 & 576.3088 & 596.9670 & 625.5599 & 566.8100 \\
\hline YUC & 1517.5282 & 1538.1063 & 1663.1080 & 1710.7399 & 1549.4232 \\
\hline CAR & 503.3499 & 507.6903 & 499.8065 & 527.7271 & 669.8088 \\
\hline MEX & 783.9633 & 738.6413 & 846.1116 & 840.4451 & 873.8793 \\
\hline CSR & 685.68180 & 702.45790 & 750.1179 & 785.60460 & 840.07780 \\
\hline ELS & 1021.2999 & 1013.9773 & 1156.3560 & 1169.0570 & 1162.2632 \\
\hline HIS & 451.7975 & 485.5252 & 481.1879 & 510.1093 & 567.4726 \\
\hline HON & 727.9613 & 707.3621 & 767.3145 & 831.9730 & 814.4814 \\
\hline MAR & 423.3698 & 457.9126 & 450.6965 & 461.2663 & 526.8037 \\
\hline MET & 1256.2998 & 1235.3875 & 1403.1581 & 1436.8680 & 1314.5502 \\
\hline PRA & 509.8419 & 533.2666 & 507.1979 & 528.5404 & 641.0922 \\
\hline BRA & 528.9551 & 555.2408 & 505.0330 & 570.0999 & 556.5077 \\
\hline CAU & & 370.04950 & 286.5906 & 287.8519 & 288.3658 \\
\hline GRE & 0.0000 & & 312.2703 & 343.1823 & 483.5353 \\
\hline POR & 0.0000 & 0.0000 & & 220.5439 & 436.4818 \\
\hline SPN & 0.0000 & 0.0000 & 0.0092 & & 441.1469 \\
\hline SWE & 0.0000 & 0.0000 & 0.0000 & 0.0000 & \\
\hline
\end{tabular}

Refer to Table 1 for key to abbreviations

Pair-wise population comparisons yielding statistically insignificant differences both before (in bold) and after (in italics) applying the Bonferroni correction

\section{Supplementary Table 7: G-test (Cont'd)}




\section{THE PATRIARCHAL GENETIC SUBSTRATA OF THE BAHAMIAN ARCHIPELAGO}

\section{A. Introduction}

Historical records indicate that the indigenous people of the Bahamas were the Arawak (Lucayan) Indians (Bahamas Department of Archives, 1996), a group of Amerindians who traveled north from the Amazon River Basin and settled throughout the archipelago during the Ostionoid expansion (600-1100 AD) (Berman and Gnivecki, 1995; Keegan, 1995). Although this group resided in the island chain at the time of the discovery of the New World, most had perished by the early 1500s (Granberry, 1981; Johnson, 1996), leaving the Bahamas virtually uninhabited (Miller 1945, Lawlor 1998). The islands remained deserted until 1648 when a group of British independents from Bermuda migrated to the archipelago in search of religious freedom (Lawlor, 1998). The pilgrims, who were subsequently joined by other Bermudian colonists, of both African and European descent, established settlements on the island of Eleuthera and later on, in New Providence (Saunders, 1991).

In the following century, the Bahamas was raided over thirty times by French and Spanish pirates (Saunders, 1990), a period that would not end until the signing of the Treaty of Versailles in 1783, which granted the Bahamas to the British Empire (Williams, 1983). As a result, the Loyalists (a group of American colonists originating from Great Britain), fled continental North America for the Bahamas between 1783 and 1785 (the Bahamian Revolution). Prior to their arrival, African and European migrants were almost equally represented throughout the island chain (Saunders, 1985) yet, by 1788 the 
population had doubled, with Africans outnumbering Europeans 2:1 (Sharer, 1955). The escalation of the African component resulted from the influx of Creole slaves [individuals of African ancestry born in the New World (Saunders, 1985)] that accompanied the Loyalists and the importation of African-born individuals prior to the abolition of the British slave trade (Tinker, 1998). In addition, $\sim 6,000$ enslaved Africans, most of whom were aboard Portuguese slavers en route to Cuba, were captured by the British Royal Navy in the post slavery era (1807-1860) and resettled in the Bahamas (Dalleo, 1984; Tinker 1998), further contributing to the growth of the African populace.

During the post-emancipation era, the Bahamas, in contrast to other British Caribbean societies, experienced a surplus rather than a deficit in the labor force due to the collapse of the cotton industry in the early 1800 s and the importation of thousands of liberated Africans after 1807 (Saunders, 2003a). Consequently, the Bahamas, unlike the sugar-producing colonies (e.g., British Guiana, Jamaica and Trinidad), was not reliant upon indentured servitude (1838-1918) for the recruitment of hundreds of thousands of Asian laborers (Richardson, 1989). The archipelago did, however, receive migrants belonging to several different ethnic groups, although the extent of their genetic contributions is not known. Of the non-West Indian immigrants incorporated into Bahamian society, the Chinese were the first to make their mark, followed by the Greeks who traveled to the island chain seeking employment in the prospering Bahamian sponge trade. The 1920's bore witness to the introduction of Eastern European Jews, a second wave of Chinese migrants (by way of Cuba), as well as a small group of Lebanese nationals seeking religious asylum in the New World (Johnson, 1991; McCartney, 2004). Significant numbers of West Indians from Turks and Caicos, Barbados, Jamaica, Cuba 
and Guyana were also brought to the Bahamas from the 1920s onward to fulfill roles in law enforcement, education and construction. In addition to the aforementioned groups, Haitians have and continue to colonize the Bahama Islands as illegal immigrants (Tinker, 1998), comprising $7.1 \%$ of the total Bahamian population in the year 2000 (Department of Statistics of the Bahamas, 2000).

Studies characterizing the genetic structure of populations residing throughout the Caribbean basin have revealed differential African, European and Native American contributions to the maternal and/or paternal gene pools of each island sampled. In the recent report published by Benn-Torres and collaborators (2007), for example, high frequencies (72.0\% - 97.9\%) of the sub-Saharan African-specific mtDNA haplogroup L (sub-clades L0, L1, L2 and L3) were observed in Grenada, St. Kitts, St. Lucia, Dominica, St. Vincent and Trinidad, whereas the remaining influences in each collection's matrilineages originate from Eurasian (haplogroups $\mathrm{H}, \mathrm{K}, \mathrm{M}, \mathrm{N}$ and $\mathrm{U}$ ) and, in the case of the latter three populations, Native American (haplogroups A, C, and D) sources (Benn Torres et al., 2007). In the same study, Grenada, St. Kitts, St. Vincent, Trinidad, Jamaica and St. Thomas were found to share the majority (ranging from $58.3 \%$ to $88.3 \%$ ) of their paternal component with continental Africans, however, these estimates were generated based exclusively on Y-STR haplotype data. The Cuban populace, in contrast, displays sex-biased gene flow, receiving much larger genetic contributions from European males (78.8\%) than females (21.6\%) (Mendizabal et al., 2008), while collections from Aruba (Toro-Labrador, Wever and Martínez-Cruzado, 2003) and Puerto Rico (MartínezCruzado et al., 2005) are characterized by a predominantly Amerindian-specific ( $81.3 \%$ and $61.1 \%$, respectively) maternal component (i.e., haplogroups A, B, C and D). 
Despite the wealth of genetic data available for Caribbean and other New World populations, the islands of the Bahamas remain virtually unexplored genetically, with only a few phylogenetic and evolutionary studies on the island chain reported thus far. These investigations, which are based exclusively on classical (Halberstein et al., 1981) and autosomal STR (Duncan et al., 1996; Budowle et al, 1999; Herrera et al., 2004; Simms et al., 2008; 2010; 2011) polymorphisms, reveal substantial gene flow from continental Africa and varying degrees of genetic input from European, East Asian and Native American sources. However, given the high rate of recombination of autosomal STRs and the lack of resolution provided by classical markers, I explore here, for the first time, the paternal genetic histories of six geographically well-characterized Bahamian groups and their relationships with previously published reference collections using highresolution Y-chromosome binary markers. Considering the unique biology of the human Y-chromosome (i.e., selective neutrality, low mutation rate, stability and overall lack of recombination), it is a powerful tool for delineating genetic associations among closely related populations such as those examined in this report. In addition, I provide seventeen loci Y-STR haplotypes for three of the most prominent haplogroups (E1b1a7a-U174, E1b1a8-U175 and R1b1b1-M269) in order to assess the diversity within each of the six Bahamian islands and elucidate possible associations with specific source populations and/or migratory events. 


\section{B. Materials and Methods}

\section{Sample Collection and DNA Extraction}

Buccal swabs were collected from 428 unrelated male donors residing in the central [Grand Bahama (58) and Abaco (66)] and northwest portions [New Providence (142), Eleuthera (60), Exuma (59) and Long Island (43)] of the Bahamian archipelago. Genealogical information from each sampled individual was recorded for a minimum of two generations to establish paternal ancestry. All samples were procured with informed consent following the ethical guidelines stipulated by the Institutional Review Board of Florida International University. DNA was extracted using the Gentra Buccal Cell Kit (Puregene, Gentra Systems, Minneapolis, MN) according to the manufacturer's recommended protocols and quantitated with a NanoDrop 2000 spectrophotometer (NanoDrop products, Wilmington, DE) prior to PCR amplification.

\section{Y-chromosome haplogrouping}

A total of 136 binary markers were examined in hierarchical order by standard methods, including PCR-RFLP (Luis et al., 2004), allele-specific PCR (Gayden et al., 2008), size detection of the Y polymorphic Alu (YAP) insertional element (Hammer and Horai, 1995) or direct sequencing (Underhill et al., 2000). Y-haplogroup nomenclature is in accordance with Karafet et al. (2008) and recent updating by Myres et al. (2011). 
For comparative purposes, sample collections from West [Benin (Fon) and Cameroon (Bantu)], Central [Rwanda (Hutu)] and East [Kenya (Bantu)] Africa analyzed in a previously published report (Luis et al., 2004) were also included in the present study. The M191 derived individuals were tested for U174, P9.2, P115 and P116, while those reported as E3a*-M2 were typed for U175 and its downstream mutations (P278, U290, P59 and U181).

\section{Population Comparisons}

The Y-chromosome haplogroup variation among the six Bahamian populations and the 55 geographically targeted reference collections gathered from the literature (Table 1) was assessed through calculation of pairwise $F_{s t}$ distances using Arlequin version 3.11 (Excoffier et al., 2005). The $F_{s t}$ distances generated were represented in a multidimensional scaling (MDS) graph with the aid of PASW Statistics version 18 (SPSS, Inc., Chicago, IL, 2009). The same analysis software was also employed to quantify admixture for the Bahamian and other New World collections (i.e., Cuba, Nicaragua, South Argentina as well as Hispanic Americans, European Americans and African Americans from the United States) using the grouped African, European, East Asian and Native American populations as parental sources (Table 1). All analyses performed were based on the observed haplogroup frequencies at the resolution of the major haplogroups (A-T) of the Y-chromosome phylogram. 


\section{Y-STR genotyping}

Samples under the SNP backgrounds of E1b1a7a-U174, E1b1a8-U175 and R1b1b1-M269 were typed for 17 Y-STR loci (DYS19, DYS385a/b, DYS389I/II, DYS390, DYS391, DYS392, DYS393, DYS437, DYS438, DYS439, DYS448, DYS456, DYS458, DYS635, and Y-GATA H4) in a multiplex reaction using the AmpFlSTR ${ }^{\circledR}$ Yfiler Amplification Kit (Applied Biosystems, Foster City, CA). PCR products were electrophoresed on an ABI 3130xl Genetic Analyzer utilizing ABI GeneScan ${ }^{\mathrm{TM}} 500$ LIZ as the internal size standard. The GeneMapper ${ }^{\circledR}$ software v3.2 was employed to analyze the resulting Y-STR profiles through comparisons to the allelic ladder supplied by the manufacturer. Seventeen loci Y-STR haplotypes for individuals under the background of E1b1a7a, E1b1a8 and R1b1b1 are provided in Supplementary Tables 1-3, respectively.

\section{Phylogenetic and Statistical Analyses}

Haplotype diversity (HD), the number of haplotypes, the number of unique haplotypes and the mean number of pairwise differences were calculated using the Arlequin package version 3.11. The diversity indices generated were based on the 10 STR markers (DYS19, DYS389I, DYS389II, DYS390, DYS391, DYS392, DYS393, DYS437, DYS438 and DYS439) common to all collections included in the analyses. The same dataset was also utilized in the construction of multi-population networks with the aid of NETWORK version 4.5.1.6. Networks based on the Y-STR profiles of E1b1a7a, E1b1a8 and R1b1b1 individuals were calculated using the RM-MJ technique described in the program's user guide, available at http://www.fluxus-engineering.com. The Y-STR markers employed in the analyses were assigned weights as described previously 
(Berniell-Lee et al., 2009), $\varepsilon$ was set to zero and the "frequency $>1$ " criterion was activated. To further reduce network complexity, the postprocessing option, which employs a maximum parsimony (MP) calculation, was selected. MDS plots based on YSTR frequency distributions were also generated as described earlier.

For all Y-STR based analyses, the allele repeat length for DYS389b was computed by subtracting the number of repeats at DYS389II from the number at DYS389I and samples exhibiting duplicated loci, microvariant and/or null alleles were excluded. Also, to allow inclusion of pertinent European reference collections, the Athey’s Haplogroup Predictor 23-Haplogroup Beta program (Athey, 2006) was employed to infer haplogroup assignments. Only those samples yielding confidence estimates $\geq 90 \%$ were utilized for phylogenetic comparisons. Supplementary Tables 4-6 provide a list of SNP-STR references for haplogroups E1b1a7a, E1b1a8 and R1b1b1, respectively, along with the number of individuals utilized in the analyses.

\section{Results}

\section{Y-haplogroup distribution}

A total of 44 paternal lineages (Fig. 1) distributed throughout haplogroups A, B, DE*, E, G, I, J, Q, R and T were identified among the 428 Bahamian males analyzed in the present study. Of these, E-M96 (65.7\%), a clade detected at elevated levels in subSaharan Africa, is the most frequently observed haplogroup and is trailed by two European-specific lineages, R1b1b1-M269 (19\%) and I-M258 (6.5\%). Cumulatively, these three haplogroups constitute greater than $90 \%$ of the Y-chromosomes in the 
Bahamian gene pool. The geographic distribution of the major haplogroups (A-T) is illustrated in Figure 2A.

Haplogroup E1b1a-M2 is the most common of the E-M96 lineages in my dataset, accounting for greater than $50 \%$ of Bahamian Y-chromosomes. Although E1b1a-M2 lineages are shared across all six studied populations, they are detected at substantially lower levels $(23.3 \%)$ in the Long Island populace than in the collections from New Providence (60.6\%), Abaco (60.6\%), Exuma (62.7\%), Grand Bahama (63.8\%) and Eleuthera (68.3\%). It is interesting to note that haplogroups E1b1a7-M191 and E1b1a8U175, the two most abundant E1bla sub-lineages in Africa (Karafet et al., 2008), are almost equally represented in the latter five populations while in Long Island, the vast majority of M2 chromosomes (13.95\%) carry U175 derivatives (Fig. 2B). Appreciable frequencies of E1b1a* are also observed in all six collections, ranging from $7 \%$ in Long Island to $25 \%$ in Eleuthera.

Within the E1b1a7 sub-clade, the majority of Bahamian males are characterized by the U174 mutation, a trend that is also observed in the East and West African populations typed in the current report (Table 2). Interestingly, the sole Central African representative (Rwanda Hutus) was found to exhibit the highest proportion $(23.2 \%)$ of undifferentiated M191 Y-chromosomes, i.e., E1b1a7* lineages. The same group, along with the Bantu speakers from Cameroon, also displays the highest frequencies of the P116 derivative (13\% and 7.1\%, respectively). The E1b1a8 derived chromosomes, on the other hand, are predominated by the U290 mutation (haplogroup E1b1a8a1) in both the Bahamian and continental African collections, with the exception of Benin, which possesses a greater frequency $(15.7 \%)$ of E1b1a8a*-P278 lineages. Even though all U290 
derived samples were tested for U181 (a downstream mutation), this marker was observed only in Exuma, Grand Bahama and New Providence. Finally, the E1b1a8a2 sub-clade, which is present in only one of the Bahamian collections examined (i.e., New Providence, 2.1\%), is exclusive to the Bantu population from Kenya (3.5\%).

Haplogroups E1a, E2b and E1b1b (defined by the M33, M98 and M35 mutations, respectively), although not as abundant as E1bla lineages, are also present (Fig. 2B). Of the three sub-clades, E2b is by far the most common, with the highest levels (7\%) detected in the Exuma populace. Within this branch, M85* Y-chromosomes typically predominate over all other M98 patrilineages in the Bahamian gene pool, although two individuals (one in Exuma and one in New Providence) have also acquired the M200 mutation (Fig. 2B). This E2b derivative, to the authors' knowledge, has thus far been detected only in Mbuti Pygmies from the Democratic Republic of the Congo (Cruciani et al., 2002).

The B-M60 derived Y-chromosomes, on the other hand, which are reported at elevated levels in the Pygmy, Nilo-Saharan, Khoisan, and, to a lesser extent, Bantu populations of Africa (Wood et al., 2005; Gomes et al., 2010), are detected in the Bahamian gene pool at a frequency of $4 \%$. In each population studied, the vast majority of B-M60 derived individuals are represented by the B2a-M150 sub-clade and its downstream derivatives (M218 and M109), whereas the paralogous branch (B1-M236) is restricted to the collections from Abaco (3\%) and New Providence (2.1\%). It is interesting to note that four of the six Bahamian populations are also characterized by one sample bearing only the defining M60 mutation (i.e., B*-M60). 
Another haplogroup shared across all six studied populations is R1b1b1-M269, a lineage present at elevated levels in Western Europe (Meyers et al., 2011). In the present study, the majority of these Bahamian Y-chromosomes belong to haplogroups R1b1b1a1a-M405 and R1b1b1a1b2-M529, which occur at an average frequency of 5.6\% and $8.4 \%$, respectively. However, when the distribution of these two branches are compared, greater frequencies of M529 lineages are noted in the sample collections from Exuma (5.1\%), Eleuthera (6.7\%), Grand Bahama (6.9\%), New Providence (9.9\%) and Long Island (18.6\%), while higher proportions of M405/U106 derivatives are detected in Abaco (10.6\%). Interestingly, the Long Island populace, which harbors the highest frequencies of the above mentioned sub-clades, is also characterized by relatively high percentages of R1blbla1b*-S116 (9.3\%) and R1b1b1alb1-U152 (14\%) derived Ychromosomes. Outside of haplogroup R1b1b1, the remaining R-M306 derived samples were assigned to the R1a1a*-M198 (2.3\%) and R1b2*V88 (3\%) sub-clades, which are present at elevated levels in South Asia (Underhill et al., 2010) and Cameroon (Cruciani et al., 2010), respectively.

The other important European haplogroup, I-M258, was detected in the combined Bahamian dataset at a frequency of $6.5 \%$. Of this haplogroup's sub-clades, the I1 branch (typical of Northern Europeans (Rootsi et al., 2004)) was found to predominate I lineages in Eleuthera (6.7\%), Grand Bahama (5.2\%) and Long Island (4.7\%) whereas the frequency of haplogroup I2 derivatives (characteristic of Southeast European/Balkan populations (Battaglia et al., 2009)) was higher in the collections from Abaco (6.1\%) and Exuma (5.1\%). It is noteworthy that all of the Y-chromosomes within the latter branch (except for one individual in Exuma and one in Abaco) have acquired the M223, and in 
some cases, the M284 mutation, whereas those belonging to the former sub-clade bear only the M253 mutation and are therefore classified as I1*.

\section{Genetic Structure}

In the MDS plot (Fig. 3), the Bahamian (i.e., Abaco, Eleuthera, Exuma, Grand Bahama and New Providence) and US African American collections segregate to the right half of the projection, forming a loosely associated aggregate with Kenya (Bantu) and several of the West [Cameroon (Bantu), Gabon and Angola] and Central [Democratic Republic of the Congo (Hema), Central African Republic (Pygmies) and Botswana] African populations (denoted by the dashed circle in Fig. 3). Long Island, in contrast, displays a considerable degree of genetic separation from the other five Bahamian groups, partitioning on the opposite side of the graph with the European, Northeast African (although the Nilotes from Uganda and the Khoisan from South Africa are also included) and New World populations. The positioning of Abaco, New Providence, Grand Bahama, Eleuthera and Exuma amidst the continental Africans is anticipated given the high frequencies of haplogroup E lineages $(65.2 \%, 68.1 \%, 70.7 \%, 73.3 \%$ and $76.3 \%$, respectively) in these collections whereas the separation of Long Island is likely driven by elevated levels of M269 derived Y-chromosomes (55.8\%).

Admixture proportions for Abaco, Eleuthera, Exuma, Grand Bahama, Long Island and New Providence are provided in Table 3, along with those calculated for several other New World populations. The results indicate that five of the islands sampled have received the majority of their paternal lineages $(89.7 \%$ - 100\%) from African sources, while the remaining influences in each collection's gene pool emanate exclusively from 
Europeans. The Long Island populace is the exception, sharing $75.2 \%$ of its paternal component with the latter group of populations. Commensurate levels of European influences (ranging from $67.3 \%$ in Hispanic Americans to $97.4 \%$ in European Americans) were also observed in the other New World collections examined, but, in contrast to Bahamians (excluding Long Island) and US African Americans, these populations also receive genetic input from East Asian (1.40\% - 2.90\%) and Native American $(0.10 \%-16.5 \%)$ sources.

\section{Y-STR Distributions and Diversity}

Haplotype diversity (HD) for the Bahamian populations is high (ranging from 0.9341-1.0000, 0.9455-1.0000 and 0.9565-1.0000 for haplogroups E1b1a7a, E1b1a8 and R1b1b1, respectively) and haplotype sharing within the islands is limited, with the largest number of shared haplotypes equaling 10 (Long Island, haplogroup R1b1b1). In most instances, the Bahamian collections exhibit HD levels comparable to or even higher than those of the reference populations. Diversity indices for all populations examined within haplogroups E1b1a7a, E1b1a8 and R1b1b1 are provided in Supplementary Tables 7-9, respectively.

Phylogenetic networks representing Y-STR haplotype variation within haplogroups E1b1a7a and E1b1a8 are presented in Figures 4A and 4B, respectively. Haplotype distributions in the six Bahamian populations are widespread throughout both projections, sharing clusters with all three African groups employed in the analysis. In the E1b1a7a network, 24 different Y-STR haplotypes are in common among the Bahamian and continental African collections, with 19 of these are shared exclusively with West 
and/or Central Africans. Figure 4B, on the other hand, illustrates a greater number (32) of shared haplotypes, although the majority of these are also present in both the West and Central African populations. Several Bahamian-specific clades are also noted in both networks, further attesting to the high level of diversity observed in this group of populations.

Network analysis of the R1b1b1 samples (Fig. 5) demonstrates extensive haplotype sharing between the Bahamian and European populations, particularly with those from the United Kingdom, Ireland and the Iberian Peninsula. Although the Bahamian collections display stronger genetic affinities with West Europeans, they also possess Y-STR profiles in common with individuals from the Balkan Peninsula. In this projection, New Providence is the only Bahamian group to exhibit a cluster exclusively with Poland and the Balkan groups. Also, in comparison to the African-specific networks (Fig. 4A,B), a greater number of independent Bahamian clades are observed.

In the MDS projections based on the Y-STR allelic frequencies of individuals possessing haplogroup E1b1a7a (Fig. 6A) and E1b1a8 (Fig. 6B) derivatives, substantial variation among the six studied populations is apparent. When only the E1b1a7a derived samples are considered (Fig. 6A), Eleuthera and Exuma form a loosely associated cluster in the upper portion of the graph with the populations from Gabon, Cameroon (Bantu), Benin, Nigeria, Ivory Coast, Uganda and Central African Republic (Pygmy). In the lower half of the plot, Abaco lies adjacent to several of the Central [Democratic Republic of the Congo (Bantu) and Zambia (Bantu)] and East [Tanzania (Bantu) and Kenya (NiloSaharan)] African groups, while New Providence and Grand Bahama map in closest proximity to Burkina Faso and Tanzania (Khoisan). However, in the case of haplogroup 
E1b1a8 (Fig. 6B), the MDS reveals genetic associations distinct from those for E1b1a7a. The collection from Eleuthera lies intermediate to Angola and the population pair consisting of Botswana and Tanzania (Khoisan) in the upper left quadrant, while Exuma maps in closest proximity to Kenya (Bantu) and Burkina Faso in the lower left section but also exhibits genetic affinities to the Bantu speakers from the Democratic Republic of the Congo. To the right of the projection, Long Island and Grand Bahama segregate together with Cameron (Bantu), occupying a transitional position between Uganda and the collection from Abaco, which plots as an outlier at the perimeter of the graph. Interestingly, the New Providence populace strays away from all other Bahamian groups, exhibiting genetic affinities with the Ivory Coast collection in the bottom-right quadrant.

\section{Discussion}

Genetic signatures of the Transatlantic slave trade

The considerable proportion (ranging from $71.2 \%$ to $79.7 \%$ ) of haplogroup AM91, B-M60, E1a-M33, E1b1a-M2 and E2b-M98 lineages in five of the Bahamian populations analyzed (i.e., Abaco, Eleuthera, Exuma, Grand Bahama and New

Providence) signals a direct genetic connection with continental Africa, consistent with earlier reports based on classical (Halberstein et al., 1981) and autosomal STR (Herrera et al., 2004; Simms et al., 2008; 2010; 2011) markers. Genetic affinities between the two regions, which are also observed in the MDS (Fig. 3) and admixture analyses (Table 3), further corroborate historical accounts of gene flow from African and Creole slaves prior 
to the abolition of slavery in 1807 (Saunders, 1985; Johnson, 1996; Pepin, 2005) and from liberated Africans during the post-slavery era (Wood, 1974; Tinker 1998).

According to Pepin (2005), the largest proportion of slaves transported to the Bahamas during the period of the Transatlantic slave trade embarked from West-Central Africa (26.8\%), although substantial numbers also departed from ports along the Gold Coast $(20.2 \%)$, the Bight of Biafra (17.1\%) and the Windward Coast (14.6\%). Utilizing high-resolution Y-chromosome binary markers, I was able to uncover genetic associations between the Bahamian collections and those originating in West and Central Africa not only in MDS graph (Fig. 3) but also with respect to NRY haplogroup distributions. Particularly suggestive of this close genetic relationship is the observed prominence of sub-Saharan specific E1b1a7-M191 and E1b1a8-U175 lineages in the Bahamas, with sub-haplogroups E1b1a7a-U174 and E1b1a8a1-U290 accounting for the majority of Y-chromosomes (with the exemption of Rwanda and Benin, respectively) in the Bahamian, West and Central African populations (Table 2). P116, a downstream mutation of E1b1a7a-U174, provides additional support for this notion, with low frequencies of this marker being observed in the collections from Abaco, Eleuthera, Exuma and New Providence as well as those from Cameroon (Bantu) and Rwanda (Hutus) (Table 2). Moderate levels of undifferentiated M2* chromosomes throughout the archipelago also allude to genetic signatures from West Africa, with the Mande speakers from Senegal and Burkina Faso (de Filippo et al., 2011) displaying the highest frequencies and groups from Nigeria, Ghana and Cameroon exhibiting appreciable proportions (Veeramah et al., 2010). 
The distribution of Y-STR haplotypes within haplogroups E1b1a7a-U174 and E1b1a8-U175, as illustrated in the Network (Fig. 4A and 4B, respectively) and MDS projections (Fig. 6A and 6B, respectively) as well as in the substantially high HD values (Supplementary Tables 7 and 8, respectively), is strikingly diverse, suggesting that the Bahamian archipelago has been influenced by a wide array of African source populations. This finding is not surprising given that the Africans settling in the Bahamas were members of various tribes, including, but not limited to, the Igbo/Ebo, Mandingo, Fulani, Hausa (Albury, 1975), Yoruba, Congo (Albury, 1975; Duncan et al. 1996), Akan (Wilkie, 1993), Kru, Ndongo and Mbundu (Saunders, 1996) peoples, originating throughout West and Central Africa. It is important to note that many cultural influences derived from these groups (e.g., music (Goombay, Junkanoo), dance (Ring play), storytelling, obeah (black and white magic), 'bush' medicine and even the communal practice of money management, the 'Asue') still remain a part of Bahamian tradition (Saunders, 1995).

Genetic signals from East Africa, albeit not as prominent, are also noted in the current investigation. In both the U174 (Fig. 4A) and U175 (Fig. 4B) networks, a considerable number of Y-STR haplotypes are shared among the Bahamian, Central, West and East African populations, indicating a close genetic relationship among these four groups. Affinities with East Africans are also evident in the Y-STR based MDS plots (Fig. 6A, B) despite the fact that no Bahamian/East African specific clades are observed in either network projection. Genetic signatures linking the Bahamian and East African populations are likely relics of the Bantu expansion throughout sub-Saharan Africa (Plaza et al, 2004; Beleza et al, 2005), although several other explanations are plausible. These 
include: (1) the apprehension and shipment of captives from Southeast Africa to West African ports prior to disembarking for the New World (Thomas, 1998; Pereira et al., 2001) and (2) the resettlement of Africans aboard slave ships transporting mixed cargoes [i.e., slaves of Central, East and West African origins (Dalleo, 1984)] in the Bahamas (Tinker, 1998). According to the Collector of Customs' report in 1828, representatives of the Gamba and Hanga tribes of Sudan and Kenya, respectively, were present throughout the island chain (Dalleo, 1982).

\section{Gene flow from Western Europe}

Western European colonialism, although short-lived, has left marked genetic imprints throughout the Bahamian archipelago, with Long Island receiving the strongest European genetic signals and Exuma, the weakest; a distribution pattern consistent with my earlier reports utilizing autosomal STR markers (Simms et al., 2008; 2010; 2011). The relatively larger proportion of M269 derived Y-chromosomes in the Long Island population (55.8\%), as compared to the other five Bahamian islands surveyed (ranging from $8.5 \%-18.3 \%$ ), is likely attributed to higher rates of genetic admixture between European males and African females (Saunders, 2003b). According to the 1851 census, Long Island possessed one of the lowest European components (13.1\%) yet, by 1953, almost $50 \%$ of this population was of 'mixed' ancestry (Craton, 1998). While this may simply be the result of lower numbers of M269 chromosomes in the other five islands sampled, it may also reflect genetic drift due to the small effective population size of the Long Island European colonial fraction during the eighteenth century. This notion is 
further supported by Long Islands' low observed HD $(0.9565 \pm 0.0204)$ within haplogroup R1b1b1-M269.

Appreciable frequencies of haplogroups R1b1b1a1b2*-M529 [common in individuals of British or Irish ancestry (Myres et al., 2011)] and R1b1b1a1a*-M405 [typical of populations originating in Denmark, Germany (Cruciani et al., 2011), England, France and the Netherlands (Myres et al., 2011)] in the gene pools of the six Bahamian populations allude to genetic connections with Great Britain. Influences from this region, which are anticipated given the influx of British Independents from Bermuda in the mid 1600s and the thousands of British Loyalists from North America between 1783 and 1785, are also observed in the network of R1b1b1 individuals (Fig. 5). In this projection, 18 haplotypes are shared between the populations from the Bahamas and the United Kingdom (UK), with New Providence and Long Island forming a clade exclusively with this group. Considerable sharing of haplotypes (23) with the collections from the Iberian Peninsula is also noted, suggesting potential influences from Spain and/or Portugal. The presence of haplogroup R1b1b1a1b* in Abaco, Grand Bahama, Long Island and New Providence provides further support for this notion, as undifferentiated S116 Y-chromosome are almost exclusive to these two parts of Europe (Myres et al., 2011). Genetic affinities with populations originating in the Iberian Peninsula are likely the result of migratory events from Southern Europe, Latin America and/or the US (Department of Statistics of the Bahamas, 2000). 
Interestingly, the distribution of European genetic influences in the Bahamian populations analyzed mirrors Loyalists settlement patterns throughout the archipelago. Saunders (1983) explains that two classes of Loyalists migrated into the island chain: (1) the 'business men' (officers, merchants and professionals) that settled in New Providence and the Northern Bahamas [excluding Grand Bahama since it was not permanently settled until 1806 (Dold, Folster and Vaitlingam, 2003)] and (2) the 'farmers' that ventured to the south. Those taking up residence in the north, particularly in Abaco and north Eleuthera, were more inclined to intermarry with the Old residents (the Conchs) than to genetically mix with the slaves. As a result of these endogamous practices, a larger proportion of European Y-chromosomes appear to have been preserved in both collections' gene pool.

On the contrary, Loyalists settling in the Southern Bahamas (e.g., Long Island and Exuma) implemented the plantation system, using slave labor to grow Sea Island cotton (Saunders, 1983). However, with the collapse of the cotton industry, most of these Loyalists abandoned their plantations granting the land to their slaves (Craton and Saunders, 1992). This is the most likely explanation for the strong African and relatively weak European genetic signals displayed by the Exuma populace regardless of which marker system [autosomal (Simms et al., 2011) or Y-chromosome (Table 3)] is employed. Long Island, on the other hand, possessed a large number of poor Loyalists who could not afford to leave when cotton failed. Instead this group remained in the island and, over time, genetically mixed with the slaves to form a community of "nearwhites" (Craton, 1998). Therefore, it is not surprising that the admixture profile (Table 3) for Long Island identifies Europeans as the primary genetic contributor, although it is 
interesting to note that influences from this region are substantially higher in this population's paternal vs. autosomal gene pool, possibly indicating asymmetric mating between European males and African females (Saunders, 2003b).

Comparisons with the other New World populations

In the present study, the NRY haplogroup distributions of Abaco, Eleuthera, Exuma, Grand Bahama and New Providence were not found to differ considerably from other African derived groups in the New World. Elbla lineages, for example, are observed in the above mentioned islands (ranging from 60.6\% to 68.3\%) at frequencies comparable to those observed in African-Brazilian (Hünemeier et al., 2007; Gonçalves et al., 2008), Afro-Ecuadoran (González-Andrade et al., 2007), Barbadian (Harris et al., 2006) and US African American (Vallone and Butler, 2004; Sims, Garvey and Ballantyne, 2007) populations. Likewise, commensurate levels of E1b1a7-M191 (formerly classified as E3a7) derived chromosomes were detected in the Bahamian (at an average frequency of 20.3\%), US African American (Sims, Garvey and Ballantyne, 2007) and African-Brazilian (Gonçalves et al., 2008) groups, however, Bahamians and US African Americans (Sims, Garvey and Ballantyne, 2007) were the only ones typed for the E1b1a8-U175 sub-clade. These latter three collections also exhibit similar levels of E1a-M33 and E2-M75, while M35 derived chromosomes, which are characteristic of Southeastern European as well as North and East African populations, are considerably more frequent in Afro-Brazilians than in the aforementioned groups. 
The R1b1b1 lineages are also observed at commensurate levels in Bahamians (at an average frequency of 18.5\%), US African Americans (Hammer et al., 2006; Sims, Garvey and Ballantyne, 2007), Afro-Ecuadorans (González-Andrade et al., 2007) and Barbadians (Harris et al., 2006), although when downstream markers are examined the collections from Abaco, Eleuthera, Exuma, Grand Bahama exhibit higher levels of M405 derived chromosomes than the US African American population. The comparability of distributions of the two most common haplogroups, E and R, in the Bahamian and other New World African populations, especially US African Americans (Table 3), may represent genetic signatures from similar ancestral sources and/or episodes of gene flow between the different countries within the region. The Long Island populace, on the other hand, which possesses a much lower frequency (23.3\%) of M2 derived Y-chromosomes and a substantially higher proportion of R1b1b1 (58.1\%) lineages, exhibits a genetic profile typical of several Latin American groups in the New World [e.g., Brazilians (Porto Alegre) (Hünemeier et al., 2007) and Nicaraguans (Nuñez et al., 2010)].

\section{Genetic signals from other ethnic groups}

Over the last 150 years, the Bahamas has been witness to a rich and varied array of settlers, including Chinese immigrant workers, Greek spongers, Jewish businessmen and individuals of Lebanese descent fleeing religious persecution. The extent to which each group has contributed genetically to the Bahamian paternal gene pool, however, is unknown. My findings suggest that the Greeks, which exhibit relatively high frequencies of haplogroups E1b1a*-M78, J2a*-M410 and R1b1b1*-L23 (Myres et al., 2011; Semino et al., 2004), are a likely source of these lineages in the Bahamas, although the presence 
of M78 derived chromosomes may also signal gene flow from Lebanon (Zalloua et al., 2008). J1e-P58 lineages, on the other hand, which are characteristic of Jewish (Hammer et al., 2009) and Arab speaking groups (Chiaroni et al., 2009), may represent genetic signatures of Eastern European Jews and/or Lebanese migrants entering the Bahamas in the early twentieth century. Interestingly, the Native American and East Asian genetic signals detected in my earlier study using autosomal STR makers (Simms et al., 2011) are completely absent from the paternal gene pool of each island analyzed (Table 3). The lack of genetic input from these groups may reflect smaller sample sizes and/or sexbiased gene flow.

\section{E. Conclusion}

My findings support a stronger genetic relationship between Bahamians (excluding Long Island) and continental Africans than with any other group examined. This close genetic affinity, particularly with the West and Central African populations, is most likely attributed to the immigration of African and Creole slaves into the archipelago by the Loyalists and the thousands of liberated Africans resettled in the Bahamas by the British during the post slavery era. The Long Island collection, in contrast to the other five Bahamian islands analyzed, derives the majority of its paternal component from Europeans, possibly the result of asymmetric mating between European males and African females on this island. Additional investigations of the six studied populations using mtDNA polymorphisms should provide further understanding of the genetic history of the Bahamas. 


\section{REFERENCES}

Albury, P. 1975. The story of the Bahamas. London: Macmillan Education Limited.

Alonso S, Flores C, Cabrera V, Alonso A, Martín P, Albarrán C, Izagirre N, de la Rúa C, García O. 2005. The place of the Basques in the European Y-chromosome diversity landscape. Eur J Hum Genet 13:1293-1302.

Alves C, Gomes V, Prata MJ, Amorim A, Gusmão L. 2007. Population data for Ychromosome haplotypes defined by 17 STRs (AmpFlSTR YFiler) in Portugal. Forensic Sci Int 171:250-255.

Applied Biosystems. 2006. AmpFlSTR Yfiler PCR amplification kit user's manual. Foster City, CA: Applied Biosystems.

Athey W. 2006. Haplogroup prediction from Y-STR values using a Bayesian-allelefrequency approach. J Genet Geneal 2:34-39.

Bahamas Department of Archives. 1996. The Peoples of the Bahamas. Nassau, Bahamas: Government Printing Department.

Ballard DJ, Phillips C, Thacker CR, Robson C, Revoir AP, Syndercombe Court D. 2005. Y chromosome STR haplotypes in three UK populations. Forensic Sci Int 152:289-305.

Ballard DJ, Phillips C, Thacker CR, Court DS. 2006. Y chromosome STR haplotype data for an Irish population. Forensic Sci Int 161:64-68.

Battaglia V, Fornarino S, Al-Zahery N, Olivieri A, Pala M, Myres NM, King RJ, Rootsi S, Marjanovic D, Primorac D, Hadziselimovic R, Vidovic S, Drobnic K, Durmishi N, Torroni A, Santachiara-Benerecetti AS, Underhill PA, Semino O. 2009. Y-chromosomal evidence of the cultural diffusion of agriculture in southeast Europe. Eur J Hum Genet 17:820-830.

Beleza S, Gusmão L, Amorim A, Carracedo A, Salas A. 2005. The genetic legacy of western Bantu migrations. Hum Genet 117:366-375.

Beleza S, Gusmão L, Lopes A, Alves C, Gomes I, Giouzeli M, Calafell F, Carracedo A, Amorim A. 2006. Micro-phylogeographic and demographic history of Portuguese male lineages. Ann Hum Genet. 70:181-194.

Benn Torres J, Kittles RA, Stone AC. 2007. Mitochondrial and Y chromosome diversity in the English-speaking Caribbean. Ann Hum Genet 71:1-9.

Berman M J, Gnivecki P L. 1995. The colonization of the Bahamas archipelago: a reappraisal. World Archaeology 26:421-441. 
Berniell-Lee G, Sandoval K, Mendizabal I, Bosch E, Comas D. 2007. SNPlexing the human Y-chromosome: a single-assay system for major haplogroup screening. Electrophoresis 28:3201-3206.

Berniell-Lee G, Calafell F, Bosch E, Heyer E, Sica L, Mouguiama-Daouda P, van der Veen L, Hombert J, Quintana-Murci L, Comas D. 2009. Genetic and demographic implications of the Bantu expansion: insights from human paternal lineages. Mol Biol Evol 26:1581-1589.

Bosch E, Calafell F, González-Neira A, Flaiz C, Mateu E, Scheil HG, Huckenbeck W, Efremovska L, Mikerezi I, Xirotiris N, Grasa C, Schmidt H, Comas D. 2006. Paternal and maternal lineages in the Balkans show a homogeneous landscape over linguistic barriers, except for the isolated Aromuns. Ann Hum Genet 70:459-487.

Brión M, Sobrino B, Blanco-Verea A, Lareu MV, Carracedo A. 2004. Hierarchical analysis of 30 Y-chromosome SNPs in European populations. Int J Legal Med 119:10-15.

Brión M, Sanchez JJ, Balogh K, Thacker C, Blanco-Verea A, Børsting C, StradmannBellinghausen B, Bogus M, Syndercombe-Court D, Schneider PM, Carracedo A, Morling N. 2006. Analysis of 29 Y-chromosome SNPs in a single multiplex useful to predict the geographic origin of male lineages. Int Congr Ser 1288:13-15.

Brucato N, Cassar O, Tonasso L, Tortevoye P, Migot-Nabias F, Plancoulaine S, Guitard E, Larrouy G, Gessain A, Dugoujon JM. 2010. The imprint of the Slave Trade in an African American population: mitochondrial DNA, Y chromosome and HTLV-1 analysis in the Noir Marron of French Guiana. BMC Evol Biol 10:314.

Budowle B, Moretti T, Baumstark AL, Defenbaugh DA, Keys KM. 1999. Population data on the thirteen CODIS core short tandem repeat loci in African Americans, U.S. Caucasians, Hispanics, Bahamians, Jamaicans, and Trinidadians. J Forensic Sci 44:12771286.

Campbell KD. 2007. Geographic Patterns of Haplogroup R1b in the British Isles. JOGG 3:1-13.

Chiaroni J, King RJ, Myres NM, Henn BM, Ducourneau A, Mitchell MJ, Boetsch G, Sheikha I, Lin AA, Nik-Ahd M, Ahmad J, Lattanzi F, Herrera RJ, Ibrahim ME, Brody A, Semino O, Kivisild T, Underhill PA. 2010. The emergence of Y-chromosome haplogroup J1e among Arabic-speaking populations. Eur J Hum Genet 18:348-353.

Coelho M, Sequeira F, Luiselli D, Beleza S, Rocha J. 2009. On the edge of Bantu expansions: mtDNA, Y chromosome and lactase persistence genetic variation in southwestern Angola. BMC Evol Biol 9:80. 
Corach D, Lao O, Bobillo C, van Der Gaag K, Zuniga S, Vermeulen M, Van Duijn K, Goedbloed M, Vallone PM, Parson W, de Knijff P, Kayser M. 2010. Inferring continental ancestry of Argentineans from autosomal, Y-chromosomal and mitochondrial DNA. Ann Hum Genet 74:65-76.

Craton M. 1998. Bay street, black power and the conchy joes: race and class in the colony and Commonwealth of the Bahamas. In: Howard $\mathrm{H}$ and Watson K, editors. The white minority in the Caribbean. Kingston, Jamaica: Ian Randle Publishers. p 71-94.

Cruciani F, Santolamazza P, Shen P, Macaulay V, Moral P, Olckers A, Modiano D, Holmes S, Destro-Bisol G, Coia V, Wallace DC, Oefner PJ, Torroni A, Cavalli-Sforza LL, Scozzari R, Underhill PA. 2002. A back migration from Asia to sub-Saharan Africa is supported by high-resolution analysis of human Y-chromosome haplotypes. Am J of Hum Genet 70:1197-1214.

Cruciani F, La Fratta R, Santolamazza P, Sellitto D, Pascone R, Moral P, Watson E, Guida V, Colomb EB, Zaharova B, Lavinha J, Vona G, Aman R, Calì F, Akar N, Richards M, Torroni A, Novelletto A, Scozzari R. 2004. Phylogeographic analysis of haplogroup E3b (E-M215) Y chromosomes reveals multiple migratory events within and out of Africa. Am J Hum Genet 74:1014-1022.

Cruciani F, Trombetta B, Sellitto D, Massaia A, Destro-Bisol G, Watson E, Colomb EB, Dugoujon J, Moral P, Scozzari R. 2010. Human Y chromosome haplogroup R-V88: a paternal genetic record of early mid Holocene trans-Saharan connections and the spread of Chadic languages. Eur J Hum Genet 18:800-807.

Cruciani F, Trombetta B, Antonelli C, Pascone R, Valesini G, Scalzi V, Vona G, Melegh B, Zagradisnik B, Assum G, Efremov GD, Sellitto D, Scozzari R. 2011. Strong intra- and inter-continental differentiation revealed by Y chromosome SNPs M269, U106 and U152. Forensic Sci Int: Genet 5:e49-52.

Dalleo PT. 1982. African-Bahamian origins. J Bahamas Hist. Soc 4:17-19.

Dalleo PT. 1984. Africans in the Caribbean: a preliminary assessment of recaptives in the Bahamas 1811-1860. J Bahamas Hist Soc 6:15-24.

de Filippo C, Barbieri C, Whitten M, Mpoloka SW, Gunnarsdóttir ED, Bostoen K, Nyambe T, Beyer K, Schreiber H, de Knijff P, Luiselli D, Stoneking M, Pakendorf B. 2011. Y-chromosomal variation in Sub-Saharan Africa: insights into the history of NigerCongo groups. Mol Biol Evol 28:1255-1269.

Department of Statistics of the Bahamas, The 2000 census of population and housing tables (All Bahamas). http://statistics.bahamas.gov.bs/archives.php?cat=70. 
Dold G, Folster N, Vaitlingam A. 2003. Rough Guide to the Bahamas 1. London: Rough Guides.

Duncan G, Thomas E, Gallo JC, Baird LS, Garrison J, Herrera RJ. 1996. Human phylogenetic relationships according to the D1S80 locus. Genetica 98:277-287.

Excoffier L, Laval G, Schneider S. 2005. Arlequin ver 3.0: an integrative software package for population genetics data analysis. Evol Bioinform Online 1:47-50.

Flores C, Maca-Meyer N, González AM, Oefner PJ, Shen P, Pérez JA, Rojas A, Larruga JM, Underhill PA. 2004. Reduced genetic structure of the Iberian peninsula revealed by Y-chromosome analysis: implications for population demography. Eur J Hum Genet 12:855-863.

Granberry J. 1981. Spanish slave trade in the Bahamas, 1509-1530: an aspect of the Caribbean pearl industry (Last Part). J Bahamas Hist Soc 3:17-19.

Gayden T, Regueiro M, Martinez L, Cadenas AM, Herrera RJ. 2008. Human Ychromosome haplotyping by allele-specific polymerase chain reaction. Electrophoresis 29:2419-2423.

Gomes V, Sánchez-Diz P, Amorim A, Carracedo Á, Gusmão L. 2010. Digging deeper into East African human Y chromosome lineages. Hum Genet 127:603-613.

Gonçalves R, Spínola H, Brehm A. 2007. Y-Chromosome lineages in São Tomé e Príncipe islands: evidence of European influence. Am J Hum Biol 19:422-428.

Gonçalves VF, Carvalho CMB, Bortolini MC, Bydlowski SP, Pena SDJ. 2008. The phylogeography of African Brazilians. Hum Hered 65:23-32.

González-Andrade F, Sánchez D, González-Solórzano J, Gascón S, Martínez-Jarreta B. 2007. Sex-specific genetic admixture of Mestizos, Amerindian Kichwas, and AfroEcuadorans from Ecuador. Hum Biol 79:51-77.

Halberstein RA, Davies JE, Mack K. 1981. Hemoglobin variations on a small Bahamian island. Am J Phy Anthropol 55:217-221.

Hammer MF, Horai S. 1995. Y chromosomal DNA variation and the peopling of Japan. Am J Hum Genet 56:951-962.

Hammer MF, Chamberlain VF, Kearney VF, Stover D, Zhang G, Karafet T, Walsh B, Redd AJ. 2006. Population structure of Y chromosome SNP haplogroups in the United States and forensic implications for constructing Y chromosome STR databases. Forensic Sci Int 164:45-55. 
Hammer MF, Behar DM, Karafet TM, Mendez FL, Hallmark B, Erez T, Zhivotovsky LA, Rosset S, Skorecki K. 2009. Extended Y chromosome haplotypes resolve multiple and unique lineages of the Jewish priesthood. Hum Genet 126:707-717.

Harris KA, Thacker CR, Ballard D, Harrison Musgrave-Brown CE, Syndercombe Court D, SNP for ID consortium. 2006. An investigation into the genetic structure of a Barbadian population. Int Congr Ser 1288:412-414.

Hassan HY, Underhill PA, Cavalli-Sforza LL, Ibrahim ME. 2008. Y-chromosome variation among Sudanese: restricted gene flow, concordance with language, geography, and history. Am J Phys Anthropol 137:316-323.

Herrera RJ, Adrien LR, Ruiz LM, Sanabria NY, Duncan G. 2004. D1S80 single-locus discrimination among African populations. Hum Biol 76:87-108.

Hünemeier T, Carvalho C, Marrero AR, Salzano FM, Pena SDJ, Bortolini MC. NigerCongo speaking populations and the formation of the Brazilian gene pool: mtDNA and Y-chromosome data. Am J Phy Anthropol 133:854-867.

Johnson H. 1991. The Bahamas in slavery and freedom. Kingston, Jamaica: Ian Randle Publishing.

Johnson H. 1996. The Bahamas from slavery to servitude, 1783-1933.Gainesville, Florida: University Press of Florida.

Karafet TM, Mendez FL, Meilerman MB, Underhill PA, Zegura SL, Hammer MF. 2008. New binary polymorphisms reshape and increase resolution of the human Y chromosomal haplogroups tree. Genome Res 18:830-838.

Karafet TM, Hallmark B, Cox MP, Sudoyo H, Downey S, Lansing JS, Hammer MF. 2010. Major east-west division underlies Y chromosome stratification across Indonesia. Mol Biol Evol 27:1833-1844.

Keegan, W. 1995. Modeling dispersal in the prehistoric West Indies. World Archaeology 26:400-420.

Kovatsi L, Saunier JL, Irwin JA. 2009. Population genetics of Y-chromosome STRs in a population of Northern Greeks. Forensic Sci Int: Genet 4:e21-22.

Lawlor A. 1998. The Eleutherian Adventurers. J Bahamas Hist Soc 20:4-9.

Luis JR, Rowold DJ, Regueiro M, Caeiro B, Cinnioğlu C, Roseman C, Underhill PA, Cavalli-Sforza LL, Herrera RJ. 2004. The Levant versus the Horn of Africa: evidence for bidirectional corridors of human migrations. Am J Hum Genet 74:532-544. 
Malhi RS, Gonzalez-Oliver A, Schroeder KB, Kemp BM, Greenberg JA, Dobrowski SZ, Smith DG, Resendez A, Karafet T, Hammer M, Zegura S, Brovko T. 2008. Distribution of Y chromosomes among native North Americans: a study of Athapaskan population history. Am J Phys Anthropol 137:412-424.

Marjanovic D, Fornarino S, Montagna S, Primorac D, Hadziselimovic R, Vidovic S, Pojskic N, Battaglia V, Achilli A, Drobnic K, Andjelinovic S, Torroni A, SantachiaraBenerecetti AS, Semino O. 2005. The peopling of modern Bosnia-Herzegovina: Ychromosome haplogroups in the three main ethnic groups. Ann Hum Genet 69:757-763.

Martín P, García-Hirschfeld J, García O, Gusmão L, García P, Albarrán C, Sancho M, Alonso A. 2004. A Spanish population study of 17 Y-chromosome STR loci. Forensic Sci Int 139:231-235.

Martínez-Cruzado JC, Toro-Labrador G, Viera-Vera J, Rivera-Vega MY, Startek J, Latorre-Esteves M, Román-Colón A, Rivera-Torres R, Navarro-Millán IY, GómezSánchez E, Caro-González HY, Valencia-Rivera P. 2005. Reconstructing the population history of Puerto Rico by means of mtDNA phylogeographic analysis. Am J Phys Anthropol 128:131-155.

McCartney DM. 2004. Bahamian culture and factors which impact upon it. Pittsburg, PA: Dorrance Publishing Co., Inc.

Mendizabal I, Sandoval K, Berniell-Lee G, Calafell F, Salas A, Martínez-Fuentes A, Comas D. 2008. Genetic origin, admixture, and asymmetry in maternal and paternal human lineages in Cuba. BMC Genet 8:213.

Miller WH. 1945. The colonization of the Bahamas, 1647-1670. The William and Mary Quarterly $3^{\text {rd }}$ Ser 2:33-46.

Mirabal S, Varljen T, Gayden T, Regueiro M, Vujovic S, Popovic D, Djuric M, Stojkovic O, Herrera RJ. 2010. Human Y-chromosome short tandem repeats: a tale of acculturation and migrations as mechanisms for the diffusion of agriculture in the Balkan Peninsula. Am J Phys Anthropol 142:380-390.

Myres NM, Rootsi S, Lin AA, Järve M, King RJ, Kutuev I, Cabrera VM, Khusnutdinova EK, Pshenichnov A, Yunusbayev B, Balanovsky O, Balanovska E, Rudan P, Baldovic M, Herrera RJ, Chiaroni J, Di Cristofaro J, Villems R, Kivisild T, Underhill PA. 2011. A major Y-chromosome haplogroup R1b Holocene era founder effect in Central and Western Europe. Eur J Hum Genet 19:95-101.

Nuñez C, Baeta M, Sosa C, Casalod Y, Ge J, Budowle B, Martínez-Jarreta B. 2010. Reconstructing the population history of Nicaragua by means of mtDNA, Y-chromosome STRs, and autosomal STR markers. Am J Phys Anthropol 143:591-600. 
Onofri V, Alessandrini F, Turchi C, Fraternale B, Buscemi L, Pesaresi M, Tagliabracci A. 2007. Y-chromosome genetic structure in sub-Apennine populations of Central Italy by SNP and STR analysis. Int J Legal Med 121:234-237.

Plaza S, Salas A, Calafell F, Corte-Real F, Bertranpetit J, Carracedo Á, Comas D. 2004. Insights into the western Bantu dispersal: mtDNA lineage analysis in Angola. Hum Genet 115:439-447.

Pepin J. 2005. From the Old World to the New World: an ecologic study of population susceptibility to HIV infection. Trop Med Int Health 10:627-639.

Pereira L, Macaulay V, Torroni A, Scozzari R, Prata MJ, Amorim A. 2001. Prehistoric and historic traces in the mtDNA of Mozambique: insights into the Bantu expansions and the slave trade. Am J Hum Genet 65:439-458.

Petrejčíková E, Soták M, Bernasovská J, Bernasovský I, Sovičová A, Bôžiková A, Boroňová I, Gabriková D, Švíčková P, Mačeková S, Čverhová V. 2010. The genetic structure of the Slovak population revealed by Y-chromosome polymorphisms. Anthropol Sci 118:23-30.

Raymond M, Rousset F. 1995. GENEPOP (Ver. 1.2): a population genetics software for exact test and ecumenicism. J Hered 86:248-249.

Rębała K, Szczerkowska Z. 2005. Polish population study on Y chromosome haplotypes defined by 18 STR loci. Int J Legal Med 119:303-305.

Richardson, BC. 1989. Caribbean migrations, 1838-1985. In: Knight, FW, Palmer, CA, editors. The modern Caribbean. Chapel Hill, NC: University of North Carolina Press. p 203-228.

Rootsi S, Magri C, Kivisild T, Benuzzi G, Help H, Bermisheva M, Kutuev I, Barać L, Pericić M, Balanovsky O, Pshenichnov A, Dion D, Grobei M, Zhivotovsky LA, Battaglia V, Achilli A, Al-Zahery N, Parik J, King R, Cinnioğlu C, Khusnutdinova E, Rudan P, Balanovska E, Scheffrahn W, Simonescu M, Brehm A, Goncalves R, Rosa A, Moisan JP, Chaventre A, Ferak V, Füredi S, Oefner PJ, Shen P, Beckman L, Mikerezi I, Terzić R, Primorac D, Cambon-Thomsen A, Krumina A, Torroni A, Underhill PA, SantachiaraBenerecetti AS, Villems R, Semino O. 2004. Phylogeography of Y-chromosome haplogroup I reveals distinct domains of prehistoric gene flow in Europe. Am J Hum Genet 75:128-137.

Rosa A, Ornelas C, Jobling MA, Brehm A, Villems R. 2007. Y-chromosomal diversity in the population of Guinea-Bissau: a multiethnic perspective. BMC Evol Biol 7:124.

Saunders G. 1983. The Loyalists: General influences. J Bahamas Hist Soc 5:3-10. 
Saunders G.1985. Slavery in the Bahamas 1648-1838. Nassau, Bahamas: The Nassau Guardian.

Saunders G. 1990. Life in New Providence in the early eighteenth century. J Bahamas Hist Soc 12:9-14.

Saunders G. 1991. The early settlers in the Bahamas. J Bahamas Hist Soc 13:13-17.

Saunders G. 1995. Aspects of traditional African-Bahamian culture in the later $19^{\text {th }}$ and early $20^{\text {th }}$ century. J Bahamas Hist Soc 17:2-10.

Saunders G. 1996. Social Life in the Bahamas 1880s-1920s.Nassau, Bahamas: Rosebud Ltd.

Saunders G. 2003a. The Bahamas. In: West-Durán A, editor. African Caribbeans: a reference guide. Westport, CT: Greenwood Press. p 13-28.

Saunders G. 2003b. Bahamian society after emancipation. Kingston, Jamaica: Ian Randle Publishers.

Semino O, Santachiara-Benerecetti AS, Falaschi F, Cavalli-Sforza LL, Underhill PA. 2002. Ethiopians and Khoisan share the deepest clades of the human Y-chromosome phylogeny. Am J Hum Genet 70:265-268.

Semino O, Magri C, Benuzzi G, Lin AA, Al-Zahery N, Battaglia V, Maccioni L, Triantaphyllidis C, Shen P, Oefner PJ, Zhivotovsky LA, King R, Torroni A, CavalliSforza LL, Underhill PA, Santachiara-Benerecetti AS. 2004. Origin, diffusion, and differentiation of Y-chromosome haplogroups $\mathrm{E}$ and $\mathrm{J}$ : inferences on the Neolithization of Europe and later migratory events in the Mediterranean area. Am J Hum Genet 74:1023-1034.

Sharer CJ. 1955. The population growth of the Bahama Islands. Dissertation: University of Michigan.

Simms TM, Garcia C, Mirabal S, McCartney Q, Herrera RJ. 2008. The genetic legacy of the Transatlantic Slave Trade in the island of New Providence. Forensic Sci Int: Genet 2:310-317.

Simms TM, Rodriguez CE, Rodriguez R, Herrera RJ. 2010. The genetic structure of populations from Haiti and Jamaica reflect divergent demographic histories. Am J Phys Anthropol 142:49-66.

Simms TM, Barrett DA, McCartney Q, Herrera RJ. 2011. Divergent genetic strata in five Bahamian islands. In Press Forensic Science International: Genetics. 
Sims LM, Garvey D, Ballantyne J. 2007. Sub-Populations within the major European and African derived haplogroups R1b3 and E3a are differentiated by previously phylogenetically undefined Y-SNPs. Hum Mutat 2007 28:97.

Spiroski M, Arsov T, Krüger C, Willuweit S, Roewer L. 2005. Y-chromosomal STR haplotypes in Macedonian population samples. Forensic Sci Int 148:69-73.

SPSS for Windows, Rel. 18.0.2. 2010. SPSS Inc, Chicago, IL, USA

Stanciu F, Cuţăr V, Pîrlea S, Stoian V, Stoian IM, Sevastre O, Popescu OR. 2010. Population data for Y-chromosome haplotypes defined by 17 STRs in South-East Romania. Leg Med 12:259-64.

Thomas H. 1998. The slave trade - the history of the Atlantic slave trade: 1440-1870. London: Macmillan Publishers Ltd.

Tinker KL. 1998. Perspectives on West Indian migration to the Bahamas: pre-Columbian to Bahamian independence in 1973. Dissertation: Florida State University.

Tishkoff SA, Gonder MK, Henn BM, Mortensen H, Knight A, Gignoux C, Fernandopulle N, Lema G, Nyambo TB, Ramakrishnan U, Reed FA, Mountain JL. 2007. History of click-speaking populations of Africa inferred from mtDNA and Y chromosome genetic variation. Mol Biol Evol 24:2180-95.

Toro-Labrador G, Wever OR, Martínez-Cruzado JC. 2003. Mitochondrial DNA analysis in Aruba: strong maternal ancestry of closely related Amerindians and implications for the peopling of Northwestern Venezuela. Caribb J Sci 39:11-22.

Underhill PA, Shen P, Lin AA, Jin L, Passarino G, Yang WH, Kauffman E, Bonné-Tamir B, Bertranpetit J, Francalacci P, Ibrahim M, Jenkins T, Kidd JR, Mehdi SQ, Seielstad MT, Wells RS, Piazza A, Davis RW, Feldman MW, Cavalli-Sforza LL, Oefner PJ. 2000. $\mathrm{Y}$ chromosome sequence variation and the history of human populations. Nat Genet 26:358-361.

Underhill PA, Passarino G, Lin AA, Shen P, Mirazon Lahr M, Foley RA, Oefner PJ, Cavalli-Sforza LL. 2001. The phylogeography of Y chromosome binary haplotypes and the origins of modern human populations. Ann Hum Genet 65:43-62.

Underhill PA, Myres NM, Rootsi S, Metspalu M, Zhivotovsky LA, King RJ, Lin AA, Chow CT, Semino O, Battaglia V, Kutuev I, Järve M, Chaubey G, Ayub Q, Mohyuddin A, Mehdi SQ, Sengupta S, Rogaev EI, Khusnutdinova EK, Pshenichnov A, Balanovsky O, Balanovska E, Jeran N, Augustin DH, Baldovic M, Herrera RJ, Thangaraj K, Singh V, Singh L, Majumder P, Rudan P, Primorac D, Villems R, Kivisild T. 2010. Separating the post-Glacial coancestry of European and Asian Y chromosomes within haplogroup R1a. Eur J Hum Genet 18:479-484. 
Vallone PM, Butler JM. 2004. Y-SNP typing of U.S. African American and Caucasian samples using allele-specific hybridization and primer extension. J Forensic Sci 49:723732.

Veeramah KR, Connell BA, Pour NA, Powell A, Plaster CA, Zeitlyn D, Mendell NR, Weale ME, Bradman N, Thomas MG. 2010. Little genetic differentiation as assessed by uniparental markers in the presence of substantial language variation in peoples of the Cross River region of Nigeria. BMC Evol Biol 10:92

Wilkie LA. 1993. Continuities in African naming practices among slaves of Wade's Green Plantation, North Caicos. J Bahamas Hist Soc 15:32-37.

Williams P. 1983. The Loyalists and their settlements. J Bahamas Hist Soc 5:11-15.

Wood ET, Stover DA, Ehret C, Destro-Bisol G, Spedini G, Mcleod H, Louie L, Bamshad M, Strassmann BI, Soodyall H, Hammer MF. 2005. Contrasting patterns of Y

chromosome and mtDNA variation in Africa: evidence for sex-biased demographic processes. Eur J Hum Genet 13:867-76.

Zalloua PA, Xue Y, Khalife J, Makhoul N, Debiane L, Platt DE, Royyuru AK, Herrera RJ, Soria Hernanz DF, Blue-Smith J, Wells RS, Comas D, Bertranpetit J, Tyler-Smith C, The Genographic Consortium. 2008. Y-chromosomal diversity in Lebanon is structured by recent historical events. Am J Hum Genet 82:873-882.

Zegura SL, Karafet TM, Zhivotovsky LA, Hammer MF. 2004. High-resolution SNPs and microsatellite haplotypes point to a single, recent entry of Native American Y chromosomes into the Americas. Mol Biol Evol 21:164-175. 


\section{Appendix V}

Fig. 1: Hierarchical phylogenetic relationships and frequencies (\%) of the 44 paternal haplogroups observed in the six Bahamian islands analyzed in the current investigation. M75, P147, P177, P123 and P143 (in italics) were not genotyped but were included for phylogenetic context.
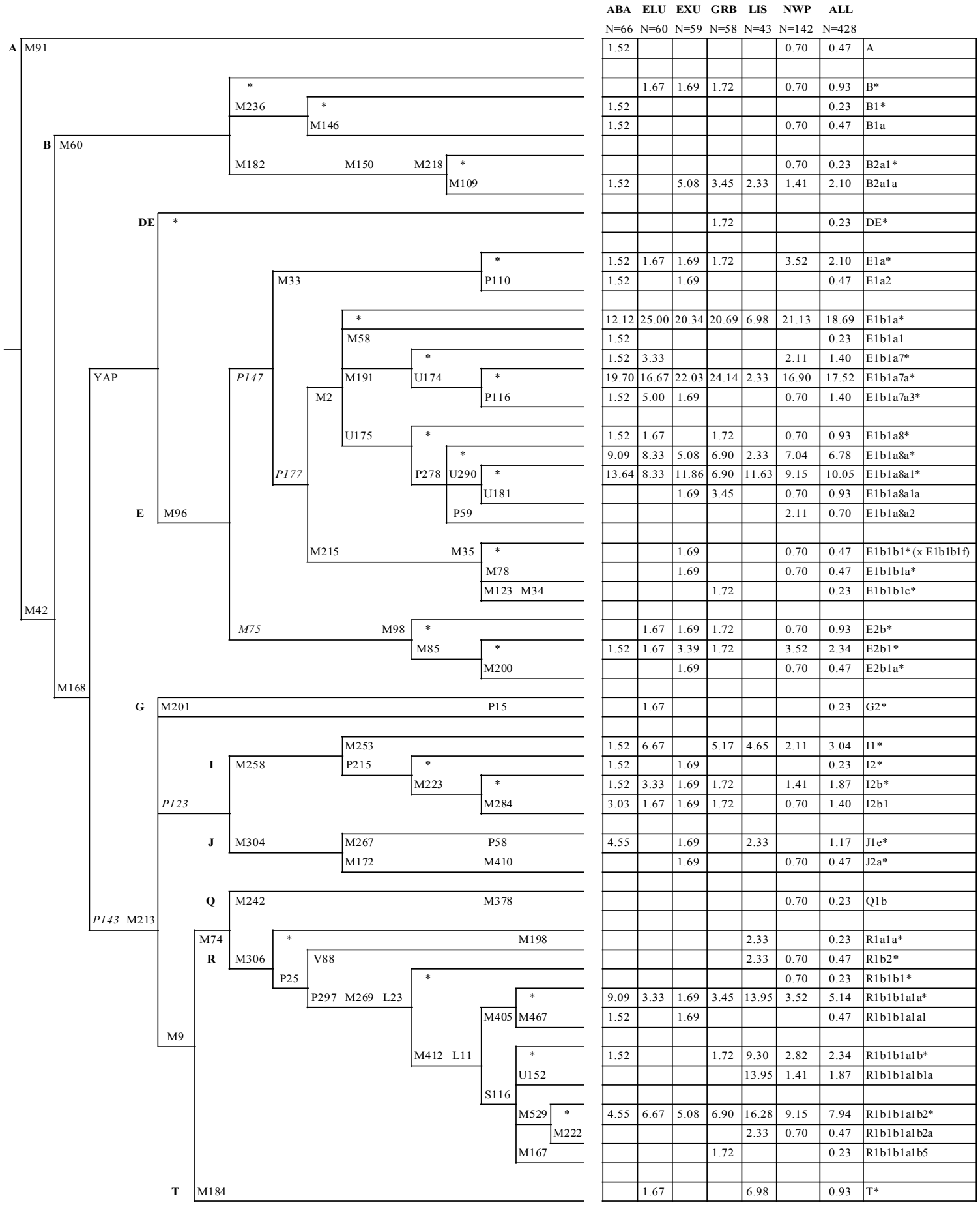
Fig. 2A: Global Y-chromosome frequency distribution at the level of the major haplogroups (A-T)

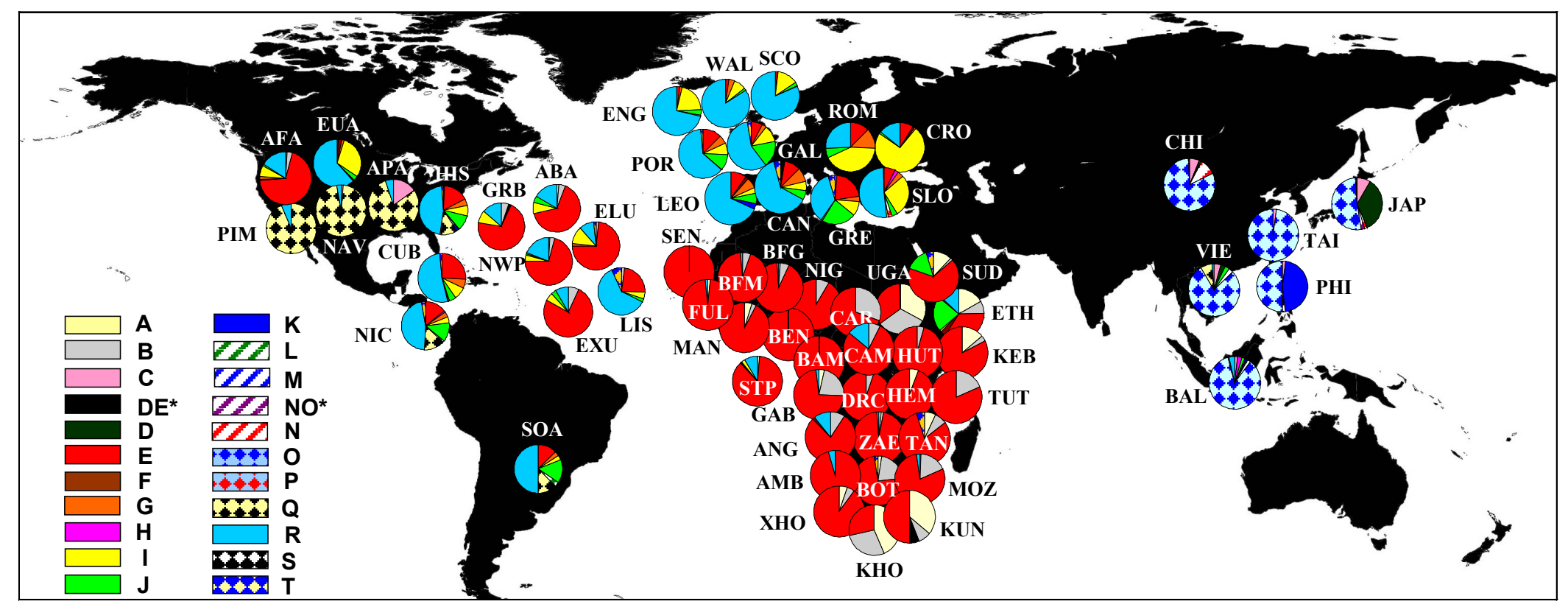


Fig. 2B: Frequency distributions of Y-chromosome E sub-haplogroups within the Bahamian archipelago

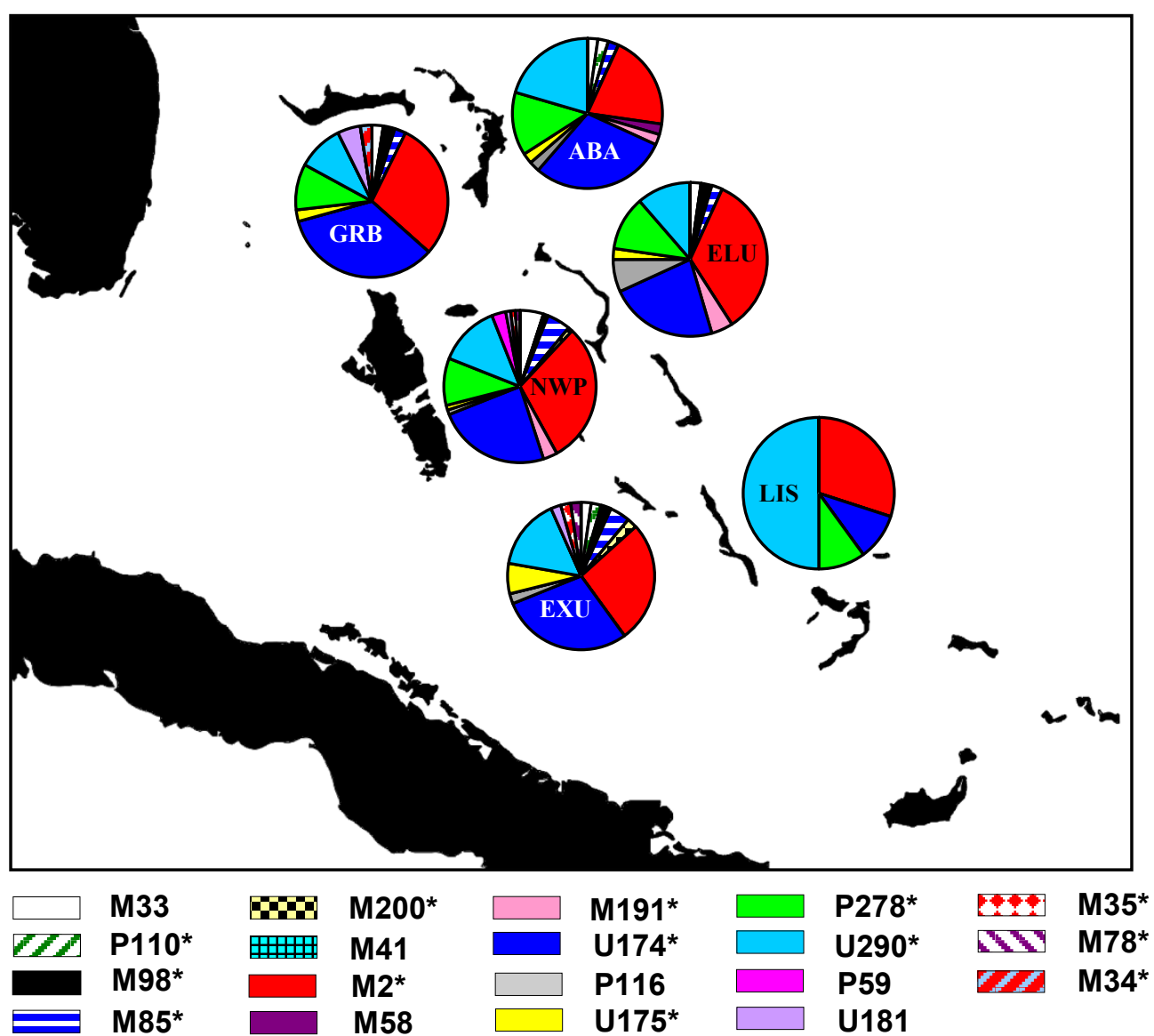


Fig. 3: Multidimensional Scaling plot based on Y-haplogroup frequency data of the populations listed in Table 1

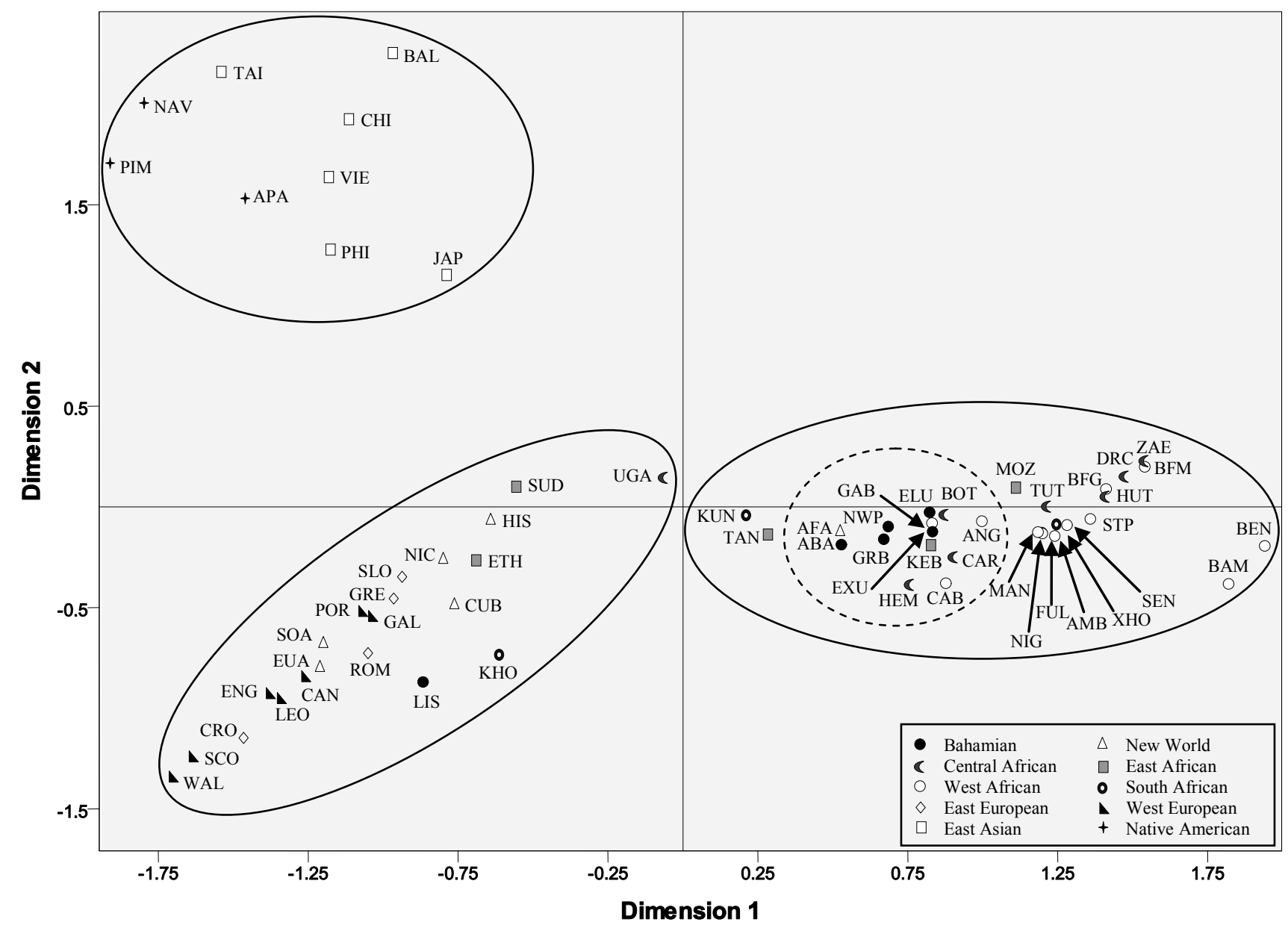


Fig. 4A: Network projection based on the Y-STR profiles within sub-haplogroup E1b1a7a-U174

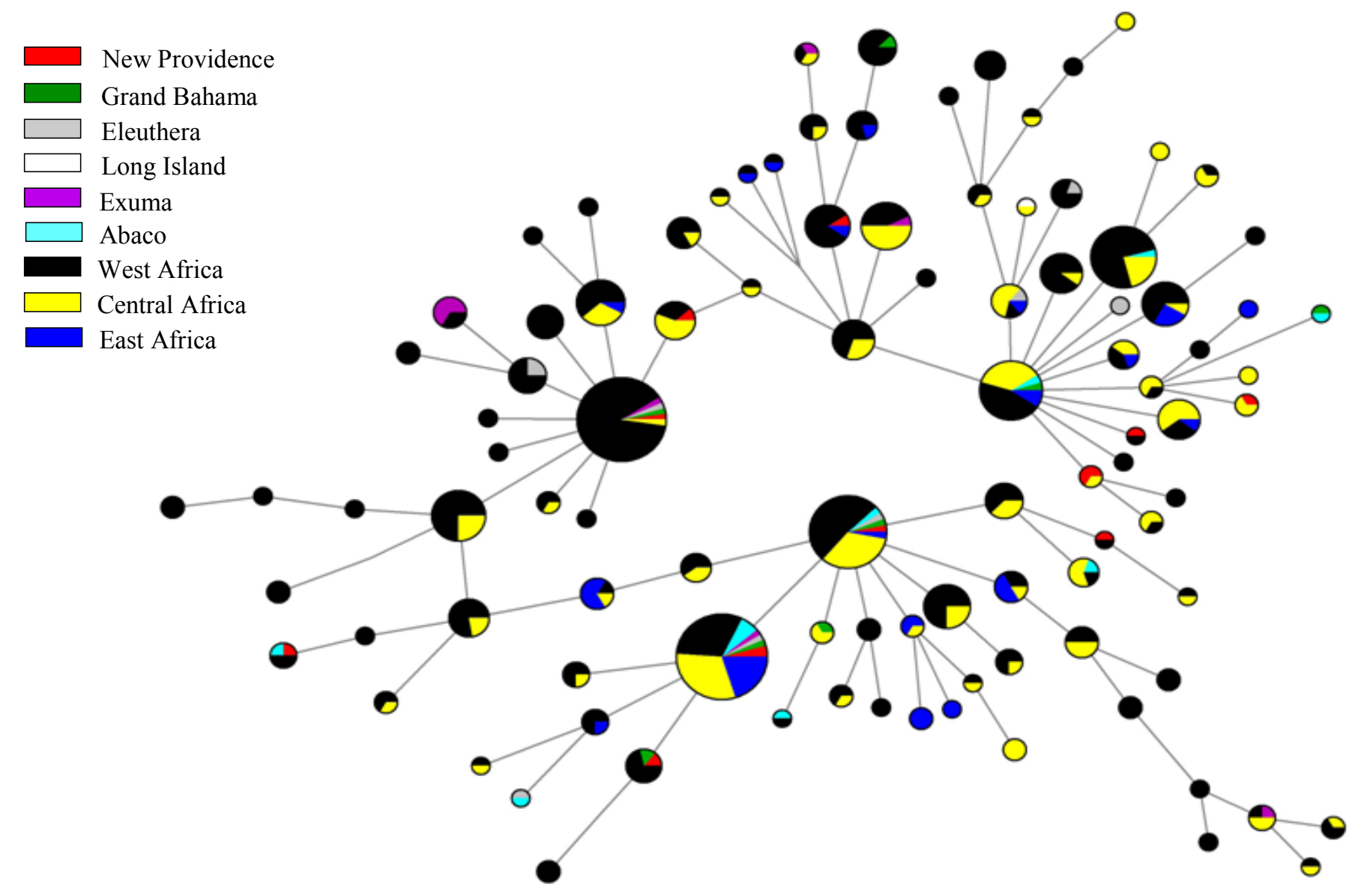


Fig. 4B: Network projection based on the Y-STR profiles within sub-haplogroup E1b1a8-U175

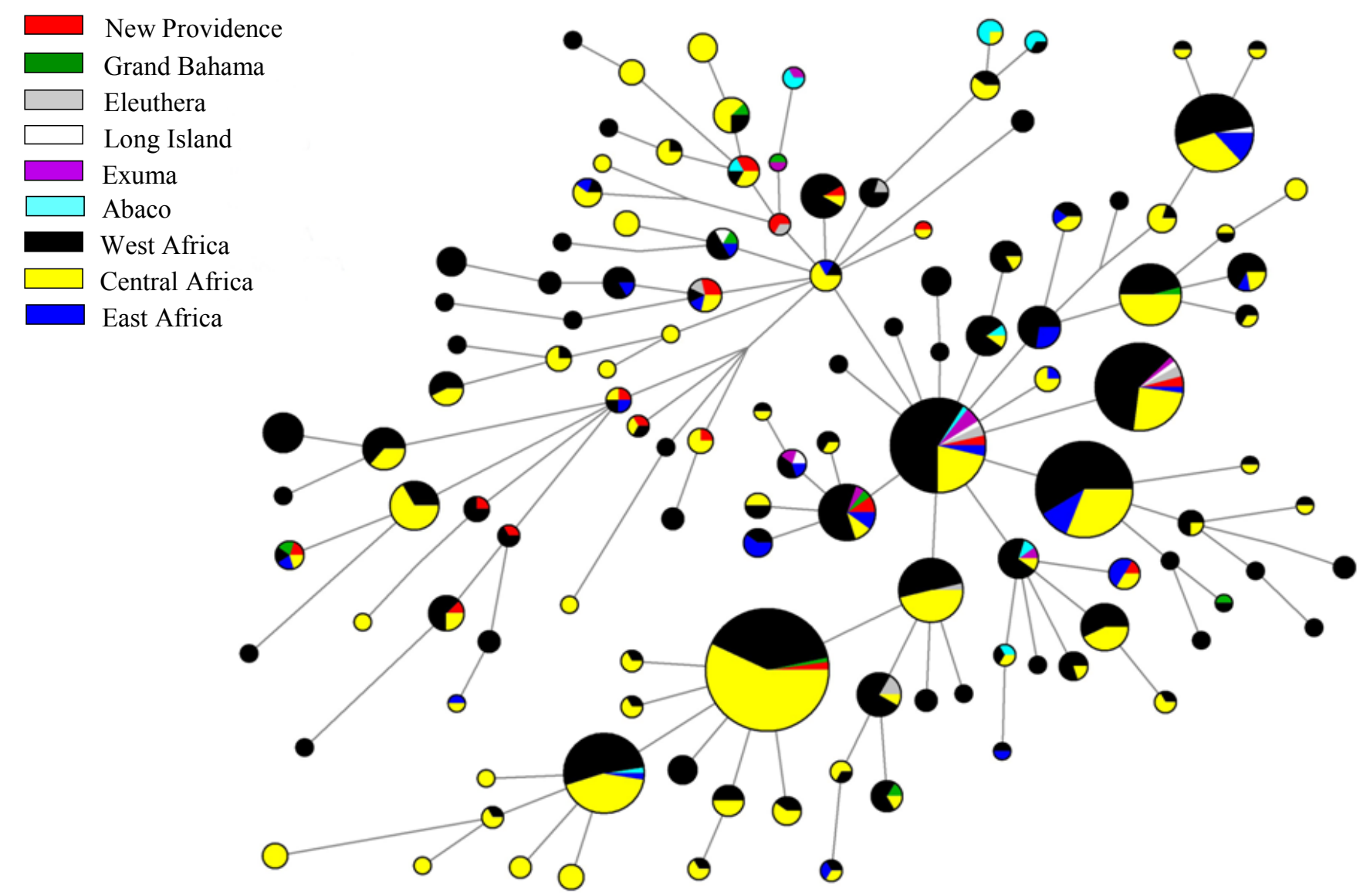


Fig. 5: Network projection based on the Y-STR profiles within sub-haplogroup R1b1b1-M269
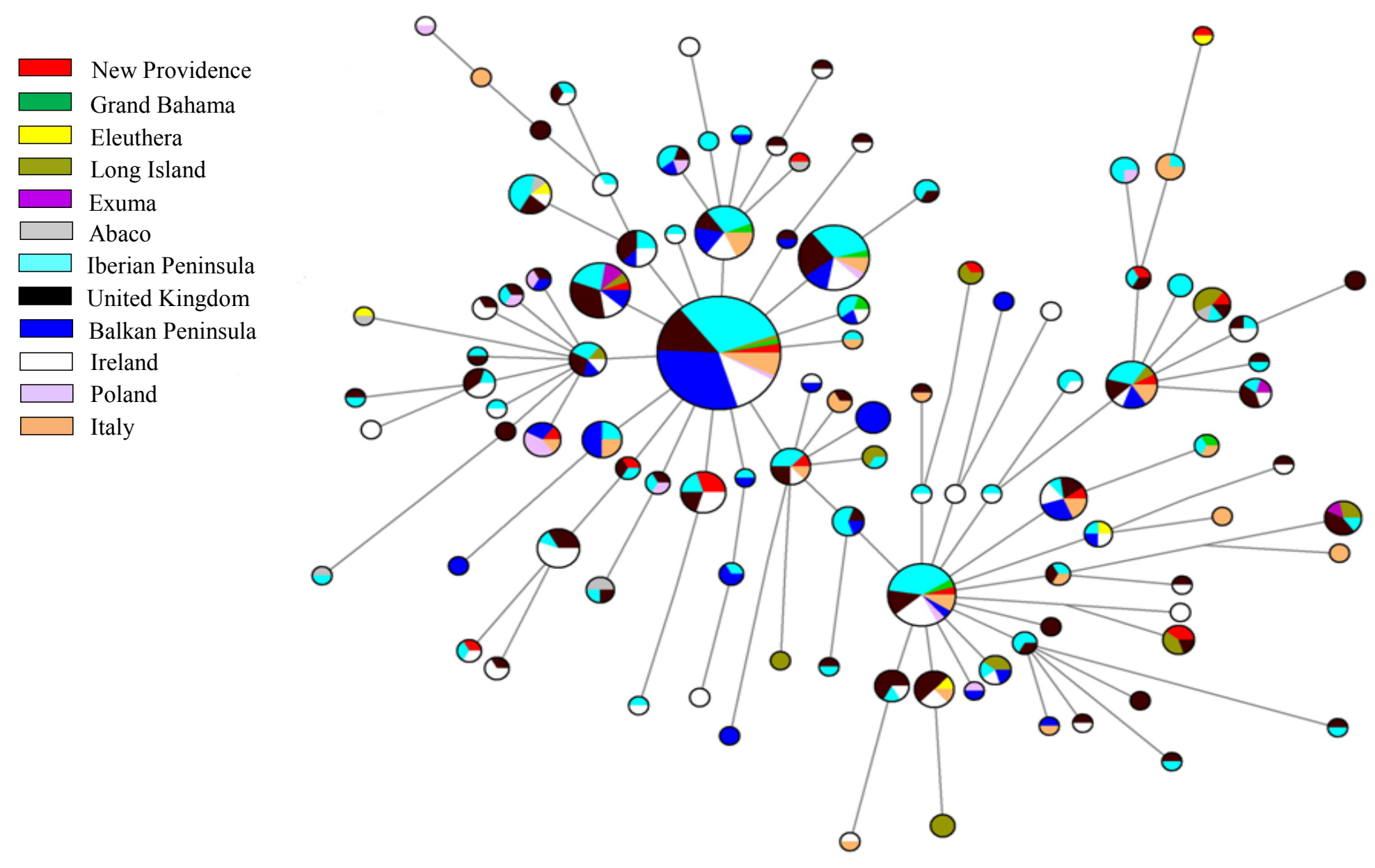
Fig. 6A: Multidimensional Scaling plot based on Y-STR allelic frequency data for individuals possessing haplogroup E1b1a7a-U174 derivatives

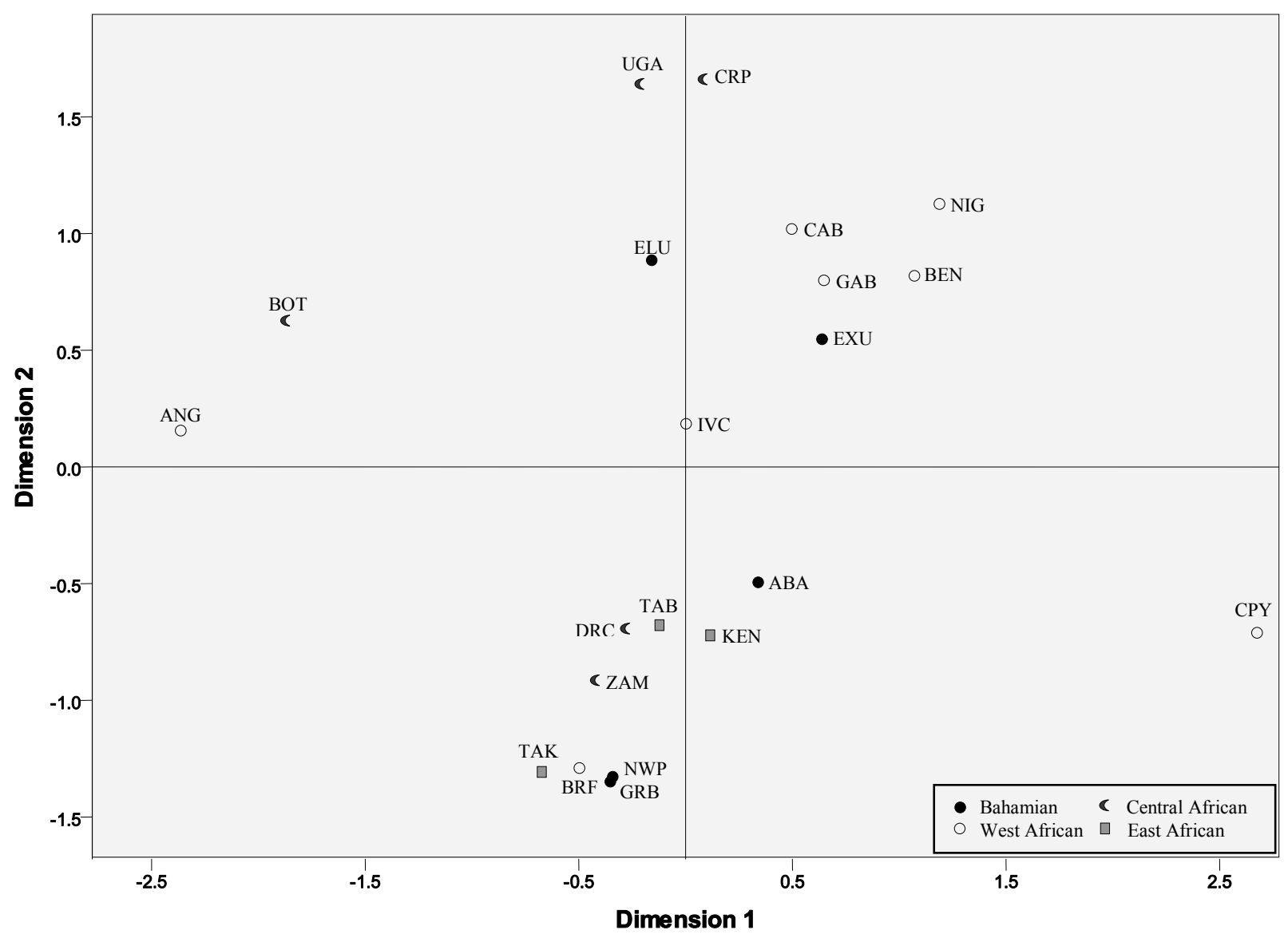


Fig. 6B: Multidimensional Scaling plot based on Y-STR allelic frequency data for individuals possessing haplogroup E1b1a8-U175 derivatives

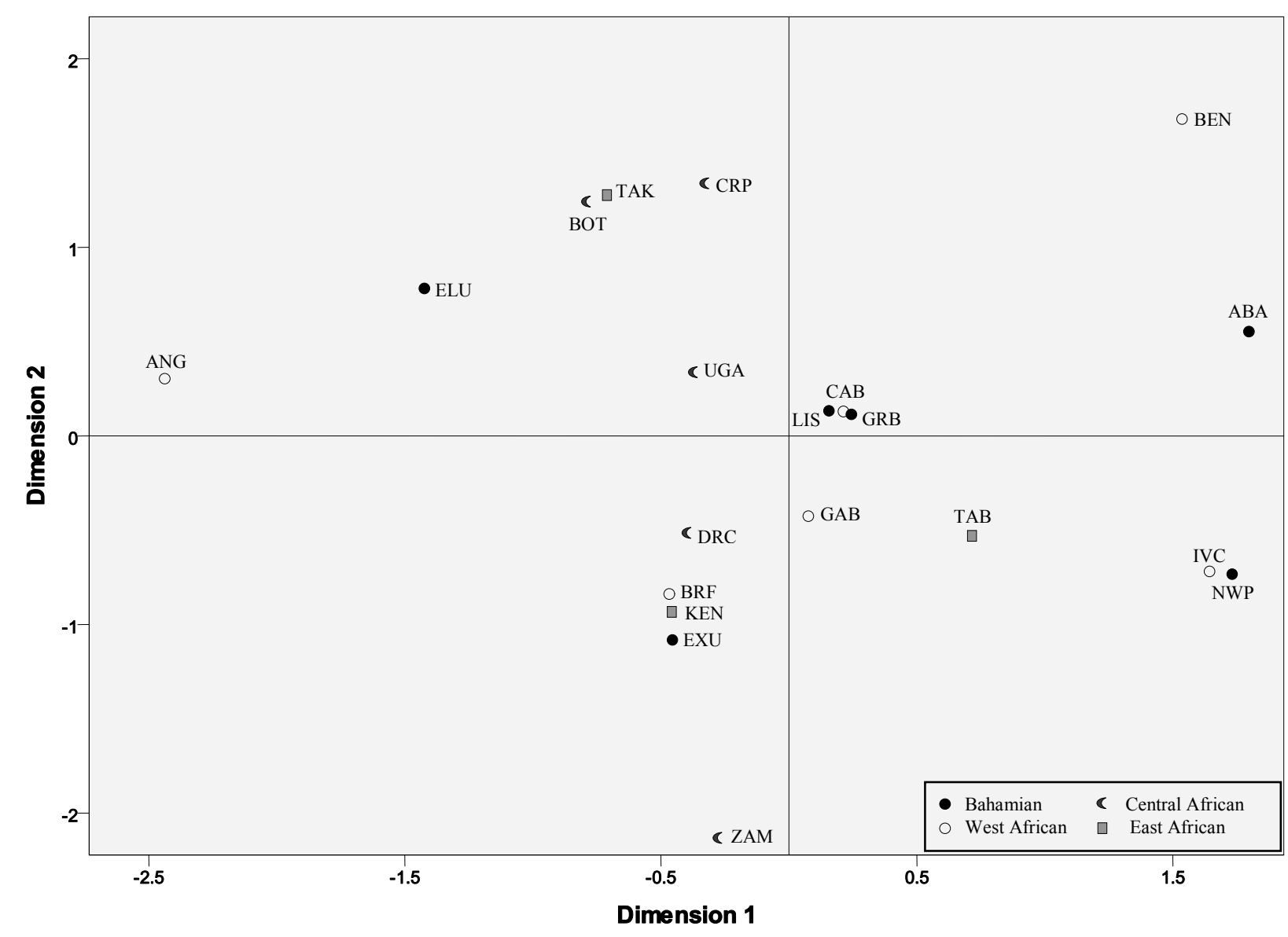




\begin{tabular}{|c|c|c|c|c|}
\hline $\begin{array}{l}\text { Biogeographical } \\
\text { origin }\end{array}$ & Population & $\begin{array}{l}\text { Sample } \\
\text { Size }\end{array}$ & Abbreviation & Reference \\
\hline \multicolumn{5}{|l|}{ NEW WORLD } \\
\hline \multicolumn{5}{|c|}{ Bahamian } \\
\hline & Abaco & 66 & $\mathrm{ABA}$ & Present study \\
\hline & Eleuthera & 60 & ELU & Present study \\
\hline & Exuma & 59 & EXU & Present study \\
\hline & Grand Bahama & 58 & GRB & Present study \\
\hline & Long Island & 43 & LIS & Present study \\
\hline & New Providence & 142 & NWP & Present study \\
\hline \multicolumn{5}{|c|}{ Latin American } \\
\hline & Cuba & 132 & CUB & Mendizabal et al., 2008 \\
\hline & Nicaragua & 165 & NIC & Nuñez et al., 2010 \\
\hline & Argentina (South) & 32 & SOA & Corach et al., 2010 \\
\hline \multicolumn{5}{|c|}{ United States } \\
\hline & Hispanic Americans & 479 & HIS & Hammer et al., 2006 \\
\hline & European Americans & 125 & EUA & Sims et al., 2007 \\
\hline & African Americans & 118 & AFA & Sims et al., 2007 \\
\hline \multicolumn{5}{|l|}{ EUROPEAN } \\
\hline \multicolumn{5}{|c|}{ West } \\
\hline & Cantabria & 70 & CAN & Flores et al, 2004 \\
\hline & Leon & 60 & LEO & Flores et al, 2004 \\
\hline & Galicia & 292 & GAL & Brion et al., 2004 \\
\hline & England & 1344 & ENG & Campbell et al., 2007 \\
\hline & Portugal & 657 & POR & Beleza et al., 2006 \\
\hline & Scotland & 775 & SCO & Campbell et al., 2007 \\
\hline & Wales & 178 & WAL & Campbell et al., 2007 \\
\hline \multicolumn{5}{|c|}{ East } \\
\hline & Croatia & 90 & $\mathrm{CRO}$ & Marjanovic et al., 2005 \\
\hline & Greece & 92 & GRE & Battaglia et al., 2009 \\
\hline & Romania & 31 & ROM & Bosch et al., 2006 \\
\hline & Slovakia & 250 & SLO & Petrejčíková et al., 2010 \\
\hline \multicolumn{5}{|l|}{ EAST ASIAN } \\
\hline & Bali (Indonesia) & 641 & BAL & Karafet et al., 2010 \\
\hline & China (Han) & 165 & $\mathrm{CHI}$ & Karafet et al., 2010 \\
\hline & Japan & 259 & JAP & Hammer et al.,2006 \\
\hline & Philippines & 48 & PHI & Karafet et al., 2010 \\
\hline & Taiwan & 48 & TAI & Karafet et al., 2010 \\
\hline & Veitnam & 70 & VIE & Karafet et al., 2010 \\
\hline
\end{tabular}

Table 1: Populations utilized in Y-SNP analyses 


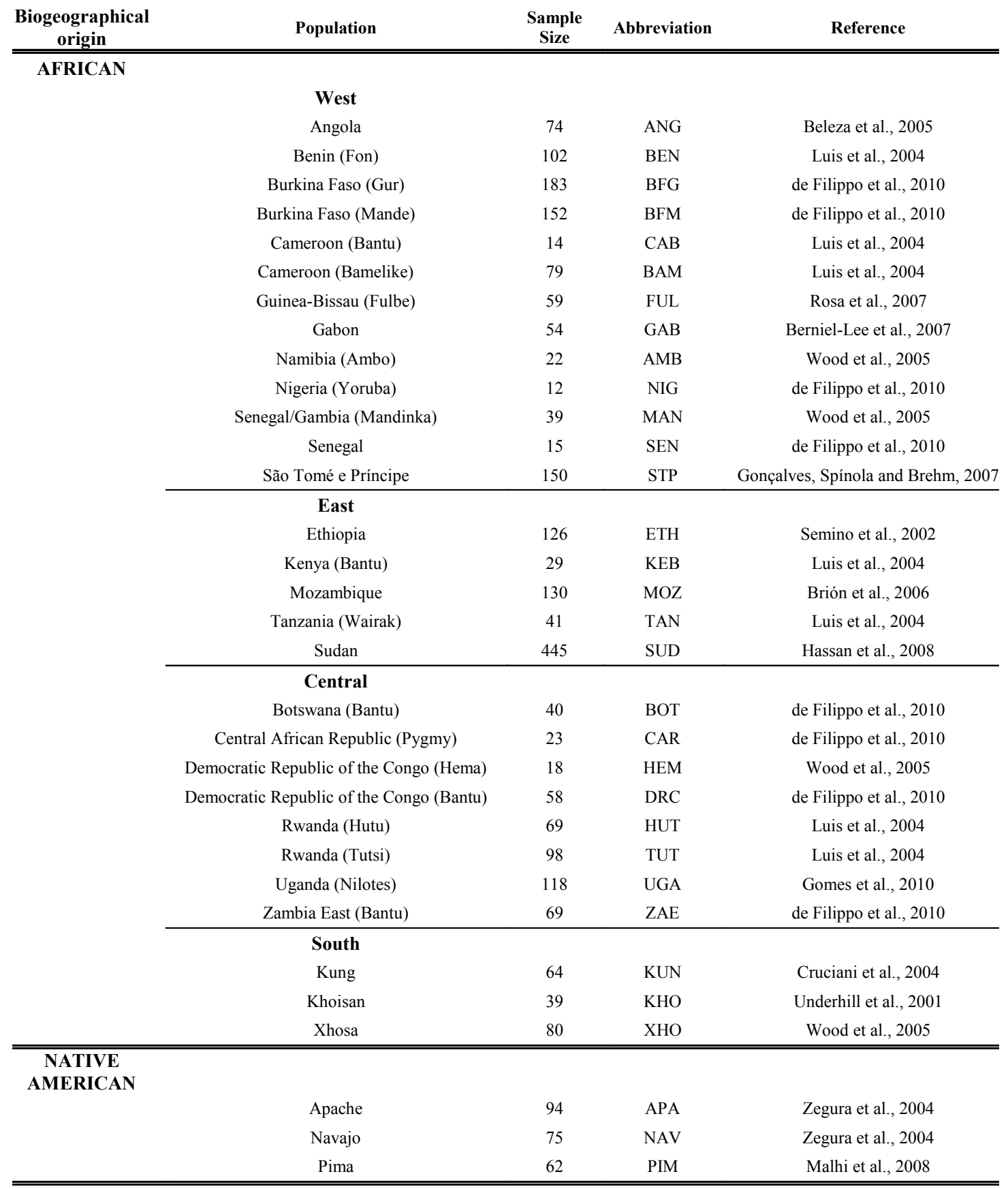

Table 1: Populations utilized in Y-SNP analyses (Cont'd) 


\begin{tabular}{|c|c|c|c|c|c|c|c|c|c|c|c|c|c|c|c|}
\hline \multirow{3}{*}{\multicolumn{2}{|c|}{ Population }} & \multirow[b]{3}{*}{$\mathbf{N}$} & \multicolumn{13}{|c|}{ Frequency of Haplogroup (\%) } \\
\hline & & & \multicolumn{7}{|c|}{ E1b1a7 } & \multicolumn{6}{|c|}{ E1b1a8 } \\
\hline & & & M191 & U174 & P9.2 & P115 & P116 & P113 & Total & U175 & P278 & U290 & P59 & U181 & Total \\
\hline \multirow{6}{*}{ 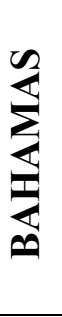 } & Abaco & 66 & 1.52 & 19.70 & - & - & 1.52 & - & 22.73 & 1.52 & 9.09 & 13.64 & - & - & 24.24 \\
\hline & Eleuthera & 60 & 3.33 & 16.67 & - & - & 5.00 & - & 25.00 & 1.67 & 8.33 & 8.33 & - & - & 18.33 \\
\hline & Exuma & 59 & - & 22.03 & - & - & 1.69 & - & 23.73 & - & 5.08 & 11.86 & - & 1.69 & 18.64 \\
\hline & Grand Bahama & 58 & - & 24.14 & - & - & - & - & 24.14 & 1.72 & 6.90 & 6.90 & - & 3.45 & 18.97 \\
\hline & Long Island & 43 & - & 2.33 & - & - & - & - & 2.33 & - & 2.33 & 11.63 & - & - & 13.95 \\
\hline & New Providence & 142 & 2.11 & 16.90 & - & - & 0.70 & - & 19.72 & 0.70 & 7.04 & 9.15 & 2.11 & 0.70 & 19.72 \\
\hline \multirow{7}{*}{$\frac{\mho}{\tilde{U}}$} & West & & & & & & & & & & & & & & \\
\hline & Benin (Fon) & 102 & 2.94 & 54.90 & - & - & - & - & $\mathbf{5 7 . 8 4}$ & 0.98 & 15.69 & 10.78 & - & - & 11.76 \\
\hline & Cameroon (Bantu) & 14 & - & 14.29 & - & - & 7.14 & - & 21.43 & - & - & 42.86 & - & - & 42.86 \\
\hline & Central & & & & & & & & & & & & & & \\
\hline & Rwanda (Hutu) & 69 & 23.19 & 14.49 & - & - & 13.04 & - & 50.72 & - & 1.45 & 1.45 & - & - & 2.90 \\
\hline & East & & & & & & & & & & & & & & \\
\hline & Kenya (Bantu) & 29 & - & 31.03 & - & - & - & - & 31.03 & - & - & 13.79 & 3.45 & - & 17.24 \\
\hline
\end{tabular}

- indicates that the haplogroup was not detected

Table 2: Frequencies of E1b1a7 and E1b1a8 sub-haplogroups in the Bahamian and continental African populations 
BAHAMAS

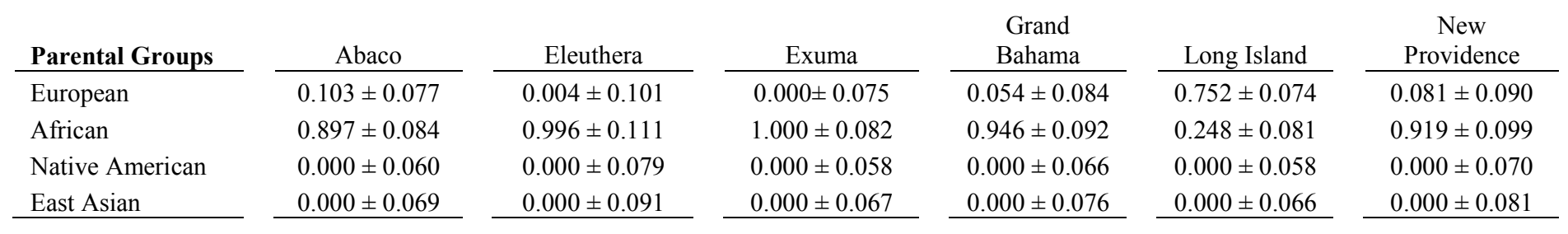

NEW WORLD

\begin{tabular}{|c|c|c|c|c|c|c|}
\hline Parental Groups & Cuba & Nicaragua & $\begin{array}{c}\text { South } \\
\text { Argentina } \\
\end{array}$ & $\begin{array}{l}\text { European } \\
\text { American }\end{array}$ & $\begin{array}{l}\text { Hispanic } \\
\text { American } \\
\end{array}$ & $\begin{array}{c}\text { African } \\
\text { American } \\
\end{array}$ \\
\hline European & $0.765 \pm 0.041$ & $0.686 \pm 0.052$ & $0.749 \pm 0.073$ & $0.974 \pm 0.062$ & $0.673 \pm 0.032$ & $0.084 \pm 0.078$ \\
\hline African & $0.218 \pm 0.045$ & $0.131 \pm 0.057$ & $0.071 \pm 0.080$ & $0.000 \pm 0.068$ & $0.165 \pm 0.035$ & $0.916 \pm 0.085$ \\
\hline Native American & $0.001 \pm 0.032$ & $0.165 \pm 0.041$ & $0.154 \pm 0.057$ & $0.012 \pm 0.049$ & $0.132 \pm 0.025$ & $0.000 \pm 0.061$ \\
\hline East Asian & $0.017 \pm 0.037$ & $0.018 \pm 0.047$ & $0.026 \pm 0.066$ & $0.014 \pm 0.056$ & $0.029 \pm 0.029$ & $0.000 \pm 0.070$ \\
\hline
\end{tabular}

Table 3: Admixture proportions generated for the Bahamian and New World populations 


\begin{tabular}{|c|c|c|c|c|c|c|c|c|c|c|c|c|c|c|c|c|c|}
\hline Sample & DYS456 & DYS389I & DYS390 & DYS389II & DYS458 & DYS19 & DYS385a & DYS385b & DYS393 & DYS391 & DYS439 & DYS635 & DYS392 & GATA_H4 & DYS437 & DYS438 & DYS448 \\
\hline NWP3 & 15 & 13 & 22 & 30 & 16 & 15 & 17 & 19 & 14 & 10 & 12 & 22 & 11 & 11 & 14 & 11 & 21 \\
\hline NWP4 & 15 & 13 & 21 & 30 & 16 & 17 & 18 & 19 & 14 & 10 & 12 & 21 & 11 & 11 & 14 & 11 & 21 \\
\hline NWP6 & 15 & 13 & 21 & 30 & 18 & 16 & 15 & 19 & 14 & 10 & 11 & 21 & 12 & 11 & 14 & 11 & 20 \\
\hline NWP11 & 16 & 14 & 21 & 31 & 15 & 17 & 18 & 19 & 15 & 10 & 11 & 21 & 11 & 11 & 14 & 11 & 21 \\
\hline NWP24 & 17 & 14 & 21 & 31 & 16 & 17 & 17 & 17 & 14 & 10 & 11 & 20 & 11 & 11 & 14 & 11 & 21 \\
\hline NWP29 & 16 & 12 & 21 & 29 & 15 & 16 & 17 & 19 & 15 & 10 & 12 & 21 & 11 & 11 & 14 & 11 & 21 \\
\hline NWP32 & 14 & 13 & 20 & 31 & 15 & 16 & 15 & 18 & 14 & 10 & 13 & 21 & 11 & 11 & 14 & 11 & 21 \\
\hline NWP39 & 16 & 12 & 21 & 29 & 15 & 16 & 17 & 19 & 15 & 10 & 12 & 21 & 11 & 11 & 14 & 11 & 20 \\
\hline NWP48 & 15 & 13 & 25 & 29 & 15 & 14 & 15 & 15 & 13 & 10 & 11 & 24 & 13 & 12 & 15 & 12 & 18 \\
\hline NWP51 & 16 & 13 & 21 & 30 & 16 & 16 & 17 & 17 & 15 & 11 & 12 & 22 & 11 & 11 & 14 & 11 & 21 \\
\hline NWP53 & 15 & 13 & 21 & 30 & 16 & 17 & 17 & 18 & 15 & 10 & 13 & 21 & 9 & 11 & 14 & 11 & 21 \\
\hline NWP68 & 16 & 13 & 21 & 31 & 19 & 16 & 16 & 18 & 14 & 10 & 11 & 21 & 11 & 11 & 13 & 11 & 21 \\
\hline NWP72 & 16 & 13 & 21 & 31 & 16 & 15 & 16 & 19 & 15 & 10 & 12 & 21 & 11 & 11 & 14 & 10 & 21 \\
\hline NWP75 & 15 & 13 & 21 & 29 & 17 & 16 & 17 & 17 & 13 & 12 & 11 & 22 & 11 & 11 & 14 & 11 & 21 \\
\hline NWP87 & 17 & 13 & 21 & 30 & 17 & 16 & 17 & 17 & 14 & 10 & 12 & 20 & 11 & 11 & 14 & 11 & 21 \\
\hline NWP93 & 16 & 13 & 21 & 30 & 17 & 17 & 15 & 17 & 15 & 10 & 11 & 21 & 11 & 11 & 14 & 11 & 21 \\
\hline NWP133 & 16 & 13 & 21 & 30 & 16 & 17 & 16 & 17 & 14 & 10 & 12 & 21 & 11 & 12 & 14 & 11 & 21 \\
\hline NWP134 & 15 & 13 & 20 & 31 & 17 & 17 & 18 & 18 & 15 & 10 & 11 & 21 & 11 & 11 & 14 & 11 & 21 \\
\hline NWP138 & 15 & 13 & 21 & 30 & 16 & 16 & 19 & 19 & 14 & 11 & 12 & 22 & 11 & 11 & 13 & 11 & 21 \\
\hline NWP181 & 16 & 13 & 21 & 30 & 16 & 16 & 16 & 18 & 13 & 10 & 11 & 21 & 11 & 11 & 14 & 11 & 22 \\
\hline NWP202 & 16 & 13 & 21 & 30 & 16 & 14 & 17 & 18 & 13 & 10 & 12 & 21 & 11 & 11 & 13 & 11 & 21 \\
\hline NWP212 & 16 & 13 & 22 & 30 & 16 & 16 & 17 & 18 & 16 & 10 & 12 & 21 & 11 & 11 & 14 & 11 & 21 \\
\hline NWP214 & 16 & 14 & 21 & 31 & 17 & 17 & 17 & 19 & 15 & 10 & 12 & 21 & 11 & 11 & 14 & 11 & 21 \\
\hline NWP216 & 16 & 13 & 21 & 30 & 16 & 17 & 16 & 18 & 14 & 10 & 11 & 22 & 11 & 11 & 14 & 11 & 21 \\
\hline NWP226 & 17 & 14 & 21 & 31 & 15 & 17 & 16 & 17 & 14 & 10 & 11 & 22 & 9 & 11 & 14 & 11 & 21 \\
\hline GRB266 & 15 & 14 & 21 & 31 & 16 & 17 & 17 & 20 & 15 & 10 & 12 & 20 & 11 & 11 & 14 & 11 & 21 \\
\hline GRB277 & 16 & 13 & 21 & 30 & 16 & 16 & 17 & 19 & 13 & 10 & 13 & 22 & 11 & 11 & 14 & 11 & 21 \\
\hline GRB306 & 16 & 12 & 21 & 29 & 16 & 17 & 17 & 20 & 15 & 10 & 13 & 21 & 11 & 11 & 13 & 11 & 21 \\
\hline GRB312 & 16 & 13 & 21 & 30 & 17 & 16 & 17 & 20 & 15 & 10 & 12 & 21 & 11 & 11 & 14 & 11 & 21 \\
\hline GRB314 & 16 & 13 & 21 & 30 & 17 & 15 & 17 & 18 & 14 & 11 & 11 & 22 & 11 & 12 & 14 & 11 & 21 \\
\hline
\end{tabular}

Supplementary Table 1: Y-STR haplotypes for E1b1a7a individuals 


\begin{tabular}{|c|c|c|c|c|c|c|c|c|c|c|c|c|c|c|c|c|c|}
\hline Sample & DYS456 & DYS389I & DYS390 & DYS389II & DYS458 & DYS19 & DYS385a & DYS385b & DYS393 & DYS391 & DYS439 & DYS635 & DYS392 & GATA_H4 & DYS437 & DYS438 & DYS448 \\
\hline GRB318 & 14 & 13 & 21 & 30 & 16 & 17 & 17 & 17 & 14 & 10 & 12 & 21 & 11 & 11 & 14 & 11 & 21 \\
\hline GRB319 & 15 & 13 & 21 & 30 & 17 & 16 & 16 & 19 & 14 & 10 & 12 & 21 & 11 & 11 & 14 & 11 & 20 \\
\hline GRB324 & 16 & 13 & 21 & 30 & 16 & 15 & 16 & 18 & 13 & 10 & 11 & 21 & 11 & 11 & 14 & 11 & 22 \\
\hline GRB327 & 16 & 13 & 21 & 30 & 16 & 17 & 16 & 17 & 14 & 10 & 11 & 21 & 11 & 11 & 14 & 11 & 21 \\
\hline GRB328 & 14 & 13 & 21 & 31 & 17 & 15 & 16 & 18 & 16 & 10 & 12 & 21 & 11 & 12 & 14 & 11 & 21 \\
\hline GRB332 & 15 & 13 & 21 & 31 & 17 & 17 & 17 & 18 & 15 & 11 & 11 & 21 & 11 & 11 & 14 & 10 & 21 \\
\hline GRB344 & 15 & 13 & 21 & 31 & 17 & 17 & 17 & 17 & 15 & 11 & 11 & 21 & 11 & 11 & 14 & 11 & 21 \\
\hline GRB345 & 15 & 13 & 21 & 30 & 16 & 17 & 17 & 20 & 13 & 9 & 12 & 23 & 11 & 11 & 14 & 11 & 21 \\
\hline ELU365 & 15 & 13 & 21 & 30 & 16 & 16 & 16 & 19 & 14 & 10 & 12 & 22 & 12 & 11 & 14 & 11 & 21 \\
\hline ELU387 & 15 & 13 & 21 & 30 & 16 & 17 & 18 & 18 & 14 & 10 & 12 & 21 & 11 & 11 & 14 & 11 & 21 \\
\hline ELU401 & 15 & 13 & 21 & 30 & 16 & 16 & 17 & 19 & 15 & 10 & 12 & 22 & 12 & 11 & 14 & 11 & 21 \\
\hline ELU408 & 17 & 13 & 21 & 30 & 17 & 16 & 17 & 17 & 14 & 10 & 12 & 20 & 11 & 11 & 14 & 11 & 21 \\
\hline ELU409 & 15 & 13 & 21 & 30 & 16 & 16 & 18 & 18 & 15 & 10 & 12 & 21 & 12 & 12 & 14 & 11 & 21 \\
\hline ELU437 & 15 & 13 & 21 & 30 & 17 & 15 & 17 & 19 & 15 & 10 & 12 & 21 & 11 & 11 & 14 & 11 & 21 \\
\hline ELU439 & 16 & 13 & 21 & 30 & 15 & 16 & 16 & 17 & 15 & 10 & 12 & 21 & 11 & 11 & 13 & 12 & 20 \\
\hline ELU446 & 16 & 14 & 21 & 31 & 17 & 17 & 17 & 20 & 15 & 10 & 13 & 21 & 11 & 11 & 14 & 11 & 21 \\
\hline ELU458 & 14 & 13 & 21 & 30 & 16 & 17 & 18 & 18 & 14 & 9 & 13 & 21 & 11 & 11 & 14 & 11 & 21 \\
\hline ELU461 & 16 & 14 & 21 & 31 & 17 & 17 & 17 & 20 & 15 & 10 & 13 & 21 & 11 & 11 & 14 & 11 & 21 \\
\hline ELU488 & 17 & 14 & 21 & 31 & 16 & 17 & 17 & 19 & 15 & 10 & 12 & 21 & 11 & 11 & 14 & 11 & 21 \\
\hline ELU760 & 16 & 13 & 21 & 31 & 17 & 15 & 16 & 18 & 15 & 10 & 12 & 21 & 11 & 11 & 14 & 11 & 21 \\
\hline LIS501 & 15 & 13 & 21 & 29 & 16 & 15 & 17 & 20 & 15 & 10 & 12 & 21 & 11 & 11 & 14 & 11 & 21 \\
\hline EXU587 & 17 & 14 & 21 & 31 & 17 & 17 & 17 & 19 & 15 & 10 & 12 & 21 & 11 & 11 & 14 & 11 & 21 \\
\hline EXU590 & 16 & 14 & 21 & 31 & 16 & 16 & 17 & 17 & 15 & 10 & 13 & 21 & 11 & 11 & 14 & 11 & 21 \\
\hline EXU592 & 16 & 13 & 21 & 30 & 16 & 15 & 16 & 17 & 15 & 10 & 12 & 21 & 11 & 11 & 13 & 11 & 21 \\
\hline EXU596 & 16 & 14 & 21 & 31 & 16 & 16 & 17 & 17 & 15 & 10 & 13 & 21 & 11 & 11 & 14 & 11 & 20 \\
\hline EXU597 & 16 & 14 & 21 & 31 & 16 & 16 & 17 & 17 & 15 & 10 & 13 & 21 & 11 & 11 & 14 & 11 & 20 \\
\hline EXU598 & 16 & 14 & 21 & 31 & 16 & 16 & 17 & 17 & 15 & 10 & 13 & 21 & 11 & 11 & 14 & 11 & 20 \\
\hline EXU605 & 16 & 13 & 21 & 31 & 16 & 17 & 17 & 18 & 14 & 10 & 12 & 21 & 10 & 11 & 14 & 11 & 21 \\
\hline
\end{tabular}

Supplementary Table 1: Y-STR haplotypes for E1b1a7a individuals (Cont'd) 


\begin{tabular}{|c|c|c|c|c|c|c|c|c|c|c|c|c|c|c|c|c|c|}
\hline Sample & DYS456 & DYS389I & DYS390 & DYS389II & DYS458 & DYS19 & DYS385a & DYS385b & DYS393 & DYS391 & DYS439 & DYS635 & DYS392 & GATA_H4 & DYS437 & DYS438 & DYS448 \\
\hline EXU630 & 16 & 13 & 21 & 30 & 16 & 16 & 16 & 18 & 15 & 11 & 11 & 21 & 11 & 11 & 14 & 11 & 21 \\
\hline EXU648 & 16 & 13 & 21 & 30 & 17 & 17 & 18 & 20 & 15 & 11 & 13 & 21 & 11 & 11 & 14 & 11 & 22 \\
\hline EXU650 & 16 & 13 & 21 & 30 & 17 & 16 & 17 & 18 & 15 & 10 & 12 & 21 & 11 & 11 & 13 & 11 & 21 \\
\hline EXU655 & 17 & 13 & 21 & 31 & 16 & 15 & 17 & 18 & 14 & 10 & 12 & 21 & 11 & 12 & 13 & 11 & 21 \\
\hline EXU656 & 15 & 13 & 21 & 30 & 16 & 17 & 18 & 19 & 14 & 10 & 12 & 21 & 11 & 11 & 14 & 11 & 21 \\
\hline EXU659 & 17 & 13 & 21 & 29 & 16 & 16 & 16 & 20 & 15 & 10 & 11 & 21 & 11 & 11 & 14 & 11 & 21 \\
\hline EXU661 & 15 & 13 & 21 & 29 & 17 & 17 & 18 & 18 & 15 & 10 & 12 & 21 & 11 & 11 & 14 & 11 & 19 \\
\hline ABA678 & 15 & 13 & 21 & 30 & 17 & 17 & 17 & 18 & 13 & 10 & 12 & 21 & 11 & 11 & 14 & 11 & 21 \\
\hline ABA695 & 16 & 13 & 21 & 30 & 17 & 16 & 17 & 20 & 15 & 10 & 12 & 21 & 11 & 11 & 14 & 11 & 21 \\
\hline ABA701 & 17 & 14 & 21 & 31 & 16 & 17 & 17 & 17 & 14 & 10 & 11 & 20 & 11 & 11 & 14 & 11 & 21 \\
\hline ABA709 & 17 & 13 & 21 & 30 & 16 & 17 & 16 & 17 & 14 & 10 & 12 & 21 & 11 & 11 & 14 & 11 & 21 \\
\hline ABA711 & 15 & 13 & 21 & 30 & 17 & 17 & 17 & 18 & 14 & 10 & 12 & 21 & 11 & 11 & 14 & 11 & 21 \\
\hline ABA718 & 16 & 13 & 21 & 30 & 17 & 17 & 16 & 18 & 15 & 10 & 12 & 21 & 11 & 11 & 14 & 11 & 21 \\
\hline ABA724 & 16 & 13 & 21 & 31 & 19 & 17 & 16 & 18 & 14 & 10 & 11 & 21 & 11 & 11 & 13 & 11 & 21 \\
\hline ABA735 & 15 & 13 & 21 & 30 & 16 & 15 & 17 & 18 & 14 & 10 & 11 & 21 & 11 & 11 & 14 & 11 & 21 \\
\hline ABA765 & 14 & 13 & 21 & 30 & 17 & 17 & 17 & 18 & 14 & 10 & 12 & 21 & 11 & 11 & 14 & 11 & 21 \\
\hline ABA768 & 16 & 14 & 21 & 31 & 16 & 17 & 18 & 20 & 15 & 10 & 12 & 21 & 11 & 11 & 13 & 11 & 21 \\
\hline ABA778 & 14 & 13 & 21 & 30 & 16 & 17 & 18 & 18 & 14 & 9 & 13 & 21 & 11 & 11 & 14 & 11 & 21 \\
\hline ABA782 & 17 & 13 & 21 & 30 & 16 & 16 & 19 & 20 & 14 & 10 & 12 & 21 & 11 & 11 & 14 & 11 & 22 \\
\hline
\end{tabular}

Supplementary Table 1: Y-STR haplotypes for E1b1a7a individuals (Cont'd) 


\begin{tabular}{|c|c|c|c|c|c|c|c|c|c|c|c|c|c|c|c|c|c|}
\hline Sample & DYS456 & DYS389I & DYS390 & DYS389II & DYS458 & DYS19 & DYS385a & DYS385b & DYS393 & DYS391 & DYS439 & DYS635 & DYS392 & GATA_H4 & DYS437 & DYS438 & DYS448 \\
\hline NWP5 & 15 & 12 & 21 & 30 & 17 & 16 & 15 & 17 & 13 & 10 & 12 & 21 & 11 & 12 & 14 & 11 & 21 \\
\hline NWP9 & 15 & 13 & 21 & 31 & 17 & 15 & 16 & 17 & 13 & 10 & 12 & 22 & 11 & 12 & 14 & 12 & 21 \\
\hline NWP12 & 15 & 14 & 21 & 32 & 17 & 15 & 16 & 18 & 13 & 11 & 12 & 22 & 11 & 12 & 14 & 11 & 21 \\
\hline NWP16 & 15 & 13 & 21 & 32 & 16 & 15 & 17 & 17 & 13 & 10 & 12 & 21 & 12 & 12 & 14 & 11 & 21 \\
\hline NWP21 & 15 & 14 & 21 & 32 & 16 & 15 & 15 & 18 & 13 & 10 & 12 & 22 & 11 & 12 & 14 & 11 & 21 \\
\hline NWP28 & 15 & 13 & 21 & 31 & 17 & 15 & 16 & 17 & 13 & 10 & 12 & 21 & 11 & 12 & 14 & 11 & 21 \\
\hline NWP36 & 16 & 14 & 21 & 33 & 15 & 17 & 17 & 19 & 14 & 10 & 12 & 21 & 11 & 12 & 14 & 11 & 21 \\
\hline NWP50 & 15 & 12 & 21 & 30 & 15 & 15 & 14 & 18 & 13 & 10 & 12 & 21 & 11 & 12 & 14 & 11 & 20 \\
\hline NWP73 & 15 & 14 & 21 & 32 & 16 & 15 & 16 & 17 & 13 & 11 & 11 & 21 & 11 & 12 & 14 & 11 & 21 \\
\hline NWP76 & 15 & 12 & 21 & 30 & 17 & 16 & 15 & 17 & 13 & 10 & 12 & 21 & 11 & 12 & 14 & 11 & 21 \\
\hline NWP84 & 15 & 14 & 21 & 31 & 18 & 15 & 15 & 16 & 14 & 10 & 12 & 21 & 11 & 12 & 14 & 11 & 21 \\
\hline NWP86 & 15 & 13 & 21 & 30 & 18 & 15 & 15 & 15 & 13 & 10 & 12 & 21 & 11 & 12 & 14 & 11 & 20 \\
\hline NWP94 & 15 & 12 & 21 & 30 & 18 & 17 & 16 & 17 & 13 & 10 & 12 & 22 & 11 & 12 & 14 & 11 & 21 \\
\hline NWP100 & 16 & 13 & 21 & 31 & 19 & 15 & 16 & 16 & 13 & 10 & 12 & 21 & 7 & 12 & 14 & 11 & 21 \\
\hline NWP112 & 13 & 14 & 21 & 32 & 15 & 15 & 16 & 18 & 13 & 10 & 13 & 21 & 11 & 11 & 14 & 11 & 21 \\
\hline NWP113 & 15 & 13 & 21 & 31 & 17 & 15 & 16 & 17 & 13 & 10 & 12 & 21 & 11 & 11 & 14 & 11 & 21 \\
\hline NWP119 & 15 & 14 & 21 & 32 & 17 & 16 & 15 & 17 & 13 & 10 & 13 & 21 & 11 & 11 & 14 & 11 & 21 \\
\hline NWP152 & 15 & 13 & 21 & 31 & 16 & 16 & 16 & 17 & 13 & 10 & 12 & 20 & 11 & 12 & 14 & 12 & 21 \\
\hline NWP156 & 15 & 13 & 21 & 31 & 17 & 16 & 16 & 17 & 13 & 10 & 11 & 21 & 11 & 12 & 14 & 11 & 20 \\
\hline NWP160 & 14 & 14 & 21 & 33 & 17 & 16 & 16 & 16 & 13 & 10 & 12 & 21 & 11 & 11 & 14 & 11 & 21 \\
\hline NWP178 & 15 & 14 & 21 & 31 & 18 & 16 & 16 & 18 & 13 & 10 & 12 & 21 & 11 & 12 & 13 & 11 & 21 \\
\hline NWP188 & 14 & 13 & 21 & 31 & 17 & 15 & 16 & 17 & 13 & 10 & 12 & 21 & 11 & 12 & 14 & 12 & 21 \\
\hline NWP190 & 15 & 13 & 21 & 31 & 17 & 16 & 16 & 17 & 13 & 10 & 11 & 21 & 11 & 12 & 14 & 11 & 20 \\
\hline NWP206 & 15 & 13 & 21 & 30 & 18 & 15 & 15 & 15 & 13 & 10 & 12 & 21 & 11 & 12 & 14 & 11 & 20 \\
\hline NWP208 & 15 & 13 & 21 & 32 & 15 & 16 & 15 & 16 & 13 & 10 & 12 & 23 & 11 & 12 & 14 & 11 & 21 \\
\hline NWP213 & 14 & 12 & 21 & 30 & 17 & 15 & 15 & 19 & 13 & 10 & 12 & 21 & 11 & 12 & 14 & 11 & 21 \\
\hline NWP215 & 15 & 13 & 21 & 31 & 16 & 15 & 16 & 17 & 13 & 11 & 11 & 21 & 11 & 12 & 14 & 11 & 21 \\
\hline NWP217 & 14 & 13 & 21 & 31 & 16 & 15 & 16 & 17 & 13 & 11 & 11 & 21 & 11 & 12 & 14 & 11 & 21 \\
\hline GRB227 & 15 & 13 & 21 & 30 & 17 & 15 & 16 & 17 & 13 & 11 & 13 & 21 & 11 & 12 & 14 & 11 & 21 \\
\hline GRB228 & 15 & 13 & 21 & 30 & 19 & 15 & 14 & 18 & 14 & 10 & 12 & 21 & 11 & 11 & 14 & 11 & 21 \\
\hline
\end{tabular}

Supplementary Table 2: Y-STR haplotypes for E1b1a8 individuals 


\begin{tabular}{|c|c|c|c|c|c|c|c|c|c|c|c|c|c|c|c|c|c|}
\hline Sample & DYS456 & DYS389I & DYS390 & DYS389II & DYS458 & DYS19 & DYS385a & DYS385b & DYS393 & DYS391 & DYS439 & DYS635 & DYS392 & GATA_H4 & DYS437 & DYS438 & DYS448 \\
\hline GRB244 & 16 & 13 & 21 & 31 & 18 & 15 & 16 & 18 & 13 & 10 & 12 & 21 & 11 & 12 & 15 & 11 & 21 \\
\hline GRB271 & 15 & 14 & 21 & 31 & 18 & 15 & 15 & 16 & 14 & 10 & 12 & 21 & 11 & 12 & 14 & 11 & 21 \\
\hline GRB275 & 16 & 13 & 21 & 32 & 17 & 16 & 16 & 17 & 14 & 10 & 11 & 21 & 12 & 12 & 14 & 11 & 20 \\
\hline GRB322 & 15 & 13 & 21 & 31 & 16 & 17 & 16 & 17 & 13 & 10 & 12 & 21 & 11 & 12 & 14 & 11 & 21 \\
\hline GRB326 & 15 & 12 & 21 & 32 & 15 & 16 & 16 & 18 & 13 & 9 & 13 & 21 & 11 & 12 & 14 & 11 & 21 \\
\hline GRB334 & 15 & 13 & 21 & 31 & 17 & 15 & 15 & 16 & 13 & 10 & 12 & 21 & 11 & 11 & 14 & 12 & 21 \\
\hline GRB341 & 15 & 13 & 21 & 31 & 16 & 15 & 16 & 17 & 13 & 11 & 11 & 21 & 11 & 12 & 14 & 11 & 21 \\
\hline GRB343 & 15 & 12 & 21 & 29 & 17 & 15 & 16 & 18 & 13 & 10 & 12 & 22 & 11 & 12 & 14 & 11 & 21 \\
\hline GRB355 & 15 & 13 & 21 & 31 & 16 & 15 & 15 & 17 & 13 & 10 & 11 & 22 & 12 & 12 & 14 & 11 & 21 \\
\hline ELU376 & 16 & 13 & 21 & 32 & 19 & 16 & 17 & 17 & 14 & 10 & 11 & 21 & 11 & 12 & 14 & 11 & 21 \\
\hline ELU378 & 16 & 13 & 21 & 30 & 17 & 15 & 15 & 17 & 13 & 11 & 12 & 21 & 11 & 12 & 14 & 11 & 21 \\
\hline ELU380 & 14 & 13 & 21 & 31 & 16 & 15 & 16 & 16 & 13 & 10 & 12 & 21 & 11 & 12 & 14 & 11 & 21 \\
\hline ELU395 & 16 & 13 & 21 & 30 & 18 & 16 & 17 & 18 & 13 & 10 & 12 & 21 & 11 & 12 & 14 & 11 & 22 \\
\hline ELU397 & 16 & 13 & 21 & 30 & 17 & 15 & 15 & 17 & 13 & 11 & 12 & 21 & 11 & 12 & 14 & 11 & 21 \\
\hline ELU416 & 15 & 13 & 21 & 30 & 16 & 15 & 16 & 18 & 13 & 10 & 12 & 22 & 11 & 12 & 14 & 11 & 21 \\
\hline ELU421 & 15 & 13 & 21 & 31 & 15 & 15 & 16 & 18 & 13 & 10 & 12 & 21 & 11 & 12 & 14 & 11 & 22 \\
\hline ELU425 & 14 & 13 & 21 & 30 & 17 & 15 & 14 & 15 & 13 & 10 & 12 & 23 & 11 & 11 & 14 & 11 & 21 \\
\hline ELU426 & 15 & 13 & 21 & 31 & 17 & 16 & 16 & 17 & 13 & 10 & 11 & 21 & 11 & 12 & 14 & 11 & 20 \\
\hline ELU440 & 15 & 12 & 21 & 30 & 17 & 16 & 16 & 18 & 13 & 10 & 12 & 21 & 11 & 12 & 14 & 11 & 21 \\
\hline ELU454 & 15 & 13 & 21 & 31 & 16 & 15 & 16 & 18 & 13 & 11 & 12 & 21 & 11 & 12 & 14 & 11 & 21 \\
\hline LIS471 & 16 & 13 & 21 & 31 & 17 & 15 & 17 & 17 & 13 & 10 & 12 & 21 & 11 & 12 & 14 & 11 & 21 \\
\hline LIS472 & 15 & 13 & 21 & 31 & 16 & 15 & 16 & 17 & 13 & 10 & 11 & 22 & 11 & 12 & 14 & 12 & 21 \\
\hline LIS498 & 15 & 13 & 22 & 32 & 17 & 15 & 15 & 16 & 13 & 10 & 13 & 22 & 11 & 12 & 13 & 11 & 21 \\
\hline LIS516 & 14 & 13 & 21 & 30 & 17 & 15 & 14 & 15 & 13 & 10 & 12 & 23 & 11 & 11 & 14 & 11 & 21 \\
\hline LIS536 & 15 & 13 & 21 & 31 & 16 & 17 & 16 & 17 & 13 & 10 & 12 & 21 & 11 & 12 & 14 & 11 & 21 \\
\hline LIS547 & 15 & 13 & 21 & 30 & 17 & 15 & 17 & 18 & 13 & 10 & 11 & 21 & 11 & 12 & 14 & 11 & 21 \\
\hline EXU572 & 15 & 13 & 21 & 31 & 17 & 15 & 16 & 17 & 13 & 10 & 11 & 21 & 11 & 12 & 14 & 12 & 21 \\
\hline EXU573 & 15 & 12 & 21 & 32 & 15 & 16 & 16 & 18 & 13 & 9 & 13 & 21 & 11 & 12 & 14 & 11 & 21 \\
\hline EXU582 & 16 & 13 & 21 & 30 & 20 & 15 & 16 & 17 & 13 & 10 & 12 & 21 & 11 & 13 & 14 & 11 & 21 \\
\hline EXU586 & 15 & 12 & 21 & 32 & 15 & 16 & 16 & 18 & 13 & 9 & 14 & 21 & 11 & 12 & 14 & 11 & 21 \\
\hline
\end{tabular}

Supplementary Table 2: Y-STR haplotypes for E1b1a8 individuals (Cont'd) 


\begin{tabular}{|c|c|c|c|c|c|c|c|c|c|c|c|c|c|c|c|c|c|}
\hline Sample & DYS456 & DYS389I & DYS390 & DYS389II & DYS458 & DYS19 & DYS385a & DYS385b & DYS393 & DYS391 & DYS439 & DYS635 & DYS392 & GATA_H4 & DYS437 & DYS438 & DYS448 \\
\hline EXU591 & 16 & 13 & 21 & 31 & 20 & 15 & 16 & 17 & 13 & 10 & 12 & 21 & 11 & 13 & 14 & 11 & 21 \\
\hline EXU594 & 15 & 12 & 21 & 31 & 15 & 16 & 16 & 18 & 13 & 9 & 13 & 21 & 11 & 12 & 14 & 11 & 21 \\
\hline EXU595 & 15 & 13 & 21 & 31 & 18 & 15 & 14 & 15 & 13 & 10 & 12 & 21 & 11 & 14 & 14 & 11 & 21 \\
\hline EXU623 & 15 & 13 & 21 & 32 & 16 & 15 & 15 & 16 & 13 & 10 & 12 & 21 & 11 & 12 & 14 & 11 & 21 \\
\hline EXU632 & 15 & 14 & 21 & 32 & 17 & 13 & 17 & 20 & 13 & 10 & 12 & 21 & 11 & 12 & 14 & 11 & 22 \\
\hline EXU641 & 15 & 13 & 21 & 31 & 16 & 15 & 16 & 17 & 13 & 10 & 12 & 22 & 11 & 12 & 14 & 12 & 21 \\
\hline EXU645 & 15 & 13 & 21 & 31 & 16 & 15 & 17 & 18 & 13 & 10 & 12 & 21 & 11 & 12 & 14 & 11 & 21 \\
\hline ABA671 & 15 & 14 & 21 & 33 & 17 & 13 & 17 & 21 & 13 & 10 & 12 & 21 & 11 & 12 & 15 & 11 & 22 \\
\hline ABA672 & 15 & 12 & 21 & 32 & 15 & 16 & 16 & 18 & 13 & 9 & 14 & 21 & 11 & 12 & 14 & 11 & 21 \\
\hline ABA674 & 15 & 13 & 21 & 30 & 16 & 16 & 15 & 18 & 14 & 10 & 13 & 21 & 10 & 12 & 14 & 11 & 21 \\
\hline ABA676 & 15 & 13 & 21 & 30 & 16 & 16 & 15 & 18 & 14 & 10 & 13 & 21 & 10 & 12 & 14 & 11 & 21 \\
\hline ABA677 & 15 & 13 & 21 & 30 & 16 & 15 & 16 & 17 & 13 & 11 & 11 & 21 & 11 & 12 & 14 & 11 & 20 \\
\hline $\mathrm{ABA} 680$ & 15 & 12 & 21 & 30 & 15 & 15 & 14 & 18 & 13 & 10 & 12 & 21 & 11 & 12 & 14 & 11 & 21 \\
\hline ABA685 & 15 & 13 & 21 & 30 & 16 & 16 & 15 & 18 & 14 & 10 & 13 & 21 & 10 & 12 & 14 & 11 & 21 \\
\hline ABA696 & 15 & 13 & 20 & 30 & 17 & 16 & 14 & 20 & 14 & 10 & 13 & 22 & 11 & 12 & 14 & 11 & 21 \\
\hline ABA698 & 15 & 13 & 20 & 30 & 17 & 16 & 14 & 20 & 14 & 10 & 13 & 22 & 11 & 12 & 14 & 11 & 21 \\
\hline ABA702 & 15 & 12 & 21 & 32 & 15 & 16 & 15 & 18 & 13 & 9 & 14 & 21 & 11 & 12 & 14 & 11 & 21 \\
\hline $\mathrm{ABA} 703$ & 14 & 13 & 21 & 31 & 16 & 15 & 14 & 16 & 13 & 10 & 12 & 22 & 13 & 11 & 14 & 11 & 22 \\
\hline ABA706 & 15 & 13 & 21 & 32 & 17 & 15 & 15 & 17 & 13 & 10 & 12 & 21 & 11 & 11 & 14 & 11 & 21 \\
\hline ABA727 & 15 & 13 & 21 & 31 & 18 & 15 & 15 & 17 & 13 & 10 & 13 & 21 & 11 & 11 & 14 & 11 & 21 \\
\hline ABA728 & 15 & 13 & 21 & 31 & 17 & 15 & 16 & 17 & 13 & 10 & 12 & 21 & 11 & 12 & 14 & 11 & 21 \\
\hline ABA742 & 15 & 12 & 21 & 29 & 17 & 15 & 15 & 15 & 13 & 10 & 13 & 21 & 11 & 13 & 14 & 12 & 21 \\
\hline ABA747 & 16 & 14 & 21 & 31 & 18 & 16 & 16 & 17 & 14 & 10 & 12 & 22 & 11 & 11 & 14 & 11 & 21 \\
\hline ABA762 & 15 & 13 & 21 & 32 & 17 & 15 & 17 & 17 & 13 & 10 & 13 & 21 & 11 & 12 & 14 & 11 & 21 \\
\hline
\end{tabular}

Supplementary Table 2: Y-STR haplotypes for E1b1a8 individuals (Cont'd) 


\begin{tabular}{|c|c|c|c|c|c|c|c|c|c|c|c|c|c|c|c|c|c|}
\hline Sample & DYS456 & DYS389I & DYS390 & DYS389II & DYS458 & DYS19 & DYS385a & DYS385b & DYS393 & DYS391 & DYS439 & DYS635 & DYS392 & GATA_H4 & DYS437 & DYS438 & DYS448 \\
\hline NWP 2 & 16 & 13 & 24 & 29 & 16 & 14 & 11 & 14 & 13 & 11 & 13 & 23 & 13 & 12 & 15 & 11 & 19 \\
\hline NWP 13 & 16 & 14 & 24 & 30 & 17 & 14 & 10 & 14 & 13 & 11 & 12 & 24 & 13 & 13 & 14 & 12 & 19 \\
\hline NWP 15 & 15 & 13 & 25 & 29 & 17 & 14 & 11 & 11 & 13 & 10 & 12 & 23 & 13 & 11 & 14 & 11 & 18 \\
\hline NWP 20 & 18 & 14 & 23 & 30 & 18 & 15 & 11 & 13 & 13 & 11 & 11 & 23 & 13 & 11 & 15 & 12 & 19 \\
\hline NWP 37 & 16 & 13 & 25 & 29 & 17 & 14 & 11 & 13 & 13 & 11 & 13 & 23 & 14 & 12 & 15 & 12 & 18 \\
\hline NWP 40 & 16 & 13 & 24 & 30 & 16 & 14 & 11 & 14 & 13 & 11 & 13 & 23 & 13 & 12 & 15 & 11 & 19 \\
\hline NWP 43 & 16 & 13 & 24 & 29 & 16 & 14 & 11 & 14 & 13 & 11 & 12 & 23 & 13 & 12 & 15 & 12 & 19 \\
\hline NWP 44 & 15 & 13 & 24 & 30 & 17 & 13 & 11 & 14 & 14 & 10 & 12 & 23 & 13 & 14 & 15 & 12 & 18 \\
\hline NWP 54 & 15 & 12 & 24 & 29 & 17,18 & 14 & 11 & 15 & 13 & 11 & 12 & 23 & 13 & 12 & 14 & 12 & 19 \\
\hline NWP 56 & 15 & 13 & 24 & 29 & 16 & 14 & 11 & 14 & 13 & 11 & 13 & 23 & 13 & 11 & 14 & 12 & 18 \\
\hline NWP 60 & 16 & 13 & 24 & 30 & 16 & 14 & 11 & 14 & 12 & 11 & 12 & 23 & 13 & 11 & 15 & 12 & 19 \\
\hline NWP 63 & 16 & 13 & 24 & 29 & 17 & 15 & 11 & 14 & 13 & 11 & 12 & 23 & 13 & 12 & 15 & 12 & 19 \\
\hline NWP 98 & 15 & 13 & 25 & 29 & 17 & 14 & 12 & 12 & 13 & 11 & 12 & 23 & 13 & 13 & 15 & 12 & 19 \\
\hline NWP 102 & 16 & 13 & 25 & 29 & 17 & 14 & 11 & 14 & 13 & 10 & 11 & 23 & 13 & 12 & 15 & 12 & 18 \\
\hline NWP 106 & 17 & 13 & 23 & 29 & 17 & 14 & 11 & 14 & 13 & 11 & 12 & 23 & 13 & 12 & 15 & 12 & 19 \\
\hline NWP 125 & 15 & 13 & 25 & 29 & 17 & 14 & 11 & 14 & 13 & 11 & 12 & 23 & 13 & 12 & 15 & 12 & 19 \\
\hline NWP 176 & 16 & 13 & 24 & 30 & 17 & 12 & 11 & 15 & 13 & 10 & 13 & 24 & 13 & 12 & 15 & 12 & 19 \\
\hline NWP 179 & 16 & 13 & 24 & 29 & 21 & 14 & 11 & 14 & 13 & 11 & 12 & 23 & 13 & 12 & 15 & 12 & 19 \\
\hline NWP 205 & 15 & 13 & 25 & 29 & 16 & 14 & 11 & 14 & 13 & 11 & 12 & 23 & 13 & 12 & 15 & 12 & 19 \\
\hline NWP 209 & 16 & 13 & 24 & 29 & 18 & 14 & 11 & 14 & 13 & 10 & 12 & 23 & 13 & 12 & 15 & 12 & 19 \\
\hline NWP 220 & 14 & 13 & 24 & 29 & 20 & 14 & 11 & 14 & 13 & 11 & 12 & 23 & 14 & 12 & 15 & 12 & 19 \\
\hline NWP 221 & 16 & 13 & 24 & 29 & 17 & 14 & 11 & 14 & 13 & 11 & 12 & 23 & 13 & 12 & 15 & 11 & 19 \\
\hline NWP 223 & 16 & 13 & 24 & 29 & 17 & 14 & 11 & 11 & 13 & 11 & 12 & 23 & 13 & 11 & 14 & 12 & 18 \\
\hline NWP 784 & 16 & 13 & 23 & 29 & 17 & 14 & 11 & 15 & 13 & 11 & 11 & 23 & 15 & 12 & 15 & 12 & 19 \\
\hline GRB 232 & 16 & 13 & 23 & 28 & 17 & 14 & 11 & 11 & 13 & 11 & 11 & 23 & 13 & 12 & 15 & 12 & 18 \\
\hline GRB 233 & 16 & 13 & 24 & 29 & 16 & 14 & 11 & 13 & 13 & 11 & 11 & 23 & 13 & 12 & 15 & 12 & 19 \\
\hline GRB 246 & 16 & 14 & 23 & 30 & 17 & 14 & 11 & 14 & 13 & 10 & 12 & 23 & 13 & 12 & 15 & 12 & 19 \\
\hline GRB 315 & 16 & 13 & 24 & 29 & 17 & 14 & 11 & 14 & 13 & 11 & 12 & 23 & 13 & 12 & 15 & 12 & 19 \\
\hline
\end{tabular}

\section{Supplementary Table 3: Y-STR haplotypes for R1b1b1 individuals}




\begin{tabular}{|c|c|c|c|c|c|c|c|c|c|c|c|c|c|c|c|c|c|}
\hline Sample & DYS456 & DYS389I & DYS390 & DYS389II & DYS458 & DYS19 & DYS385a & DYS385b & DYS393 & DYS391 & DYS439 & DYS635 & DYS392 & GATA_H4 & DYS437 & DYS438 & DYS448 \\
\hline GRB 321 & 15 & 13 & 24 & 29 & 17 & 14 & 11 & 14 & 13 & 10 & 12 & 23 & 13 & 12 & 15 & 12 & 19 \\
\hline GRB 329 & 16 & 13 & 25 & 29 & 16 & 14 & 11 & 14 & 13 & 11 & 11 & 23 & 13 & 12 & 15 & 12 & 19 \\
\hline GRB 339 & 15 & 13 & 24 & 29 & 17 & 14 & 11 & 14 & 13 & 11 & 13 & 23 & 13 & 12 & 15 & 12 & 19 \\
\hline GRB 354 & 15 & 13 & 24 & 29 & 18 & 14 & 11 & 13 & 13 & 12 & 12 & 23 & 13 & 12 & 15 & 12 & 19 \\
\hline ELU 364 & 16 & 13 & 24 & 29 & 17 & 14 & 11 & 14 & 14 & 10 & 12 & 23 & 13 & 12 & 15 & 12 & 19 \\
\hline ELU 403 & 15 & 14 & 23 & 30 & 17 & 14 & 11 & 14 & 13 & 11 & 12 & 23 & 13 & 11 & 15 & 12 & 19 \\
\hline ELU 404 & 16 & 13 & 25 & 29 & 17 & 14 & 12 & 14 & 13 & 11 & 13 & 23 & 13 & 12 & 15 & 12 & 19 \\
\hline ELU 430 & 15 & 13 & 24 & 29 & 17 & 14 & 11 & 15 & 13 & 10 & 11 & 24 & 13 & 12 & 15 & 12 & 19 \\
\hline ELU 467 & 16 & 13 & 25 & 30 & 17 & 14 & 11 & 15 & 13 & 11 & 12 & 23 & 12 & 12 & 15 & 12 & 18 \\
\hline LIS 469 & 15 & 13 & 23 & 28 & 16 & 14 & 11 & 14 & 13 & 10 & 11 & 23 & 13 & 12 & 15 & 12 & 19 \\
\hline LIS 473 & 15 & 13 & 24 & 30 & 17 & 13 & 11 & 14 & 14 & 10 & 12 & 23 & 13 & 13 & 15 & 12 & 18 \\
\hline LIS 474 & 15 & 13 & 24 & 30 & 17 & 13 & 11 & 14 & 14 & 10 & 12 & 23 & 13 & 13 & 15 & 12 & 18 \\
\hline LIS 475 & 16 & 13 & 25 & 29 & 17 & 14 & 11 & 14 & 13 & 10 & 11 & 23 & 13 & 12 & 15 & 12 & 18 \\
\hline LIS 476 & 15 & 14 & 23 & 30 & 16 & 14 & 11 & 14 & 13 & 10 & 14 & 23 & 13 & 12 & 15 & 12 & 19 \\
\hline LIS 478 & 16 & 12 & 24 & 28 & 18 & 15 & 11 & 15 & 13 & 11 & 12 & 23 & 13 & 13 & 15 & 12 & 19 \\
\hline LIS 483 & 17 & 13 & 23 & 29 & 17 & 14 & 11 & 14 & 13 & 11 & 12 & 23 & 13 & 12 & 15 & 12 & 19 \\
\hline LIS 489 & 15 & 13 & 23 & 28 & 16 & 14 & 11 & 14 & 13 & 10 & 11 & 23 & 13 & 12 & 15 & 12 & 19 \\
\hline LIS 499 & 16 & 12 & 24 & 28 & 18 & 15 & 11 & 15 & 13 & 11 & 12 & 23 & 13 & 13 & 15 & 12 & 19 \\
\hline LIS 500 & 16 & 13 & 24 & 28 & 17 & 15 & 11 & 15 & 13 & 11 & 13 & 23 & 13 & 12 & 15 & 12 & 19 \\
\hline LIS 506 & 15 & 13 & 24 & 29 & 18 & 14 & 11 & 14 & 13 & 10 & 11 & 23 & 15 & 12 & 15 & 12 & 19 \\
\hline LIS 517 & 15 & 13 & 24 & 29 & 18 & 14 & 11 & 14 & 13 & 10 & 11 & 23 & 15 & 12 & 15 & 12 & 19 \\
\hline LIS 518 & 16 & 13 & 25 & 29 & 18 & 14 & 11 & 14 & 13 & 10 & 11 & 23 & 13 & 12 & 15 & 12 & 18 \\
\hline LIS 519 & 15 & 13 & 24 & 29 & 15 & 14 & 11 & 14 & 12 & 10 & 12 & 24 & 13 & 12 & 15 & 12 & 19 \\
\hline LIS 520 & 15 & 13 & 24 & 29 & 18 & 14 & 11 & 14 & 13 & 10 & 11 & 23 & 15 & 12 & 15 & 12 & 19 \\
\hline LIS 522 & 16 & 13 & 24 & 28 & 17 & 15 & 11 & 15 & 13 & 11 & 13 & 23 & 13 & 12 & 15 & 12 & 19 \\
\hline LIS 531 & 17 & 12 & 25 & 28 & 17 & 14 & 11 & 13 & 13 & 11 & 12 & 23 & 14 & 12 & 15 & 12 & 18 \\
\hline LIS 532 & 15 & 13 & 24 & 29 & 18 & 14 & 11 & 14 & 13 & 11 & 12 & 24 & 13 & 12 & 15 & 12 & 19 \\
\hline LIS 541 & 15 & 13 & 24 & 29 & 15 & 14 & 11 & 14 & 12 & 10 & 12 & 24 & 13 & 12 & 15 & 12 & 19 \\
\hline
\end{tabular}

Supplementary Table 3: Y-STR haplotypes for R1b1b1 individuals (Cont'd) 


\begin{tabular}{|c|c|c|c|c|c|c|c|c|c|c|c|c|c|c|c|c|c|}
\hline Sample & DYS456 & DYS389I & DYS390 & DYS389II & DYS458 & DYS19 & DYS385a & DYS385b & DYS393 & DYS391 & DYS439 & DYS635 & DYS392 & GATA_H4 & DYS437 & DYS438 & DYS448 \\
\hline LIS 542 & 15 & 13 & 24 & 29 & 16 & 14 & 11 & 14 & 13 & 11 & 13 & 23 & 13 & 11 & 14 & 12 & 18 \\
\hline LIS 545 & 16 & 13 & 24 & 29 & 17 & 14 & 10 & 14 & 13 & 11 & 12 & 24 & 13 & 13 & 14 & 12 & 19 \\
\hline LIS 551 & 15 & 13 & 24 & 29 & 16 & 14 & 11 & 14 & 13 & 11 & 13 & 23 & 13 & 11 & 14 & 12 & 18 \\
\hline EXU 610 & 16 & 12 & 24 & 27 & 16 & 14 & 11 & 15 & 13 & 11 & 11 & 23 & 13 & 12 & 15 & 12 & 19 \\
\hline EXU 622 & 16 & 13 & 23 & 29 & 16 & 14 & 11 & 15 & 13 & 11 & 12 & 25 & 13 & 12 & 14 & 12 & 19 \\
\hline EXU 626 & 17 & 13 & 23 & 29 & 17 & 14 & 11 & 14 & 13 & 11 & 12 & 23 & 13 & 12 & 15 & 12 & 19 \\
\hline EXU 647 & 16 & 13 & 23 & 28 & 16 & 14 & 12 & 14 & 13 & 10 & 11 & 23 & 13 & 12 & 15 & 12 & 19 \\
\hline EXU 662 & 17 & 13 & 23 & 29 & 17 & 14 & 11 & 14 & 13 & 11 & 12 & 23 & 13 & 12 & 15 & 12 & 19 \\
\hline ABA 669 & 15 & 12 & 23 & 28 & 18 & 14 & 11 & 14 & 13 & 11 & 12 & 23 & 13 & 12 & 15 & 12 & 19 \\
\hline ABA 679 & 16 & 14 & 23 & 30 & 17 & 14 & 11 & 14 & 13 & 11 & 12 & 25 & 13 & 12 & 15 & 12 & 18 \\
\hline ABA 681 & 15 & 12 & 24 & 28 & 17 & 14 & 12 & 14 & 13 & 11 & 13 & 23 & 13 & 12 & 16 & 12 & 19 \\
\hline ABA 699 & 15 & 13 & 21 & 29 & 18 & 14 & 11 & 15 & 13 & 11 & 11 & 24 & 13 & 12 & 15 & 12 & 19 \\
\hline ABA 700 & 16 & 12 & 23 & 28 & 18 & 14 & 11 & 14 & 13 & 11 & 12 & 23 & 13 & 12 & 15 & 12 & 19 \\
\hline ABA 731 & 16 & 13 & 25 & 30 & 17 & 14 & 11 & 15 & 13 & 11 & 12 & 23 & 12 & 12 & 15 & 12 & 18 \\
\hline ABA 732 & 17 & 14 & 23 & 30 & 20 & 14 & 11 & 14 & 13 & 10 & 11 & 24 & 13 & 11 & 15 & 12 & 19 \\
\hline ABA 744 & 16 & 13 & 25 & 30 & 17 & 14 & 11 & 15 & 13 & 12 & 12 & 23 & 12 & 12 & 15 & 12 & 18 \\
\hline ABA 766 & 16 & 13 & 23 & 30 & 16 & 14 & 11 & 12 & 13 & 11 & 14 & 23 & 13 & 12 & 15 & 12 & 19 \\
\hline ABA 767 & 16 & 13 & 24 & 29 & 16 & 14 & 11 & 14 & 13 & 11 & 13 & 23 & 13 & 12 & 15 & 11 & 19 \\
\hline
\end{tabular}

Supplementary Table 3: Y-STR haplotypes for R1b1b1 individuals (Cont'd) 


\begin{tabular}{|c|c|c|c|}
\hline Haplogroup and Population & Abbreviation & $\mathbf{N}$ & References \\
\hline \multicolumn{4}{|l|}{ E1b1a7a } \\
\hline Abaco & ABA & 13 & present study \\
\hline Angola & ANG & 46 & Coelho et al., 2009; de Filippo et al., 2011 \\
\hline Benin (Beninese) & BEN & 20 & Brucato et al., 2010 \\
\hline Botswana & BOT & 10 & de Filippo et al., 2011 \\
\hline Burkina Faso (Gur \& Mande) & BRF & 13 & de Filippo et al., 2011 \\
\hline Cameroon (Bantu) & $\mathrm{CAB}$ & 13 & Berniell-Lee et al., 2009; de Filippo et al., 2011 \\
\hline Cameroon (Pygmy) & CPY & 10 & Berniell-Lee et al., 2009; de Filippo et al., 2011 \\
\hline Central African Republic (Pygmy) & CRP & 10 & de Filippo et al., 2011 \\
\hline Democratic Republic of the Congo (Bantu) & DRC & 16 & de Filippo et al., 2011 \\
\hline Democratic Republic of the Congo (Pygmy) & DRP & 3 & de Filippo et al., 2011 \\
\hline Eleuthera & ELU & 13 & present study \\
\hline Exuma & EXU & 14 & present study \\
\hline Gabon (Bantu) & GAB & 302 & Berniell-Lee et al., 2009; de Filippo et al., 2011 \\
\hline Gabon (Pygmy) & GAP & 3 & Berniell-Lee et al., 2009; de Filippo et al., 2011 \\
\hline Grand Bahama & GRB & 14 & present study \\
\hline Ivory Coast (Ahizi \& Yacouba) & IVC & 13 & Brucato et al., 2010 \\
\hline Kenya (Bantu) & KEB & 4 & de Filippo et al., 2011 \\
\hline Kenya (Nilotic) & KEN & 10 & de Filippo et al., 2011 \\
\hline Long Island & LIS & 1 & present study \\
\hline New Providence & NWP & 25 & present study \\
\hline Nigeria & NIG & 8 & de Filippo et al., 2011 \\
\hline Tanzania (Bantu) & $\mathrm{TAB}$ & 15 & Tishkoff et al., 2007; de Filippo et al., 2011 \\
\hline Tanzania (Khoisan) & TAK & 19 & Tishkoff et al., 2007; de Filippo et al., 2011 \\
\hline Uganda (Nilotes) & UGA & 6 & Gomes et al., 2010; de Filippo et al., 2011 \\
\hline Zambia (East \& West Bantu) & ZAM & 125 & de Filippo et al., 2011 \\
\hline
\end{tabular}

Supplementary Table 4: Populations examined in E1b1a7a-U174 Y-STR based analyses 


\begin{tabular}{|c|c|c|c|}
\hline Haplogroup and Population & Abbreviation & $\mathbf{N}$ & References \\
\hline \multicolumn{4}{|l|}{ E1b1a8 } \\
\hline Abaco & $\mathrm{ABA}$ & 17 & present study \\
\hline Angola & ANG & 143 & Coelho et al., 2009; de Filippo et al., 2011 \\
\hline Benin (Beninese) & BEN & 7 & Brucato et al., 2010 \\
\hline Botswana & BOT & 13 & Filippo et al., 2010 \\
\hline Burkina Faso (Gur \& Mande) & $\mathrm{BRF}$ & 86 & Filippo et al., 2010 \\
\hline Cameroon (Bantu) & $\mathrm{CAB}$ & 6 & Berniell-Lee et al., 2009; de Filippo et al., 2011 \\
\hline Cameroon (Pygmy) & CAP & 3 & Berniell-Lee et al., 2009; de Filippo et al., 2011 \\
\hline Central African Republic (Pygmy) & CRP & 6 & de Filippo et al., 2011 \\
\hline Democratic Republic of the Congo (Bantu) & $\mathrm{DRC}$ & 22 & de Filippo et al., 2011 \\
\hline Democratic Republic of the Congo (Pygmy) & DRP & 1 & de Filippo et al., 2011 \\
\hline Eleuthera & ELU & 11 & present study \\
\hline Exuma & EXU & 11 & present study \\
\hline Gabon (Bantu) & GAB & 289 & Berniell-Lee et al., 2009; de Filippo et al., 2011 \\
\hline Gabon (Pygmy) & GAP & 1 & Berniell-Lee et al., 2009; de Filippo et al., 2011 \\
\hline Grand Bahama & GRB & 11 & present study \\
\hline Ivory Coast (Ahizi \& Yacouba) & IVC & 31 & Brucato et al., 2010 \\
\hline Kenya (Bantu) & KEB & 2 & de Filippo et al., 2011 \\
\hline Kenya (Nilotic) & KEN & 6 & de Filippo et al., 2011 \\
\hline Nigeria & NIG & 1 & de Filippo et al., 2011 \\
\hline Long Island & LIS & 6 & present study \\
\hline New Providence & NWP & 28 & present study \\
\hline Senegal & SEN & 1 & de Filippo et al., 2011 \\
\hline Tanzania (Bantu) & TAB & 13 & Tishkoff et al., 2007; de Filippo et al., 2011 \\
\hline Tanzania (Khoisan) & TAK & 22 & Tishkoff et al., 2007; de Filippo et al., 2011 \\
\hline Uganda (Nilotes) & UGA & 7 & Gomes et al., 2010; de Filippo et al., 2011 \\
\hline Zambia (East \& West Bantu) & ZAM & 309 & de Filippo et al., 2011 \\
\hline
\end{tabular}

\section{Supplementary Table 5: Populations examined in E1b1a8-U175 Y-STR based analyses}




\begin{tabular}{|c|c|c|c|}
\hline Haplogroup and Population & Abbreviation & $\mathbf{N}$ & References \\
\hline \multicolumn{4}{|l|}{ R1b1b1 } \\
\hline Abaco & $\mathrm{ABA}$ & 11 & present study \\
\hline Eleuthera & ELU & 6 & present study \\
\hline Exuma & EXU & 5 & present study \\
\hline Greece (North) & GRE & 19 & Kovatsi, Saunier and Irwin, 2009 \\
\hline Grand Bahama & GRB & 8 & present study \\
\hline Ireland & IRE & 124 & Ballard et al., 2006 \\
\hline Italy & ITA & 57 & Onofri et al., 2007 \\
\hline Long Island & LIS & 24 & present study \\
\hline Macedonia & MAC & 20 & Spiroski et al., 2005 \\
\hline Montenegro & MON & 38 & Mirabal et al., 2010 \\
\hline New Providence & NWP & 26 & present study \\
\hline Poland & POL & 18 & Rębała and Szczerkowska, 2005 \\
\hline Portugal & POR & 136 & Alves et al., 2007 \\
\hline Romania & ROM & 15 & Stanciu et al., 2010 \\
\hline Serbia & SER & 8 & Mirabal et al., 2010 \\
\hline Spain & SPN & 88 & Martin et al., 2004 \\
\hline United Kindgom (Caucasian) & UKC & 165 & Ballard et al., 2005 \\
\hline
\end{tabular}

\section{Supplementary Table 6: Populations examined in R1b1b1-M269 Y-STR based analyses}




\begin{tabular}{|c|c|c|c|c|c|c|c|}
\hline & Population & Abbreviation & $\mathbf{N}$ & $\begin{array}{c}\text { Number of different } \\
\text { haplotypes }\end{array}$ & $\begin{array}{c}\text { Number of unique } \\
\text { haplotypes }\end{array}$ & $\begin{array}{c}\text { Haplotype } \\
\text { diversity (HD) }\end{array}$ & $\begin{array}{c}\text { Mean number of } \\
\text { pairwise differences }\end{array}$ \\
\hline \multirow{6}{*}{$\sum_{\substack{\infty \\
\infty}}^{\sum_{\infty}^{\infty}}$} & Abaco & $\mathrm{ABA}$ & 66 & 13 & 11 & $0.9615 \pm 0.0496$ & $2.615385 \pm 1.493860$ \\
\hline & Eleuthera & ELU & 60 & 13 & 11 & $0.9744 \pm 0.0389$ & $3.089744 \pm 1.715243$ \\
\hline & Exuma & EXU & 59 & 14 & 11 & $0.9341 \pm 0.0611$ & $3.406593 \pm 1.853374$ \\
\hline & Grand Bahama & GRB & 58 & 14 & 14 & $1.0000 \pm 0.0270$ & $3.472527 \pm 1.883809$ \\
\hline & Long Island & LIS & 43 & 1 & 1 & $1.0000 \pm 0.0000$ & N/A \\
\hline & New Providence & NWP & 142 & 25 & 23 & $0.9933 \pm 0.0134$ & $4.073333 \pm 2.101375$ \\
\hline \multirow{19}{*}{$\frac{\overleftrightarrow{c}}{\frac{\pi}{2}}$} & WEST & & & & & & \\
\hline & Angola & ANG & 230 & 46 & 26 & $0.9498 \pm 0.0181$ & $2.679227 \pm 1.452790$ \\
\hline & Benin & BEN & 78 & 20 & 16 & $0.9632 \pm 0.0328$ & $3.115789 \pm 1.686867$ \\
\hline & Burkina Faso & BRF & 335 & 13 & 12 & $0.9872 \pm 0.0354$ & $2.564103 \pm 1.469842$ \\
\hline & Cameroon (Bantu) & $\mathrm{CAB}$ & 28 & 13 & 9 & $0.9487 \pm 0.0423$ & $2.756410 \pm 1.559815$ \\
\hline & Cameroon (Pygmy) & $\mathrm{CPY}$ & 27 & 10 & 6 & $0.8889 \pm 0.0754$ & $1.888889 \pm 1.174734$ \\
\hline & Gabon & GAB & 828 & 302 & 130 & $0.9788 \pm 0.0037$ & $3.255484 \pm 1.682299$ \\
\hline & Ivory Coast & IVC & 90 & 13 & 9 & $0.9359 \pm 0.0507$ & $3.423077 \pm 1.870144$ \\
\hline & Nigeria & NIG & 12 & 8 & 7 & $0.9643 \pm 0.0772$ & $3.571429 \pm 2.026089$ \\
\hline & CENTRAL & & & & & & \\
\hline & Botswana & BOT & 40 & 10 & 6 & $0.9111 \pm 0.0620$ & $1.977778 \pm 1.217904$ \\
\hline & Central African Republic (Pygmies) & CRP & 23 & 10 & 7 & $0.9111 \pm 0.0773$ & $3.133333 \pm 1.771944$ \\
\hline & Democratic Republic of the Congo (Bantu) & DRC & 69 & 16 & 11 & $0.9500 \pm 0.0364$ & $2.308333 \pm 1.332841$ \\
\hline & Uganda (Nilotes) & UGA & 118 & 6 & 5 & $0.9333 \pm 0.1217$ & $2.800000 \pm 1.714643$ \\
\hline & Zambia (Bantu) & ZAM & 549 & 125 & 75 & $0.9818 \pm 0.0048$ & $3.095613 \pm 1.618909$ \\
\hline & EAST & & & & & & \\
\hline & Kenya (Nilotic) & KEN & 79 & 10 & 6 & $0.8444 \pm 0.1029$ & $3.111111 \pm 1.761373$ \\
\hline & Tanzania (Bantu) & $\mathrm{TAB}$ & 131 & 15 & 12 & $0.9429 \pm 0.0542$ & $2.771429 \pm 1.552708$ \\
\hline & Tanzania (Khoisan) & TAK & 135 & 19 & 10 & $0.9181 \pm 0.0364$ & $2.660819 \pm 1.483220$ \\
\hline
\end{tabular}

Supplementary Table 7: Haplotype Diversity indices for E1b1a7a-U174 individuals 


\begin{tabular}{|c|c|c|c|c|c|c|c|}
\hline & Population & Abbreviation & $\mathbf{N}$ & $\begin{array}{c}\text { Number of different } \\
\text { haplotypes }\end{array}$ & $\begin{array}{c}\text { Number of unique } \\
\text { haplotypes }\end{array}$ & $\begin{array}{c}\text { Haplotype } \\
\text { diversity (HD) }\end{array}$ & $\begin{array}{c}\text { Mean number of } \\
\text { pairwise differences }\end{array}$ \\
\hline \multirow{6}{*}{$\sum_{\infty}^{\infty}$} & Abaco & $\mathrm{ABA}$ & 66 & 17 & 13 & $0.9632 \pm 0.0328$ & $4.198529 \pm 2.193222$ \\
\hline & Eleuthera & ELU & 60 & 11 & 8 & $0.9455 \pm 0.0535$ & $2.272727 \pm 1.349494$ \\
\hline & Exuma & EXU & 59 & 11 & 9 & $0.9455 \pm 0.0659$ & $3.181818 \pm 1.780287$ \\
\hline & Grand Bahama & GRB & 58 & 11 & 11 & $1.0000 \pm 0.0388$ & $3.909091 \pm 2.122044$ \\
\hline & Long Island & LIS & 43 & 6 & 6 & $1.0000 \pm 0.0962$ & $2.800000 \pm 1.714643$ \\
\hline & New Providence & NWP & 142 & 28 & 21 & $0.9815 \pm 0.0133$ & $2.862434 \pm 1.552509$ \\
\hline \multirow{17}{*}{ 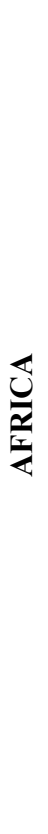 } & WEST & & & & & & \\
\hline & Angola & ANG & 230 & 143 & 65 & $0.9467 \pm 0.0099$ & $2.470009 \pm 1.342948$ \\
\hline & Benin & BEN & 78 & 7 & 6 & $0.9524 \pm 0.0955$ & $4.142857 \pm 2.342311$ \\
\hline & Burkina Faso & BRF & 335 & 86 & 51 & $0.9688 \pm 0.0089$ & $2.945554 \pm 1.557865$ \\
\hline & Cameroon (Bantu) & $\mathrm{CAB}$ & 28 & 6 & 5 & $0.9333 \pm 0.1217$ & $1.800000 \pm 1.200000$ \\
\hline & Gabon & GAB & 828 & 289 & 126 & $0.9764 \pm 0.0037$ & $3.088932 \pm 1.609946$ \\
\hline & Ivory Coast & IVC & 90 & 31 & 22 & $0.9656 \pm 0.0188$ & $3.053763 \pm 1.633555$ \\
\hline & CENTRAL & & & & & & \\
\hline & Botswana & BOT & 40 & 13 & 11 & $0.9744 \pm 0.0389$ & $3.115385 \pm 1.727175$ \\
\hline & Central African Republic (Pygmies) & CRP & 23 & 6 & 3 & $0.7333 \pm 0.1552$ & $3.466667 \pm 2.053723$ \\
\hline & Democratic Republic of the Congo (Bantu) & DRC & 69 & 22 & 17 & $0.9740 \pm 0.0217$ & $3.571429 \pm 1.885750$ \\
\hline & Uganda (Nilotes) & UGA & 118 & 7 & 5 & $0.9048 \pm 0.1033$ & $3.000000 \pm 1.776867$ \\
\hline & Zambia (Bantu) & ZAM & 549 & 309 & 113 & $0.9633 \pm 0.0058$ & $2.803282 \pm 1.484925$ \\
\hline & EAST & & & & & & \\
\hline & Kenya (Nilotic) & KEN & 79 & 6 & 4 & $0.8000 \pm 0.1721$ & $2.133333 \pm 1.372751$ \\
\hline & Tanzania (Bantu) & TAB & 131 & 13 & 11 & $0.9744 \pm 0.0389$ & $2.794872 \pm 1.577781$ \\
\hline & Tanzania (Khoisan) & TAK & 135 & 22 & 15 & $0.9524 \pm 0.0291$ & $3.060606 \pm 1.655276$ \\
\hline
\end{tabular}

\section{Supplementary Table 8: Haplotype Diversity indices for E1b1a8-U175 individuals}




\begin{tabular}{|c|c|c|c|c|c|c|c|}
\hline & Population & Abbreviation & $\mathbf{N}$ & $\begin{array}{c}\text { Number of different } \\
\text { haplotypes }\end{array}$ & $\begin{array}{c}\text { Number of unique } \\
\text { haplotypes }\end{array}$ & $\begin{array}{c}\text { Haplotype } \\
\text { diversity (HD) }\end{array}$ & $\begin{array}{c}\text { Mean number of } \\
\text { pairwise differences }\end{array}$ \\
\hline \multirow{6}{*}{$\sum_{\infty}^{\infty}$} & Abaco & $\mathrm{ABA}$ & 66 & 11 & 10 & $0.9818 \pm 0.0463$ & $3.781818 \pm 2.062357$ \\
\hline & Eleuthera & ELU & 60 & 6 & 6 & $1.0000 \pm 0.0962$ & $4.000000 \pm 2.323790$ \\
\hline & Exuma & EXU & 59 & 5 & 4 & $0.9000 \pm 0.1610$ & $2.800000 \pm 1.768560$ \\
\hline & Grand Bahama & GRB & 58 & 8 & 8 & $1.0000 \pm 0.0625$ & $2.392857 \pm 1.450362$ \\
\hline & Long Island & LIS & 43 & 24 & 14 & $0.9565 \pm 0.0204$ & $3.775362 \pm 1.971008$ \\
\hline & New Providence & NWP & 142 & 26 & 22 & $0.9846 \pm 0.0160$ & $3.326154 \pm 1.764516$ \\
\hline \multirow{11}{*}{ 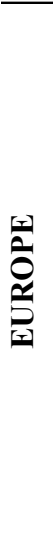 } & Greece (North) ${ }^{\dagger}$ & GRE & 191 & 19 & 19 & $1.0000 \pm 0.0171$ & $3.637427 \pm 1.927505$ \\
\hline & Italy & ITA & 155 & 57 & 40 & $0.9843 \pm 0.0070$ & $3.547619 \pm 1.830824$ \\
\hline & Ireland $^{\dagger}$ & IRE & 178 & 124 & 86 & $0.9891 \pm 0.0035$ & $3.301338 \pm 1.708942$ \\
\hline & Macedonia $^{\dagger}$ & MAC & 150 & 20 & 17 & $0.9789 \pm 0.0245$ & $3.389474 \pm 1.810919$ \\
\hline & Montenegro & MON & 404 & 38 & 14 & $0.8094 \pm 0.0596$ & $1.990043 \pm 1.148524$ \\
\hline & Poland $^{\dagger}$ & POL & 208 & 18 & 16 & $0.9804 \pm 0.0284$ & $3.549020 \pm 1.892296$ \\
\hline & Portugal $^{\dagger}$ & POR & 250 & 136 & 98 & $0.9818 \pm 0.0063$ & $3.488780 \pm 1.789699$ \\
\hline & Romania $^{\dagger}$ & ROM & 122 & 15 & 14 & $0.9905 \pm 0.0281$ & $4.685714 \pm 2.431234$ \\
\hline & Serbia & SER & 179 & 8 & 6 & $0.8929 \pm 0.1113$ & $1.607143 \pm 1.059816$ \\
\hline & Spain $^{\dagger}$ & SPN & 144 & 88 & 74 & $0.9919 \pm 0.0046$ & $3.730930 \pm 1.901473$ \\
\hline & United Kindgom (Caucasian) ${ }^{\dagger}$ & UKC & 250 & 165 & 118 & $0.9916 \pm 0.0025$ & $3.529120 \pm 1.805350$ \\
\hline
\end{tabular}

$\dagger$ Y-haplogroups were inferred from Y-STR haplotypes provided by the authors using Athey's Haplogroup Predictor 23-Haplogroup Beta program (Athey, 2006)

Supplementary Table 9: Haplotype Diversity indices for R1b1b1-M269 individuals 


\section{CONCLUSION}

The results of this investigation demonstrate that Afro-Caribbeans (i.e., Bahamians, Haitians and Jamaicans) are a heterogeneous group, sharing different proportions of their autosomal component with African, European, East Asian and, in the case of the Bahamian collections, Native American sources. This genetic variation, in combination with the presence of unique alleles in each island sampled, advocates the establishment of insular-specific databases for the assessment of profile frequencies and probability of inclusion as well as exclusion determinations. Interestingly, the substantial proportion of African DNA present in the gene pool of each Bahamian island was also observed when utilizing high-resolution Y-chromosome binary markers, with the collections from Abaco, Exuma, Eleuthera, Grand Bahama and New Providence receiving the strongest African genetic signals while the Long Island populace, in contrast, derives the majority of its paternal component from Europeans. Therefore, it is not surprising that African influences remain an important source of cultural traditions throughout the archipelago. Altogether, this data should provide a better understanding about the formation of the present-day Bahamian population, its current genetic makeup, and how different migratory events have shaped the genetic composition of each of the six islands sampled. 
VITA

TANYA M. SIMMS

Born

August 24, 1979, Nassau, Bahamas

EDUCATION

2011

Doctoral Candidate in Biology

Department of Biological Sciences

Florida International University

Miami, Florida

2005

Emergency Medical Technician Training Course

Florida Medical Training Institute, Inc.

Miami, Florida

2003

M.S., Biomedical Sciences

Barry University

Miami, Florida

2001

B.S., Biology

Barry University

Miami, Florida

1998

A.A., Biology/Chemistry

College of the Bahamas

Nassau, Bahamas

\section{PRESENTATIONS}

Genetic Heritage of Afro-Caribbean populations: A Tale Unearthed by Autosomal STRs. Tanya M. Simms*, Carol E. Rodriguez, Rosa Rodriguez, Rene J. Herrera. FIU Annual Biology Research Symposium. Florida International University. February 2010. Miami, FL.

\section{PUBLICATIONS}

Simms TM, Garcia C., Mirabal S, McCartney Q, Herrera RJ. (2008). The Genetic Legacy of the Transatlantic Slave Trade in the Island of New Providence. Forensic Science International Genetics 2:310-317. 
Gayden T, Mirabal S, Cadenas AM, Lacau H, Simms TM, Morlote D, Chennakrishnaiah S, Herrera RJ. (2009). Genetic Insights into the origins of Tibeto-Burman populations in the Himalayas. Journal of Human Genetics 54:216-223.

Simms TM, Rodriguez CE, Rodriguez R, Herrera RJ. (2010). The Genetic Structure of Populations from Haiti and Jamaica Reflect Divergent Demographic Histories. American Journal of Physical Anthropology 142:49-66.

Tanya M. Simms*, Anna Goldina*, Thomas Pitzer. (2010). General Biology I Laboratory Manual. New York, NY: McGraw-Hill.

Anna Goldina*, Tanya M. Simms*, Thomas Pitzer. (2010). General Biology II Laboratory Manual. New York, NY: McGraw-Hill.

Regueiro M*, Stanojevic A*, Chennakrishnaiah S, Rivera L, Varljen T, Alempikevic D, Stojkovic O, Simms T, Gayden T, Herrera RJ. (2011). Divergent Patrilineal Signals in three Roma Populations. American Journal of Physical Anthropology 144:80-91.

Simms TM, Barrett DA, McCartney Q, Herrera RJ. Divergent Genetic Strata in five Bahamian Islands. In Press Forensic Science International: Genetics.

Simms TM, Martinez E, Herrera KJ, Wright MR, Perez OA, Hernandez $\mathrm{M}^{\dagger}$, Ramirez $\mathrm{EC}^{\dagger}$, McCartney Q, Herrera RJ. The Patriarchal Genetic Substrata of the Bahamian Archipelago. In Preparation American Journal of Physical Anthropology.

\section{AWARDS AND CERTIFICATIONS}

- FIU Dissertation Year Fellowship (2010-2011)

- Graduate Teaching Assistants Excellence-In-Teaching Award (2008)

- Emergency Medical Technician - Basic (2005-2007) 